Journal of

Nutrigenetics

Nutrigenomics

\title{
4th Congress of the International Society of Nutrigenetics/Nutrigenomics (ISNN)
}

\section{Abstracts}

November 18-20, 2010,

Pamplona, Spain
Guest Editors

Raffaele De Caterina, Chieti/Pisa

J. Alfredo Martínez, Pamplona 
Journal of

Nutriga of
and
Nutrigenomics

President

Raffaele De Caterina (Chieti, Italy)

Past President

Artemis P. Simopoulos (Washington, D.C, USA)

Secretary / Treasurer

Jing X. Kang (Charlestown, USA)

\section{Members}

Michel De Lorgeril (Grenoble, France)

Lynnette R. Ferguson (Auckland, USA)

Serge Ferrari (Geneva, Switzerland)

Ronald Krauss (Oakland, USA)

Renato Mariani-Constantini (Chieti, Italy)

Alfredo Martínez (Pamplona, Spain)

John A. Milner (Rockville, USA)

Sergio Muntoni (Cagliary, Italy)

Marjanne Senekal (Cape Town, South África)

Charles N. Serhan (Boston, USA)

Gérard Siest (Nancy, France)

Antonio Velázquez (México City, México)

Nikos Yiannakouris (Athens, Greece) 


\section{Journal of \\ Nutrigenetics \\ Nutrigenomics}

Dear Colleagues,

On behalf of the Board of directors of ISNN the Scientific Planning Committee and members of the University of Navarra Organizing Committees, it is our pleasure to invite you to this 4th Congress of the International Society of Nutrigenetics/ Nutrigenomics (ISNN) "From reference intakes to personalized nutrition" held at the University of Navarra, Pamplona (Navarra), Spain, in November 18-20, 2010.

The Congress is designed to provide a comprehensive overview of the latest research and translational developments in Nutrigenetics and Nutrigenomics. This rapidly moving field in personalized nutrition overlaps with all aspects of health and/ or disease, including normal growth, development, obesity, cardiovascular disease, diabetes, and cancer. It is also focused on fundamental interrelationships between food components and key cellular processes including inflammation, hormonal regulation, and cell division/differentiation. Indeed, nutrigenetics, nutrigenomics and epigenomics issues represent the future for both primary and secondary disease prevention and treatment, which will be dealt with in this IV ISNN Congress.

We would like to express our sincere appreciation to national and regional government agencies and to manufacturing industries (food, pharmaceutical and technological companies), for their generous support that is making this Congress possible. We also wish to express special thanks to Iberia Viajes for their excellent arrangements in all aspects of the Congress organization, and to our dedicated staff, colleagues, and friends for their untiring help, support and advice in planning and arranging this meeting.

It is also worth mentioning that we are very grateful to the editorial team of the Journal of Nutrigenetics and Nutrigenomics for their precise work, concerning the abstracts of the meeting, and to Patrick Naef and Dr. González-Muniesa for editorial assistance.

We hope that the Congress fosters a creative exchange of ideas and builds/enhances interactions with colleagues from many different countries. We are convinced it will be a rewarding experience for all. Thank you for joining us at this very beautiful and exciting city of Pamplona in November 2010, the town where bulls run freely in the streets five minutes a day during a week in the summer season.

Warmest Regards
J. Alfredo Martínez Congress Chairman

Raffaele De Caterina ISNN President

\begin{tabular}{ll}
\hline KARGER & (c) 2010 S. Karger AG, Basel \\
Fax +4161306 1234 $361-6499 / 10 / 0033-0049 \$ 26.00 / 0$ \\
$\begin{array}{l}\text { E-Mail karger@karger.ch } \\
\text { www.karger.com }\end{array}$ & $\begin{array}{l}\text { Accessible online at: } \\
\text { www.karger.com/jnn }\end{array}$
\end{tabular}




\section{Program}

\section{Journal of \\ Nutrigenetics \\ Nutrigenomics}

\section{Wednesday, November 17}

10:00 $\quad 7^{\text {th }}$ CTP Symposium (1 ${ }^{\text {st }}$ part) at University of Navarra

11:30 Coffee break (Science Library Lecture Room)

12:30 Catedra Pascual Symposium (Science Library Lecture Room at University of Navarra)

Round table about the future of nutrigenomics: Studies in USA and Spain.

J. Alfredo Martínez, University of Navarra, Spain

Marta Garaulet, University of Murcia, Spain

Dolores Corella, University of Valencia, Spain

14:00 Lunch

15:30 $\quad 7^{\text {th }}$ CTP Symposium ( $2^{\text {nd }}$ part $)$

16:45 Coffee break

17:00 LECO, Metabolomics in Nutrition Research

17:30 TSE, Novel Technologies in Automated Animal Testing and Phenotyping

18:00 Tebu Bio, Gene and Protein Expression Assessment in Nutrition and Obesity

\begin{tabular}{ll}
\hline & IV ISNN CONGRESS WELCOME \\
\hline $18: 45$ & Welcome at the University of Navarra, Central Building \\
19:00 & $\begin{array}{l}\text { Special Lecture. VI Edition of the Nutrition and Health Award, University of Navarra } \\
\text { José M. Ordovás, Tufts University, Boston, USA, and Spanish National Cardiovascular Research Center (CNIC), Spain }\end{array}$ \\
20:00 & Cocktail at Central Building, University of Navarra
\end{tabular}

Thursday, November 18

08:00 Registration at Baluarte Conference Center

08:30 Congress Opening

Welcome address by the ISNN President Raffaele De Caterina and the Chairman of the IV Congress of

Nutrigenomics and Nutrigenetics J. Alfredo Martínez

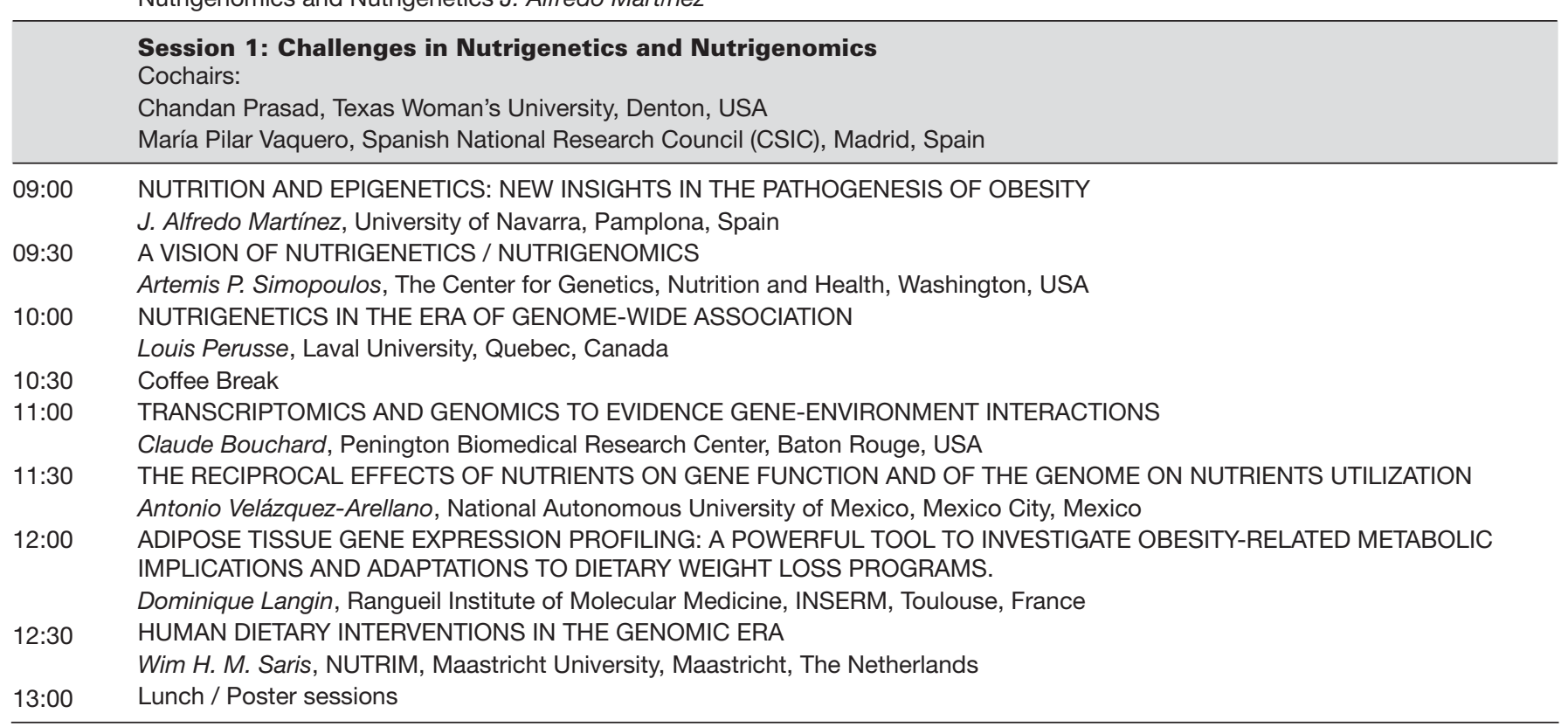

\section{KARGER}

Fax +41613061234

E-Mail karger@karger.ch

www.karger.com
(C) 2010 S. Karger AG, Basel

Accessible online at:

www.karger.com/jnn 


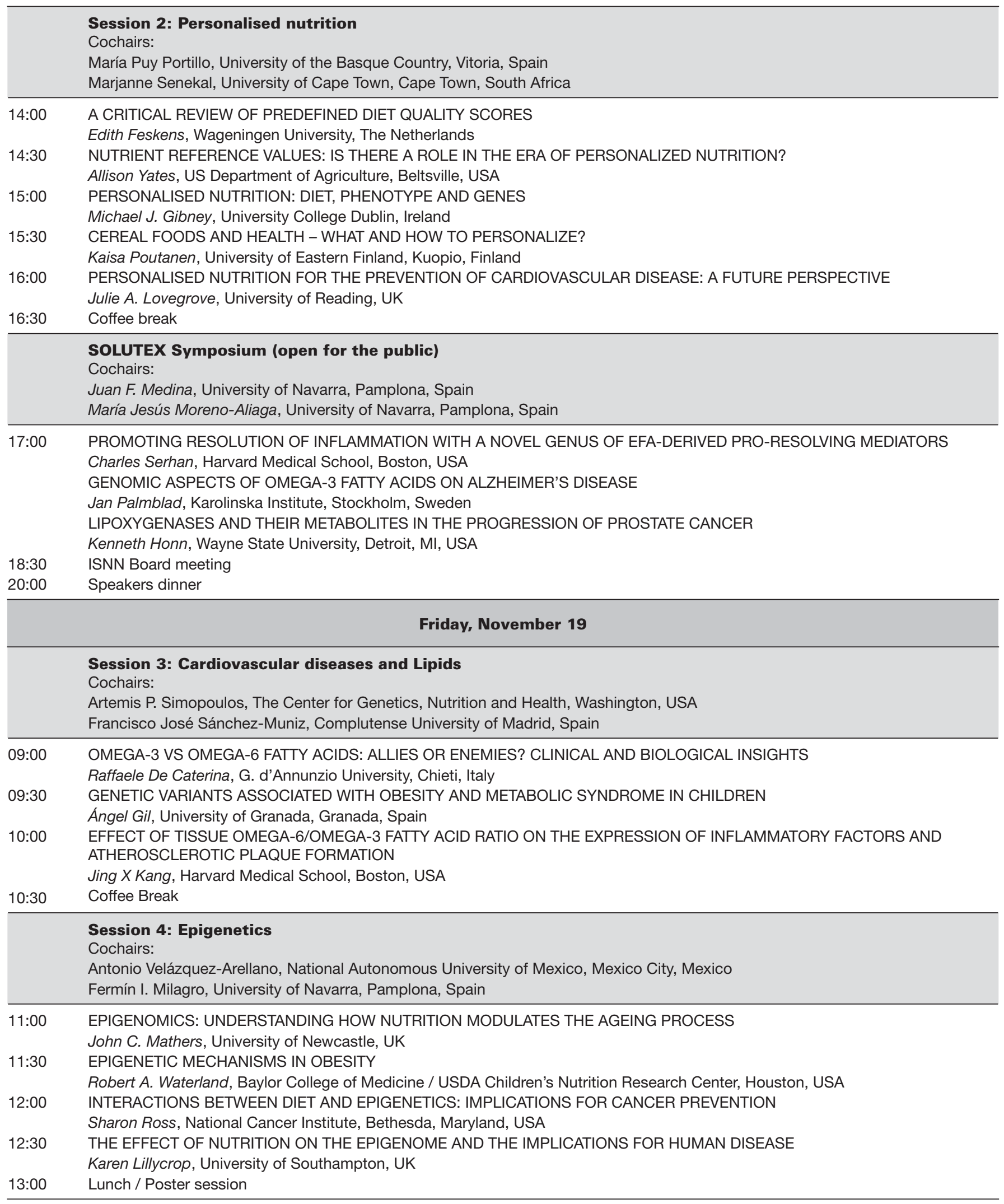




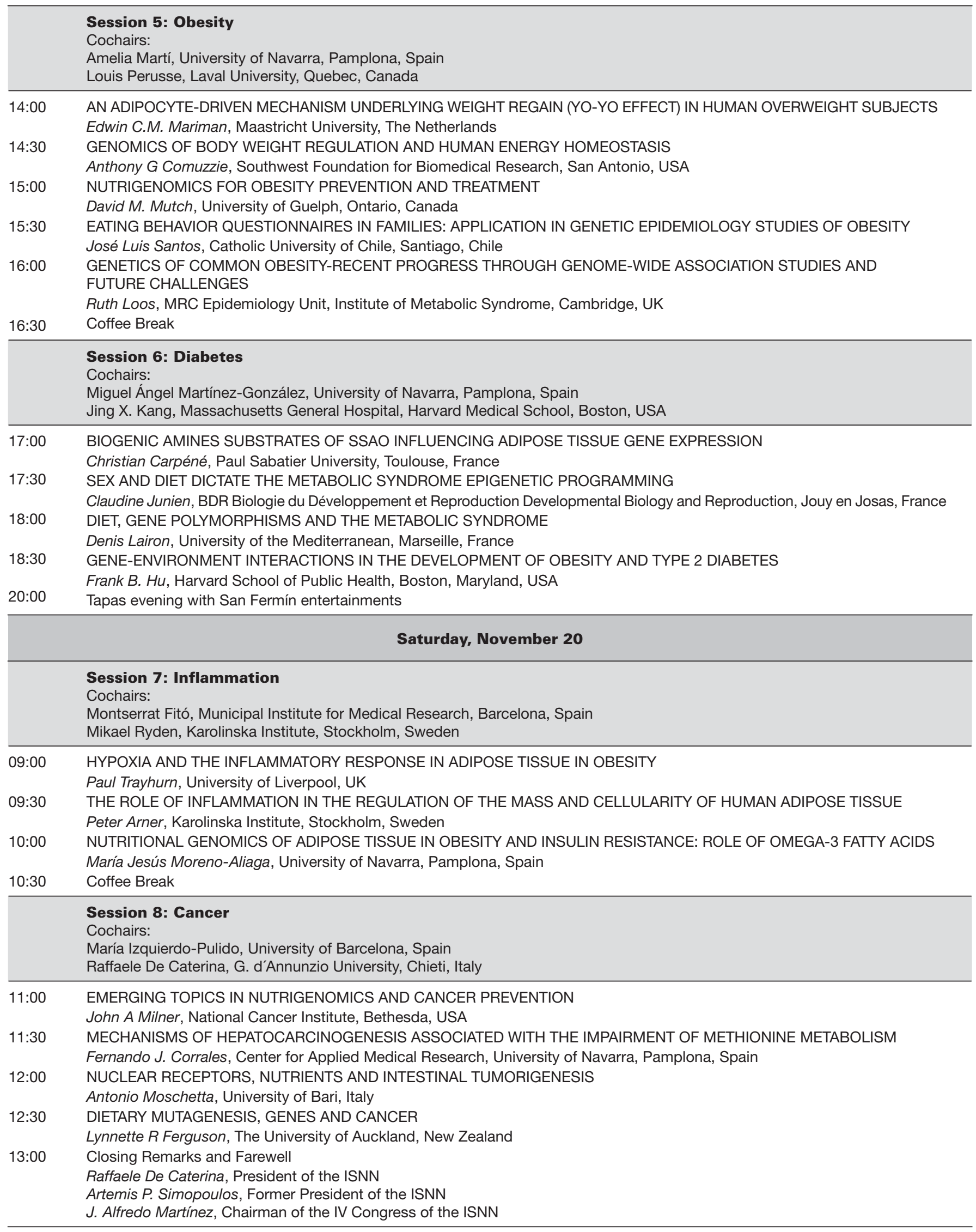




\section{Journal of \\ Nutrigenetics \\ Nutrigenomics}

\section{Challenges}

01

\section{Nutrition and Epigenetics: New Insights in the Pathogenesis of Obesity}

\section{J.A. Martinez, J. Campion, F.I. Milagro}

Institute of Nutrition and Food Sciences, University of Navarra, Pamplona, Spain

Obesity is the result of an interaction between the dietary factors and lifestyles with the genetic make-up. Nowadays, epigenetics is emerging as a link that may explain the role of early life exposure to nutrients, stress and maternal care on the risk of developing obesity and metabolic diseases in adulthood.

The most studied epigenetic regulatory mechanisms are DNA methylation patterns in cytosines next to guanines ( $\mathrm{CpG}$ sites) and covalent modifications on the terminal amino acid tails of histones, involving methylation, acetylation ubiquitination and other reactions. A number of dietary compounds, such as polyphenols, butyrate, amino acids, minerals or vitamins, or different processes related to obesity, including aging, hypoxia, inflammation, adipocyte hypertrophy or hyperglycemia, can modify histone acetylation and methylation, contributing to epigenetically regulate the transcriptome.

Regarding DNA methylation, the expression of different genes playing a role in the onset of obesity and related processes, termed as epiobesogenes, could be controlled by cytosine methylation. Some of these genes are involved in inflammation, such as TNF-alpha or CD44, in appetite regulation, such as leptin, in lipid trafficking, such as ATP10A, in glycerol transport, such as aquaporin 9, or in energy metabolism and cancer, such as WT1. Some of these epiobesogenes are epigenetically regulated by a high fat intake or by weight loss. Such epigenetic "marks" could be used to predict the outcome of weight loss programs or to determine the susceptibility to suffer obesity, type 2 diabetes, cardiovascular events or cancer later in life. Moreover, the interindividual variability of the epigenetic background seems to play a role in the success of weight management programs.

Future advances in omics technologies will integrate the epigenetic, genetic and nutritional knowledge in the development of personalized nutrition and clinical care. The identification at an early age of individuals that present specific methylation profiles in recognized genes would also allow the prevention and monitoring of their evolution, whereas dietary supplementation could be used as a mean of counteracting adverse epigenomic profiles in an individualized-tailored manner.

\section{2}

\section{A Vision of Nutrigenetics/Nutrigenomics}

\section{Artemis P. Simopoulos}

The Center for Genetics, Nutrition and Health, Washington, D.C., USA

Our understanding of the genotypic contribution to determining nutritional requirements is in the process of being revolutionized by molecular biology and studies on Nutrigenetics/Nutrigenomics. Studies on genetic variants and their response to diet in health and disease (Nutrigenetics) as well as studies on the role of nutrients in gene expression (Nutrigenomics) lead the way in the development of personalized nutrition and in the definition of a healthy diet and development of Novel Foods. To continue in its role as an integrator of genomic and environmental processes, Nutritional science is adjusting its focus to include the microstructure of the genome, the metabolome, the proteome, the epigenome, etc. Our success in this regard will be reflected by our ultimate shedding of the statistical trappings required by the study of systems with high variance, ultimately we will be able to understand nutritional metabolism at levels of discrimination sufficient to permit individual dietary prescription. Genetic variation bears on the development of public health policy as well as on the delivery of healthcare at the individual level. When nutritional recommendations are made, genetic variability among individuals in the population will need to be taken into consideration. If the number of individuals affected by the genetic variation becomes important for policy setting, their benefit must be considered from the standpoint of both society and the individual. As we advance our knowledge of gene nutrient interactions, society will need to create or utilize appropriate social, ethical, legal, educational, economic, agricultural and industrial (i.e. development of novel foods) frameworks to gain the benefits of such knowledge. Public health and regulatory processes will need to be established to define when genomic discoveries such as gene/nutrient disease associations are ready to be evaluated as potential tools to improve health screening and recommended dietary values.

\section{KARGER \\ (C) 2010 S. Karger AG, Basel}

Fax +4161306 1234

E-Mail karger@karger.ch

www.karger.com www.karger.com/jnn
Accessible online at: 


\section{3}

\section{Nutrigenetics in The Era of Genome-Wide Association Studies}

\section{Louis Pérusse}

Department of Preventive Medecine and Institute of Nutraceutical and Functional Foods, Laval University, Quebec, Canada

Genome-wide association studies (GWAS) have identified a large number of genes and/or loci associated with common diseases and/or quantitative traits considered as risk factors for these diseases. Although GWAS clearly emerged as a powerful approach to identify common susceptibility variants, the effects of the variants identified so far were generally smaller than expected and explained only a small fraction of the heritability of the disease traits. The presence of gene-environment $(\mathrm{GxE})$ interactions is considered as one of the factors that could account for the missing heritability in GWAS. Most of the GWAS performed to date have focused on main genetic effects and have not fully explored the potential role environmental factors could play in modifying genetic risk. The objective of this presentation is to demonstrate how data from genome-wide association studies can be used to investigate gene-nutrient interactions. Results of a GWAS for obesity and dietary intakes performed in the Quebec Family Study will be first reviewed. We will then examine whether the obesity genes identified through GWAS have an impact on dietary intakes. We will also provide examples showing that modeling of dietary factors in GWAS can lead to the discovery of novel genes.

\section{4 \\ Transcriptomics and Genomics to Evidence Gene-Environment Interactions}

\section{Claude Bouchard}

Human Genomics Laboratory, Pennington Biomedical

Research Center, Baton Rouge, La., USA

Rationale: We propose that one of the most powerful ways to investigate gene-environment interaction effects and resolve them in terms of genes and DNA sequence variants is to use transcriptomics and genomics technologies in completers of well defined experimental studies in which the environmental exposure if fully standardized.

Methods: This approach will be illustrated by a series of experiments designed to identify the genes and single nucleotide polymorphisms (SNPs) contributing to variation in the changes brought about by exercise training in cardiorespiratory fitness as assessed by maximal oxygen uptake $\left(\mathrm{VO}_{2} \max \right)$ and insulin sensitivity.

Results: In the HERITAGE Family Study, among 742 sedentary adults, the response of $\mathrm{VO}_{2}$ max to standardized exercise training, adjusted for baseline level, age, sex and ethnicity is influenced by a genetic component of the order of $50 \%$, suggesting that the genotypeexercise interaction effect is strong. The heterogeneity of responses to training is considerable, with a range from about fourfold to tenfold when comparing the lowest to the highest responders. A similar range is observed for changes in fasting insulin and insulin sensitivity but the genetic component reaches only about $25 \%$. Transcriptomics from skeletal muscle samples yielded panels of transcripts that predicted exercise training-induced changes. In the case of the gains in $\mathrm{VO}_{2}$ max, a panel of 29 transcripts reflecting the baseline level of transcription accounted for $58 \%$ of the variance. SNPs were typed in the genes encoding these transcripts and in 10 other candidates generated through other approaches. SNPs in 11 of these genes were shown to account for $23 \%$ of the variance in $\mathrm{VO}_{2}$ max training response in HERITAGE. Likewise several muscle transcripts were associated with the exercise-induced insulin metabolism changes. In particular, FHL1 transcript abundance was associated with the changes in insulin and glucose metabolism and SNPs in the same gene correlated with the magnitude of the improvements.

Implications: Combining transcriptomics and genomics to define the architecture of gene-nutrient interactions effects would be valuable provided that high levels of compliance with the prescribed dietary interventions could be achieved.

\section{5 \\ The Reciprocal Effects of Nutrients on Gene Function, and of The Genome on Nutrients} Utilization

Antonio Velázquez-Arellano

Unidad de Genética de la Nutrición, Instituto de Investigaciones Biomédicas, Universidad Nacional Autónoma de Mexico e Instituto Nacional de Pediatría, Mexico City, Mexico

Organisms and their cells perceive the constantly changing availability of energy and nutrients, and continuously modify their genome functioning accordingly. Cellular responses include changes in multiple processes, signaling pathways and gene expression patterns, in turn modifying amounts and locations of regulatory proteins. This reshaping of genome operation thus modulates metabolic processes, leading to adaptations that optimize the organisms' nutrition.

Impairment of any step in the complex circuitries underlying these processes leads to disease. It is now known that most cases of all common chronic disorders result from combinations of altered components of these circuitries, their causation thus being multifactorial and heterogeneous, steered by interactions among polygenic mutations, abnormal nutrient intakes and unfit life styles.

Efforts to identify components of the complex responses to dietary signals have become increasingly successful by the advent of new approaches and techniques, many of which produce high-throughput data being interpreted by sophisticated data mining tools. All this is rapidly changing our views of nutrition in health and disease.

This presentation will give a succinct overview of current understanding of some of the mechanisms that mediate genomic adaptations to nutrient intake, exemplified by glucose and the vitamin biotin.

Direct glucose sensing allows free-living unicellular organisms, such as the baker's yeast Saccharomyces cerevisiae, to live in a constantly changing nutrient environment. With multicellularity, an "internal milieu" and the appearance of a circulatory system, humans and the other higher animals have evolved immensely more complex regulatory circuitries comprising hormonal and neural signals, signal transduction pathways, transcriptional and translational components 
and epigenetic mechanisms. However, the basic logic and components of these circuitries have largely been conserved along one thousand million years of eukaryotes evolution.

This multiplicity of regulatory pathways and their components and control processes has been paralleled with an equally large increase of the non-coding part of the genomes, and has multiplied the vulnerable targets whose changes augment the risks for energy management diseases such as diabetes, atherosclerosis and cancer. But this is also why, despite the impressive strides in the "omics" disciplines, their practical applications have thus far been meager. With breakthroughs like the new sequencing technologies this situation may change in the near and midterm future.

(This work was partly supported by research grants from DGAPA/PAPIIT (IN225508-3) of the Universidad Nacional Autónoma de México, and from Consejo Nacional de Ciencia y Tecnología (57597))

\section{6 \\ Human Dietary Interventions Studies in The Genomic Era}

Wim H.M. Saris

Nutrition Research institute NUTRIM, Maastricht

University, Maastricht, The Netherlands

The availability of the genomics technologies such as genetics, transcriptomics, proteomics and metabolomics has revolutionized Life Sciences. For the Nutritional Sciences the results and outcomes are less clear and certainly not yet revolutionized the nutritional sciences. Dietary intervention studies are mostly focused on health outcomes and accompanied claims. Therefore endpoints of nutritional interventions are mostly focussed on physiological functions and disease related biomarkers such as Flow-Mediated Dilatation. So far in dietary intervention studies the link between genomics technologies and endpoint measurements is relatively weak and should be strengthen. With the overwhelming amount of data coming out of these new technologies one should be able to predict more precisely earlier in the dietary intervention process whether molecular/cellular/ tissue effects will modify the targeted function or disease risk at an endpoint level later in the process. To achieve this goal these new technologies should be included in study protocols in order to accelerate the process of integration. This will lead to replacement in the future. Since we are dealing in dietary interventions with mostly subtle effects compare to pharmacological treatment the possibilities to get a better insight into the metabolic effects by pathway analysis will increase enormously.

A potential complete new field could be the use of these technologies in particular metabolomics to control for dietary compliance. So far the nutritional sciences are serious hampered due to the fact that food intake measures are notorious unreliable. Using urinary/blood metabolic profiles could possibly increase the control on dietary compliance.
07

Adipose Tissue Gene Expression Profiling: A Powerful Tool to Investigate Obesity-Related Metabolic Complications and Adaptations to Dietary Weight Loss Programs

Dominique Langin

Obesity Research Laboratory, Rangueil Institute of Molecular Medicine, Inserm, U858, UPS, University of Toulouse; CHU de Toulouse, Biochemistry Laboratory, Biology Institute of Purpan, Toulouse, France and FrancoCzech Laboratory for Clinical Research on Obesity, Third Faculty of Medicine, Prague, Czech Republic and Inserm, Toulouse, France

The identification of physiological and biological factors underlying the metabolic disturbances observed in obesity is a key step in developing better diagnostic tools and therapeutic strategies. Obesity is a complex phenomenon characterized by an increased fat mass in different anatomical sites. Despite years of investigation, it is not understood why visceral fat is more harmful than subcutaneous fat. The two depots show biological differences but it has not been demonstrated that these differences explain the development of obesity complications. In addition, the adaptations to weight loss, weight maintenance and nutrient contents of the diet are probably associated with different molecular mechanisms within adipose tissue. The use of DNA microarrays and related "omics" techniques that allow the comparison of global expression changes in thousands of genes between different conditions appears as a useful tool to identify the molecular determinants of obesity-related metabolic complications. Recent studies from our laboratory show an opposite regulation between metabolism and immune response genes including novel human adipose tissue-specific macrophage markers suggesting that impairment of fat cell metabolism favors an immune response in adipose tissue. Gene expression profiles from subcutaneous fat appear as discriminating as those from visceral fat with respect to obesity and metabolic complications. Moreover, subcutaneous adipose tissue macrophages and adipocytes show distinct patterns of gene regulation and association with insulin sensitivity in subcutaneous fat during the various phases of a dietary weight loss program. Differences in adipose tissue gene expression patterns between subjects successful and unsuccessful in maintaining weight are mainly due to weight variations rather than to differences in diet macronutrient composition. Within the frame of the European programs MolPage and Diogenes, we aim at improving the workflow from human adipose tissue sampling to mRNA biomarker quantitation using novel high throughput quantitative PCR technology (e.g., Fluidigm ${ }^{\circledR}$ technology). Expression of more than 250 genes selected as putative markers of obesity complications and dietary interventions were quantified on adipose tissue samples from more than 500 sujects before the dietary intervention, at the end of a caloric restriction phase and at the end of the following weight maintenance phase (i.e., $\sim 450,000$ qPCR determinations). The first results of the analysis will be presented. 


\section{Personalized Nutrition}

08

\section{Nutrient Reference Values: Is There a Role in The Era of Personalized Nutrition?}

\section{Allison A. Yates}

Beltsville Human Nutrition Research Center, ARS/USDA, USA

For at least a century we have known that individuals respond differently to their environment with respect to developing chronic disease - some of the variation in response is due to genetics and some is due to changes in environmental stimuli, of which diet is a major component. The science of epidemiology has evolved to identify associations between environmental differences and risk of disease in populations groups; from this basis, dietary recommendations for groups of individuals of whom little is known about specific risk factors have been developed. In terms of risk to the individual, we have new and better tools that allow us to identify and follow genetic profiles and environmental exposure and their contribution to risk. Varying responses to dietary factors are derived from a combination of these genetic differences as well as environmental effects. A key component of such research is the identification of biomarkers that are both specific for the disease or function and selective enough to be causally related to the specific nutrient or food. Most dietary guidelines currently provided, such as nutrient reference values, serve as benchmarks to ensure an adequate intake or to provide for optimal health. In the past they have been crafted based on comparatively few studies with even fewer subjects, who usually are diverse in age, background, genetics, and previous environmental exposure. Thus while they are useful when applied to groups in terms of risk of disease outcomes, they typically do not apply to all who fit the category for which they are developed. The era of personalized nutrition aims to identify individual risk, and thus provide personalized recommendations. The real challenge will be to use individual risk information to craft scientifically based dietary guidance for groups which include individuals of whom little is known about their individual risk of disease.

\section{9}

\section{Personalised Nutrition: Diet, Phenotype and Genes}

\section{Michael J. Gibney \\ UCD Institute of Food \& Health, University College Dublin, Belfield, Dublin, Ireland}

The concept of personalised nutrition has always been related to details of an individual's genetic code and the many enterprises that set out to deliver a service in this area, have failed. Individual SNPs may allow researchers understand diet and gene interactions in metabolism but given the very large number of SNPs, definitive dietary advice based on single SNPs is not possible. The unknown effect of nutrients on SNPs, other that those being studied, raise concerns over the ethics of such advice. In recent times, there has been a growth in interest in the pursuit of personalised nutrition, not based on genetic data but based on phenotypic and dietary data. Many services in the public and private sectors offer the opportunity to submit details of one's habitual diet and receive back details of one's pattern of nutrient intake together with advice on where the balance of nutrients needs to be improved. In some instances, advice on improved food choice can be offered and it would not be difficult to envisage in the future that such data could be related to the information technology services of supermarkets. A second rapidly emerging area is the provision of personalised data on phenotype. This can be in the form of biochemical data, which would also advise the individual on their personal nutrient balance and ultimately their food choice. It can also be in the form of biofeedback information on functional aspects of health. Home based measures of blood pressure are an existing example but this will be extended to many other physiological and indeed psychological measures all of which will again advise individuals on their personalised nutrition choices. Finally, whereas personalised nutrition is presently seen as operating at the level of the individual, there exists the possibility that dietary advice could be tailored to clusters of individuals who share a common metabolic profile or metabotype. A forthcoming $€ 9 \mathrm{~m}$ funded EU FP\& project on personalised nutrition will help inform us of future developments in this area.

\section{0 \\ Cereal Foods and Health - What and How to Personalize? \\ K. Poutanen \\ VTT Technical Research Centre of Finland, Espoo, Finland, and University of Eastern Finland, Department of Public Health and Clinical Nutrition, Kuopio, Finland}

Cereal foods are among the staple foods, providing around one third of our energy intake. Most of them are consumed as refined food, consisting of the starchy endosperm (white flour). Epidemiological evidence points out the importance of the outermost grain layers (bran), and the intake of foods containing bran, whole grain and cereal fibre have been shown to protect against chronic disease, such as cardiovascular disease and type 2 diabetes. In spite of sound evidence, leading to dietary recommendations to eat more whole grain foods, the mechanisms and the protective factors still remain at hypothesis level.

Our postprandial and long human intervention studies have indicated positive effects of rye bread on insulin metabolism ( Juntunen et al 2002, Laaksonen et al. 2005). The systems biology approach has both at transcriptomic and metabolomic levels brought new evidence about the mechanisms underlying these changes (Kallio et al 2007, Lankinen et al 2010). The current interest includes metabolomic analysis of rye metabolites in plasma and urine, and in metabolomic profiling at levels of foods, intestinal in vitro metabolites and in vitro cell cultures in order to identify the biologically active phytochemicals and cellular pathways supporting healthy glucose metabolism. 
In general, the development of applications of nutritional systems biology started with genotyping, the interest being extended to phenotyping, nutrityping, or metabotyping - finding individual differences in our diet-related risk factors and/or responses to diets, in order to provide diagnosis tools, more individual dietary counseling or specific foods targeted at a diverse consumer groups. Personalized nutrition aims to provide foods and diets according to individual needs. In the very near future, nutrition may be improved also by developing foods and tools motivating and assisting people in following an "average" healthy diet according to the dietary recommendations - but encouraging them to do that according to their personal sensory and consumer preferences.

Juntunen, K., Niskanen, L., Liukkonen, K., Poutanen, K., Holst, J. \& Mykkänen, H. (2002) Postprandial glucose, insulin, and incretin responses to grain products in healthy subjects. Am. J. Clin. Nutr. 75:254-262.

Laaksonen, D.E., Toppinen, L.K., Juntunen, K.S., Autio, K., Poutanen, K.S., Niskanen, L.K. and Mykkänen, H.M. (2005) Dietary carbohydrate modification enhances insulin secretion in individuals with the metabolic syndrome. Am. J. Clin Nutr. 82:1218-1227.

Kallio, P., Kolehmainen, M., Laaksonen, D.E., Kekäläinen, J., Salopuro, T., Sivenius, K.,Pulkkinen, L., Mykkänen, H., Niskanen, L.K., Uusitupa, M.I. and Poutanen, K.S. (2007), Dietary carbohydrate modification induces marked alterations in gene expression of white adipose tissue in persons with metabolic syndrome -the FUNGENUT Study. Am J. Clin Nutr 85:1417-1427.

Lankinen, M., Schwab, U., Gopalacharyulu, P.V., Seppälä-Laakso, T., Yetukuri, L., Sysi-Aho, M., Kallio, P., Suortti, T., Laaksonen, D.E., Gylling, H., Poutanen, K., Kolehmainen, M. and Oresic, M. (2010) Dietary carbohydrate modification alters serum metabolic profiles in individuals with the metabolic syndrome. Nutr. Metab. Cardiovasc. Dis. 249-257.

\section{1 \\ Personalised Nutrition for The Prevention of Cardiovascular Disease: A Future Perspective}

\section{Julie Lovegrove \\ Institute for Cardiovascular and Metabolic Research (ICMR), Department of Food and Nutritional Sciences, University of Reading, Reading, UK}

Cardiovascular disease (CVD) is responsible for significant morbidity and mortality in the Western and developing world. This multifactorial disease is influenced by many environmental and genetic factors. At present, public health advice involves prescribed population based recommendations, which have been largely unsuccessful in reducing CVD risk. This is due, in part, to individual variability in response to dietary manipulations that arises from nutrient-gene interactions. The shift towards personalised nutritional advice is a very attractive proposition, where, in principle, an individual can be given dietary guidance specifically tailored to their genotype. In this first post-genome decade it would seem reasonable to assume personalised nutrition therapy was the norm. However, the evidence-base for the impact of interactions between nutrients and fixed genetic variants on biomarkers of CVD risk is still relatively limited. This presentation will review the evidence for interactions between dietary fat and common single nucleotide polymorphisms (SNP) in genes associated with CVD risk including the apolipoprotein E (apoE) epsilon missense mutation. The emerging data on the impact of saturated fatty acid and long chain n-3 polyunsaturated fatty acid, particularly docosahexaenoic acid (DHA) intake, and apoE polymorphisms on a number of CVD risk markers will be addressed. An increased understanding of how this and other gene polymorphisms influence responsiveness to nutrients should facilitate the progression of personalised nutrition. However the ethical and social issues surrounding its routine use requires careful consideration. Currently there is rapid commercialization of personal genomic tests, yet it is of considerable concern that these are based on limited studies of clinical validity and few on health benefits and outcomes. Therefore it seems plausible that global application of personalised nutrition therapy may not be realised in the near future.

\section{Cardiovascular Diseases}

\section{2 \\ Omega-3 vs Omega-6 Fatty Acids: Allies or Enemies? Clinical and Biological Insights}

\author{
Raffaele De Caterina \\ President, International Society of Nutrigenetics and \\ Nutrigenomics, Chair of Cardiology, "G. d'Annunzio" \\ University, Chieti, Italy
}

Human beings evolved on a diet with a ratio of omega- 6 to omega-3 fatty acids of approximately 1 , whereas in Western diets the ratio is $15 / 1$ or more. Western diets are deficient in omega- 3 fatty acids, and have high amounts of omega- 6 fatty acids compared with the diet on which human beings evolved and their genetic patterns were established. It has been stated therefore that excessive amounts of omega- 6 fatty acids and a very high omega-6/omega-3 ratio, as is found in today's Western diets, may promote the pathogenesis of many diseases, including cardiovascular disease, cancer, and inflammatory and autoimmune diseases, whereas increased absolute - but also relative - levels of omega-3 PUFA (a lower omega-6/omega-3 ratio), exert suppressive effects. Conversely, other investigators have postulated a greater relevance of the sole omega- 3 fatty acids intake, estimated by the simple measurement of EPA and DHA in erythrocyte membranes as a percentage of total erythrocyte fatty acids (the "omega-3 index"). This issue is still at the moment controversial. Although epidemiological studies in Western populations, in addition to showing an inverse relationship of cardiovascular morbidity and mortality with the estimated dietary intake of omega-3 fatty acids, have also shown in general a similar, albeit overall weaker, inverse relationship with the intake of omega- 6 fatty acids, most such evidence comes from studies where dietary PUFA (mostly omega-6) intake substituted saturated fatty acids. No randomized controlled trial has really independently tested the hypothesis of beneficial changes of selective changes of the ratio. On the other hand, several 
differences in study outcomes with omega- 3 fatty acids in different populations can be explained by the different background intake with the diet. While controlled increased intake of omega-3 fatty acids reduces the risk of sudden death in Western populations, it apparently does not so in Japanese, who have a much higher dietary intake of fish, consistent with an interaction with the background diet and a relevance of the ratio.

\section{3 \\ Genetic Variants Associated with Obesity and Metabolic Syndrome in Children}

Angel Gil

Department of Biochemistry and Molecular Biology II, Institute of Nutrition and Food Technology, Centre for

Biomedical Research, University of Granada, Spain

Background: The worldwide increasing obesity prevalence in childhood is associated with a higher prevalence of cardiovascular and metabolic diseases. Obesity has been linked to the metabolic syndrome (MS) or insulin resistance syndrome (IRS). Over 600 genes, markers and chromosomal regions have been associated with obesity and recent wide-genome analysis (WGA) has identified new loci and genes related to both obesity and MS. However, only a few of them have been specifically associated with obesity and MS in childhood.

Objectives: To discuss the role that the recent surge in findings from genome-wide association studies (GWASs) and case-control studies may play in the early onset of obesity and MS in children.

Methods: A systematic review for original articles between 1965 and June 2010 that focused on genes associated with obesity or MS in children was performed in PubMed. The search terms used were (((“Obesity”[Mesh]) OR “Metabolic Syndrome X/genetics”[Mesh]) OR “Insulin Resistance"[Mesh]) AND “Genes"[Mesh] AND ("humans"[MeSH Terms] AND ("infant" [MeSH Terms] OR "child"[MeSH Terms] OR "adolescent"[MeSH Terms])). A total of 346 articles of which 12 are revisions were considered.

Results: Regardless of 17 well-known genes involved in monogenic obesity, common variants near the melanocortin 4 receptor (MC4R) gene and a set of SNPs in the first intron of the FTO (fat mass and obesity associated) gene on chromosome 16q12.2 were consistently strongly associated with early-onset and severe obesity in children of European ancestry. PPARG, ADRB2, ADIPOQ and $F A A H$ gene variants were also associated with some obesity traits in children. Variants for the genes $A D R B 3, A P O B \mathrm{XbaI}$ and EcoRI restriction sites, APOE, IGFBP-1, INS VNTR, IRS-1, IRS-2, Kir6.2, LRP5, PAI-1, PIK3, PLIN4, PPARG, RAGE, TCF7L2, TFAP2B, and TNFalpha have been associated with features of MS and IRS. The UGT1A1 genotype is also a new risk factor for paediatric non alcoholic fatty liver disease (NAFLD). Recent data of our group also show that variants of MAP3K4, NTRK2, LEPR, PLIN and ZA20D2 genes are related to the early onset of MS in children.

Conclusions: Many gene variants are associated with multifactorial obesity and particularly some of them related to lipid metabolism and cell signaling are involved in the early presence of MS and IRS in children.

\section{4 \\ Effect of Tissue n-6/n-3 Fatty Acid Ratio on the Expression of Inflammatory Factors and Atherosclerotic Plaque Formation}

\author{
Jian-Bo Wan, Jing X. Kang* \\ Laboratory for Lipid Medicine and Technology, \\ Department of Medicine, Massachusetts General Hospital \\ and Harvard Medical School, Boston, Mass., USA
}

Background and Objectives: Although it has been suggested that a low ratio of $n-6 / n-3$ polyunsaturated fatty acids (PUFA) is more desirable in reducing the risk of atherosclerotic cardiovascular disease, the role of $n-6 / n-3$ fatty acid ratio in atherosclerotic lesion formation has not been well addressed in well-controlled experimental system. The fat- 1 transgenic mice, expressing an n-3 fatty acid desaturase, are capable of producing n-3 PUFA from n-6 PUFA and thereby have a low or balanced ratio of n-6/n-3 fatty acids in their tissues and organs. The aim of this study was to use the fat- 1 transgenic mouse model to determine the role of tissue $n-6 / n-3$ fatty acid ratio in atherosclerotic plaque formation.

Methods and Results: To generate apolipoprotein E-deficient plus fat-1 transgenic mice (apo $E^{-l-} \mid$ fat -1$)$, we crossed heterozygous fat-1 mice with $a p o E^{-1-}$ mice. After 14 weeks of feeding with an Western-type diet rich in n-6 PUFA, the apoE $E^{-1} /$ fat- 1 mice showed a lower or balanced n-6/n-3 fatty acid ratio in all organs and tissues tested, compared with $a p o E^{-/-}$mice. The atherosclerotic plaque in the entire aorta of apoE $E^{-1-} /$ fat-1 mice was remarkably smaller than that of apo $E^{-1-}$ littermate $(7.14 \pm 0.54 \%$ and $13.49 \pm 1.61 \%$, respectively). A significant reduction of interleukin-6 (IL-6) and prostaglandin E2 (PGE2) in both plasma and aorta culture medium were observed in apoE $E^{-/} /$fat -1 mice. Real time RT-PCR analysis indicated that the expression of intercellular adhesion molecule-1 (ICAM-1), monocyte chemoattractant protein-1 (MCP-1), IL-6 and cyclooxygenase-2 (COX-2) were all decreased in both aortas and circulating monocytes from apo $E^{-1-} /$ fat -1 mice, compared with $a p o E^{-/}$mice. In addition, the expression of nuclear factor- $\kappa \mathrm{B}(\mathrm{NF}-\kappa \mathrm{B}) / \mathrm{p} 65$ in the aorta and the recruitment of monocytes into atherosclerotic plaques were apparently reduced in apo $E^{-/-} /$fat-1 mice, compared with apo $E^{-/-}$littermates. There were no differences in plasma cholesterol, HDL and LDL levels between the two groups, except higher TG in apoE $E^{-1-}$ fat-1 mice.

Conclusion: These findings demonstrate that decreased tissue $\mathrm{n}-6 / \mathrm{n}-3$ fatty acid ratio reduced atherosclerotic plaque formation in $a p o E^{-/}$mice, associated with its anti-inflammatory effects rather than the lipid-lowering effect. 


\section{Epigenetics}

015

\section{Epigenomics: Understanding How Nutrition Modulates the Ageing Process}

\author{
Sofia Lisanti, Thomas von Zglinicki, John C. Mathers \\ Human Nutrition Research Centre, Centre for Integrated \\ Systems Biology of Ageing and Nutrition, Institute for \\ Ageing and Health, Newcastle University, Newcastle on \\ Tyne, UK
}

The worldwide increase in lifespan is evidence of considerable malleability in the ageing process. The challenge is to understand the factors influencing ageing so that strategies which facilitate healthy ageing, i.e. maintenance of the healthy ageing phenotype, can be maximised. There is growing evidence that nutrition and lifestyle may be key environmental determinants of ageing because they have profound effects on the genomic and cellular damage which appears to be a fundamental cause of the reduced function and increased risk of frailty which characterise physiological ageing.

During ageing, epigenetic marks including patterns of DNA methylation are altered and this may contribute to both altered gene expression and genomic instability. In addition, nutritional interventions affect epigenetic marks and such epigenomic mechanisms may mediate the influence of diet on the ageing process. Research priorities include i) characterisation key epigenetic changes which are causal for ageing and ii) identification of age-related genomic loci which respond to nutritional intervention.

Objectives: To assess the impact of ageing on genome-wide changes in DNA methylation and to identify genomic loci which are epigenetically labile in response to dietary restriction.

Methods: Genomic DNA was extracted from liver from young (3 months) and old (32 months) C57B16 mice and from mice aged 17 months which had been subjected to 3 months dietary restriction or fed normally. In preparation for whole genome methylation analysis, Methylated DNA ImmunoPrecipitation (MeDIP) was applied to genomic DNA fragments which were immunoprecipitated with a 5'-MethylCytidine antibody. The MeDIP/Input enrichment was estimated by qPCR of methylated regions/unmethylated regions.

Results: MeDIP proved very successful in preparing DNA for methylation array analysis. We observed that the enrichment differed when the ratio was calculated for the same methylated region over different unmethylated regions of housekeeping genes. This suggests that $\mathrm{CpG}$ islands in the promoters of one or more of these genes may become methylated during ageing. NimbleGen $\mathrm{CpG}$ methylation promoter arrays were used to screen the whole genome for loci whose methylation status was altered during ageing and we confirmed changes by Pyrosequencing.

Conclusions: MeDIP followed by genome-wide analysis by microarray proved highly satisfactory as an approach for identifying genetic loci which respond to both ageing and dietary intervention. We plan to integrate these genome-wide methylation data with genome-wide expression data to identify genes and pathways which are likely to be regulated epigenetically during ageing and where effects of ageing may be modulated by nutrition.
Funded by BBSRC and EPSRC via the Centre for Integrated Systems Biology of Ageing and Nutrition (CISBAN) (BB/ C008200/1).

\section{6 \\ Epigenetic Mechanisms in Obesity}

Robert A. Waterland

Departments of Pediatrics and Molecular \& Human

Genetics, Baylor College of Medicine, USDA/ARS

Children's Nutrition Research Center, Houston, Tex., USA

Transgenerational studies in agouti viable yellow mice (1) suggest that maternal obesity may interact with the developmental establishment of epigenetic gene regulatory mechanisms that affect body weight regulation. Epigenetics is the study of heritable changes in gene expression potential that are not mediated by DNA sequence alterations (2). Epigenetic mechanisms are susceptible to environmental influences during development (3); nutritional perturbation of epigenetic gene regulation therefore provides a mechanism by which maternal obesity could affect obesity susceptibility in her offrspring.

Epigenetic information is conveyed in mammals via synergistic interactions among mitotically-heritable patterns of DNA methylation, histone modifications, and various DNA-binding proteins. Because it often correlates with other regional epigenetic mechanisms like histone acetylation and methylation (4), we are focusing on DNA methylation as an overall indicator of locus-specific epigenetic alterations that regulate gene expression.

Epigenetic dysregulation can affect body weight regulation. It remains unclear, however, whether epigenetic mechanisms play an important role in the current obesity epidemic. As the primary site integrating the regulation of energy balance, the hypothalamus is an excellent candidate tissue in which to focus initial studies. The suckling period in the mouse is a critical period for morphological and functional development of the hypothalamus (5). To test the hypothesis that the hypothalamus is undergoing epigenetic maturation in the early postnatal period, we have compared P21 vs. P0 mouse hypothalamus by methylation-specific amplification and microarray hybridization (6). I will present data from these ongoing experiments, and discuss current research priorities.

\section{References}

1. Waterland RA, Travisano M, Tahiliani KG, Rached MT, Mirza S. Methyl donor supplementation prevents transgenerational amplification of obesity. Int J Obes (Lond) 2008;32:1373-9.

2. Bird A. DNA methylation patterns and epigenetic memory. Genes Dev 2002;16:6-21.

3. Waterland RA, Jirtle RL. Early nutrition, epigenetic changes at transposons and imprinted genes, and enhanced susceptibility to adult chronic diseases. Nutrition 2004;20:63-68.

4. Jaenisch R, Bird A. Epigenetic regulation of gene expression: how the genome integrates intrinsic and environmental signals. Nat Genet 2003;33 Suppl:245-54.

5. Bouret SG, Draper SJ, Simerly RB. Trophic action of leptin on hypothalamic neurons that regulate feeding. Science 2004;304:108-10.

6. Shen L, Kondo Y, Guo Y, et al. Genome-wide profiling of DNA methylation reveals a class of normally methylated $\mathrm{CpG}$ island promoters. PLoS Genet 2007;3:2023-36. 


\section{7 \\ Interactions Between Diet and Epigenetics: Implications for Cancer Prevention}

\section{Sharon A. Ross}

Nutritional Science Research Group, Division of Cancer

Prevention, $\mathrm{NCl}, \mathrm{NIH}$, Bethesda, Md., USA

Evidence indicates that epigenetic mechanisms influence gene expression and chromatin stability to impact the regulation of cellular pathways and ultimately phenotype of the organism. Epigenetic mechanisms include DNA methylation, histone posttranslational modifications, non-coding RNAs, and other factors/proteins (i.e., polycomb repressive complexes) that regulate chromatin structure and dynamics. The role of aberrant epigenetic regulation in the etiology of disease, including cancer development and progression, has been increasingly recognized in recent years. In cancer cells, the transcriptional silencing of tumor-suppressor genes by CpG-islandpromoter hypermethylation is thought to be an early response in the tumorigenic process. Such site specific DNA hypermethylation along with widespread global DNA hypomethylation, which is associated with chromatin instability, are common characteristics of tumor cells. Epigenetic regulatory processes- because they appear to be susceptible to changes by environmental factors- offer potential mechanistic explanations for how diet may modify cancer risk and tumor behavior. In fact, many studies provide evidence that part of the cancer inhibiting properties associated with several dietary components may relate to changes in epigenetic processes. For example, sulforaphane (SFN) an isothiocyanate found in cruciferous vegetables, such as broccoli and broccoli sprouts, has displayed anticancer properties through epigenetic mechanisms. Specifically, SFN has been shown to inhibit histone deacetylase activity in human colon and prostate cancer cells, which increased global histone acetylation and local histone acetylation status on the promoter region of the $\mathrm{p} 21$ gene and enhanced gene expression of this tumor suppressor gene. Because studies have shown that dietary fruits and vegetables and the dietary constituents they contain may reduce risk of lung cancer, researchers recently evaluated whether diet and multivitamin use influenced the prevalence of gene promoter methylation in cells exfoliated from the aerodigestive tract of current and former smokers. Consumption of leafy green vegetables and folate as well as current use of multivitamins was found to be significantly inversely associated with promoter methylation of a panel of genes. This study supports the concept that dietary interventions to prevent lung cancer could be explored based on their ability to affect reprogramming of the epigenome. Understanding how diet and nutrition participate in modifying gene expression is complex given the range of food choices, the diversity of nutrient intakes, the individual differences in genetic backgrounds and intestinal physiological environments where food is metabolized, but should provide worthwhile insights on strategies to prevent cancer.

\section{8}

\section{The Effect of Nutrition on the Epigenome and the Implications for Human Disease}

\author{
Karen Lillycrop, Graham Burdge \\ Institute of Developmental Sciences, University of \\ Southampton, UK
}

Epidemiological studies have shown that a poor intra-uterine environment induced by unbalanced maternal diet, body composition, placental insufficiency or endocrine factors induces a phenotype in the offspring that is characterized by an increased risk of developing chronic non-communicable diseases in later life. These include obesity, type II diabetes, cardiovascular disease and some forms of cancer. This has been replicated in a range of animal models. The association between the quality of the prenatal environment and disease risk may reflect mismatch between the future environment 'predicted' by the embryo/ fetus, based on signals from the mother during gestation, and the actual environment experienced in later life. The mechanism by which cues about nutrient availability in the postnatal environment lead to induction of stable phenotypes is beginning to be understood and involves the epigenetic regulation of specific genes.

We have shown that modest changes to the maternal intake of a macronutrients during pregnancy induces stable changes to the epigenetic regulation of specific genes, leading to long term alterations in the metabolism and physiology of the offspring. Thus these findings support the concept that epigenetic processes allow the fetus to adapt to nutritional cues from the mother, while inappropriate adaptations may predispose an individual to increased risk of a range of non-communicable diseases. Such epigenetic changes can be modified by supplementation with folic acid or methyl donors during pregnancy, or folic acid after weaning. The ability to prevent or reverse induced epigenetic marks opens the possibility of reducing the prevalence of chronic diseases in later life.

\section{Obesity}

\section{9 \\ An Adipocyte-Driven Mechanism Underlying Weight Regain (Yo-Yo Effect) in Human Overweight Subjects}

\section{Edwin C.M. Mariman}

Department of Human Biology, Maastricht University, Maastricht, The Netherlands.

Objectives: Obesity is a risk factor for diseases such as type II diabetes, cardiovascular disorders, reduced fertility and certain types of cancer. In essence, the treatment of obesity is simple: a weight loss of $5-10 \%$ already reduces the obesity-related health risks considerably. In real terms, however, obesity treatment is far from simple. One year after weight loss between $35 \%$ and $80 \%$ of subjects has returned 
to the original weight or even beyond. This so-called 'yo-yo effect' is the true problem of obesity. The high risk for weight regain is traditionally ascribed to psychosocial factors. In addition, a drop in leptin level may increase the risk for weight regain. Recently we have obtained new insight from data indicating that also a cellular mechanism accounts for the poor success of weight maintenance after weight loss.

Methods: Eight overweight/obese persons lost $\sim 10 \mathrm{~kg}$ by means of 5 weeks VLCD followed by a weight maintenance period of 3 weeks. Before and after the intervention subcutaneous adipose tissue was collected. Using a proteome subtraction method we analysed specifically the proteins of adipocytes in vivo. In addition, targeted analysis was done with Western blotting.

Findings: After weight loss and return to a state of energy balance, adipocytes prepare for re-storing fat. At the same time, differential expression was observed of proteins belonging to the so-called stress proteome suggesting induction of cellular stress by mechanical forces.

Interpretation: During weight loss, cellular stress develops in adipocytes possibly due to the traction forces between the membrane of the shrinking cell and the more rigid ECM. This promotes upregulation of the glucose uptake machinery and lipid handling proteins preparing the adipocytes for re-storage of fat, which would release the adipocytes from stress. However, for the carrier of the adipocytes, this means weight regain.

\section{0 \\ Nutrigenomics for Obesity Prevention and Treatment}

\section{David M. Mutch \\ Department of Human Health \& Nutritional Sciences, University of Guelph, Guelph, Ontario, Canada}

Over the past thirty years the prevalence of both adult and childhood obesity has steadily increased to epidemic proportions. Lifestyle interventions (i.e. caloric restriction and increased physical activity) remain the most common recommendations for controlling human obesity; however, considerable inter-individual variability exists regarding successful weight management. Therefore alternate approaches must be considered and tested if we are to turn the tide in our fight against obesity. Nutrigenomics is poised to have a significant impact on the prevention and treatment of obesity by recommending lifestyle modifications based on an individual's genetic information. Much of our recent holistic understanding regarding the role of adipose tissue in obesity stems from the use of using modern molecular biology tools, such as the microarray. This presentation will highlight emerging research in which gene expression profiling has been used to improve our understanding of the variability in weight loss and weight maintenance observed between individuals during a diet intervention protocol. The ability to identify obese individuals who will successfully respond to dietary interventions will revolutionize disease management. The first studies discussed will demonstrate how adipose gene expression profiles can be used to predict weight loss. These works demonstrate that gene expression profiling prior to caloric restriction can differentiate responders from non-responders, as well as serve as a weak predictor of subjects des- tined to lose weight. A more recent study further demonstrates that individuals who can successfully maintain their weight have a distinct molecular response to a low-calorie diet in comparison to individuals who regain weight. While interpreting the vast quantity of data generated by microarray experiments continues to challenge and the clinical utility of these findings remains in their infancy, the knowledge already generated by these studies demonstrates that nutrigenomics has taken important steps towards realising its promise of personalized nutrition.

021

\section{Eating Behaviour Questionnaires in Families: Application in Genetic Epidemiology Studies of Obesity}

\section{J.L. Santos}

Department of Nutrition, Diabetes and Metabolism. School of Medicine. Pontificia Universidad Católica de Chile, Santiago, Chile

Childhood eating behaviour questionnaires are increasingly being used as psychometric tools in epidemiologic studies of human obesity. Subscales of the Child Eating Behaviour Questionnaire (CEBQ) indicating positive inclinations to higher intake (for example, enjoyment of food or food responsiveness) are strongly associated with increased weight in children. On the contrary, food-avoidant subscales (such as satiety responsiveness or slowness in eating) are inversely associated with obesity. Other questionnaires based of the concept of "eating in the absence of hunger" (EAH) have been developed, as well as direct measurements of eating after the consumption of usual lunch. In adults, the Three Factor Eating Questionnaire (TFEQ) has also been used to assess eating dimensions of uncontrolled eating, emotional eating and cognitive restriction. In this research work, I show results from epidemiologic studies integrating eating behaviour patterns (CEBQ, EAH and TFEQ-R18), anthropometric measurements and genetic data in Chilean children and their parents. Two different types of genetic markers were evaluated: 1) single nucleotide polymorphisms of the melanocortin 3 and 4 receptor genes (MC3R and MC4R) and 2) copy-number variation (CNV) of the salivary amylase gene (AMY1). Mutations in MC4R are the main cause of rare forms of monogenic obesity. Additionally, genomewide association studies showed that a common polymorphism located $188 \mathrm{~kb}$ downstream of MC4R (rs17782313) has been consistently associated with body mass index in large epidemiological studies. It has been also suggested that the C-allele of rs17782313 is associated with specific eating behavior patterns. Common functional missense variations in the MC3R gene (Thr6Lys and Val81Ile) have been previously associated with childhood obesity and have a potential role in energy intake. On the other hand, the human salivary amylase gene AMY1 shows extensive CNV which is directly proportional to its protein expression in saliva. Variation in this gene has been related to psychosocial stress and increased satiety. It is concluded that genetic variation in MC3R, MC4R or AMY1 genes may have different effects on eating behaviour scores acting at different levels.

J Nutrigenet Nutrigenomics 2010;3:49-124 


\section{2}

\section{Genetics of Common of Obesity - Recent Progress Through Genome-Wide Association Studies and Future Challenges}

Ruth J.F. Loos

MRC Epidemiology Unit, Institute of Metabolic Science, Cambridge, UK

Obesity is a major risk factor for many common diseases. While lifestyle changes have driven its prevalence to epidemic proportions, heritability studies suggest that genetic factors explain $40 \%$ to $70 \%$ of the variation in BMI and in risk of obesity. Yet, despite a relatively high heritability, the search for obesity susceptibility genes has been an arduous task with limited success in the past 15 years.

Since 2005, however, the pace of gene discovery has dramatically increased through genome-wide association studies (GWAs), which have proven to be successful for various common diseases and traits, including obesity-related traits. This presentation reviews the latest progress in the obesity genetics field and highlights the promise and the future challenges of the genome-wide association approach.

So far, four waves of large-scale high-density genome-wide association studies have discovered $\sim 40$ obesity susceptibility, most of which were previously unanticipated. Firstly, in 2007, two GWAs led to the discovery of FTO as the first major obesity-susceptibility gene. Next, in 2008, the second obesity-susceptibility locus mapped near $M C 4 R$ and was identified through a large-scale GWA meta-analysis including $>16,000$ individuals as part of the GIANT (Genetic Investigation of ANtropometric Traits) consortium. In 2009, the GIANT consortium extended its GWA meta-analysis to $>32,000$ individuals which led to the identification of six new loci robustly associated with BMI. At the same time, the deCODE group identified with a similar sized GWA meta-analyses, eight new loci of which four loci had also been identified by the GIANT consortium, whereas four loci were new. Two GWAs for waist circumference and one GWA for early-onset obesity identified an additional seven loci consistently associated with obesity-related traits. Currently, a fourth wave of gene-discovery in ongoing as part of a further extension of the GIANT consortium, including $>123,000$ individuals. The meta-analysis is set to identify $\sim 20$ new loci robustly associated with BMI. Taken together, four waves of GWAs have identified at least 35 obesity susceptibility loci in less then four years time.

Each of these obesity-susceptibility loci has a modest effect and when considered combined they explain $<5 \%$ of the variation in BMI and their predictive value of obesity is limited. Yet, the loci combined allow identifying individuals who are susceptible to (who carry many risk loci) and those who seem protected (who carry none or few risk loci) from obesity and the difference in weight between these two groups of individuals can amount to $>4 \mathrm{~kg}$ solely due to a difference in genetic background. Studies have shown that this genetic susceptibility can be attenuated by living a physically active lifestyle. While GWAs have typically been performed in adults, most of the new obesity susceptibility loci have already an effect on childhood BMI, but not on birth weight.

Currently, the two major challenges are to [1] find more obesitysusceptibility variants, which will require further large-scale data integration and more refined analyses, and to [2] examine the established loci in detail to identify the causal variants and genes, which will allow investigating the physiology that underlies common obesity.
Preliminary information about the underlying biology of these new loci provides support for a neuronal basis to obesity-susceptibility, and also suggests a role for apoptosis and retinoic acid biology. While so far little experimental data is available, these new loci promise to enhance our knowledge of underlying biology, open new avenues for functional research, and may provide guidance in developing or improving interventions for obesity.

\section{Diabetes}

\section{3 \\ Biogenic Amines Substrates of SSAO Influencing Adipose Tissue Gene Expression and Glycaemic Control in Obese and Diabetic Mice}

\author{
Zsuzsa Iffiú-Soltész, Estelle Wanecq, Josep Mercader, \\ Christian Carpéné \\ INSERM, U858, I2MR, IFR 150, University Toulouse III, \\ Toulouse, France
}

Introduction: benzylamine (Bza) is one of the dietary amines found in food and beverages (such as tyramine, polyamines...), and it has been reported to be present in fruits and vegetables $(<10 \mathrm{ppm})$, while trimethylbenzylamine is a flavoring agent (added at $\leq 10 \mathrm{ppm}$ in baked goods). Bza is more known as an exogenous substrate of primary amine oxidases, a family of copper-containing enzymes, including semicarbazide-sentive amine oxidase (SSAO). In vitro studies have shown that, when combined with vanadium, Bza mimics several insulin actions glucose in rat adipocytes: glucose uptake and lipogenesis stimulation, lipolysis inhibition. Bza improves glucose tolerance when injected in mice at $7 \mu \mathrm{mol} / \mathrm{kg}$. Our objective was to study whether the chronic administration of Bza was able to improve glucose utilization in diabetic mice.

Methods and Materials: just after weaning, homozygous db-/mice and their $\mathrm{db}+/$ ? lean littermates received benzylamine at $0.5 \%$ in the drinking water, while controls had ad lib. acces to water and chow during the same period, from 4 to 11 weeks of age.

Results and Conclusions: $\mathrm{db}+/$ ? were normoglycemic throughout the study, and did not exhibit noticeable change between untreated and Bza-drinking groups. In untreated $\mathrm{db}-/-$ mice, the non-fasting blood glucose increased continuously with body mass and reached $5.5 \mathrm{~g} / \mathrm{l}$ at 11 weeks of age. In Bza-drinking mice, this increase was delayed and limited to $4.0 \mathrm{~g} / \mathrm{l}$, while food consumption was reduced by $15 \%$. Water consumption and urine emission were dramatically reduced, leading to an almost abolished urinary glucose production. The fasting plasma levels of glucose and triglycerides were reduced in Bza-drinking db-/- mice. Accordingly, oxidative stress circulating markers and expression of diabetes-related genes in adipose tissue (e.g. F4/80, apelin) were almost normalized in Bza-drinking db-/mice. Thus, Bza supplementation has beneficial action on glucose handling in the $\mathrm{db}-/-$ mouse, and can be considered as a potential antidiabetic approach, which involves SSAO activation in adipocytes. 


\section{4 \\ Sex and Diet Dictate the Metabolic Syndrome Epigenetic Programming in the Placenta}

\author{
Claudine Junien \\ INRA, UMR1198, UMR INRA / ENV Maisons-Alfort/CNRS: \\ Biologie du Développement et Reproduction, (ENV \\ Maisons-Alfort; CNRS), Centre de recherche de Jouy-en- \\ Josas, Jouy-en-Josas, France
}

The ways in which epigenetic modifications fix the effects of early environmental events, ensuring sustained responses to transient stimuli, which result into modified gene expression patterns and phenotypes later in life, is a topic of considerable interest. A number of studies focusing on the developmental origin of health and disease $(\mathrm{DOHaD})$ and metabolic programming have identified links between early nutrition, epigenetic processes and long-term illness. Recent studies demonstrate an obvious sexual dimorphism both for programming trajectories and in response to the same environmental insult. The placenta plays a central role in the development of the fetus by modulating the supply of nutrients and oxygen throughout pregnancy. The placenta adapts to the presence of a maternal pathophysiology in a sexually dimorphic manner which results in differences in fetal growth between males and females. In order to better characterize the placenta as a programming agent for obesity, type 2 diabetes and cardiovascular disease we used microarrays, RT-qPCR analysis, epigenetic techniques, immunohistochemistry, and in situ hybridization to investigate whether a high-fat diet (HFD) during pregnancy modified the global expression of murine genes, imprinted genes and local and global DNA methylation and histone modifications patterns in the mouse placenta. A HFD during gestation triggers sex-specific epigenetic alterations within $\mathrm{CpG}$ and throughout the genome, together with the deregulation of clusters of imprinted genes important in the control of many cellular, metabolic and physiological functions potentially involved in adaptation/evolution. Microarray analysis showed that sexual dimorphism exists regardless of diet. Males and females placentae use different pathways to cope with the HFD. These findings highlight the importance of studying both sexes in epidemiological protocols and dietary interventions. Taking into account sex-specific effects should allow us to identify a significant proportion of the genes that contribute to risk for complex diseases such as obesity, type 2 diabetes and cardiovascular disease.

\section{5 \\ Diet, Gene Polymorphisms and The Metabolic Syndrome}

\section{Denis Lairon \\ Lipid nutrients and prevention of metabolic diseases, Faculty of medicine, Marseille, France}

The metabolic syndrome (MetS) is a common, multi-component condition characterized by abdominal obesity, insulin resistance, dyslipidaemia, and hypertension. Patients with MetS present an increased risk of type 2 diabetes mellitus, cardiovascular disease and athero- sclerosis. The prevalence of the MetS is increasing dramatically worldwide, both in developped and developing countries, following the increasing prevalence of obesity.

MetS probably results from the interaction of a predisposing genetic background with several environmental factors. In particular, dietary habits have been favorably associated with some of these factors included in the definition of metabolic syndrome and along with other nutrients, the quantity and quality of lipid intake has been involved.

We aimed to study the possible interaction between dietary fat and genetic variants. The initial attempt was through the follow-up of the 3 -month dietary intervention in the MEDI-Rivage cohort (169 subjects with traits of the MetS; 22 SNPs). In the framework of the EU-funded LIPGENE integrated project (2004-09), this was then performed through two complementary approaches : first, a nested prospective matched MetS case-control study (877 pairs; 806 SNPs) with 7.5 years follow-up and secondly, a multicentric 3-month intervention study in 337 subjects with MetS which were randomly assigned to one of four iso-energetic diets (rich in SFA, MUFA, low-fat, lowfat + EPA/DHA).

Some results obtained during these studies are presented. Interactions between diets (especially fatty acids) and some gene variants (SNPs in ApoB, MTP, FABP2, ApoE, C3, STAT3, TCF7L2, ACSL1, ACCB, LEPR, ADIPOQ, IL-6, LTA, TNF $\alpha$ ) have been found. Overall, the relative importance of fatty acids and gene variation in MetS development was evaluated using the Random forest approach.

Taken together, these new data along with other ones allow toconclude that both dietary fatty acid intakes, endogenous fatty acid metabolism and gene variants play a role in the MetS and its phenotypic components.

\section{6 \\ Gene-Environment Interactions in the Development of Obesity and Type 2 Diabetes}

Frank B. Hu

Harvard School of Public Health, Harvard Medical School, USA

Obesity and type 2 diabetes have become global epidemics. Both environmental (e.g. physical activity and diet) and genetic factors are involved in the development of these conditions. Rapid changes in diet and lifestyle are believed to be the driving forces behind these epidemics. In developing countries, concurrent with the rapid economic development, people are eating a more Westernized diet of highly processed foods and moving away from their traditional active lifestyle. In the past decade, studies using traditional linkage analysis and candidate-gene association approach have found dozens of genes harboring common variants that were related to the common-form type 2 diabetes. However, most reported associations were not replicated except for TCF7L2, PPARG, and KCNJ11. Since 2007, several genome-wide association (GWA) studies have identified more than 30 new genetic variants that predict type 2 diabetes. Recent data from observational studies and randomized clinical trials suggest interactions between dietary and lifestyle factors in relation to obesity and diabetes. In particular, several studies found a significant interaction 
between FTO variants and physical activity on obesity - higher physical activity attenuates the effects of FTO variants. Lifestyle intervention including diet and exercise has been shown to reduce the effects of TCF7L2 variants. In addition, subjects with greater genetic predisposition (reflected by a higher genetic risk factor) are more susceptible to deleterious effects of a Western dietary pattern on type 2 diabetes. These results, albeit preliminary, suggest an interplay between environmental and genetic risk factors in the development of obesity and type 2 diabetes.

\section{Inflammation}

\section{7 \\ Hypoxia and the Inflammatory Response in Adipose Tissue in Obesity}

Paul Trayhurn

Obesity Biology Research Unit, University of Liverpool, Liverpool, UK and Clore Laboratory, University of

Buckingham, Buckingham, UK

White adipocytes are major endocrine and secretory cells, releasing a range of protein hormones and factors - the adipokines. Many adipokines are linked to the inflammatory response, and inflammation within the enlarged adipose mass underlies the development of the disorders linked to obesity. We have proposed that local hypoxia develops in white fat as the tissue expands, large adipocytes becoming $\mathrm{O}_{2}$-deprived as their distance from the vasculature increases, this underlying the inflammatory response. Direct evidence for hypoxia in adipose tissue in obesity has recently been obtained in mice and humans. Candidate gene studies with adipocytes in culture have shown that the expression and secretion of several key inflammationrelated adipokines, including IL-6, leptin, Angplt4 and VEGF are stimulated by low $\mathrm{pO}_{2}$; the production of adiponectin with its antiinflammatory action is, however, inhibited. PCR arrays have identified a member of the metallothionein family of metal binding proteins, MT-3, as being highly hypoxia-inducible in human adipocytes, while microarray studies have demonstrated that in response to low $\mathrm{O}_{2}$ the expression of $>650$ genes is upregulated and $>600$ downregulated. These include the facilitative glucose transporter GLUT1, expression of which increases, and there is a substantial rise in GLUT1 protein. This is linked to a hypoxia-stimulated increase in glucose uptake, and lactate release is also increased indicative of a switch to glycolytic metabolism. Synthesis of the monocarboxylate transporter, MCT1, is stimulated in adipocytes by hypoxia, enabling increased lactate removal from hypoxic fat cells. Importantly, hypoxia induces insulin resistance in adipocytes, this involving the loss of the insulin-sensitive glucose transporter GLUT4. Other cells within adipose tissue also respond to hypoxia; for example, in preadipocytes, the synthesis of leptin is induced. It is proposed that hypoxia has a pervasive effect on adipocyte function and is central to the dysregulation of adipose tissue in obesity that underlies insulin resistance and the metabolic syndrome.

\section{8 \\ The Role of Inflammation in the Regulation of the Mass and Cellularity of Human Adipose Tissue}

Peter Arner

Karolinska Institutet at the Department of Medicine, Karolinska University Hospital, Stockholm, Sweden

Human adipose tissue is in a dynamic state with a marked turnover of fat cells. About $10 \%$ of the total fat cell pool is renewed due to adipogenesis and cell death throughout the adult human life (1). The mechanisms responsible for the regulation for this turnover are not clear.

An important feature of the expanding adipose tissue mass in obesity is local low grade inflammation. This inflammation has until recently been considered as pernicious and at last in part responsible for adipose dysfunction linking this tissue to insulin resistance, hypertension, dyslipidemia and other metabolic abnormalities among the obese. However, recent data suggest that adipose inflammation also may play a physiological role (2). In adipose tissue of lean healthy subjects there is a strong correlation between the secretion of tumour necrosis factor alpha and the cellularity (hyperplasia/hypertrophy) or total amount of adipose tissue. Such a relationship is not found for other inflammatory proteins or adipokines such as leptin or adiponectin. Thus, local inflammation may regulate the normal mass and cellularity of adipose tissue in addition to its pathophysiological role occurring when excess fat mass is present.

We have started a system biology study to elucidate the mechanisms regulating the production and secretion of inflammatory proteins in human adipose tissue in the non-obese and obese states. Preliminary results from these investigations will be presented.

1. Spalding KL, Arner E, Westermark PO, Bernard S, Buchholz BA, German OI, Blomqvist L, Hoffstedt J, Näslund E, Britton T, Concha H, Hassan M, Rydén M, Frisén J, Arner P. Dynamics of fat cell turnover in humans. Nature 453:783, 2008

2. Arner E, Rydén M, Arner P. Tumor necrosis factor alpha and regulation of adipose tissue. Letter N Engl J Med, 362(12):1151-3, 2010.

\section{9 \\ Nutritional Genomics of Adipose Tissue in Obesity and Insulin Resistance: Role of $\mathbf{n - 3}$ PUFA}

María Jesús Moreno-Aliaga

Department of Nutrition, Food Sciences, Physiology and Toxicology, University of Navarra, Pamplona, Spain

Background and Aims: Low-grade inflammation is a key factor in the development of metabolic syndrome in obese subjects. The expanding adipose tissue makes a substantial contribution to the development of obesity-linked inflammation via dysregulated secretion of pro-inflammatory cytokines, chemokines and adipokines and the reduction of anti-inflammatory adipokines such as adiponectin. n-3 PUFA have been shown to prevent and/or ameliorate inflammation in key metabolic organs including adipose tissue, having protective effects in a range of chronic inflammatory conditions, including 
obesity and insulin resistance. The aim of this contribution is to review the current knowledge on the genetic and molecular mechanisms by which n-3 PUFAs are able to unlink obesity-induced inflammation and insulin resistance focusing mainly in white adipose tissue.

Methods: In the last years the application of nutrigenomics techniques, including large-scale profiling of genes, proteins, and metabolites are strongly facilitating a detailed description of the molecular processes involved in n-3 PUFA actions in obesity and insulin resistance.

Results: n-3 PUFA anti-inflammatory effects are mediated by a decrease in inflammatory mediators such as arachidonic acid-derived eicosanoids and inflammatory cytokines. Moreover, n-3 PUFA serve as substrates for the conversion to a novel series of lipid mediators designated resolvins and protectins, with potent anti-inflammatory and pro-resolving properties. n-3 PUFA may also blunt adipose tissue inflammation by acting as endogenous ligands for the peroxisome proliferator-activator receptors (PPARs) in adipocytes and macrophages. Furthermore, n-3 PUFA are able to modulate the secretion of bioactive adipokines (including leptin, adiponectin, visfatin and apelin) related with inflammation and insulin sensitivity. In addition, the n-3 EPA prevents TNF- $\alpha$-induced activation of ERK $1 / 2$ and NF- $\kappa B$ in adipocytes. $n-3$ PUFA have been also shown to upregulate mitochondrial biogenesis and induce beta-oxidation in WAT probably by stimulating AMPK.

Conclusion: Although clearly at the beginning, nutrigenomics will allow to decipher the molecular networks regulated by n-3 PUFA, and other bioactive food compounds and their potential application for the treatment of obesity and its related disorders.

\section{Cancer}

\section{0 \\ Emerging Topics in Nutrigenomics and Cancer Prevention}

\author{
J.A. Milner \\ Nutritional Sciences Research Group, Division Cancer \\ Prevention, National Cancer Institute, National Institutes \\ of Health, Bethesda, Md., USA
}

All indicators point to the likelihood of global increases in cancer risk during the next 20 years unless effective preventative strategies are implemented. It is also increasingly apparent that risk may be decreased, unchanged or elevated following exposure to a similar amount of a food component and thus pointing to the need for personalized approaches to diet and cancer prevention. Understanding nutrigenomics is fundamental to characterizing the amount of a food or its component(s) that are needed to bring about a desired effect, while minimizing ill consequences. While there is evidence that genetics and epigenetics can influence the response to foods/components, specific changes in these have not been, in most cases, confirmed/validated, nor have they been correlated with a change in a specific human cancer related process. The effectiveness of a given exposure to a bioactive food component must depend on its binding affinity to its molecular target. While elastase may be a target for indole 3-carbinol, tubulin for isothiocyanates, and histone deacetylase for allyl sulfurs, few systemic studies have conducted in vivo. Fewer have correlated binding affinities with a change in a cancer related process and ultimately a phenotypic change. Far too little information exists about the speciation of a food component that accounts for a target change. Evidence will be presented that the gastrointestinal microflora can influence the types of compounds formed from foods such as pomegranate and the activity of an enzyme such as aromatase. Exaggerated intakes of both essential and non-essential nutrients can lead to unpredicted side effects. Personalized nutrigenomics strategies coupled with knowledge about appropriate nutrient exposures are critical for identifying vulnerable individuals. Undeniably, nutrigenomics will assist in unraveling the importance of diet in complex physiological conditions including cancer, but are also not without its own trials, tribulations and uncertainties. Continued research in nutrigenomics will lead to a personalized approach to optimizing food consumption patterns and health.

\section{1 \\ Mechanisms of Hepatocarcinogenesis Associated with the Impairment of Methionine Metabolism}

\author{
Enrique Santamaría, Virginia Sánchez-Quiles, \\ Joaquín Fernández-Irigoyen, Javier Muñoz, Jesús Prieto, \\ Fernando J. Corrales \\ Center for Applied Medical Research (CIMA) and \\ University of Navarra, Dept. Hepatology and Gene \\ Therapy, Proteomics Laboratory, Pamplona, Spain
}

Introduction: Methionine is an essential aminoacid that is metabolized mainly in the liver. In addition to protein synthesis, methionine is used as a precursor for S-adenosylmethionine (AdoMet) production, a reaction catalyzed by the enzyme methionine adenosyltransferase (MAT). AdoMet homeostasis is central to preserve the differentiated phenotype of hepatocytes. The aim of the present study is to elucidate mechanisms underlying the progression of liver cancer in association with deficient liver AdoMet metabolism, a common trait of liver diseases.

Results: Proteomic analyses allowed the identification of hundreds of differential proteins in the liver of MAT deficient mice, which showed impairment of different metabolic, cell signalling, proliferation and survival pathways. In particular, we focused our attention on prohibitin (PHB1), a protein involved in the preservation of mitochondrial function that also regulates gene expression in the nucleus through its interaction with Rb, E2F1 and others. Downregulation of Erlin 2 in deficient PHB1 cells suggest ER stress that, in turn, might participate in the apoptotic response of HCC cells to Phb1 silencing. In agreement to this hypothesis, we found a fall of IGFBP1 levels, increased CHOP levels, PARP cleavage and activation of caspase 12 and downstream caspase 7. ER stress parallels proteasome malfunction, as evidenced by the down-regulation of proteasome activator complex subunit 2 and stathmin, leading to the accumulation of missfolded and ubiquitinated proteins. 
Conclusions: A deficient AdoMet synthesis in the liver induced non-alcoholic steatohepatitis and HCC in mice through mechanisms involving the impairment of metabolic, survival and proliferative pathways. Deletion of PHB1 resulted in proteasome malfunction, ER stress-induced apoptosis, inhibition of cell proliferation through a mechanism dependent on NFKB and reduced the invasive potential of human HCC cells. Moreover, we provide a collection of potential biomarkers that may prove to be useful in the management of liver diseased patients.

\section{2 \\ Nuclear Receptors, Nutrients and Intestinal Tumorigenesis}

Antonio Moschetta

Laboratory of Lipid Metabolism and Cancer, Department of Translational Pharmacology, Consorzio Mario Negri Sud, Santa Maria Imbaro, Chieti and Clinica Medica "A. Murri", University of Bari, Bari, Italy

PGC1 $\alpha$ is a nuclear receptor coactivator able to induce mitochondrial biogenesis, oxidative phosphorylation (OXPHOS) and fatty acid $\beta$-oxidation with the final aim of providing a more efficient pathway for aerobic energy production ( Kelly, D.P. and Scarpulla, R.C., 2004, Genes Dev. 18, 357-368; Lehman, J.J. et al., 2000, J. Clin. Invest. $106,847-856$ ). In the continuously renewed intestinal epithelium, highly glycolytic proliferative cells in the crypts migrate to the villus axis and differentiate into mature enterocytes shifting their metabolism from glycolysis to OXPHOS and finally undergoing apoptosis (Clevers, H. 2006, Cell 127, 469-480; Clevers, H., 2004, Cancer Cell 5, 5-6; Pitari, G.M. et al., 2007, Clin. Pharmacol. Ther. 82, 441-447 ). Here we show that in the intestinal epithelial surface, PGC1 $\alpha$ drives mitochondrial biogenesis and respiratory capacity in presence of reduced antioxidant enzyme activities, thus determining the accumulation of reactive oxygen species (ROS) and fostering the fate of enterocytes toward apoptosis. We also show that PGC1 $\alpha$ progressively disappears in intestinal tumors. This observation strongly recalls the Warburg theory (Warburg, O., 1956, Science 123, 309314 ) according to which cancer cells even in the presence of oxygen prefer glycolysis in spite of OXPHOS. We then hypothesized that the transcriptional activation of PGC1 $\alpha$ in the intestine, with its doubled potential to drive metabolism toward OXPHOS and to determine oxidative stress, could revert the intestinal cancer cell phenotype. Indeed, PGC1 $\alpha$ over-expression in vivo in both Xenografted mice and intestinal specific transgenic PGC1 $\alpha$ overexpressing mice produces a significant block in tumor growth rate associated with a strong increase in apoptosis. Moreover, the complete absence of PGC1 $\alpha$ in PGC1 $1 \alpha \mathrm{KO}$ mice produces an intestinal tumor susceptible phenotype. Thus, combining gain and loss of function genetic approaches in human cells and mouse models of intestinal cancer, we present a novel intriguing scenario whereby $\mathrm{PGC} 1 \alpha$ protects against tumorigenesis by promoting mitochondrial-mediated apoptosis via ROS production.

\section{3}

\section{Dietary Mutagenesis, Genes and Cancer}

Lynnette R. Ferguson ${ }^{1,2}$

${ }^{1}$ Discipline of Nutrition, Faculty of Medical \& Health Sciences, The University of Auckland, Auckland, New Zealand, ${ }^{2}$ Nutrigenomics New Zealand, New Zealand

It has been estimated that approximately one third of cancers are linked to diet, and mutagenic effects of diet are a major cause of these cancers. A range of technologies, from in vitro mutagenicity tests to molecular epidemiology, have associated dietary cancers with mutagenic carcinogens, such as aflatoxin B1 and benzo[a]pyrene. Effects of diet in stimulating chronic inflammation may lead to reactive species and mutation as a secondary consequence, while dietary deficiencies and nutrient imbalances are also pro-mutagenic. Nutrients play a key role in cell signalling and may have profound influences on gene expression in the absence of permanent DNA changes. The role of variation in genes for DNA repair and xenobiotic metabolism has been extensively studied as a modulating factor in dietary carcinogenesis. However, genome-wide association studies have highlighted new pathways such as JAK/STAT signalling that strongly influence genomic instability, and also modulate cellular responses to dietary mutagens. Next-generation DNA sequencing and advanced technologies for studying epigenetic changes, will enable a more sophisticated understanding of the relative role of genes and diet in modulating mutagenesis and carcinogenesis in humans. 
Journal of

Nutrigenetics
Nutrigenomics
Addendum to:

J Nutrigenet Nutrigenomics 2010;3:49-124
Published online: November 17, 2010

\section{4 \\ Promoting Resolution of Inflammation with a Novel Genus of EFA-Derived Pro-Resolving Mediators}

\section{Charles N. Serhan}

Center for Experimental Therapeutics and Reperfusion Injury, Department of Anesthesia, Perioperative and Pain Medicine, Harvard Institutes of Medicine, BWH and Harvard Medical School, Boston, Mass., USA

Mechanisms in timely resolution of the acute response can give new insights in host defense. Using a systems approach with self-limited inflammatory exudates to map tissue events, cell traffic and identification of protein and chemical mediators, we identified new families of potent bioactive lipid-derived mediators, coined resolvins and protectins in resolving exudates using lipid mediators lipidomics. Each of these pro-resolving mediators controls the duration and magnitude of acute inflammation in vivo with stereospecific sites of action in the pico- to nanogram range. The mapping of these endogenous resolution circuits provides new avenues to harness uncontrolled inflammation and consider the molecular basis of many inflammation-associated diseases [Serhan et al., 2008]. This SLB/IEIIS presentation will overview on our recent advances on the biosynthesis and functions of this novel genus of specialized pro-resolving mediators (SPM). These structurally distinct families of local chemical mediators were originally identified in murine exudates captured during the natural self-limited resolution phase and are also produced by human isolated cells. SPM include 3 families of chemical mediators, resolvins, protectins and the most recent addition, maresins. These are biosynthesized from essential omega- 3 fatty acids (EPA and DHA) and possess potent multi-pronged anti-inflammatory, pro-resolving and anti-microbial actions in murine models of sepsis [Spite et al., 2009]. The actions of SPM proved to be potent, cell type-specific and stereoselective with isolated human cells and in animal diseases. We also used microfluidics to monitor single-cell actions of SPM in tandem to LC-MS-MS lipidomics within $1 \mu \mathrm{l}^{3}$ volume $<5 \mathrm{~min}$ from whole blood. Endogenous formation of resolvins and protectins and their organ-protective roles were confirmed by others and extended into clinical trial. For example, SPM have potent actions and are formed in murine ischemic renal injury [Hassan and Gronert, 2009] in obesity-in- duced insulin resistance and liver disease [Gonzalez-Periz et al., 2009] and in reducing pain [Xu et al., 2010]. Identification of endogenous SPM biosynthesized during acute inflammation-resolution indicates that the resolution of acute inflammation is an active programmed process. These findings change the $>200$-yearold concept that resolution of inflammation is a passive process. Taken together they indicate that natural resolution pathways may underlie many prevalent diseases associated with uncontrolled inflammation and open the potential for resolution-based pharmacology.

The author acknowledges support of NIH grants DE019938, NS067686, GM038765 and DK07448.

\section{References}

Merched A, Ko K, Gotlinger KH, Serhan CN, Chan L: Atherosclerosis: evidence for impairment of resolution of vascular inflammation governed by specific lipid mediators. FASEB J 2008;22:3595-3606.

Schwab JM, Chiang N, Arita M, Serhan CN: Resolvin E1 and protectin D1 activate inflammationresolution programmes. Nature 2007;447:869874.

Serhan CN: Resolution phases of inflammation: novel endogenous antiinflammatory and pro-resolving lipid mediators and pathways. Annu Rev Immunol 2007;25:101-137.

Serhan CN, Chiang N, Van Dyke TE: Resolving inflammation: dual antiinflammatory and pro-resolution lipid mediators. Nat Rev Immunol 2008;8:349-361 (issue cover).

Spite M, Norling LV, Summers L, Yang R, Cooper D, Petasis NA, Flower RJ, Perretti M, Serhan CN: Resolvin D2 is a potent regulator of leukocytes and controls microbial sepsis. Nature 2009;461:1287-1291.

Xu Z-Z, Zhang L, Liu T, Park J-Y, Berta T, Yang R, Serhan CN, Ji R-R: Resolvins attenuate inflammatory pain via central and peripheral actions. Nat Med 2010;16:592-597.

\section{KARGER}

(c) 2010 S. Karger AG, Basel

Fax +41613061234 E-Mail karger@karger.ch www.karger.com 


\section{Journal of \\ Nutrigenetics \\ Nutrigenomics}

\section{Challenges in Nutrigenomics and Nutrigenetics}

\author{
P002 \\ Ghrelin and Adiponectin are Associated with \\ HDL-Cholesterol Levels in Obesity \\ Independently of Insulin Resistance and \\ Inflammation \\ Juan Patricio Nogueira1, Marie Maraninchi', \\ Sophie Béliard², Alain Nicolay³, Réne Valéro ${ }^{4,1}$ \\ ${ }^{1}$ UMR INRA 1260/University of Méditerranée, Faculty of \\ Medicine, La Timone-Marseille, France, ${ }^{2}$ UMR INSERM \\ 939, La Pitié Hospital-Paris, France, ${ }^{3}$ Department of \\ Biochemistry, University of Méditerranée, Sainte \\ Marguerite Hospital Hospital-Marseille, France, \\ ${ }^{4}$ Department of Nutrition, Metabolic diseases, \\ Endocrinology, University of Mediteranée, La Timone \\ Hospital-Marseille, France
}

Objective: To investigate the association between the plasma HDL-c levels and the plasma hormonal profiles (insulin, adipocytokines and ghrelin) in subsets of class II and III obesity patients.

Design and Methods: Fasting plasma levels of glucose, total cholesterol, LDL-cholesterol, HDL-cholesterol, triglycerides, free fatty acids, apoproteins AI, B-100, B-48, C-II, C-III, insulin, CRP, adipocytokines, ghrelin, as well as body composition and resting energy expenditure (REE) were measured in 3 subsets of obese patients: 21 metabolically healthy obese (MHO), 17 metabolically abnormal obese (MAO) and 21 isolated low HDL-cholesterol obese patients (LHO) compared to 21 healthy lean control subjects.

Results: Insulin resistance increased gradually from the MHO to the LHO and the MAO patients $(\mathrm{P}<0.05$ between MHO and MAO and between LHO and MOA for HOMA-IR). In multiple regression analysis, adiponectin was positively associated with HDL-c levels in the MAO group $(\mathrm{P}=0.011)$ and ghrelin was positively associated with HDL-c levels in the LHO group $(\mathrm{P}=0.011)$.

Conclusions: Our study shows that, in obesity, ghrelin and adiponectin could contribute to the HDL-c level independently of insulin resistance and inflammation.

\section{P003}

\section{Minor Components of Mediterranean Diet Reduce Colon Cancer Cell Growth}

Juan J. Moreno, Marisol Cabral, Raquel Martín-Venegas

University of Barcelona, Spain

Colorectal cancer is the second leading cause of cancer-related deaths and epidemiological data suggested that dietary modification could reduce the risk of colorectal cancer by as much as $90 \%$. Owing

\section{KARGER \\ (C) 2010 S. Karger AG, Basel}

Fax +41613061234

E-Mail karger@karger.ch

www.karger.com
Accessible online at: www.karger.com/jnn 
to its high content of fruits, vegetables, fiber, fish and olive oil, the Mediterranean diet is healthy and has many protective effects, prevents cancer, especially of the colon and breast. Olive oil and wine contain several minor components such as tyrosol, $\beta^{2}$-sitosterol and resveratrol that could be involved, at least in part, in these effects. The aim of this study was to determine the action of these compounds on cell cycle and cell growth of human colon cancer cells (Caco-2). Cell growth was determined by microscopic assay using ethidium bromide/acridine orange staining in preconfluent Caco-2 cell cultures in presence of $10 \%$ fetal bovine serum, and cell cycle analysis was performed by flow cytometry. Our results show that tyrosol (1-100 $\mu \mathrm{M})$ and resveratrol $(1-100 \mu \mathrm{M})$ inhibited Caco-2 cell proliferation in a concentration dependent manner whereas $\beta^{2}$-sitosterol $(1-100 \mu \mathrm{M})$ did not effect. Moreover, these polyphenols were able to arrest Caco-2 cells in $\mathrm{S}$ phase. These effects can be correlated with the antioxidant capacity of these compounds. Interestingly, we can observe a synergistic effect between tyrosol and resveratrol which can explain their regulation on cancer cell proliferation at physiological concentrations reached after the consumption of a Mediterranean diet. This study was supported by grant BFU2007-61727/BFI (Ministerio de Ciencia y Tecnología)

\section{P004 \\ Effect of Jacaranda Seed Oil Extrac, Rich in Jacaric Acid, on the PPRE Promoter Activity in Transfected Cells}

\author{
Jonatan Miranda1, Arrate Lasa1, Itziar Churruca1, \\ Alfredo Fernández-Quintela1, Cristina García², \\ Josune Ayo ${ }^{2}$, Reanud Dentin ${ }^{3}$, María del Puy Portillo ${ }^{1}$ \\ ${ }^{1}$ University of the Basque Country-Vitoria Gasteiz, Spain, \\ ${ }^{2}$ Unidad de Investigación Alimentaria AZTI-Tecnalia, \\ ${ }^{3}$ Département d'Endocrinologie, Métabolisme et Cancer. \\ Institut Cochin INSERM U567 CNRS 8104, Paris, France
}

Introduction: The group leaded by Bassagagna-Riera state that plant-derived conjugated triene fatty acids contain some chemical signatures consistently found in most naturally occurring agonists of PPARs (peroxisome Proliferator-Activated Receptor). Published data concerning this issue only refers to punicic acid (cis-9, trans-11, cis13 CLNA) and -eleostearic acid (cis-9, trans-11, trans-13 CLNA). The purpose of the present study was to analyze the effects of jacaranda seed oil extract, rich in jacaric acid (cis-8, trans-10, cis-12 CLNA), on the PPRE (PPAR Response Element) promoter activity in transfected cells.

Methods: HEK cells (Human Kidney Embrionic cells) -293 were transfected with the plasmid under study (PGL2-PPRE-Luc) and with the internal control (galactosidase). Cells were divided into three experimental groups: cells in basal state, cells with over-expression of PPAR and cells with over-expression of PPAR. After 24 hours cells were treated with jacaranda oil at different doses during 18 hours. At this point luciferase and -galactosidase activity was measured.

Results: Treatment (18 hours) with jacaranda oil at different doses $(1,5,10,25,50$ and $100 \mathrm{M}$ of jacarid acid) did not modify luciferase activity of cells at basal state, or cells with PPAR/PPAR receptors over-expressed.
Conclusions: In contrast to other CLNA isomers such as punicic and eleostearic acids, jacaranda seed oil extract, rich in jacaric acid, does not activate PPAR and PPAR receptors.

This study has been supported by Gobierno Vasco: Foodbask 2007 and GIC07/120-IT-265-07.

\section{P005 \\ Bisulfite-Sequencing: A Resolver for Nutri- Epigenetics}

Ning Li, Jun Wang

BGI Shenzhen -Shenzhen, China

Nutrition is closely related to development, health and aging, previous studies presented that different nutrition impacts on development as honey bees with identical genotype but dramatically different phenotype. Thus, Epigenetic modifications like DNA methylation were thought to be key factor involved in this development process. The introduction of high-throughput sequencing with improved accuracy and throughput has greatly enhanced the ability to characterize epigenetic regulations of nutri-epigenetics. And bisulfite-sequencing is the golden standard for DNA methylation. In our study, we generated a single-base-resolution human DNA methylation profile in peripheral blood mononuclear cells (PBMC). Using whole-genome bisulfite sequencing, we report the essentially complete $(92.62 \%)$ methylome of human peripheral blood mononuclear cells (PBMC) which constitute an important source for clinical blood tests worldwide. Analysis of the PBMC methylome revealed a rich landscape of epigenomic data for 20 distinct features including regulatory, proteincoding, RNA gene coding, non-coding and repeat sequences. Integration of our methylome data with the previously determined genome sequence of the same Asian individual analysed here, enabled a first assessment of allele-specific methylation (ASM) differences between the two haploid methylomes of any individual. Using a conservative cut-off $(\mathrm{p}<0.001)$, we identified 599 haploid differentially methylated regions (hDMRs) covering 287 genes. Of these, 76 genes had hDMRs within $2 \mathrm{~kb}$ of their transcriptional start sites of which $>80 \%$ displayed allele-specific expression (ASE) alter random testing using TA clone sequencing of the same PBMC sample. The study performed herein demonstrates a paradigm for large-scale epigenomics studies, thus provided an effective methods for nutri-epigenetics. 
P006

\section{Nutritional Genomics: SNPs Selection for Disease-Causing Genes Considering Protein Biophysical Properties}

Marta González-Castejón ${ }^{1}$, Francisco Marín², Cristina Soler², Isabel Espinosa-Salinas',

Ana Ramírez de Molina', Guillermo Reglero², Arantxa Rodríguez-Casado ${ }^{1}$

${ }^{1}$ Institute IMDEA Food-Madrid, Spain, ${ }^{2}$ Universidad

Autónoma de Madrid, Spain

Disease susceptibility and genetic profile relationship implicates many environmental factors like nutrition. Some diet-regulated genes reduce or increase chronic disease risks and Nutritional Genomics focus on the identification of genetic variations provoking physiological responses to diet and their influence on features of health and disease. Single nucleotide polymorphisms are common variants defining the genotype and used to establish genotypic/phenotypic relationships. Prioritize the SNPs altering the risk of diseases is needed considering tag and functional factors to manage the SNPs selection problem. The study here presented considers both functional significance and clarifying tagging, applied to analyze the consequences of non-synonymous SNPs. Non synonymous SNPs result in a change of amino acid sequence of corresponding protein. Despite considerable advances, correlation between genes and biophysical properties of associated proteins like structure and protein interactions has not yet been explored extensively. Still the polymorphisms effects beyond the gene level are crucial because most cellular processes occur through the proteins interactions and some disease-causing polymorphisms behave changing protein structure. Our approach combines structural and functional characteristics of amino acid substitutions responsible for phenotypic nsSNPs effects. Protein structure modelling and sequence analysis, domain annotation and web resource are used to providing information on disease/gene relationships at the molecular level including protein stability, structure and sequence-based features. Additionally, evaluation of solvent accessibility is done showing that buried residues are less likely to vary and their mutations are more likely to cause disease. The study has been applied to a set of genes involved in the etiology of cholesterol for identification and characterization of pathogenic cholesterol nsSNPs. We consider their significance in the selected genes found in patients at high risk for cholesterol and predict which of them may result in differential response to dietary treatments and encourage to the development of personalized diet and targeted functional ingredients design.

\section{P007}

\section{Caseinophosphopeptides Exert Partial Protection Against $\mathrm{H}_{2} \mathrm{O}_{2}$-Induced Oxidative Stress in Caco-2 Cells}

María José García-Nebot, Antonio Cilla, Amparo Alegría, Reyes Barbera

Faculty of Pharmacy University of Valencia-Burjassot, Spain

Introduction: Caseinophosphopeptides (CPPs) are phosphorylated peptides derived from casein, and are able to sequester prooxidant metals and scavenge free radicals. These properties imply that CPPs can be used as functional food ingredients. On the other hand, oxidative stress (OS) is caused by an accumulation of reactive species (ROS), and has been related to the pathogenesis of inflammatory diseases. The present study was designed to compare the biological effects of a pool of CPPs with/without $\mathrm{H}_{2} \mathrm{O}_{2}$-induced oxidative stress in Caco- 2 cells.

Materials and Methods: A pool of CPPs was obtained by simulated gastrointestinal digestion from casein and selective precipitation. Caco-2 cells were preincubated (24 h) with CPPs (1, 2 and $3 \mathrm{mg}$ / $\mathrm{ml}$ ) and then treated with or without $5 \mathrm{mM} \mathrm{H}_{2} \mathrm{O}_{2}$ for $2 \mathrm{~h}$. Under these conditions, lipid peroxidation (MDA), intracellular accumulation of ROS, mitochondrial membrane potential (MMP) changes, and the effects on cell cycle progression were determined.

Results and Discussion: $\mathrm{H}_{2} \mathrm{O}_{2}$ treatment significantly increased lipid per oxidation vs. control cells or cells incubated with CPPs, while CPPs $+\mathrm{H}_{2} \mathrm{O}_{2}$ prevented this increase - reaching values similar to control cells. A significant increase in ROS and decrease in MMP were observed after $\mathrm{H}_{2} \mathrm{O}_{2}$ treatment vs. control cells or cells incubated with CPPs, whereas treatment with CPPs $+\mathrm{H}_{2} \mathrm{O}_{2}$ did not improve both parameters vs. $\mathrm{H}_{2} \mathrm{O}_{2}$-treated cells. A significant reduction in cell proportions in $\mathrm{G} 1$ and $\mathrm{G} 2 / \mathrm{M}$ phases, together with a significant increase in sub-G1 phase, were observed in cells treated with $\mathrm{H}_{2} \mathrm{O}_{2}$. After OS, incubation of cells with the three doses of CPPs assayed led to the recovery of control levels.

Conclusion: CPPs exert only site-specific protection against OS, diminishing lipid per oxidation and maintaining a cell cycle pattern similar to that of control cells. However, they failed to exert protection at mitochondrial level. This fact suggests that CPPs are unlikely to be considered general cytoprotective agents. 
P008

Identification of a Metabolic Gene

Expression Profile for the Analysis of Lipid Metabolism Alterations on NutritionalRelated Diseases

\section{Ma Isabel Espinosa Salinas', Margarita González-Vallinas', Arantxa Rodríguez-Casado1, Ricardo Ramos², Susana Molina', Ana Ramírez de Molina ${ }^{1}$ \\ ${ }^{1}$ Imdea Food-Madrid, Spain, ${ }^{2}$ Genomic Unit Scientific Park of Madrid, Spain}

Introduction: Dietary prevention or mitigation of major chronic diseases such as obesity, diabetes, cardiovascular disorders or cancer, may benefit strongly from the knowledge of the human genome and the consequently emergence and application of genomics. A common event for these diseases is the alteration of the lipid metabolism and closely-related signalling pathways, pointing at the relevance of the study of the differential metabolic status of the population who develop these diseases. In addition, it is well-known the influence of determined nutrients in lipid metabolism, suggesting an essential role for nutrient-gene interactions in this regard.

Methods: In the present work we have been focused on the establishment of a system for the determination of the expression profile of genes that codify for enzymes of the lipid metabolism with the final aim of stratifying the population based on a metabolic-related gene expression signature, with special emphasis on the identification of new nutritional markers for cardiovascular pathologies, obesity or cancer. Once established the pathways, enzymes, gene variants and probes which will provide a proper global view of the lipid metabolism gene expression profile, the latest technology will be used for relative quantification of gene expression, genotyping and allelic discrimination of different populations.

Results: 120 genes encoding for key enzymes of lipid metabolism pathways and their corresponding interconnections have been selected to constitute the Metabolic Gene Expression Profile. This metabolic-related gene expression signature includes genes involved in the absorption and transport of nutrients, lipid catabolism, biosynthesis and beta-oxidation of fatty acids, ketogenesis, phospholipid metabolism, biosynthesis of bile acids and peroxisomal lipid metabolism.

Conclusions: This study will provide the genetic basis for understanding the lipid metabolism alterations with relevance for the development or/and progression of nutritional-related diseases, and therefore might contribute to the prevention, mitigation or improvement of the management of patients with these diseases.

\section{P009}

Variants in Transferrin Gene Affect Iron Metabolism and Response to an Iron Supplemented Food in Menstruating Women

Ruth Blanco-Rojo', Ana María Pérez-Granados ${ }^{1}$, Ana María López-Parra², Carlos Baeza², Stefania Bertoncini2,3, Eduardo Arroyo-Pardo ${ }^{2}$ Pilar Vaquero ${ }^{1}$

${ }^{1}$ Institute of Food Science and Technology and NutritionMadrid, Spain, ${ }^{2}$ Faculty of Medicine, Complutense University of Madrid, ${ }^{3}$ Anthropology Unit, University of Pisa, Italy

Introduction: Genetic and nutritional factors are involved in iron deficiency. Variants in the transferrin gene explained $\sim 40 \%$ of genetic variation of transferrin levels.

Objectives: To know the association between SNP rs3811647 in the transferring gene and iron status in menstruating women and the influence of variants of this SNP on iron biomarkers changes in response to an iron supplemented food.

Methods and Materials: Menstruating women $(n=284)$ were selected. Inclusion criteria: caucasian women, 18-45 years, nonsmoker, non-pregnant, non-breastfeeding. Exclusion criteria: amenorrhea, menopause or any known health problems likely to influence iron status. Genotyping of SNP rs3811647 was carried out by minisequencing.

Hematocrit, mean corpuscular volume, haemoglobin, serum ferritin and serum transferrin were determined. A subgroup of 122 volunteers (ferritin $<40 \mathrm{ng} / \mathrm{mL}$, haemoglobin $\geq 11 \mathrm{~g} / \mathrm{dL}$ ) participated in a randomized double-blind placebo-controlled study of 16-weeks of duration. A placebo or an iron-enriched food were given to the subgroups $\mathrm{P}(\mathrm{n}=58)$ and $\mathrm{E}(\mathrm{n}=64)$, respectively. Data were analysed by correlation and ANOVA tests.

Results: The SNP rs3811647 was related with serum transferrin $(\mathrm{p}<0.001)$. Serum transferring was significantly higher in AA $(342.2$ $\pm 67.3 \mathrm{mg} / \mathrm{dL})$ than $\mathrm{AG}(314.6 \pm 51.6 \mathrm{mg} / \mathrm{dL})$ and $\mathrm{GG}$ women $(292.2$ $\pm 48.6 \mathrm{mg} / \mathrm{dL})(\mathrm{p}<0.001)$. The $\mathrm{E}$ subgroup presented markedly decrease in serum transferrin, indicating increase in iron status, from week 4 to the end of the assay and the differences between $\mathrm{P}$ and $\mathrm{E}$ were significant at weeks $4,8,12$ and $16(p<0.01)$. However, there were no significant differences between $\mathrm{P}$ and $\mathrm{E}$ women presenting the AA genotype.

Conclusion: The SNP rs3811647 is related with serum transferrin levels in menstruating women. Presence of variant AA in rs 3811647 is associated with lower recovery of iron status after consumption of iron supplemented food.

Supported by Project Ref. AGL2009-11437 R Blanco-Rojo was supported by CSIC and European Social Fund (JAE-predoc grant). 


\section{P010}

\section{Vitamin A Deficiency Modulates Hepatic Hepcidin and Gut Ferroportin mRNA Levels}

Sandra Fernandes Arruda1, Douglas dos Santos Moreira², Fernanda Ribeiro Rosa ${ }^{2}$, Fabiani Lage Rodrigues Beal', Silvana Reigota Naves de Araujo², Egle Machado de Almeida Siqueira ${ }^{4}$

'Department of Nutrition, Faculty of Health ScienceBrasilia, Brazil, ${ }^{2}$ Human Nutrition Graduation Program, Health Science, ${ }^{3}$ Health Sciences Graduation Program, Health Science, ${ }^{4}$ Biological Sciences Institute, Department of Cell, Brazil

We previously observed that vitamin A deficiency induces iron retention and increase lipid per oxidation in tissues, suggesting that vitamin A deficiency disturbs iron homeostasis. This study evaluated the effect of vitamin A deficiency on expression of deciding, DMT-1 and ferroportin mRNA, and on iron status, in the liver and gut of rats. Forty-two male Wistar rats were treated with a Control diet (CT; AIN93G; 4,000 UI/kg) or a Vitamin A deficient diet (VA; AIN-93G without any source of vitamin A). After 0, 15, 30 and 45 days of treatment, the rats were killed by cervical dislocation, and liver and gut were excised for determination of mRNA levels of hepcidin in liver and DMT-1 and ferroportin in gut, by real time RT-PCR. Tissue iron concentration was determined by atomic emission spectrometer (ICPAES). Analysis of variance was used to compare the groups, with $p<$ 0.05 as a significant level. Vitamin A deficient rats showed a deep reduction in hepatic retinol concentration at 15 days of depletion $(\mathrm{p}=$ 0.0007), reaching levels under the detection limit at 30 days. Vitamin A deficient rats showed an increase in hepatic hepcidin mRNA levels $(p=0.029)$, and in gut ferroportin mRNA levels $(p=0.0017)$, however no difference was observed in gut DMT-1 mRNA levels. Iron gut levels observed in the control group changed during all period of treatment, while in VA group it reduced at 15 and 30 days of treatment in relation to the basal level ( $p=0.0021$ and 0.006 , respectively), reaching the same levels of the control group at 45 days. In conclusion, vitamin A deficiency seems to up regulate hepcidin expression and down regulates gut ferroportin with no effect on gut DMT-1 expression. Vitamin A seems to also influence the iron bioavailability, decreasing gut iron content.

\section{P011}

Vitamin A Deficiency Promotes Spleen Iron Overload by Increasing Hepatic Hepcidin and Decreasing Spleen Ferroportin and DMT-1 mRNA Levels

\author{
Egle Machado De Almeida Siqueira', \\ Fabiani Lage Rodrigues Beal, Gustavo Antonio Teixeira \\ Chaves ${ }^{1}$ Luciano Fernandes de Oliveira ${ }^{3}$, \\ Fernando Fortes de Valencia ${ }^{1}$, Sandra Fernandes Arruda ${ }^{4}$ \\ ${ }^{1}$ Biological Sciences Institute, Department of Cell-Brasilia, \\ Brazil, ${ }^{2}$ Health Sciences Graduation Program, Health \\ Science, ${ }^{3}$ Human Nutrition Graduation Program, Health \\ Science, ${ }^{4}$ Department of Nutrition, Faculty of Health \\ Science, Brazil
}

The relationship between vitamin A and iron status has been widely reported, however the exact mechanism involved in this interaction has not been well characterized. The present study investigated the effect of vitamin A deficiency on transcripts of hepcidin, DMT-1 and ferroportin, and on iron status, in the liver and spleen of rats. Forty-two male Wistar rats were treated with a Control diet (CT; AIN93G) or a Vitamin A deficient diet (VA; AIN-93G without any source of vitamin A). After 0, 15, 30 and 45 days of treatment, the rats were killed, liver and spleen were excised for mRNA levels determination of hepcidin in liver and DMT-1 and ferroportin in spleen, by real time RT-PCR. Tissue iron concentration was determined by atomic emission spectrometer (ICP-AES). Analysis of variance was used to compare the groups, $\mathrm{p}<0.05$. After 15 days of vitamin A depletion, the rats showed a significant reduction in hepatic retinol concentration ( $p$ $=0.0007$ ), at 30 days, hepatic retinol was not detected in VA group, and at this period, the vitamin A deficient rats showed an increase in spleen iron concentration $(\mathrm{p}=0.0046)$, while no difference was observed in the liver iron levels between the two groups. Hepatic hepcidin mRNA levels increased significantly in VA group at 30 days of treatment $(p=0.029)$, and no difference was observed on ferroportin mRNA expression, during all treatment period. At 30 days of treatment spleen ferroportin and DMT-1 mRNA levels decreased significantly in VA group ( $\mathrm{p}=0.046$ and 0.015 , respectively). In conclusion, at 30 days of treatment, the vitamin A deficiency unregulated hepcidin mRNA expression in liver, resulting in iron spleen accumulation, probably by the downregulation of ferroportin and DMT-1 mRNA expression in spleen, once these proteins participate in iron export from endossome and splenic cells, respectively. 


\section{P012}

\section{Mediterranean Diet Reduces Senescence Associated Stress in Endothelial Cells}

\author{
Carmen Marin ${ }^{1}$, Rafael Ramirez², Pablo Pérez-Martinez', \\ Julia Carracedo², Javier Delgado-Lista', Ana Merino, \\ Antonio García-Rios ${ }^{1}$, Purificación Gómez', \\ Francisco Pérez-Jiménez ${ }^{1}$, José López-Miranda ${ }^{1}$ \\ ${ }^{1}$ Reina Sofia University Hospital, Lipids and \\ Atherosclerosis Research Unit, University of Cordoba, \\ Spain, ${ }^{2}$ Research Unit, Reina Sofia University Hospital, \\ Instituto de Investigación Biomédica de Cordoba, Spain
}

Senescence of endothelial cells induced either by aging or accelerated pathological conditions might play an important role in the development and progression of atherosclerosis. Studies suggest that high-fat diet induces premature endothelial senescence, reflecting a novel mechanism for diet-induced atherosclerosis. Our aim was to study the effects of the oxidative stress induced by type of dietary fat on cellular senescence.

Methods: 20 elderly subjects consumed three diets, each for four weeks: a saturated fatty acid diet (SFA), a low-fat and high-carbohydrate diet (CHO n-3) and a Mediterranean diet (Med diet) enriched in monounsaturated fatty acid (MUFA) following a randomized crossover design. For each diet, we investigated intracellular reactive oxidative species (ROS), apoptosis and telomere length in HUVEC (human umbilical vein endothelial cell) incubated with serum of each patients.

Results: Med Diet induced lower intracellular ROS levels than SFA and CHO-n3 diet ( $\mathrm{p}=0.013$ and $\mathrm{p}=0.005$, respectively), lower apoptosis levels compared to SFA and CHO-n3 diet $(\mathrm{p}=0.014$ and $p=0.006$, respectively) and lower percentage of cells with short telomeres versus SFA and CHO-n3 diet $(p=0.020$ and $p=0.004$, respectively). In addition, we observed a higher ROS levels after intake of CHO-n3 diet versus SFA diet $(\mathrm{p}=0.012)$. After the incubation of HUVEC with TNF- $f \alpha$, the Med Diet showed lower intracellular ROS levels compared to baseline (0.006) and SFA and CHO-n3 diet ( $\mathrm{p}=0.001$, in both case), lower apoptosis levels versus baseline $(\mathrm{p}=0.007)$ and SFA and CHO-n3 diet $(\mathrm{p}=0.001$, in both case), as well as lower percentage of cells with short telomeres compared to baseline $(\mathrm{p}=0.004)$ and SFA and CHO-n3 diet $(\mathrm{p}=0.004$ and $\mathrm{p}=0.002$ respectively).

Conclusions: The intake of med diet modulates oxidative stress in endothelial cells.

Consequently, the med diet protects these cells from oxidative stress preventing cell senescence and improving survival of endothelial cells.

\section{P013 \\ H63D Mutation and Its Relationship with Deficiency Anaemia Through the Levels of Serum Transferrin}

Carlos Baeza', Ana María López-Parra', Ruth Blanco-Rojo², Ana María Pérez-Granados², Stefania Bertoncini ${ }^{3}$, Eduardo Arroyo-Pardo', M. Pilar Vaquero ${ }^{2}$

${ }^{1}$ Department of Toxicology and Health Legislation, Faculty of Medicine, Complutense University of Madrid, Spain, ${ }^{2}$ Institute of Food Science, Technology and Nutrition Spanish National Research Council, CSIC, ${ }^{3}$ Department of Biology, Anthropology Unit, Pisa, Italy

Introduction: Serum transferrin (TF) is a biomarker employed to assess iron status. Describing genetic factors such as SNPs, which could explain a proportion of its variation, may help us to understand the general genetic basis of iron related disorders which may lead to iron overdose. One of the variants of the SNP rs1799945, the H63D mutation (allele G), has been held as one of the modifiers of the severity of hemochromatosis, and may as well influence iron related parameters.

Objective: To assess the association between the SNP rs 1799945 (H63D) and the levels of TF.

Methods and Materials: Women $(n=284)$ were selected according to the following criteria. Inclusion criteria: caucasian women, 18-45 years, non-smoker, non-pregnant, non-breastfeeding. Exclusion criteria: amenorrhea, menopause or any known health problems likely to influence iron status. Genotyping of SNP rs1799945 was carried out by minisequencing. The following parameters of iron status were determined: hematocrite, mean corpuscular volume, hemoglobin, ferritin and transferrin. The association was assessed by a regression analysis, assuming a codominant effect model for the SNP variants or, in other words, assuming an additive effect for each copy of an allele.

Results: A significant association was found between the levels of serum transferrin and the variants of SNP rs1799945 (H63D), in our sample $(\mathrm{p}<0.029)$. Individuals genotyped as $\mathrm{C} / \mathrm{C}$ showed higher levels of TF $(313.11 \pm 4.19 \mathrm{mg} / \mathrm{dL})$, than those genotyped as $\mathrm{C} / \mathrm{G}$ $(298.43 \pm 5.58 \mathrm{mg} / \mathrm{dL})$ and than those individuals $\mathrm{G} / \mathrm{G}(282.81 \pm 9.56$ $\mathrm{mg} / \mathrm{dL})$.

Conclusion: The SNP rs1799945 is significantly associated with the levels of TF. Each copy of G allele slightly improves iron status in women predisposed to iron deficiency anaemia, therefore presence of the H63D mutation may be considered as a modifier of the severity of iron deficiency anaemia.

Financed by project AGL2009-11437 and a fellowship from CSIC and European Social Fund (JAE-predoc). 
P014

\section{Bitter Taste Genes Polymorphisms and Longevity in an Elderly Italian Population}

Roberto Barale1, Daniele Campa², Paolina Crocco², Maura Carrai ${ }^{1}$, Giuseppina Rose ${ }^{3}$, Federica Canzian ${ }^{3}$, Francesco De Rango ${ }^{3}$, Giuseppe Passarino ${ }^{3}$

${ }^{1}$ Dipartimento di Biologia, Università di Pisa, Italy, ${ }^{2}$ German Cancer Research Center-Heidelberg, Germany,

${ }^{3}$ Dipartimento di Biologia Cellulare Università Della

Calabria-Rende, Italy

Individual taste responsiveness elicited in the mouth by food may play a significant role on food preference. There are indications that this factor may affect dietary attitude, and related health consequences, of individuals throughout their lifespan. Moreover, recent research indicates that the role played by receptors present in lingual taste buds are marginal when compared to the role exercised by the same receptors in the membrane of sensory cells of the neuroendocrine system in the gastrointestinal (GI) by regulating nutrients digestion and absorption. They are particularly important for the control of food intake and digestion by regulating also insulin secretion, small intestine motility and gall bladder contraction. In humans, taste receptors for sweet, umami and bitter are encoded by two gene families (TAS1R and TAS2R) which are highly polymorphic with several alleles with reduced or null functional activity. DNA of 600 elderly (60-100 years old), provided by the Research Unit of University of Calabria, was submitted to "tagging SNPs" analysis of the two chromosome regions comprehending the 25 bitter taste genes. Preliminary results show a significant association between three polymorphisms of two genes encoding receptors for bitter taste perception (TAS2R16, TAS2R46) with longevity, cholesterol and the body mass index (BMI), respectively. These preliminary data strongly suggest that bitter tasting compounds, diet related, play a significant role in the relationship between diet and health and that taste genetic polymorphism can significantly account for interindividual differences in health and longevity.

\section{P015 \\ Impact of Solid Waste on The Genome of Cattle}

\section{Hamid Najeh, Mostafa Kandil \\ Faculty of Science University Chouaib Doukkali-El Jadida, Morocco}

Domestic species can be used to study the effects of toxic substances present in the environment. The contamination level to which animals are exposed can be correlated to the concentration of contaminants in their tissues. The principal aim of this work was to know the background micronucleus frequency of peripheral blood lymphocytes $\mathrm{T}$ in Bovines exposed to garbage (handled originates from industrial, medical, and household) in El Jadida, Morocco. We used the micronucleus $(\mathrm{MN})$ test in cytochalasin B blocked binucleated cells (BNC) to assess the possible induced genetic effects. The cellular proliferation index (PI) was also calculated. Blood samples were collected from bovines exposed to garbage dumps, and peripheral blood cultures were made according to standard methodology. The results showed a significant increase of micronucleated cells in the lymphocytes of the exposed animals compared to the control. This induction was accompanied by a significant inhibition of proliferate index in the lymphocytes of the exposed animals. The exposure to solid garbage was associated with increase genetics damage. The application of the micronucleus test seems to be a suitable method to predict the genotoxic risk. This Obvious genetic risk related to solid garbage exposition show clearly a need of educational programs for the stockbreeders in order to decrease genotoxic risks.

Keywords: Micronuclei; Cell Proliferation; Food contamination; Garbadge.

P016
Reduction of Tissue Factor Activity, Prote
and mRNA Expression in Response to
Polyphenols in Human Aortic Endothelia
Cells Exposed to Pathophysiological Stim
as Tumor Necrosis Factor- $\boldsymbol{\alpha}$
Sara Fernández-Castillejo, Úrsula Catalán, Laia Pons-
Llecha, Rosa Sola
Universitat Rovira i Virgili-Reus, Spain

Introduction: Inflammation, the key component in the pathogenesis of atherosclerosis, promotes thrombosis by the induction of tumor necrosis factor-alpha (TNF- $\alpha)$. Accumulation of tissue factor (TF) in atherosclerotic plaques is thought to play a critical role in determining plaque thrombogenicity. The regulation of TF by polyphenols as resveratrol is predominantly at the level of transcription, mRNA stabilization, and the subsequent expression of the TF protein. However, the amount of TF protein production in vascular cells does not always correlate with TF activity caused by different factors. The aim of the study is to investigate the effects of resveratrol on TF activity and on its gene and protein expression, in human aortic endothelial cells (HAEC) exposed to pathophysiological stimuli such as TNF- $\alpha$.

Methods and Materials: HAEC monolayers were incubated with resveratrol $(5,10,25,50$ and $100 \mu \mathrm{M})$ for $16 \mathrm{~h}$ and then stimulated with TNF- $\alpha(10 \mathrm{ng} / \mathrm{mL})$ over $6 \mathrm{~h}$. Resveratrol cytotoxicity was determined by lactate dehydrogenase (LDH) activity in culture medium. TF activity, protein and mRNA assessments were determined in cell lysates.

Results: Cytotoxicity of resveratrol was examined in HAEC, and no effect was observed. Resveratrol significantly $(p<0.05)$ reduced, in a dose dependent manner, TF activity, protein and mRNA. At maximal dose tested $(100 \mu \mathrm{M})$ resveratrol reduced TF activity by $95 \%$, TF protein expression by $90 \%$, and TF mRNA expression by $60 \%$. At 20 $\mu \mathrm{M}$, resveratrol inhibited the induction of TF activity by $50 \%$.

Conclusions: Our data suggest a strong association between TF activity and its protein concentration while a weaker association is observed between TF activity and its TF mRNA expression on HAEC exposed to resveratrol with equivalent concentrations obtained in human plasma provided by dietary supplementation.

J Nutrigenet Nutrigenomics 2010;3:49-124 75 


\section{P017}

\section{Anthocyanin-Rich Model Foods and their Role in Cardioprotection and Obesity}

\author{
Katia Petroni', Roberto Pilu², Valentina Calvenzani ${ }^{1}$,
}

Chiara Tonelli', Marie-Claire Toufektsian³,

Michel de Lorgeril ${ }^{3}$, Lucilla Titta4, Marco Giorgio ${ }^{4}$

${ }^{1}$ Dipartimento di Scienze Biomolecolari e Biotecnologie,

Università degli di Milano, Italy, ${ }^{2}$ Dipartimento di

Produzione Vegetale, Università degli Studi di Milano, Italy,

${ }^{3}$ Nutrition, Vieillisement et Maladie Cardiovasculaires,

Université Joseph Fourier-Grenoble, France, ${ }^{4}$ Dipartimento

di Oncologia Sperimentale, Istituto Europeo Oncologico-

Milano, Italy

Being commonly found in fruits and vegetables, flavonoids and anthocyanins are widely distributed in the human diet. Epidemiological studies suggested that regular consumption of flavonoid-rich foods or beverages is associated with decreased risk of chronic degenerative diseases. Maize isogenic lines with high levels of hydroxycinnamic acids, flavonols, phlobaphenes and anthocyanins were developed by generating suitable allelic combinations of the MYB and bHLH regulatory gene families controlling activation of the flavonoid biosynthetic pathway in maize. The aim was to provide dietary supplements to test the impact of specific flavonoids in whole foods on cardiovascular and age-related degenerative diseases using animal model systems. These studies demonstrated that in rats fed anthocyanin-rich blue maize the amount of cardiac tissue that was damaged following ischemic conditions was reduced by approximately $30 \%$ compared to rats fed anthocyanin-free maize. Cardio protection was associated with increased myocardial glutathione levels, suggesting that dietary anthocyanins modulate cardiac antioxidant defenses. Furthermore, we investigated the effect of blood and common orange juice on fat accumulation in mice fed standard or high fat diet. Results revealed that providing blood orange juice, but not common orange juice, as a substitute of water, reduced significantly body weight gain and fat accumulation, thus impairing the high-fat induced obesity. The gene expression profile of fat tissue from mice eating high-fat diet and drinking blood orange juice strikingly resembled that of mice fed standard diet.

\section{P018 \\ Downregulation of the Hepatic SIRT6 Gene Expression in Metabolic Syndrome: Association with Oxidative Stress}

\section{Ana B. Crujeiras, Omar Al-Massadi, Luisa M. Seoane, María Pardo, María Amil-Diz, Cecilia Castelao, \\ Felipe F. Casanueva \\ Instituto de Investigación Sanitaria Hospital Clínico- Santiago de Compostela, Spain}

Aim: SIRT6 plays an important role in preventing aging phenotypes and tumor development. Metabolic syndrome is associated with impaired liver fibrogenesis leading to hepatocarcinogenesis, in which oxidative stress appears to be involved. Therefore, the aim of this study was to evaluate the hepatic gene expression of SIRT6 and its relationship with genes implicated in the oxidative stress network in Zucker rats, an animal model for the metabolic syndrome.

Methods: The liver was isolated from male Zucker (fa/fa; $\mathrm{n}=9$ ) rats and their littermates $(+/ ? ; n=10)$ of 22 weeks of aged and the RNA was obtain to perform the gene expression of SIRT6, SIRT1 and Glutahione-S-transferase (GST-M2), an antioxidant enzyme, by qRTPCR. The animals were housed under controlled conditions of temperature, humidity and light illumination and they were allowed access to water and food ad libitum throughout the experimental period according to standard protocols and recommendations for the proper care and use of laboratory animals.

Results: Interestingly, hepatic SIRT6 was down regulated in male Zucker rats (fold-change: $0.73(0.71-0.75))$ compared with their littermates, similar to SIRT1 $(0.53(0.50-0.56)$ and GST-M2 $(0.67$ (0.65-0.70). Hepatic SIRT6 gene expression was directly associated with hepatic SIRT1 ( $\mathrm{r}=0.59 ; \mathrm{p}=0.007)$ and, especially in obese group, with GST-M2 ( $\mathrm{r}=0.70 ; \mathrm{p}=0.037)$ gene expression. Moreover, higher body weight $(\mathrm{r}=-0.53 ; \mathrm{p}=0.019)$ and fat mass $(\mathrm{r}=-0.55 ; \mathrm{p}=0.014)$ was correlated with down regulation of hepatic SIRT6.

Conclusion: These results provide a new insight about the potential use of SIRT6 as new biomarker for oxidative stress and in relation to obesity related-comorbidities. Further studies are needed to evaluate the role of this less known sirtuin in the obesity therapy.

\section{P019}

\section{A Diet Enriched in Polyphenols and Polyunsaturated Fatty Acids, LMN Diet, Affects Aging and the Phenotype of a Mouse Model of Alzheimer Disease, Tg2576}

\author{
Laura Fernández', Gemma Comes², Irene Bolea', \\ Patricia Murtra ${ }^{1}$, Bartolomé Ramirez ${ }^{3}$, Neus Anglés ${ }^{3}$, \\ Merce Boada4, Juan Hidalgo², Rosa María Escorihuela ${ }^{5}$, \\ Mercedes Unzeta ${ }^{1}$ \\ ${ }^{1}$ Institute of Neurosciences and Department of \\ Biochemistry and Molecular Biology, Faculty of Medicine, \\ Autonomous University of Barcelona-Bellaterra, Spain, \\ ${ }^{2}$ Institute of Neurosciences and Department of Cellular \\ Biology and Immunology, Animal Phisiology Unit, \\ Faculty of Sciences Autonomous University of \\ Barcelona-Bellaterra, Spain, ${ }^{3}$ La Morella Nuts SA, Reus- \\ Tarragona, Spain, ${ }^{4}$ Fundació ACE, Institut Catalá de \\ Neurociencies Aplicades-Barcelona, Spain, ${ }^{5}$ Medical \\ Psychology Unit, Department of Psychiatry and Forensic \\ Medicine, Autonomous University of Barcelona-Ballaterra, \\ Spain
}

Introduction: Normal aging and Alzheimer disease (AD) are accompanied by declines in motor and cognitive performance. AD a neurodegenerative disease characterized also by the deposition of extracellular amyloid plaques in cortex and hippocampus. Our previous results ruled out the properties of $* \mathrm{LMN}$ diet, a natural diet enriched in polyphenols and polyunsaturated fatty acids, as a modulator of neurogenesis in adult mouse. The aim of this work is study both prevention and beneficial LMN effect in aging and $\mathrm{AD}$. 
Methods and Materials: Two groups of transgenic mouse AD model, Tg2576, and 129S1/SvImJ (WT) mice were fed with a standard Harlan 2014 control and LMN diets during 3 and 5 months. All mice were weighed continuously every week to evaluate the possible LMN diet effect on the body weight. Animals were sacrificed, plasma was recovered for ELISA determinations and brains were processed for immunohistological or western-blot studies. A $\beta$ content and GFAP expression were determined. Morris Water Maze, were assessed to 18 months age male's mice fed control or LMN diet.

Results: Regarding the body weight at the time of killing, there are not significant differences or suggestive trends towards a dietspecific effect on weight gain. Results from behavioral test reveals that, while control $\operatorname{Tg} 2576$ mice exhibited a spatial learning impairment, relative to wild type (WT) mice, LMN-fed mice exhibited spatial memory retention comparable to four months mice. Thus its effect inducing cell proliferation in hippocampus, could explain the beneficial effect on behaviour. LMN diet affect also the $A \beta$ content in Tg2576, a decreasing tendency was observed in hippocampus; such decreasing effect of $\mathrm{LMN}$ diet on $\mathrm{AB}$ levels was significant for the $\mathrm{AB}$ 1-40 AB 1-42 and plasma levels.

Conclusions: These data suggest the beneficial effect of LMN diet on aging and neurodegenerative diseases.

\section{P020 \\ A Nutrigenomic Frame to Identify Time- Resolving Physiological Responses of Hepatic Genes in Diet-Induced Obesity Mice Utilizing Microarray-Based Gene Expression}

Seon-Min Jeon', Gyeong-Min Do', Eun-Young Kwon'1, YunYoung Cho', Hae-Jin Park', Su-Kyung Shin', CheolGoo Hur ${ }^{2}$, Myung-Sook Choi ${ }^{1}$

${ }^{1}$ Kyungpook National University-Daegu, Republic of Korea, ${ }^{2}$ Korea Research Institute of Bioscience and

Biotechnology, Republic of Korea

Introduction: Chronic intake of high-fat diet isa major cause of insulin resistance and obesity. We investigated to identify the alteration of gene expression by long-term consumption of high-fat diet.

Methods and Materials: To compare the hepatic gene transcription within same dietary group and between normal diet (ND) group and high-fat diet (HFD) group, C57BL/6J mice were sacrificed at $2,4,6,7,8,12,16,20$ and 24 weeks in sequence, and hepatic DNA microarray was performed.

Results: We selected the HFD-responsive down- or up-regulated genes over 2-fold and the genes within FDR q-val<0.1 based on the ND group at 0 wk. Changes of gene transcripts within each ND and HFD group were the largest at time-point week 6, week 8 and week 24. Steroid synthesis, and insulin and PPAR signaling pathwayrelated genes were long-term down-regulated, and cancer pathwayrelated genes were long-term up-regulated in responsive to HFD. Genes related to the fatty acid metabolism and pancreatic cancer were classified in the early down-regulated HFD-responsive gene network, and genes related to the T-cell receptor signaling pathway, cell adhesion molecules and glycolysis/gluconeogenesis were selected in the early up-regulated HFD-responsive gene network. Hepatic genes that were down-regulated after $12 \mathrm{wks}$ were related to the insulin signal- ing pathway and steroid biosynthesis, and those that were up-regulated after 12 wks were related to the colorectal and pancreatic cancer.

Conclusions: We suggest that long-term intake of HFD induces the alteration of gene expression by affecting various biological pathway including lipid metabolism, insulin signaling pathway, inflammatory metabolism.

\section{P021}

\section{Polyphenols from Red Propolis Suppresses Angiogenesis Through Promoting Hypoxia- Inducible Factor -1alpha (HIF-1) Degradation Dependent of Von Hippel-Lindau}

\author{
Julio Beltrame Daleprane1, Thomas Prates Ong", \\ Masaharu Ikegaki², Heidi Menrad', Theresa Gueis', \\ Tobias Schmid ${ }^{3}$, Nathalie Dehne ${ }^{3}$, Bernhard Brüne ${ }^{3}$, \\ Dulcinéia Saes Parra Abdalla ${ }^{1}$ \\ ${ }^{1}$ Department of Food and Experimental Nutrition, Fac-Säo \\ Paulo, Brazil, ${ }^{2}$ Department of Pharmacy, Federal University \\ of Alfe, Brazil, ${ }^{3}$ Institute of Biochemistry I, Faculty of \\ Medicine, Brazil
}

Polyphenols from Red Propolis (PRP) is reported to might modulate angiogenic process therefore we investigated the effect of PRP in angiogencic process. Viability of endothelial cell (EC) was measured by MTT assay. EC sprouting from murine aortic rings, formation of new blood vessels in the chorio-allantoic chiken embryo (CAM), differentiation of stem cell in CD-31 positive cells and migrations of EC cell were used as angiogenesis models. To describe the mechanism that PRP modulate angiogenic process we used RT-qPCR and Western blot. Maximum concentration of PRP tolerated by EC was $10 \mathrm{~g} / \mathrm{mL}$, PRP reduced sprouting of EC in aortic rings, formation of new blood vessels in CAM and inhibit the differentiation of stem cell in to CD-31 positive cell, in 40, 65 and 55\% respectively, when compared to control. We found that PRP inhibited VEGF mRNA and protein expression in EC. As hypoxia-inducible factor 1 (HIF-1) is one major transcription factor regulating VEGF induction, PRP reduced protein but not mRNA expression of HIF-1. The half-life of HIF-1 protein was 38 and $58 \mathrm{~min}$ in presence and absence of PRP, respectively, suggesting an enhance protein degradation in the presence of PRP. PRP decreased HIF-1 protein expression in RCC4 cell which had reconstituted von Hippel-Lindau (pVHL) cells but not in RCC4 cell lacking pVHL. Additionally, migration of EC was inhibited by PRP to similar amount as a knockdown for HIF-1 EC's. Our results demonstrated that PRP inhibits VEGF expression by increasing HIF-1 protein degradation through $\mathrm{pVHL}$ dependent mechanism. Indicating that loss of HIF-1 expression induced by PRP is followed by reduced angiogenic responses in EC. This finding provides new insight into molecular mechanism underlying the anti-angiogenic action of PRP. 
P022

\section{Lipoic Acid Increases Hepatic Mitochondrial Biogenesis in a Diet-Induced Obesity Rat Model}

\author{
M. Pilar Valdecantos ${ }^{1}$, Patricia Pérez-Matute ${ }^{2}$, Pedro Prieto- \\ Hontoria', María J. Moreno-Aliaga1, J. Alfredo Martínez' \\ ${ }^{1}$ University of Navarra-Pamplona, Spain, ${ }^{2} \mathrm{HIV}$ and \\ Associated Metabolic Alterations Unit Infectious Disease \\ Area Center for Biomedical Research of La Rioja (CIBIR)- \\ Logroño, Spain
}

Introduction: Mitochondrial dysfunction can lead to the development of several metabolic diseases such as obesity. Devising therapeutics to manage mitochondrial function and/or biogenesis is an attractive strategy for preventing these disorders. PGC1 and SIRT1 have been characterised as key regulators of mitochondrial biogenesis. In this context, lipoic acid (LA) is a natural compound with beneficial effects on fuel metabolism, obesity and mitochondrial oxidative stress. The purpose of the present study was to determine the effects of supplementation of a high fat diet with LA on hepatic mitochondrial biogenesis focusing on its actions on several genes involved in mitochondrial function.

Methods: Male Wistar rats were assigned in two dietary groups fed with a standard diet $(\mathrm{n}=10)$ or a high fat diet (HFD). HFD animals were also distributed in two subgroups: OB group: fed with a HFD ad libitum $(n=10)$ and OLIP group $(n=10)$ : where the diet was supplemented with LA (0.25 g/100 g of diet). SIRT1, SIRT3, PGC1 NRF1 and COXII mRNA levels in liver were determined by Real time-PCR. The mitochondrial copy number was determined by the ratio between mRNA levels of a specific mitochondrial gene (COXII) and a nuclear housekeeping gene.

Results: Mitochondrial copy number was significantly decreased by the ingestion of a high fat $\operatorname{diet}(\mathrm{P}<0.05)$, whereas LA supplementation was able to reverse this inhibitory effect ( $\mathrm{P}<0.0001$ vs $\mathrm{OB})$. Furthermore, a significant decrease in the mRNA levels of several genes involved in mitochondrial biogenesis such as SIRT1, SIRT3, PGC1 and NRF1 were observed in the obese group. However, LA treatment was able to significantly reverse and even increase the expression of these genes $(\mathrm{P}<0.05-\mathrm{P}<0.01$ vs $\mathrm{OB})$.

Conclusions: These results demonstrate that LA is able to increase the expression of certain genes involved in mitochondrial function and to improve the hepatic mitochondrial biogenesis despite of a high fat diet.

\section{P023}

Apolipoprotein A5 Gene Variation Interacts with Dietary Fat Intake to Modulate Obesity and Triglyceride Levels in a Mediterranean Population (Garaulet Study)

\author{
Carmen Sánchez Moreno ${ }^{1}$, José María Ordovás ${ }^{2,3}$, \\ Juan Carlos Baraza1, Juan José Alburquerque-Béjar', \\ Mari Paz Carrasco-Benso', Jesús López-Mínguez', \\ Marta Garaulet ${ }^{1,2}$ \\ ${ }^{1}$ University of Murcia, Spain, ${ }^{2}$ Jean Mayer US Department \\ of Agriculture Human Nutrition Research Center on Aging, \\ Tufts University School of Medicine-Boston, MA, ${ }^{3}$ The \\ Department of Epidemiology and Population Genetics \\ Centro Nacional Investigación Cardiovasculares (CNIC)- \\ Madrid, Spain
}

Introduction: APOA5 is the most recently identified apolipoprotein regulating plasma triglycerides (TG) and the least well characterized in terms of functional mechanism. It is possible that alleles associated with increased disease risk may be silenced in the presence of a Mediterranean Diet.

Methods and Materials: We have investigated the interactions between a polymorphism at the APOA5 gene $(-1131 \mathrm{~T}>\mathrm{C})$ and dietary fat that may modulate the levels of TG-rich lipoproteins and anthropometric measures in overweight and obese subjects. We recruited 1500 subjects from a Mediterranean population (BMI: $25-40 \mathrm{~kg} / \mathrm{m}^{2}$ ) of 20-65 y. who attended outpatient obesity clinics in Spain.

Results: We found an association between the APOA5-1131T $>C$ SNP and TG and VLDL levels that were higher in carriers of the minor allele than in non-carriers. Consistent with previous findings we found a gene-diet interaction between the $-1131 \mathrm{~T}>\mathrm{C}$ polymorphism and fat intake (g) with relation to obesity. Subjects homozygous for the $1131 \mathrm{~T}$ major allele demonstrated a positive association between fat intake and obesity. Conversely, in subjects carrying the APOA51131C minor allele, higher fat intakes were not associated with higher BMI. We also found strong gene-diet interactions between the SNP and fat intake $(\mathrm{g})$ for both lipids TG and VLDL $(\mathrm{P}<0.001)$.

Conclusions: In conclusion we have replicated previous genediet interactions between APOA5-1131T $>$ C polymorphism and fat intake for obesity traits, and detected an interaction for plasma TG and VLDL. The minor allele appears to be protective for both the accumulation of fat, and for higher plasma TG in people consuming higher levels of dietary fat. 
P024

Dietary Macronutrient Composition Is Related to High-Density Lipoprotein Particle Size and Subspecies Distribution Independent of Genetic Effects: A Monozygotic Twin Pair Study

\author{
Leonie-Helen Bogl', Marianna Maranghi',3, Aila Rissanen ${ }^{4}$, \\ Jaakko Kaprio ${ }^{1,5,6}$, Marja-Riitta Taskinen ${ }^{7}$, \\ Kirsi Hannele Pietilinen ${ }^{1,2,4}$ \\ ${ }^{1}$ The Finnish Twin Cohort Study, Department of Public \\ Health, University of Helsinki, Finland, ${ }^{2}$ Department of \\ Medicine, Division of Diabetes, Helsinki University Central \\ Hospital-Helsinki, Finland, ${ }^{3}$ Department of Internal \\ Medicine and Medical Specialties, Sapienza University- \\ Roma, Italy, ${ }^{4}$ Obesity Research Unit, Department of \\ Psychiatry-Helsinki, Finland, ${ }^{5}$ Department of Mental Health \\ and Substance Abuse Services, National Institute for \\ Health and Welfare-Helsimki, Finland, ${ }^{6}$ Institute for \\ Molecular Medicine-Helsinki, Finland, ${ }^{7}$ Department of \\ Medicine, Division of Cardiology, Helsinki University \\ Central Hospital-Helsinki, Finland
}

Introduction: HDL-C levels, size and distribution are influenced by genetic and environmental factors. Recently, the composition of the diet has been related to HDL-C levels and patterns. We aimed to examine the relationship between macronutrient intake and HDL particle size and subspecies distribution independently of genetic effects, by studying monozygotic (MZ) twins.

Methods: Twenty-four healthy pairs of MZ twins aged 23-33 years were identified from two longitudinal population-based studies, FinnTwin 16 and FinnTwin12. Total energy and macronutrient intake were assessed with three-day food diaries, whereas exercise was evaluated by a physical activity index. Mean HDL size and subspecies distribution were determined by non-denaturing gradient gel electrophoresis. Results: In multivariate nutrient density models adjusted for confounding variables, replacing 5\% of energy from carbohydrates with polyunsaturated fatty acids (PUFAs) resulted in a significantly higher percentage of large HDL2b ( $\mathrm{a} \pm \mathrm{SE}: 15.37 \pm 6.49, \mathrm{p}<0.05$ ) particles. Substitution of the same energy amount with protein was associated respectively with higher and lower percentages of HDL2a $(\hat{a} \pm$ SE: $3.73 \pm 1.37, p<0.05)$ and HDL3b $(\hat{a} \pm$ SE: $-3.48 \pm 1.25$, $\mathrm{p}<0.05$ ) particles, showing a tendency towards bigger particle diameter. Partial correlation analyses, adjusted for sex and BMI, between HDL subspecies and PUFAs were more consistent for omega-3 (n-3) PUFAs. Within each twin pair, mean HDL size was significantly higher in the co-twins with a higher n-3 PUFAs intake. Accordingly, these subjects displayed higher percentages of large HDL2b particles and lower percentages of HDL $3 a$ and HDL $3 b(p<0.05)$ subspecies as compared to those with a lower n-3 PUFAs intake. HDL size and distribution did not differ between co-twins consuming high and low amount of n-6 PUFAs.

Conclusion: Our data suggest that dietary protein and n-3 PUFAs intake are associated with a favorable change in the distribution of HDL subspecies towards larger particles independent of genetic influences.

\section{P025}

Effect of Restructured Meats Containing Sea Spaghetti on Liver Cholesterol $7 \alpha$-hydroxylase Expression in Normal and Hypercholesterolaemic Male Wistar Rats

\author{
Adriana Rita Schultz Moreira', Raúl Olivero David², \\ Laura González Torres ${ }^{1}$, Miguel Vázquez Velasco', \\ Sara Bastida', Juana Bened³, Francisco José Sánchez- \\ Muniz \\ 1Departamento de Nutrición y Bromatología I, Facultad de \\ Farmacia. Universidad Complutense de Madrid, Spain, \\ ${ }^{2}$ Departamento de Nutrición y Bromatología, Facultad de \\ Farmacia Universidad de Alcalá, Spain, ${ }^{3}$ Departamento de \\ Farmacología Facultad de Farmacia, Universidad \\ Complutense de Madrid, Spain
}

Introduction: Restructured meats containing seaweeds are potential functional foods. Sea Spaghetti (Himanthalia elongata) is an excellent nutritional, gastronomic value, and is thought to have antioxidant activity. The aim of this investigation was to identify whether the liver cholesterol $7 \alpha$-hydroxylase expression of normal or hypercholesterolaemic rats was affected by diets including restructured meats containing Sea Spaghetti for a period of 5 weeks.

Materials and Methods: Male Wistar rats with free access to the diet were divided into 4 groups $(n=10)$. (1) Control diet which consisted of $85 \%$ rodent chow (AIN-93M purified rodent diet) and $15 \%$ meat (containing $4 \%$ cellulose-wheat starch mix $(96: 4, \mathrm{w} / \mathrm{w})$ ). (2) Sea Spaghetti diet which consisted of $85 \%$ rodent chow with meat $15 \%$ meat (containing $5 \%$ Sea Spaghetti); (3) Hypercholesterolaemic diet (Control diet but with $2 \%$ cholesterol and $0 \bullet 4 \%$ colic acid); (4) Hypercholesterolaemic- Sea Spaghetti diet (same as the hypercholesterolaemic diet but with 15\% meat (containing $5 \%$ Sea Spaghetti). Following a 5 week feeding period liver tissues were excised from rat, mRNA was extracted and cholesterol $7 \alpha$-hydroxylase expression was quantified using Reverse Transcription Polymerase Chain Reaction (RT-PCR).

Results: The expression of liver cholesterol $7 \alpha$-hydroxylase was increased 44-60\% compared with control in normal and hypercholesterolaemic rats eating normal or Sea Spaghetti-supplemented diets. Cholesterol $7 \alpha$-hydroxylase expression levels were consistently (13$29 \%$ ) increased in animals consuming diets supplemented with Sea Spaghetti.

Conclusions: Rises in liver cholesterol $7 \alpha$-hydroxylase expression in rats consuming meat Sea Spaghetti diets can be explained by the sterols contents in Sea Spaghetti, which seem to play important physiological roles in fat absorption and cholesterol metabolism. 


\section{P026 \\ Microarray Analysis of Human Umbilical Vein Endothelial Cells (HUVEC) Treated with the w-3 Fatty Acid Docosahexaenoic Acid (DHA) Highlights New Anti-Atherosclerotic and Anti-Angiogenic Properties for Fish and Fish Oil}

\author{
Egeria Scoditti', Marika Massaro', \\ María Annunziata Carluccio', Rosanna Martinelli'2,3, \\ Tonia Buonomo², Giuseppe Martines ${ }^{4}$, Carlo Storelli, \\ Raffaele De Caterina ${ }^{6}$ \\ ${ }^{1}$ C.N.R. Institute of Clinical Physiology, Pisa and-Lecce, \\ Italy, ${ }^{2}$ CEINGE Biotecnologie Avanzate-Naples, Italy, \\ ${ }^{3}$ Department of Biochemistry and Medical Biotecnologies, \\ Federico II University of Naples, Italy, ${ }^{4}$ Città di Lecce \\ Hospital-Lecce, Italy, ${ }^{5}$ Department of Biological and \\ Environmental Science and Technology (Disteba), \\ University of Salento-Lecce, Italy, ${ }^{6 " G}$. d'Annunzio" \\ University-Chieti, Italy
}

Background and Aim: High intakes of $\omega$-3 fatty acids have been associated with systemic anti-inflammatory effects and cardiovascular protection, but the molecular basis responsible for these effects remains incompletely defined. Using a DNA microarray technology we investigated the early gene expression profile of human vascular endothelium conditioned by DHA under proinflammatory conditions.

Materials and Methods: HUVEC were treated with $50 \mu \mathrm{mol} / \mathrm{L}$ DHA for 48 hours and then stimulated with $5 \mathrm{ng} / \mathrm{mL}$ IL-1 $\beta$ for 3 hours. Total RNA was extracted, and qualitatively and quantitatively analyzed with a NanoDrop Spectrophotometer and an Agilent Bioanalyzer before RNA labeling and purification. Gene expression profile was performed with an Agilent Whole Human Genome Oligo Microarray covering 41000 unique genes and transcripts. Slides were scanned with the Agilent's scanner and images processed using Agilent Feature Extraction software. The raw data were further processed with the GeneSpring ${ }^{\circledR} 10$ software and differentially expressed RNA identified using Benjamini and Hochberg False Discovery Rate with a p-value $<0.05$. Functional and network analyses were identified by the Ingenuity Pathways version 8.0 Analysis.

Results and Conclusions: IL-1 stimulation significantly changed the expression of 1474 genes: 815 resulted down-regulated while 659 resulted up-regulated. Focusing on the 659 IL-1-upregulated genes, we observed that DHA significantly attenuated the expression of 88 such genes. The application of the Ingenuity pathway analysis software allowed us to pinpoint immunological-, inflammatory and atherogenic-related pathways as the most affected. In particular, we identified new target molecules involved in atherosclerosis, including tubulin beta polypeptide[TUBB] $2 \mathrm{~A}$ and phosphodiesterase $[\mathrm{PDE}] 5 \mathrm{~A}$; and in angiogenesis, including transforming growth factor [TGF] $\beta 2$ and chemokine (C-X-C motif) ligand 10 . In conclusion, DHA widely affects endothelial gene expression; the identification of novel genes susceptible to DHA will certainly improve our understanding of mechanisms by which fish oils may prevent or attenuate human chronic diseases including atherosclerosis.

\section{P027 \\ Unraveling the Paradoxes of ATM Resensitized Dynamics in LNCaP Cell Line Via Epigallocatechin-3-gallate (EGCG)}

Ammad Farooqi

The University of Lahore, Pakistan

Epigallocatechin-3-gallate (EGCG) is a major ingredient of green tea (GT) presumably holds a potential to prevent pathogenomics. Prostate cancer aggressiveness is triggered by fusion transcripts formed because of genomic instability induced by juxtapositioning of two genes. An abolished ATM dynamics is incapable of safeguarding integrity of DNA. In agreement with this assumption ATM and DNA PK were impaired in LNCaP cell line to confirm a tight interaction of ATM and DNA PK with the expression profile of TMPRSS2-ERG. Abolished ATM proved instrumental to expression of the fusion transcript. Similarly blunting of DNA PK down regulated the expression of the fusion transcript giving a notion that it is involved in the chromosomal translocation. LNCaP cell lines were analyzed for the effect of EGCG on the expression profile of TMPRSS2-ERG. In this particular unprecedented study treatment of the LNCaP cell line with EGCG recapitulated ATM expression and activity and down regulated the fusion transcript generation. These results underscore the therapeutic effect of EGCG in attenuating the exacerbation of the disease.

\section{Cardiovascular and Lipids}

\section{P028 \\ Anti-Atherogenic and Anti-Angiogenic Activity of Polyphenols from Propolis}

\author{
Julio Daleprane1, Masaharu Ikegaki², Thomas Ong ${ }^{1}$, \\ Luis Salazar ${ }^{3}$, Dulcineia Abdalla \\ ${ }^{1}$ Universidade de São Paulo, Brazil, ²Universidade Federal \\ de Alfenas, Brazil, ${ }^{3}$ Universidad de la Frontera, Brazil
}

Introduction: Effects of polyphenols from different sources of propolis on atherosclerotic lesions, inflammatory and angiogenic factors were investigated in LDL receptor gene (LDLr-/-) knockout mice.

Methods and Materials: The animals received a cholesterolenriched diet to induce atherosclerosis. The animals were divided into 3 groups, receiving polyphenols from green, red or brown propolis ( $250 \mathrm{mg} / \mathrm{Kg} /$ day) by gavage during 4 weeks. The athermanous lesions were analyzed for gene expression of inflammatory and angiogenic factors by qRT-PCR.

Results and Conclusions: The polyphenols improved lipid profile, decreased atherosclerotic lesion area and expression of inflammatory factors. Furthermore, the expression of the pro-angiogenic factors FGF, PDGF, VEGF, PECAM and MMP-9 was down 
regulated while the metalloproteinase inhibitor TIMP-1 gene was up regulated by polyphenols from all types of propolis. In contrast, only the polyphenols from red propolis induced dowregulation of CD36 expression and up regulation of the antioxidant enzyme HO-1 and TIMP-1 genes compared to polyphenols of the other two types of propolis. Concluding, polyphenols from propolis are atheroprotective through mechanisms including modulation of inflammatory and angiogenic factors.

Financial Support: Foundation for Research Support of the State of São Paulo, FAPESP, grants to D.S.P.A. (08/53756-7) and Ph.D. scholarship to J.B.D. (08/53755-0).

\section{P029 \\ The Interplay Between Folate Intake, MTHFR C677T Polymorphism, and Lipid Metabolism in the Elderly}

\author{
Agata Chmurzynska1, Anna Malinowska', \\ Jolanta Twardowska-Rajewska², Jan Gawecki \\ ${ }^{1}$ Poznan University of Life Sciences-Helsinki, Finland, \\ ${ }^{2}$ Adam Mickiewicz University, Gerontology Laboratory, \\ Poznan, Poland
}

Hyperhomocysteinemia may be caused by reduced folate intake and impaired folate metabolism, which is dependent on the polymorphism of genes encoding folate and homocysteine metabolism-related enzymes, including methylenetetrahydrofolate reductase (MTHFR). The aim of the study was to evaluate the influence of MTHFR C677T polymorphism on the level of serum folate in the elderly. Moreover, the impact of folic acid supplementation on the lipid metabolism parameters was analyzed. 142 Poles aged 60 years were supplemented with $0.4 \mathrm{mg} / \mathrm{d}$ of folic acid for 8 weeks. The serum folate level, and the total, LDL, and HDL cholesterol and triacylglycerol levels were determined. The daily folate intake was estimated with the use of a food frequency questionnaire. The folate intake did not affect the serum folate level. Neither folate intake nor serum folate concentration differed across genotypes. Higher total cholesterol levels were observed among TT individuals $(259.458 .6 \mathrm{mg} / \mathrm{dL})$ compared to CC (226.2; $37.9 \mathrm{mg} / \mathrm{dL})$ and CT $(231.6 ; 41.4 \mathrm{mg} / \mathrm{dL})$ individuals. Folic acid treatment affected LDL cholesterol, which increased by $15.5 \mathrm{mg} /$ dL among TT homozygotes only. MTHFR C677T polymorphism does not affect the serum folate level, but may be associated with lipid metabolism in the elderly.

\section{P030 \\ Effects of a n-3 Polyunsaturated Fatty Acidssupplementation with or without Fishgelatin Supplementation on Gene Expression in Peripheral Blood Mononuclear Cells in Obese Insulin-Resistant Subjects}

Iwona Rudkowska', Charles Lavigne ${ }^{1,2}$, Hélène Jacques ${ }^{1,3}$, André Marette ${ }^{1,2}$, Marie-Claude Voh/1,3,4

${ }^{1}$ Institute of Nutraceuticals and Functional Foods (INAF) Laval University-Quebec City, Canada, ${ }^{2}$ Quebec Heart and Lung Institute Research Centre, Laval University-Quebec, Canada, ${ }^{3}$ Department of Food Science and Nutrition, Laval University-Quebec, Canada, ${ }^{4}$ Laboratory of Endocrinology and Genomics, Laval University Hospital Research Center, Laval University-Quebec, Canada

It is well-known that $n-3$ polyunsaturated fatty acids (PUFA) supplementation may benefit individuals. The addition of fish proteins may have supplementary beneficial effects to n-3 PUFA. Further, gene expression profiling is thought to be more sensitive to nutritional intervention than the traditional biochemical parameters.

Aim: To investigate gene expression changes in peripheral blood mononuclear cells (PBMCs) following an-3 PUFA and n-3 PUFA plus fish gelatin (FG) supplementation.

Methods: We carried out a transcriptome comparison of 8-week supplementation with n-3 PUFA (1.8 g per day) and n-3 PUFA+FG ( 1.8 g per day $n-3$ PUFA $+25 \%$ of daily protein intake) in PBMCs of 16 obese insulin-resistant subjects. Changes in gene expression levels were assessed by the Human-6 v3 Expression Bead Chips (Illumina).

Results: Erythrocyte n-3 PUFA concentration increased and plasma TG decreased significantly without altering any inflammatory parameters (including C-reactive protein, tumor necrosis factor-alpha, interleukin-6) after both supplementations. N-3 PUFA supplementation changed the expression of 805 genes, whereas n-3 PUFA+FG supplementation altered the expression of only 187 genes. Three genes were commonly changed after both supplementation periods: fatty acid desaturase 1 (FADS1), free fatty acid receptor 3 (FFR3) and ectodyspasin (EDA). Pathway analysis demonstrated increase in gene expression changes via the nuclear receptor peroxisome proliferatorsactivated receptor alpha (PPARA) and the hypoxia-inducible factor (HIF) signaling pathways after both supplementations. In addition, only n-3 PUFA supplementation resulted in a decreased expression of genes involved in nuclear transcription factor kappaB signaling (NF$\mathrm{kB}$ ). N-3 PUFA+FG supplementation resulted in anincreased expression of genes implicated in NF-kB and oxidative stress; yet, the oxidative stress response mediated by nuclear factor (erythroidderived 2)-like 2 (Nrf2) was greater.

Conclusion: Even if biochemical results were comparable after both supplementations, n-3 PUFA and n-3 PUFA+FG supplementation have each a distinct impact on gene expression levels and metabolic pathways.

Funding: AFMnet. 
P031

\section{Effects of PPARS, Dietary Fat Intakes and Gene-Diet Interactions on LDL Peak Particle Diameter}

Annie Bouchard-Mercier ${ }^{1,2}$, Gaston Godin 3 , Benoît Lamarche1, Louis Pérusse 1,4, Marie-Claude Voh/1,2,1

${ }^{1}$ Institute of Nutraceuticals Functional Foods INAF Laval University-Québec, Qc, Canada, '2Laboratory of Endocrinology and Genomics, Laval University Hospital Research Center, ${ }^{3}$ Faculty of Nursing, Laval UniversityQuebec, Qc, Canada, ${ }^{4}$ Division of Kinesiology, Department of Social and Preventive Medicine, Laval University-

Quebec, Qc, Canada

Gene-diet interactions influence different cardiovascular disease (CVD) risk factors.LDL peak particle diameter (LDL-PPD) has been recognized as an emerging risk factor for CVD. The objective is to examine whether gene-diet interaction effects influence LDL-PPD.

Methods: A total of 674 participants aged between 18 and 55 years were recruited to participate into the INFOGENE Study. Nutritional assessment was performed using a validated food frequency questionnaire. LDL-PPD was determined by non denaturing $2-16 \%$ polyacrylamide gradient gel electrophoresis. Peroxisome proliferator-activated receptor (PPAR) gene polymorphisms PPARL162V (rs1800206), PPAR P12A(rs1801282) and PPAR -87T >C (rs2016520) were determined by PCR-RFLP.

Results: Gene-diet interaction effects on LDL-PPD were observed for PPAR L162V polymorphism with saturated fat $(\mathrm{P}=0.0005)$, total dietary fat $(\mathrm{P}=0.006)$, monounsaturated fat $(\mathrm{P}=0.02)$ and omega- 3 fatty acid intakes $(\mathrm{P}=0.03)$. Specifically, among carriers of the PPAR V162, subjects in the third tertile of saturated fat intake had smaller LDL-PPD than those in the first tertile $(254.4 \pm 2.7 \AA$ vs. $256.4 \pm 2.6 \AA$, respectively, $\mathrm{P}=0.007$ ). In contrast, among homozygous for the PPAR L162 allele, subjects in the third saturated fat intake tertile had larger LDL-PPD than those in the first tertile of saturated fat intake $(255.9 \pm 2.7 \AA$ vs. $255.1 \pm 2.6 \AA$ respectively, $\mathrm{P}=0.01)$. Genediet interactions were also found for PPAR P12A polymorphism with saturated fat intake $(\mathrm{P}=0.04)$ and for PPAR $-87 \mathrm{~T}>\mathrm{C}$ with polyunsaturated/saturated fat ratio $(\mathrm{P}=0.03)$.

Conclusions: Overall, carriers of PPAR L162V polymorphism who had higher intakes of saturated fat presented values of LDL-PPD below the threshold value of $255 \AA$ and thus, may be at higher risk of CVD. Gene-diet interaction effects on LDL-PPD were observed with the three PPAR gene polymorphisms. These findings reinforce the importance to consider dietary factors when studying the impact of different polymorphisms on CVD risk factors.

Funding: CIHR (\# OHN 63276)

\section{P032}

Effect of the S19W Polymorphism in the APOA5 Gene on Growth, Insulin Resistance Markers, and Lipoprotein Levels in Normoweight Neonates
Francisco José Sánchez Muniz', Eva Gesteiro², Sara Bastida1, Dolores Corella ${ }^{3}$, José Maria Ordovas ${ }^{4}$, Maria Luisa Guillen ${ }^{3}$
'Departamento de Nutrición y Bromatología I (Nutrición) Facultad de Farmacia Universidad Complutense de Madrid, Spain, ${ }^{2}$ Servicio de Análisis Clínicos. Hospital de Mérida, Spain, ${ }^{3}$ Departamento de Medicina Preventiva y CIBER Fisiopatología de la Obesidad y Nutrición ISCIII Facultad de Medicina Universidad de Valencia, Spain, ${ }^{4}$ Nutrition and Genomics Laboratory Jean Mayer USDA Human Nutrition Research Centre en Aging, TUFT University-Boston, MA, USA

Background: Apolipoprotein A5 (APOA5) is a protein involved in the activation of lipoprotein lipase (LPL) and the metabolism of triglyceride (TG) rich lipoproteins. Lipoprotein lipase (LPL) is a major player in the metabolism of triglyceride (TG) rich lipoproteins and it has been shown that placental LPL activity positively correlates with fat deposition and size in the fetus. LPL is activated by several factors including apolipoprotein A5 (APOA5).

Aim: To examine the association between the common APOA5 S19W polymorphism and neonatal anthropometrical measurements, lipoprotein and hormone concentrations and insulin sensitivity in 58 White normal newborns from the Mérida cohort.

Results: We found that the presence of the $\mathrm{W}$ allele was associated with lower BMI $(\mathrm{P}<0.001)$, ponderal index $(\mathrm{P}<0.001)$, birth weight $(\mathrm{P}<0.01)$, insulin $(\mathrm{P}<0.05)$, insulin/cortisol ratio $(\mathrm{P}<0.05)$ and HOMA-R $(\mathrm{P}<0.05)$ values, and apoB levels $(\mathrm{P}<0.01)$ as compared with S-homozygous.

Conclusion: The APOA5 S19W polymorphism was associated with fetal development as well as glucose and lipoprotein metabolism of normal neonates. Namely, carriers of the $\mathrm{W}$ allele presented lower body weight and plasma insulin and APOB concentrations and lower insulin/cortisol suggesting poorer gestacional nutrition than homozygotes for the common $\mathrm{S}$ allele.

Keywords: Apo A5 gene, Apo B, appropriate for gestational age, fetal growth, HOMA, insulin resistance markers, lipids, neonatos 
P033

\section{Bioactive Compounds Improve Endothelial Dysfunction by Reducing VCAM-1 mRNA Expression and Its Soluble Protein Form in Human Aortic Endothelial Cells}

\author{
Ursula Catalan', Laia Pons', Sara Fernandez¹, \\ Mercedes Heras', Neus Angles², José Morello², \\ Rosa Sola \\ ${ }^{1}$ Unitat de Recerca en Lipids i Arteriosclerosi, CIB-Reus, \\ Spain, ${ }^{2}$ La Morella Nuts S.A., Castellvell del Camp, Spain
}

Introduction: Atherosclerosis can be considered as the clinical endpoint of an inflammatory process and endothelial dysfunction. In endothelial dysfunction, vascular cell adhesion molecule-1 (VCAM1) expression is increased by pro-inflammatory cytokines such as tumor necrosis factor (TNF-) while products that improve endothelial dysfunction have been poorly explored. The aim of the study was to evaluate the effect of alpha-tocopherol (AT) and BAY 11-7082 (BAY), antioxidant and anti-inflammatory molecules, on VCAM mRNA expression and secretion of the protein soluble VCAM-1 form (sVCAM-1) in the human aortic endothelial cells (HAEC), model challenged with TNF-, inducing endothelial dysfunction.

Materials and Methods: VCAM-1 mRNA expression was measured on exposure for $24 \mathrm{~h}$ to BAY $(0.1-0.2 \mu \mathrm{M})$ and TNF- $(1 \mathrm{ng} /$ $\mathrm{mL})$. The sVCAM-1 protein secretion on exposure for $24 \mathrm{~h}$ to BAY $(0.5,1,2$ and $10 \mu \mathrm{M})$ and TNF $(10 \mathrm{ng} / \mathrm{mL})$ were measured. Following AT $(10-150 \mu \mathrm{M})$ incubation for $6 \mathrm{~h}$, then stimulation with TNF; for $24 \mathrm{~h}(10 \mathrm{ng} / \mathrm{mL}$ and $1 \mathrm{ng} / \mathrm{mL}$, respectively) the VCAM- $1 \mathrm{mRNA}$ expression and $\mathrm{sVC}$.

Results: Compared to TNF-alone stimulated cells, BAY reduced VCAM mRNA expression by $30 \%$ and SVCAM-1 protein secretion by $70 \%(\mathrm{P}<0.05)$. AT, at 75 and $150 \mu \mathrm{M}$, decreased by $30 \%$ VCAM mRNA expression and by $20 \%$ sVCAM- 1 protein, compared to TNFalone stimulated cells $(\mathrm{P}<0.05)$.

Conclusions: This study demonstrates that AT and BAY, besides having antioxidant and anti-inflammatory properties, reduced VCAM mRNA expression and SVCAM-1 protein secretion and, as such, reversed endothelial dysfunction induced by TNF in HAECAM-1 protein secretion were determined.

\section{P034}

\section{Human Transcriptome Response After Mediterranean Diet Consumption}

Olga Castañer ${ }^{1,9}$, María-lsabel Covas ${ }^{1,8}$, Dolores Corella², Olga Portolés ${ }^{2,8}$, Ramón Estruch ${ }^{3,8}$, Rosa LamuelaRaventós ${ }^{4,8}$, Valentina Ruiz-Gutiérrez ${ }^{5}$, Jordi SalasSalvadó6,8, Fernando Arós ${ }^{7}$, Montserrat Fitó ${ }^{8}$

${ }^{1}$ Cardiovascular risk and Nutrition Research Group IMIMInstitut de Recerca de I'Hospital del Mar-Barcelona, Spain, ${ }^{2}$ Department of Preventive Medicine and Public Health, University of Valencia, Spain, ${ }^{3}$ Department of Internal Medicine Hospital Clinic, IDIBAPS-Barcelona, Spain, ${ }^{4}$ Department of Nutrition and Bromatology, University of Barcelona, Spain, 5 Instituto de la Grasa, CSIC-Sevilla, Spain, ${ }^{6}$ Human Nutrition Department, School of Medicine, University Rovira I Virgili-Reus, Spain, ${ }^{7}$ Department of cardiology, Hospital Txangorritxu-Vitoria, Spain, ${ }^{8} \mathrm{CIBER}$ de Fisiopatología de la Obesidad y Nutrición, Spain

Introduction: Despite the benefits associated with healthy diets, data on the mechanisms by which these benefits are promoted are scarce. Our aim was to explore the global transcriptomic response of biological pathways related to cardiovascular disease associated with traditional Mediterranean diet (TMD) intervention.

Methods: The PREDIMED study is a large on-going, parallel, multicentre, randomized, controlled trial aimed at assessing the TMD effect on primary cardiovascular prevention. High cardiovascular risk participants were recruited and assigned to one of the following interventions: 1) TMD plus virgin olive oil (VOO); 2) TMD plus mixed nuts; or 3) low-fat diet (control group). In a sub sample of 30 volunteers of the PREDIMED-Barcelona Sur Centre, gene expression changes in peripheral mononuclear cells, after 3 months of intervention, were assessed by microarray analysis (Affymetrix) in which about 30,000 individual human genes were included. Crude and adjusted models for data analyses were performed separately in two different centres. Pearson's correlation coefficients for log2ratio (post-intervention/pre-intervention value) and T-statistics were greater than 0.97 . Gene ontology analyses were performed by Bioingenuity Software on genes with T-statistics $\geq 1.5$ or $\geq-1.5$ after interventions.

Results: Analyses of canonical pathways related with cardiovascular risk highlighted that: 1) MUFA versus PUFA rich diets (MUFA/ PUFA ratio $>3.5$; TMD plus VOO and Low-fat) promoted changes in clusters of genes associated with cytokine and nuclear receptor signaling; and 2) TMD plus VOO promoted changes in blood pressure related pathways. In agreement with this, the greatest decrease in systolic and diastolic blood pressure levels were observed after TMD plus VOO diet.

Conclusions: One of the mechanisms by which MUFA rich diets, and particularly a TMD rich in virgin olive oil, can exert their health benefits is through an enhancement of the global transcriptomic response in pathways related with cardiovascular risk. 
P035

\section{Oleic Acid and Minor Compounds of Virgin Olive Oil Are Involved in the Beneficial Effects of Olive Oil on Plasma Nitric Oxide and Blood Pressure}

\author{
Rosario Casillas ${ }^{1}$, Carolina Storniolo ${ }^{1}$, Jordi Salas², \\ Maribel Covas², Valentina Ruiz-Gutiérrez', \\ Miguel Ángel Martínez', Ramón Estruch², \\ María Teresa Mitjavila', Juan José Moreno \\ ${ }^{1}$ RTIC 06/0045, Instituto de Salud Carlos III, ²CIBER 06/03, \\ Instituto de Salud Carlos III, Spain
}

Circulating free fatty acids (FFAs) are elevated in metabolic syndrome and hypertension and may cause the impairment of endothelial function and are associated with cardiovascular risk factors linked to insulin resistance including hypertension. Endothelium maintains NO production and preserves vascular protection as consequence of vascular relaxation, inhibition of platelet aggregation and adhesion and inhibition of leukocyte adhesion. High FFAs levels contribute to hypertension and cardiovascular disease by impairing NO-dependent relaxation. In this study, we evaluated the effect of oleic and linoleic acids on NO release by endothelial cells in presence of olive oil minor compounds such as tyrosol and sitosterol. These findings are compared with the changes of NO serum levels and blood pressure after 12-month follow-up virgin olive oil (rich in oleic acid and minor compounds) or nuts (rich in linoleic acid) intervention in aged nonsmoking women at high cardiovascular risk recruited into the largescale trial PREDIMED. Results showed that hypertensive patients had low serum NO levels and that olive oil intervention significantly enhanced NO serum levels while reduced blood pressure. Oleic acid $(0-100 \mu \mathrm{mol} / \mathrm{L})$ reduced NO release by ECV304 cells. This effect was more marked with linoleic acid $(0-100 \mu \mathrm{mol} / \mathrm{L})$. Interestingly, the presence of tyrosol $(10-30 \mu \mathrm{M})$ and sitosterol $(10-30 \mu \mathrm{M})$ or both $(30$ $\mu \mathrm{M})$ reverted the impairment of NO concentrations induced by oleic acid and reached NO levels higher than control condition. These findings that can be related with the increase of sera NO levels as well as the reduction of blood pressure in patients that consumed olive oil diet.

\section{P036 \\ Study of the Association of SNP in GALNT2, TRIB1 and CELSR2 with Plasma Lipids in a High Cardiovascular Risk Spanish Population and Its Modulation by the Mediterranean Diet}

\author{
Dolores Corella', Carolina Ortega-Azorin', José V. Sorli', \\ Oscar Coltel/2, Marisa Guillen', Carmen Saiz'1, \\ Paula Carrasco', José I. González', Ramón Estruch³, \\ José María Ordovas ${ }^{4}$ \\ ${ }^{1}$ University of Valencia-Valencia, Spain, ${ }^{2}$ University Jaume \\ I-Castellón, ${ }^{3}$ Hospital Clinic-Barcelona, ${ }^{4}$ Tufts University- \\ Boston, USA
}

Introduction: Genome-wide association (GWA) studies have identified new genetic determinants of plasma lipids. Among them CELSR2/SORT1 (Cadherin, EGF LAG seven-pass G-type receptor 2/ Sortilin 1), TRIB1 (Tribbles homolog 1) and GALNT2 (UDP-Nacetyl-alpha-D-galactosamine: polype ptide $\mathrm{N}$-acetylgalactosaminyltransferase 2), were associated with LDL-C, triglyceridesand HDL-C/ triglycerides, respectively. However, further replication has been modest and none of these studies evaluated the potential modulation by the Mediterranean diet (MEDiet).

Methods: We analyzed the association between rs4846914 in GALNT2, rs17321515 in TRIB1 and rs646776 near CELSR2/SORT1 and plasma lipids as well as their modulations by adherence to the MEDiet in a high cardiovascular risk population (1011 participants in the PREDIMED-Valencia study, aged $67+/-7$ years), that fulfilled at least one of two criteria: type 2 diabetes; 3 or more cardiovascular risk factors. Subjects with triglycerides $>600 \mathrm{mg} / \mathrm{dL}$ were excluded. Adherence to MEDiet was assessed by a 14-item questionnaire. Values of 0 or 1 were assigned, and the greater score, the greater the adherence. Two groups were considered (high and low).

Results: Prevalence of rs 4846914 was $45 \%$ AA, $43 \%$ AG and $12 \%$ GG; rs 17321515 was $31 \%$ AA, $50 \%$ AG and $19 \%$ GG; and rs 646776 were $65 \%$ CC, $32 \%$ CG and $3 \%$ GG. The TRIB 1 rs646776 variant allele was associated with lower triglycerides (137, 126 and $124 \mathrm{mg} / \mathrm{dL}$ for AA, AG and GG, respectively; $\mathrm{P}=0.038$ ) in the whole population. Although a trend to lower LDL-C in CC subjects for the rs646776 as well as a trend to higher trigyclerides in $\mathrm{G}$ allele carriers of the rs 4846914 was observed, we did not detect statistically significant differences for any SNP. Adherence to the MEDiet modulated some of these associations. Thus, a low MEDiet score increased LDL-C concentrations in CC subjects (rs646776), resulting in significant differences between genotypes $(\mathrm{P}=0.035)$.

Conclusions: The rs17321515 in TRIB1 was associated with triglyceride concentrations, whereas rs4846914 and rs646776 were modulated by the MEDiet. 


\section{P037}

\section{Interaction Between VEGF Receptor (VEGFR2) Gene Polymorphism and Dietary Patterns with Blood Glucose and Lipid Levels in Malaysian Chinese Adults}

\author{
Roseline Wai Kuan Yap, Yoshihiro Shidoji, \\ Motofumi Masaki \\ University of Nagasaki, Japan
}

Brief Introduction: Gene-environment interaction studies involving genetic and dietary factors provide new information in the treatment and prevention of chronic diseases. Three common VEGFR2 gene polymorphisms (rs2305948, rs2071559 and rs1870377) have been associated with coronary heart disease and stroke. Dietary pattern approach (a combination of nutrients) is recommended for nutritional epidemiologic studies involving dietary intake with chronic diseases. This study evaluated the effects of VEGFR2 gene polymorphism (rs1870377) and dietary patterns on physical and biochemical risk factors of chronic diseases in 179 Malaysian Chinese adults (75 men and 104 women) aged 30-65 years.

Methods and Materials: Genotyping of VEGFR2 gene polymorphism was performed by real-time PCR method with TaqMan probes. Dietary intake information was obtained using semi-quantitative food frequency questionnaire (FFQ) and factor analysis was applied to identify major dietary patterns. Physical measurements: body mass index (BMI), systolic (SBP) and diastolic blood pressure (DBP) and biochemical parameters: blood glucose, HbA1c and blood lipid profile were also measured. Kruskal Wallis and 2-way ANOVA tests were used for statistical analyses.

Results and conclusions: Two major dietary patterns were extracted: Balanced diet and Meat, rice and noodles diet. Significant differences in total cholesterol and LDL levels were observed in the three genotypes of rs 1870377 and also significant differences in age, BMI, DBP, triglycerides levels, HDL levels and total cholesterol/ HDL ratio among the three categories of the Meat, rice and noodles diet $(p<0.05)$. No significant differences were observed in all the physical measurements for rs 1870377 polymorphism and all parameters in the Balanced diet dietary pattern $(\mathrm{p}>0.05)$. The interaction between VEGFR2 gene polymorphism (rs1870377) and Meat, rice and noodles diet had also significant effect on both blood glucose and lipid levels after adjusting for confounders $(p<0.05)$. The combined effect of genetic and dietary factors is a better predictor in determining the risks of chronic diseases.

\section{P038 \\ The Effect of the 4G/5G Polymorphism on PAI-1act in a Black South African Population Karin Conradie1, Tiny Hoekstra², Marline Pieters ${ }^{1}$ \\ ${ }^{1}$ North-West University-Potchefstroom, South Africa, \\ ${ }^{2}$ University Medical Center- Utrecht, The Netherlands}

Introduction: Increased plasminogen activator inhibitor-1 (PAI1 ) is considered to be a major risk factor for cardiovascular disease.
PAI- levels in black Africans are, however, known to be lower than that of Caucasians. Plasma PAI-1 levels are regulated by various factors including genetic influences such as the $4 \mathrm{G} / 5 \mathrm{G}$ polymorphism. Very little information is available regarding the $4 \mathrm{G} / 5 \mathrm{G}$ polymorphism in black South Africans and if and how it is related to the lower PAI-1 levels observed.

Methods and Materials: PAI-1act was measured in 2000 black South Africans from the PURE study. Apparently healthy men and women were randomly selected from rural and urban areas in the North West Province. The 4G/5G polymorphism was determined by real- time PCR using allele specific primers and probes.

Results: The allele frequencies were: $2.6 \%, 24.9 \%$ and $72.5 \%$ for the $4 \mathrm{G}$ homozygous, heterozygous and the $5 \mathrm{G}$ homozygous subjects, respectively. In Caucasians the distribution differs with the prevalence of the $4 \mathrm{G} / 5 \mathrm{G}$ as the highest, the $4 \mathrm{G} / 4 \mathrm{G}$ intermediate and the $5 \mathrm{G} / 5 \mathrm{G}$ the lowest. The median PAI-1act for the total population was $4.26 \mathrm{U} / \mathrm{ml}(1.27-7.91 \mathrm{U} / \mathrm{ml})$. The median PAI-1act for the different genotypes was: $6.39 \mathrm{U} / \mathrm{ml}(1.04-7.64 \mathrm{U} / \mathrm{ml}), 4.54 \mathrm{U} / \mathrm{ml}(1.72-8.77 \mathrm{U} /$ $\mathrm{ml})$ and $3.97 \mathrm{U} / \mathrm{ml}(2.96-9.70 \mathrm{U} / \mathrm{ml})$ for the $4 \mathrm{G}$ homozygous, heterozygous and the $5 \mathrm{G}$ homozygous subjects, respectively.

Conclusion: The results indicate that the $4 \mathrm{G} / 5 \mathrm{G}$ polymorphism has an influence on the PAI- 1 act. The $5 \mathrm{G} / 5 \mathrm{G}$ polymorphism associated with low PAI-act has a prevalence of $72.5 \%$ in the studied population. The prevalence of this polymorphism is much higher than in Caucasians and this might explain why PAI-1 levels are lower in the black South African population.

\section{P039 \\ The Antioxidant Hydroxytyrosol Suppresses MMP-9 Activity and Expression in Human Monocytes Through PKC $\alpha / \beta I$ and NADPH Oxidase Inhibition - A Mechanism for Plaque Stabilization by an Olive Oil Component of Mediterranean Diets}

\author{
María Annunziata Carluccio ${ }^{1}$, Egeria Scoditti', \\ Marika Massaro1, Giuseppe Martines², Carlo Storelli3, \\ Raffaele De Caterina ${ }^{4}$ \\ ${ }^{1}$ C.N.R. Institute of Clinical Physiology, Pisa and-Lecce, \\ Italy, ${ }^{2}$ Città di Lecce Hospital-Lecce, Italy, ${ }^{3}$ Department of \\ Biological and Environmental Science and Technology \\ (DISTEBA) University of Salento-Lecce, Italy, ${ }^{4 " G}$. \\ d'Annunzio"University and Center of Excellence on Aging- \\ Chieti, Italy
}

Objectives: Mediterranean diets, of which olive oil is an important component, are associated with a low prevalence of cardiovascular diseases. The production of inflammatory mediators and the activation of matrix metalloproteinase(MMP)-9 by macrophages likely contribute to plaque instability leading to acute coronary events. We studied the effects of the olive oil phenolic antioxidant hydroxytyrosol (HT) on MMP-9 activity and expression in human monocytes, and explored underlying mechanisms.

Methods: Human monocytes were treated with 1-50 $\mu \mathrm{mol} / \mathrm{L}$ HT or with inhibitors of PKC, NADPH oxidase or COX isoenzymes for $30 \mathrm{~min}$ before stimulation with $30 \mathrm{nmol} / \mathrm{L}$ phorbol myristate acetate (PMA) for $24 \mathrm{~h}$. Cell supernatants were tested for the release of 
MMP-9, PGE 2 and tissue inhibitors of MP (TIMPs) by ELISA, and MMP-9 activity by zymography. Cell protein extracts were analyzed by Western analysis for COX-2 expression and for membrane translocation of PKCs and the NADPH oxidase p47 $7^{\text {phox }}$ subunit. The activation of the transcription factor Nuclear Factor (NF)- $\kappa$ B was assessed by EMSA.

Results: PMA induced the release of MMP-9 in monocytes through conventional PKC activation. Cell exposure to HT before PMA stimulation reduced MMP-9 activity and expression $\left(\mathrm{IC}_{50}\right.$ for HT of $10 \mu \mathrm{mol} / \mathrm{L} \mathrm{p}<0.01)$ without affecting TIMP-1 release. Correspondingly, HT inhibited PMA-induced $\mathrm{PGE}_{2}$ production (by $54 \pm 7 \%$ ) and COX-2 expression (by $43 \pm 5 \%$ ) without affecting COX1. Inhibition by HT was mediated by the suppression of NF- $\kappa \mathrm{B}$ and the NADPH oxidase $\mathrm{p} 47^{\mathrm{phox}}$ and $\mathrm{PKC} \alpha / \beta 1$ activation.

Conclusions: Our findings show that HT, at concentrations nutritionally achievable, inhibits the expression and release of MMP-9 at least in part through a suppression of COX-2-dependent $\mathrm{PGE}_{2}$ pathways. Such effect occurs through the attenuation of $P K C \alpha / \beta 1$ and NADPH oxidase activation. Overall, such results contribute to explaining the vascular protective effects exerted by olive oil in Mediterranean diets.

\section{Diabetes}

\section{P040 \\ Interleukin-10 Improves Glucose Metabolism by Increasing Expression of Genes Involved in Beta-Oxidation and Mitochondrial Oxphos in the Skeletal Muscle}

\author{
Agussalim Bukhari'1,2, Isao Usui', Shiho Fujisaka', \\ Yukiko Kanatani', Satoko Senda', Yu Yamazaki', \\ Hikari Suzuki', Masaharu Urakaze1, Masashi Konayashi", \\ Kazuyuki Tobe ${ }^{1}$ \\ ${ }^{1}$ The First Department of Internal Medicine, University of \\ Toyama, Japan, ${ }^{2}$ Department of Nutrition, Faculty of \\ Medicine, Hasanuddin University, Makassar, Indonesia
}

Background: Insulin resistance is well established to be associated with obesity-induced chronic low-grade inflammation. In the current study we examined the effects of an anti-inflammatory IL-10 over expression on insulin action in obese and lean mice.

Material \& Method: C57BL/6J mice were fed with high fat to induce insulin resistance. The mice were then injected with either Ad-hIL-10 or control vector. Glucose homeostasis were checked by IP-GTT and ITT. Insulin signaling in the liver, fat tissue and skeletal muscles were examined by western blot analysis. Expression of the gluconegenic \& lipogenic genes in the liver and genes involved in mitochondrial beta-oxidation and OXPHOS in skeletal muscle were analyzed by Real Time (RT) PCR. The number of macrophage accumulation in fat tissue was examined by immunohistochemistry. To evaluate the central effect of IL-10 we performed intracerebroventricular (ICV) infusion of IL-10.
Results: High fat feeding induced insulin resistance as shown by increased blood level of glucose, insulin, and inhibited insulin signaling in the liver, adipose tissue and skeletal muscle. Blood glucose and insulin levels during IP-GTT/ITT were lower in Ad-hIL-10 injected mice. Improved insulin signaling was observed in skeletal muscles and livers of Adh-IL-10-injected mice. Expressions of the antiinflammatory markers, MRC1, YM1, CD163, CD209, and the gluconeogenic genes, G6Pase and PEPCK as well as the lipogenic gene of SREBP1c were lower in the liver of IL-10-overexpressing mice. In addition, increased expression of genes involved in mitochondrial OXPHOS and beta-oxidation were observed in skeletal muscle which is not a direct effect but may in part due to increased STAT3 phosphorylation in the hypothalamus.

Conclusion: Over expression of IL-10 was capable to recover the high fat-induced insulin resistance in obese mice which was associated with enhanced insulin signaling in the liver and skeletal muscle via a direct and central effect.

\section{P041 \\ GSPE Effects on Proliferation and Apoptosis of Pancreatic $\beta$-cells in Genetically Obese Rats}

\author{
Lídia Cedó Giné, Anna Castell-Auví, Victor Pallares, \\ Mayte Blay, Anna Ardevol, Montserrat Pinent \\ Universitat Rovira i Virgili-Tarragona, Spain
}

Introduction: Procyanidins are phenolic compounds from the flavanoids group widely found in foods and drinks of plant origin such as grapes, cocoa, apples, red wine or chocolate. They function as antioxidants and anti-inflammatories and studies done in our research group suggested that grape seed procyanidin extract (GSPE) affects glucose metabolism modulating plasma insulin levels, pointing out at pancreas as a possible target for procyanidins. They have also been reported to modulate proliferation and apoptosis in several tissues. The aim of the present work is to evaluate the effect of GSPE on proliferation and apoptosis of pancreatic $\beta$-cells in a model of genetically obese rats.

Materials and Methods: Female Zucker fa/fa rats were treated with $35 \mathrm{mg} / \mathrm{kg}$ of GSPE for nearly 10 weeks. We also had a group of Zucker fa/fa rats and Zucker lean rats as control groups. At the end of the treatment, the animals were sacrificed and the pancreatic islets were extracted. We counted the number of islets and we analyzed the apoptosis by ELISA (detection of cytoplasmic DNA-histone fragments), and Bcl-2, CyclinD2 and Mki67 expression by RT-PCR. All statistic analysis was performed using SPSS software.

Results: The number of islets isolated from GSPE-treated rats tended to be higher than that of control fa/fa rats. However, GSPE down-regulated the proliferation markers CyclinD2 and Mki67, suggesting that the GSPE-treated rats are not in the same stage of proliferation than control fa/fa rats. GSPE did not modify the induced apoptosis observed in fa/fa rats, although it down-regulated the antiapoptotic marker Bcl-2, pointing out at a role of GSPE in modulating some proteins involved in apoptosis regulation.

Conclusion: In genetically obese rats, GSPE tends to increase the islet number does not modify the apoptosis of fa/fa rats, and it 
modulates the gene expression of proteins involved in regulation of such processes.

\section{P042 \\ High Meat Consumption Is Associated to Type 1 Diabetes}

\author{
Sergio Muntoni', Roberto Mereu', Luigi Atzori², \\ Alessandra Mereu ${ }^{3}$, Sabrina Galassi ${ }^{1}$, Estefanía Corda', \\ Paola Frongia 4 , Paolo Pusceddu 4 , Paolo Contu ${ }^{3}$, \\ Sandro Muntoni ${ }^{2}$ \\ ${ }^{1}$ The ME.DI.CO. Association-Cagliari, Italy, ${ }^{2}$ Department of \\ Toxicology, University of Cagliari, ${ }^{3}$ Department of Public \\ Health, University of Cagliari, ${ }^{4}$ S. Michele Hospital-Cagliari, \\ Italy
}

Introduction: The worldwide variation in type 1 diabetes (T1D) incidence is genetically based, while its increasing incidence is environmentally determined. Among environmental factors, nutrition plays an important role.

Methods and Materials: We carried out a case-control study in 298 children $0-15$ year's old, 145 of which were affected by T1D. The diet of all children and of their mothers during pregnancy and lactation was assessed.

Results: A statistically significant association was found between T1D and the amount of meat consumption during the first two years of life, while no other nutritional factors were associated with children's T1D.

Conclusions: The diabetogenic effect of meat probably rests on its strong insulinogenic response. Since high meat consumption has been shown to be associated also with ischemic heart disease, stroke, various forms of cancer and type 2 diabetes, a global strategy for prevention of no communicable diseases sharing the same risk factors should be anticipated to the very early periods of life through dietary advice, in order to prevent also T1D.

\section{P043}

\section{Impact of the 19 Known Genetic Risk Variants on the Incidence of Diabetes in the Finnish Diabetes Prevention Study}

\author{
Matti Uusitupa', Alena Stanèáková2, Markku Peltonen ${ }^{3}$, \\ Jaakko Tuomilehto 4 , Markku Laakso ${ }^{5}$ \\ ${ }^{1}$ University of Eastern Finland, Institute of Public Health \\ and Clinical Nutrition-Kuopio, Finland, ${ }^{2}$ University of \\ Eastern Finland, Department of Medicine, Institute of \\ Clinical Medicine, ${ }^{3}$ National Institute for Health and \\ Welfare, Department of Health Promotion and Chronic \\ Disease Prevention, ${ }^{4}$ University of Helsinki, Department of \\ Public Health, ${ }^{5}$ University of Eastern Finland, Department \\ of Medicine, Institute of Clinical Medicine, Finland
}

Both genetic and environmental factors play roles in the development of type 2 diabetes. The aim of the present study was to investi- gate the influence of the 19 known genetic risk loci and a positive family history $(\mathrm{FH})$ of diabetes on the effectiveness of lifestyle changes in the Finnish Diabetes Prevention Study (DPS). A total of 522 subjects with impaired glucose tolerance (IGT) were randomized into the control $(n=257)$ and intervention $(n=265)$ group. The median follow-up was 7 years. An oral glucose tolerance test was performed annually. The lifestyle intervention, aimed at weight reduction, healthy diet and increased physical activity, lasted for 4 years on average (range 1-6 years) and resulted in a significant reduction in diabetes incidence. A positive FH was reported by $66 \%$ and $61 \%$ of subjects in the intervention and control groups, respectively (NS). The persons with $\mathrm{FH}+$ and $\mathrm{FH}-$ did not differ in the mean genetic risk score (GRS) $(17.5+/-2.7$ vs. $17.6+/-2.8, \mathrm{p}=0.726)$. The 19 genetic risk variants, either alone or combined, did not have an effect on the incidence of diabetes. We also analyzed the effect of the 8 SNPs influencing insulin secretion (GRSsecr), but no significant contribution was observed, either. In the analysis including GRS (19 SNPs), FH and randomization group, adjusted for age, BMI, sex and center, only the randomization group had a significant effect on the incidence of diabetes, i.e. lifestyle intervention had a protective effect (HR 0.55, $95 \%$ CI $0.41 ; 0.75, \mathrm{p}<0.0001)$. Further analyses including also fasting and 2-h glucose and insulin values and their changes after 1 year intervention showed that conventional risk factors for diabetes remained the best predictors of diabetes. We conclude that lifestyle changes may overcome the impact of the known genetic factors and FH in the development of diabetes in persons with IGT.

\section{P044 \\ Benzylamine, a Protoalkaloid Found in Vegetables and Fruits, Increases the Expression of the Adipokine Apelin in 3T3-F442A Adipocytes}

Josep Mercader, Danielle Daviaud, Sandra Grès, Christian Carpéné

INSERM, U858, I2MR, IFR 150, University Toulouse I, France

Introduction: Benzylamine (Bza) is a protoalkaloid present in vegetables and fruits (e.g. $10 \mathrm{mg} / \mathrm{kg}$ in green salad). Although poor information is available on the biological actions of this dietary amine, it has been reported that Bza activates glucose transport when incubated in vitro at $0.1-1 \mathrm{mM}$ with rodent or human adipocytes. Bza is also able to improve glucose handling in diabetic rodents after chronic oral administration. It is a substrate of copper-containing enzymes, especially the semicarbazide-sensitive amine oxidase (SSAO), mainly expressed in adipocytes. In these cells, Bza mimics several insulin metabolic actions such as antilipolysis and lipogenesis.

Objective: To study whether Bza can regulate other adipocyte functions, such as adipokine expression.

Methods and Materials: Differentiated 3T3F442A cells were serum deprived and incubated for 6 hours with insulin, benzylamine and/or various pharmacological agents. Apelin and Resistin mRNAs were quantified by real-time PCR relative to $18 \mathrm{~S}$.

Results and Conclusions: As already reported, insulin strongly activated apelin expression and this was partially reproduced with 
Bza. Amine oxidation by SSAO appeared to be involved in such response, since the SSAO-inhibitor semicarbazide impaired Bza effect, but did not modify apelin expression in untreated or insulinstimulated cells. The effect of Bza was reproduced by tyramine, another dietary amine. However, 0.5 to $5 \mathrm{mM}$ tyramine was necessary to mimic the $0.1 \mathrm{mM}$ Bza-induced stimulation of apelin gene expression, again in a SSAO-dependent manner. No change in resistin expression was found in all the conditions above. High concentration $(1 \mathrm{mM})$ of hydrogen peroxide was inhibitory on apelin expression. In fact, this end-product of amine oxidation is generated at much lower doses upon Bza oxidation, while neither apelin nor insulin stimulated its production in $3 \mathrm{~T} 3 \mathrm{~F} 442 \mathrm{~A}$ cells. Thus, via SSAO activation, Bza partly mimics the up-regulating effect triggered by insulin on apelin expression.

\section{P045}

\section{Effect of Fatty Acids on ROS Production by Diabetic Neutrophils}

\section{Elaine Hatanaka1, Hosana Gomes Rodrigues ${ }^{2}$, Rui Curi ${ }^{2}$ \\ ${ }^{1}$ Cruzeiro Do Sul University-Säo Paulo, Brazil, ${ }^{2}$ University} of Sao Paulo, Brazil

Introduction: Elevated serum levels of FFA and ROS are involved in inflammation and insulin resistance in diabetes. The proposal of this study was to investigate the effect of $\mathrm{C} 18$ fatty acids on ROS production by diabetic neutrophils.

Methods and Material: The experimental type 1 diabetes was induced in rats by peritoneal injection of streptozotocin. Neutrophils were obtained by intraperitoneal lavage after the i.p. injection of sterile type II oyster glycogen solution.

Results: Using lucigenin-amplified chemiluminescence, a dosedependent increase in superoxide levels was observed in the treatment of control neutrophils with oleic, linoleic and g-linolenic acids (0-200uM). This effect was not observed for diabetic neutrophils: at low concentrations of fatty acids $(10-50 \mathrm{uM})$, diabetic neutrophils are not stimulated by fatty acids to release superoxide. However, at a high concentration of linoleic and g-linolenic acids $(100 \mathrm{uM})$, diabetic neutrophils are more responsive to release superoxide than control neutrophils. A curious effect is the kinetic profile of ROS production of the groups after treatment with fatty acids: while the control group reached its maximum ROS production capacity $5-10 \mathrm{~min}$, the peak production of the diabetic group occurred after $15-20 \mathrm{~min}$. In conclusion, we did not observe responsiveness of diabetic neutrophils to low concentrations of fatty acids, but we found increased and later production of superoxide anions of diabetic neutrophils in response to high concentrations of linoleic and linoleic acids.

Conclusion: The distinct responsiveness of diabetic neutrophils to fatty acids may be considered part of the scenario of diabetes physiopathology.

Financial Support: FAPESP, CNPq.

Key words: diabetes, fatty acids, neutrophils, ROS.

\section{P046 \\ Moderate Exercise Improves Leukocyte Function and Decreases Inflammation in Diabetes}

\author{
Elaine Hatanaka', Maicon Fabricio Belotto', \\ Juliana Magdalon², Hosana Gomes Rodrigues², \\ Marco Aurélio Ramirez Vinolo², Tania Cristina Pithon-Curi², \\ Rui Curi \\ ${ }^{1}$ Graduate Program in Human Movement Science-Cruzeiro \\ do Sul University-Säo Paulo, Brazil, ${ }^{2}$ Department of \\ Physiology and Biophysics, Institute of Biomedical \\ Sciences University-Säo Paulo, Brazil
}

Introduction: The genesis and progression of diabetes occur in part due to an uncontrolled inflammation profile with insulin resistance, increased serum levels of free fatty acids (FFA), pro-inflammatory cytokines and leukocyte dysfunctions.

Methods and Material: In this study, an investigation was made of the effect of a three-week moderate exercise regimen on a treadmill ( $60 \%$ of VO2 max, $30 \mathrm{~min} /$ day, 6 days a week) on inflammatory markers and leukocyte functions in diabetic rats.

Results: The exercise decreased serum levels of tumor necrosis factor-alpha (TNF) (6\%), neutrophilchemoattractant in inflammation 2 alpha/beta (CINC-2 /) (9\%), interleukin-1beta (IL-1) (34\%), interleukin-6 (IL-6) (86\%), C-reactive protein (CRP) (41\%) and FFA $(40 \%)$ in diabetic rats when compared with sedentary diabetic animals. Exercise also attenuated the increased responsiveness of leucocytes from diabetics when compared to controls, diminishing the release of ROS release by neutrophils (21\%) and macrophages $(28 \%)$. Exercise did not change neutrophil migration and the proportion of neutrophils and macrophages in necrosis (loss of plasma membrane integrity) and apoptosis (DNA fragmentation). Serum activities of creatinekinase (CK) and lactate dehydrogenase (LDH) were not modified in the conditions studied. Therefore, physical training did not alter the integrity of muscle cells.

Conclusion: Moderate physical exercise has marked antiinflammatory effects on diabetic rats. This may be an efficient strategy to protect diabetics against microorganism infection, insulin resistance and vascular complications.

Financial Support: FAPESP, CNPq.

Key words: diabetes, fatty acids, neutrophils, exercise. 


\section{P047 \\ Screening for Salmon and Cod Peptidic Fractions in Cell-Based Assays Reveal Biological Activities on Inflammation, Lipolysis and Glucose Uptake}

\author{
Noemi Boqué1,2, Laurie-Eve Rioux ${ }^{1,2}$, Charles Lavigne ${ }^{1,2}$, \\ Fida Hasan ${ }^{3}$, Roger McLeod ${ }^{4}$, Tom Gill ${ }^{3}$, André Marette ${ }^{1,2}$ \\ ${ }^{1}$ Institut Universitaire de Cardiologie et de Pneumologie \\ de Quebec, Canada, ${ }^{2}$ Institute of Nutraceuticals and \\ Functional Foods, Laval University-Quebec, Canada, \\ ${ }^{3}$ Department of Process Engineering and Applied \\ Sciencie, Dalhousie University, Halifax, Nova Scotia \\ Canada, ${ }^{4}$ Department of Biochemistry and Molecular \\ Biology, Dalhousie University, Halifax, NS, Canada
}

The consumption of fish proteins has been shown to improve insulin sensitivity in animal models of obesity and in obeses subjects [1-3]. One potential mechanism of action is through reducing chronic low grade inflammation, which is known to promote insulin resistance and diabetes in obese individuals. Recent in vivo studies from our lab have shown that consumption of fish protein hydrolysates (salmon, herring, bonito, mackerel) in a high fat diet reduce proinflammatory cytokine production in visceral adipose tissue of obese animals [4]. Therefore, the objective of the present study was to test in vitro the effects of salmon (SP) and cod peptides (CP) on different physiological functions including inflammation, lipolysis and glucose uptake in J774 macrophages, 3T3-L1 preadipocytes and L6 myocytes, respectively. iNOS - mediated NO production (a pro-inflammatory index) was significantly reduced (40-60\%) in LPS treated J774 macrophages incubated with SP. In 3T3-L1, we observed an increase in basal lipolysis in adipocytes incubated with CP and SP. Importantly both $\mathrm{CP}$ and SP fractions significantly increased basal and insulin stimulated glucose uptake in L6 muscle cells. In conclusion, these results show that CP and SP could enhance insulin sensitivity by decreasing NO production in macrophages as well as by increasing glucose uptake in myocytes. The lipolytic effect of CP and SP also suggest they could reduce lipid accretion in adipose tissue. Further studies will focus on the identification of the bioactive peptides with the aim of producing novel nutraceuticals or functional foods for prevention and management of type 2 diabetes and associated metabolic disorders. [1] Lavigne C et al., Am J Physiol Endocrinol Metabol 2000; 278: E491-E500. [2] Lavigne C et al., Am J Physiol Endocrinol Metabol 2001; 281: E62-E71. [3] Ouellet V et al., Diabetes Care 2007; 30: 2816-21. [4] Pilon G et al., Metab. Clin. Exp. in revision.

\section{P048 \\ Oral Tributyrin Improves Muscle Insulin Sensitivity, Production of Leptin by Adipose Tissue and Attenuates the Release of Inflammatory Cytokines by Macrophages}

Marco Aurelio Vinolo', Hosana Rodrigues' ${ }^{1}$ Fabio Sato', Thais Lima', Sandro Hirabara², Rui Curi ${ }^{1}$

${ }^{1}$ Institute of Biomedical Sciences, University of Säo Paulo, Brazil, ${ }^{2}$ Postgraduate Program in Human Movement Sciences, Institute of Phisycal Activity Sciences and Sports, Cruzeiro do Sul University, Brazil

Previous study has shown that oral butyrate prevents the development of insulin resistance and obesity in mice. The aim of this study was to evaluate the effects of tributyrin (a pro-drug of butyrate) on muscle metabolism and production of mediators by adipocytes (leptin and IL-6) and macrophages (CINC-2, IL-1, IL-6, TNF- $\alpha$ and nitric oxide). Adult male Wistar rats were treated by oral route with tributyrin (Tb) at 0.5 and $2.0 \mathrm{~g} / \mathrm{Kg}$ of b.w. during 2 weeks. Soleus muscles were then collected and assayed for glycogen synthesis, glucose uptake and oxidation. White adipocytes isolated from epididimal fat pads and peritoneal macrophages were incubated and evaluated for production of mediators. Body weight, food and water intake, fasting glycemia and hepatic enzyme activities (AST and ASP) were analyzed but no influence of $\mathrm{Tb}$ treatment was observed. An increase in soleus muscle sensitivity to insulin was observed in animals that received $\mathrm{Tb}$. The treatment with $\mathrm{Tb}$ also elevated the content of phosphorylated AKT in soleus muscle in response to insulin and prevented the insulin resistance induced by incubation in vitro with palmitate. Production of leptin by adipocytes was elevated. Attenuated production of cytokines (CINC-2, IL-1, IL-6 and TNF- $\alpha$ ) and nitric oxide was observed in macrophages stimulated or not with LPS. Results reported herein are indicative that $\mathrm{Tb}$ modulates the expression of mediators that are involved in the development of diabetes and the muscle response to insulin suggesting that this compound may be further studied as an alternative to prevention of type 2 diabetes and inflammatory disease.

\section{P049 \\ Oral Supplementation of Oleic and Linoleicacids Modulate Inflammatory Response in Diabetic Rats
Hosana Rodrigues ${ }^{1}$, Marco Aurelio Ramírez Vinolo', Camila Bento de Lima1', Sandra Farsky', Rui Curi ${ }^{1}$
${ }^{1}$ Department of Physiology and Biophysics/Sao Paulo, Brazil, ${ }^{2}$ Department of Clinical and Toxicological Analysis, Brazil

Objective: To investigate the effects of oral administration of oleic (OLA) and linoleic (LNA) acids on neutrophil function and inflammatory response in diabetic rats.

Methods: The diabetes was induced by intraperitoneal administration of streptozotocina ( $65 \mathrm{mg} / \mathrm{Kg}$ body weight). OLA and LNA were administered by gavage $(0.22 \mathrm{~g} / \mathrm{Kg}$ body weight) for 10 days. 
The migration of cells was analyzed in vivo with the air pouch assay, in which the number of cells and the concentration of cytokines (CINC-2 alpha/beta, IL-1 $\beta$ ) in exudates were quantified. The interaction between leukocytes and endothelium was assigned by intravital microscopy. The expression of L-selectin and $\beta 2$-integrin on neutrophils was analysed by flow citometry. ANOVA and Dunnett post test were used for statistical analysis $(\mathrm{p}<0.05)$.

Results: LNA supplementation increased the number of cells ( 5 fold), the concentrations of CINC-2 alpha/beta (21 fold) and IL-1beta ( 80 fold) in the air pouch. OLA presented a stimulatory effect on IL-1beta (20 fold increase). OLA augmented (16\%) the expression of $\beta 2$-integrin in the surface of leukocytes, the rolling (70\%) and the number of adhered cells (66\%) to the endothelium. LNA had no effect on these parameters.

Conclusion: LNA supplementation increased the inflammatory response of neutrophil (augmented the migration and the production of pro-inflammatory cytokines), but did not change the interaction between leukocytes and the endothelium. On the other hand, OLA supplementation elevated the interaction between cells and endothelium. These results show that these fatty acids can exert pro-inflammatory effects in different ways in the diabetic state.

Financial support: FAPESP, $\mathrm{CNPq}$ and CAPES.

\section{P050 \\ Inefficient Fat Oxidation in Peroxisomes Protects Against Obesity and Glucose Intolerance - A Mechanism for Fish Oil Effects on Glucose and Lipid Metabolism}

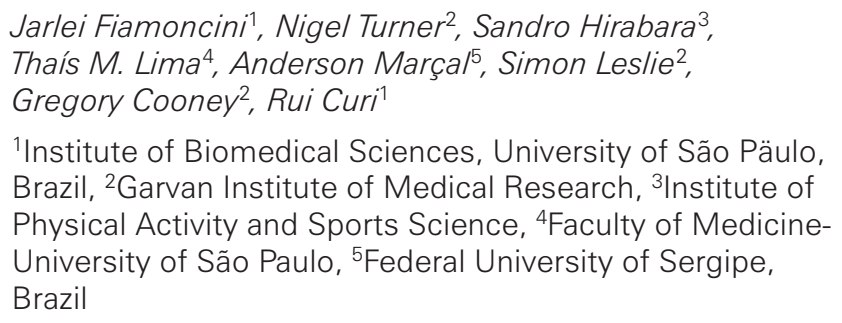

Herein we report a study on the modulation of glucose and lipid metabolism by different concentrations of fish oil and lard in the diet. Swiss mice were fed for 8 weeks on diets containing either fish oil at $4 \%$ (NFO) or $40 \%$ (HFO) and lard at $4 \%$ (NL) or $40 \%$ (HL). Glucose and lipid metabolism were studied. Fish oil fed animals weighed $\sim 10 \%$ less than corresponding lard-fed animals, and also displayed smaller epididymal and retroperitoneal fat pad $(\mathrm{p}<0.05)$. The lean phenotype observed in the HFO group was achieved even though this group had increased caloric intake when compared to the HL group and despite fish oil-fed mice showed lower oxygen consumption when compared with lard-fed groups $(\mathrm{p}<0.01)$. NFO and HFO exhibited a better response during a Glucose Tolerance Test than lard fed animals and $60 \%$ lower triacylglycerol levels in liver than lard-fed counterparts. Furthermore, despite lower complete oxidation of palmitate in liver homogenates from NFO and HFO animals, a 50\% higher level of acid soluble metabolites (ASM) were generated from palmitate in these groups, indicating high activity of beta-oxidation. Hepatocytes isolated from mice treated with Wy-14,643 - a strong PPAR agonist also showed lower complete oxidation of palmitate, but increased ASM generated from this fatty acid, when compared to hepatocytes isolated from Control mice. The activity of the key peroxisomal enzyme, acyl-CoA oxidase and other enzymes involved in beta-oxidation were also substantially enhanced in the liver of fish oil fed mice, as well as the content of PMP 70 - a peroxisome marker. Based on these results we can conclude that inefficient peroxisomal oxidation of fatty acids in liver is an important contributor to the lower fat accumulation and better glucose tolerance in n-3 PUFA fed mice.

P051

\section{The rs9939609 Polymorphism in the FTO Gene is Associated with Fat and Fiber Intakes in Women with Type 2 Diabetes}

\author{
Thais Steemburgo ${ }^{1}$, Mirela J. Azevedo ${ }^{1}$, Jorge L. Groos ${ }^{1}$, \\ Fermín Milagro ${ }^{2}$, Javier Campión ${ }^{2}$, J. Alfredo Martínez ${ }^{2}$ \\ ${ }^{1}$ Endocrine Division, Hospital de Clínicas De Porto Alegre, \\ Universidade Federal do Rio Grande do Sul, Brazil, \\ ${ }^{2}$ Institute of Nutrition and Food Sciences, Universidad of \\ Navarra-Pamplona, Spain
}

The A risk allele of the rs9939609 (A/T) polymorphism in the fat mass and obesity associated (FTO) gene has been associated with obesity, type 2 diabetes, and appetite regulation. Therefore, the aim of study was to evaluate possible associations of FTO rs9939609 with dietary factors in patients with type 2 diabetes. This cross-sectional study was conducted in 236 outpatients with type 2 diabetes (age 60.0 \pm 10.3 years; diabetes duration $12.7 \pm 8.2$ years; $53.4 \%$ females) who was genotyped for the FTO rs9939609 polymorphism (ABI PRISM 7000 Real-Time PCR System). Patients underwent clinical and laboratory evaluations and 3-day weighed-diet records. Data from dietary intake were categorized as HIGH or LOW based on median values. The AA genotype in the FTO gene was positively associated with HIGH fat $(>34 \%$ energy; OR $=2.17 ; 95 \%$ CI $1.02-4.63 ; \mathrm{P}=0.044)$ and LOW fiber intakes $(<16 \mathrm{~g} /$ day; $\mathrm{OR}=2.43 ; 95 \% \mathrm{CI} 1.05-5.58 ; \mathrm{P}=$ 0.037 ), adjusted for gender, BMI, total energy intake, and HbAlc. When gender was taken into account, these associations remained significant only for females regarding fat $(\mathrm{OR}=9.73 ; 95 \%$ CI 2.12 44.66; $\mathrm{P}=0.003)$ and fiber intakes $(\mathrm{OR}=4.28 ; 95 \% \mathrm{CI} 1.14-16.06 ; \mathrm{P}$ $=0.031)$ adjusted for the same variables. In conclusion, women with type 2 diabetes carriers of the AA genotype of the rs9939609 polymorphism in the FTO gene had increased fat and decreased fiber consumptions, independently of BMI. 
Cancer

\section{P052}

\section{Cocoa Flavonoids Induce CYP1A1 Overexpression in Breast Cancer Cells}

\author{
Carlota Oleaga1, Miriam Garcia', Anna Solé1, \\ Carlos J. Ciudad ${ }^{1}$, María Izquierdo-Pulido ${ }^{2}$, Véronique Noé1 \\ ${ }^{1}$ Departamento de Bioquímica y Biología Molecular \\ Facultad Farmacia Universidad de Barcelona, Spain, \\ 2Departamento de Nutrición y Bromatología. Facultad \\ Farmacia Universidad de Barcelona, Spain
}

Cocoa and their products are sources of flavonoids and contribute in a significant proportion to the amount of dietary antioxidants. There is substantial experimental evidence that connects dietary flavonoids with the prevention of chronic diseases, such cardiovascular diseases, diabetes or cancer. To further evaluate the role of cocoa flavonoids in cancer prevention, a functional genomic analysis was performed to study the effect of a polyphenolic cocoa extract (PCE) in the human breast cancer cell lines MCF-7 and SKBR3. Thus, changes in mRNA levels were determined using the specific Stress \& Toxicity PathwayFinder ${ }^{\text {TM }}$ RT2Profiler ${ }^{\mathrm{TM}}$ PCR Arrays. Upon incubation with PCE, 1 gene was underexpressed and 7 were overexpressed in MCF-7 cells whereas 9 genes were overexpressed in SKBR3 cells $(\mathrm{p}<0.05)$. Among the differentially expressed genes in both cell lines, CYP1A1 was chosen for further validation. The changes in expression for CYP1A1 upon incubation with PCE were confirmed at mRNA and protein levels. The roles of ER and HER2 in CYP1A1 induction were analyzed by means of the siRNA technology. We determined that ER triggered CYP1A1 induction through the AhR pathway in MCF-7 cells, whereas HER 2 modulated CYP1A1 over expression in response to cocoa extract in SKBR3 cells. To analyze a possible crosstalk between the different signalling pathways, AhR, ER; and HER2 proteins levels were determined by Western blot. The results showed that a crosstalk mechanism involving ER and AhR was activated upon incubation with PCE. Finally, the combination of PCE with tamoxifen was synergistic in both cell lines.

\section{P053}

\section{1,25-Dihydroxyvitamin D3 Inhibits the Expression of the Protumourigenic SPROUTY-2 Gene in Colon Cancer}

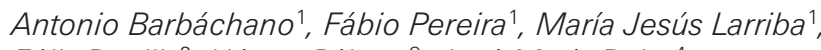
Félix Bonilla², Héctor Pálmer", José María Rojas", Alberto Muñoz 1

"Instituto de Investigaciones Biomédicas "Alberto Sols"Madrid, Spain, ${ }^{2}$ Hospital Universitario Puerta de Hierro, Majadahonda-Madrid, Spain, ${ }^{3}$ Vall d'Hebron Institut d’Oncología-Barcelona, Spain, ${ }^{4}$ Centro Nacional de Microbiología, Instituto de Salud Carlos III, MajadahondaMadrid, Spain

Introduction: Many epidemiological and preclinical studies suggest vitamin D compounds confer protection against colon cancer. We have reported that 1, 25-dihydroxyvitamin D3 (1,25 (OH) 2D3) inhibits the proliferation and promotes the differentiation of human colon cancer cells via the induction of the adhesion molecule E-cadherin and the antagonism of the Wnt/beta-catenin pathway. SPROUTY-2 (SPRY2) protein is an intracellular modulator of several growth factor tyrosine kinase receptors and therefore of cell growth and differentiation.

Materials and Methods: Human colon cancer SW480-ADH cells were cultured in DMEM. To knock-down gene expression, cells were infected with lentiviral particles containing a U6 promoter driving a shRNA targeting the respective RNA (Mission ${ }^{\circledR}$ shRNA lentiviral particles against human SPRY2, E-cadherin or scramble negative control, Sigma). To investigate SPRY2 expression in human colon tumours we considered sporadic cases if no clinical antecedents of Familial Adenomatous Polyposis were reported. Patients with clinical criteria of hereditary non-polyposis colorectal cancer (Amsterdam criteria) were excluded.

Results: SPRY2 expression in colon cancer cells was inhibited by $1,25(\mathrm{OH}) 2 \mathrm{D} 3$ through E-cadherin-dependent and -independent mechanisms. In turn, SPRY2 decreases both the basal and 1,25(OH)2D3-induced expression of E-cadherin, leading to the inhibition of the adhesive epithelial phenotype of colon cancer cells. Moreover, SPRY2 knock-down increases CDH1/E-cadherin expression and, reciprocally, CDH1/E-cadherin knock-down increases that of SPRY2. Consistently, SPRY2 and E cadherin protein levels inversely correlate in colon cancer cell lines. In colon cancer patients, SPRY2 is up-regulated in undifferentiated high grade tumours and at the invasive front of low grade carcinomas and correlates inversely with that of E-cadherin.

Conclusions: Our data show that 1,25(OH)2D3 represses SPRY2, a negative regulator of E-cadherin that promotes dedifferentiation and invasion of colon cancer cells. These results reflect an unanticipated tumourigenic activity and potential of SPRY2 as a marker of malignancy, and a novel protective effect of 1,25 (OH) 2D3 against this neoplasia. 


\section{P054}

Nutritional Genomics for the Determination of the Mechanism of Action of the Beneficial Effect of Bioactive Extracts in Tumor Progression

Susana Molina1, Margarita González-Vallinas', Mónica R. García-Risco², Tiziana Fornari², Susana Santoyo ${ }^{2}$ Guillermo Reglero ${ }^{1,2}$, Ana Ramírez de Molina ${ }^{1}$

${ }^{1}$ Institute IMDEA Food (Institute for advances studies of Madrid), Madrid, Spain, ${ }^{2}$ Food Science Department, Universidad Autónoma de Madrid, Madrid, Spain

Introduction: Much effort is dedicated to the development of herbal therapeutics in cancer. Extracts from common herbs possess bioactivities such as antioxidant or anti-inflammatory properties, and natural supplements with antioxidant activity have been reported to reduce the incidence or mortality of gastrointestinal tumors. However, different antioxidants display different effects and clinical trials are controversial, maybe due to the lack of knowledge of their specific mechanism of action.

Methods: Supercritical fluid extracted herbs with known antioxidant activity (HE) were used to analyze their effect on human colon cancer cells and determine its mechanism of action. Antiproliferative effect was analyzed by MTT assay in SW620 and HT-29 cells determining growth inhibition (GI50), citostacity (TGI50) and citotoxicity (LC50). Anchorage-independent growth assays were performed for cell transformation. Gene expression and ontology analysis after HE treatment were developed under different conditions according to the biological effects using Agilent G4112F Whole Human Genome Microarray and Ingenuity Pathways Analysis.

Results: HE exhibited dose-dependent viability suppression and inhibition of cell transformation. Gene expression analysis revealed that the most significant altered network was involved in cell death and protein degradation. Lipid and carbohydrate metabolism-related genes were also altered with a greater score than those related to oxidative stress or inflammatory response, suggesting a stronger effect of HE in tumors in which these networks are involved.

Conclusions: Phytochemical compounds display bioactivities pointing at them as promising supplements in nutritional-related diseases such as colon cancer. However, not all antioxidant phytochemicals are beneficial for all patients, and the analysis of the specific mechanism of action of each compound is necessary for including them in personalized treatments. Nutrigenomic analysis of phytochemical-induced modulation of gene expression is a powerful tool for understanding the biological effect of these products, and might result in an improvement of personalized therapies of cancer patients.

\section{P055 \\ Effects of Butyrate, Vitamin A and their Combination on Growth, Histone Acetylation and DNA Methylation Status, and Gene Expression of MCF-7 Human Breast Cancer Cells}

Thomas Prates Ong1, Marcia Kazumi Nagamine², Lucas Martins Chaible², María Lucia Zaidan Dagli², Aline De Conti', Camile Fontelles ${ }^{1}$, Alceu Afonso Jordão Jr. ${ }^{3}$, Helio Vannucchi ${ }^{3}$, Fernando Salvador Moreno ${ }^{1}$, Fabia De Oliveira Andrade ${ }^{1}$

${ }^{1}$ Faculty of Pharmaceutical Sciences of the University-Säo Paulo, Brazil, ${ }^{2}$ Faculty of Veterinary Medicine and Zootechny of the University of Säo Paulo, Brazil, ${ }^{3}$ Faculty of Medicine of Ribeirão Preto of the University of Säo Paulo, Brazil

Butyrate (BT) is a dietary short-chain fatty acid that presents histone deacetylase inhibitory actions. Retinoids regulate cell growth and differentiation. Combination of histone deacetylase inhibitors with retinoids has been proposed as a potential strategy for breast cancer control. The aim of this study was to investigate effects of BT $(1 \mathrm{mM})$, vitamin A (VA; 10microM) and their combination (BT+VA; $1 \mathrm{mM}+10 \mathrm{microM})$ in MCF-7 human breast cancer cells. Evaluated parameters included: cell growth (crystal violet assay); global histone 3 lysine 9 (H3K9) and histone 4 lysine 16 (H4K16) acetylation status (western blot); global DNA methylation status (dot-bot); retinoic acid receptor beta (RARbeta) and cellular retinol-binding protein1 (CRBP1) gene expression (qPCR); RARbeta gene promoter methylation status (MS-PCR) and cellular concentration of retinoids (HPLC). Compared to controls, represented by untreated MCF-7 cells, 34\%, $10 \%$ and $46 \%(p<0.05)$ growth inhibitions were observed after 120 hours of treatment with BT, VA and BT+VA, respectively. Compared to controls, MCF-7 cells treated with $\mathrm{BT}$ and $\mathrm{BT}+\mathrm{VA}$ presented a 10 -fold and 8-fold increase ( $<<0.05$ ), respectively, in H3K9 acetylation level after 96 hours of treatment, and a 2-fold increase $(p<0.05)$ in RARbeta expression after 120 hours of treatment. Compared to controls, BT and VA, alone or combined, did not alter $(\mathrm{p}>0.05)$ H4K16 acetylation level, global DNA methylation and CRBP-I gene expression after 96 and 120 hours of treatment. Untreated MCF-7 cells and those treated with BT and VA, alone or combined, presented RARbeta gene promoter predominantly methylated and undetectable levels of retinyl palmitate. The present data indicate that combination of BT with VA resulted in additive inhibitory effect on the growth of MCF-7 breast cancer cells. Inhibition of histone deacetylase activity and induction of RARbeta gene expression could represent important features of BT but not of VA anticancer effects.

Financial support: FAPESP (2008/58697-9; 2008/51742-9). 


\section{P056 \\ The $\omega-3$ Fatty Acid DHA Down Regulates the Expression of Genes Associated with Breast and Colorectal Cancers - A Whole - Genome Microarray Analysis}

Tonia Buonomo', Marika Massaro2, Egeria Scodittti ${ }^{2}$
María Annunziata Carluccio ${ }^{2}$, Raffaele De Caterina ${ }^{3}$,
Rosanna Martinelli ${ }^{1,4}$

${ }^{1}$ CEINGE Biotecnologie Avanzate, Naples, Italy, ${ }^{2}$ C.N.R.

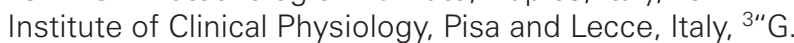
d'Annunzio" University and Center of Excellence on AgingChieti, Italy, ${ }^{4}$ Department of Biochemistry and Medical

Biotechnologies, Federico II University-Naples, Italy

Background: Inflammation is a critical component of tumor progression and many cancers arise from sites of infection, chronic injury, and inflammation, where immune cells secrete large amounts of pro-inflammatory cytokines able to recruit a wide range of immune cells. The aim of this study was to gain insights into the molecular basis of potential chemo preventive and therapeutic activities of the $\omega-3$ fatty acid DHA.

Methods and Materials: Human umbilical vein endothelial cells (HUVEC) were treated with $50 \mu \mathrm{mol} / \mathrm{L} \mathrm{DHA}$ for 48 hours and then stimulated with $5 \mathrm{ng} / \mathrm{mL}$ IL- $1 \beta$ for 3 hours. Total RNA from treated cells and controls were extracted using the RNeasy mini kit from Qiagen. RNA samples were analyzed quantitatively and qualitatively with a NanoDrop Spectrophotometer and an Agilent Bioanalyzer. RNA was labeled using Agilent low RNA Input Fluorescent Linear Amplification kit and purified by Qiagen's RNeasy mini spin column. Gene expression profile was then performed using an Agilent Whole Human Genome Oligo Microarray, covering 41K unique genes and transcripts. Slides were scanned and image data processed using the Agilent Feature Extraction software. The raw data were processed by GeneSpring ${ }^{\circledR} 10$ and differentially expressed RNA identified with the Benjamini and Hochberg False Discovery Rate $(\mathrm{p}<0.05)$ test. Functional and network analyses were performed by Ingenuity Pathways version 8.0 Analysis.

Results and Conclusion: The gene expression profile of HUVEC pre-treated with DHA and stimulated with IL-1 shows that fish oil regulates several cellular pathways. Among these DHA down regulates the expression of 18 genes associated to cancer. In particular DHA affects the expression of HSPB8 and SIK1, known to be associated with breast cancer and the down regulation of PDE5A and DNAJA1 involved in colorectal cancer. The outcome of this study is of great interest because of DHA implication in human breast and colorectal cancer prevention.

\section{Personalized Nutrition}

\section{P057 \\ Introducing Nutritional Genomics Teaching in Undergraduate Allied Health and Nursing Curricula}

Chandan Prasad

Texas Woman's University-Denton, United States

Although we have known for a long time the individual variations in response to nutrients, it was the completion of the Human Genome Project that exponentially increased our knowledge of reciprocal relationship between genes and nutrients and led to birth of a new discipline - Nutritional Genomics. Many of us use nutritional genomics to include nutrigenomics (how nutrients affect gene expression?) and nutrigenetics (how genetic variations affect nutrient response). A fairly high proportion of undergraduates with degrees in nursing and health sciences obtain clinical credentials (Registered Nurse, Registered Dietitian, and Certified Health Education Specialist) and work as healthcare providers. In this capacity they will have to have clear understanding of nutritional genomics and associated issues. The purpose of this presentation is not to explore future research opportunities in this area, but how to systematically incorporate teaching of basic understanding of nutritional genomics and associated legal, social and ethical issues in undergraduate curricula of health sciences. While there is no doubt that addition of a stand-alone course in nutritional genomics will be the ideal solution, most undergraduate curricula are packed with core or other mandated graduation requirement adding a new course is always a challenge. In the rest of my presentation, I am going to discuss the minimal learning objective sufficient to prepare students for educating others. Some of the learning areas that may help meet objectives may include: a) how information flows between nucleic acid and protein? b) How dietary chemicals alter gene expression and increase disease susceptibility? c) How nutrients may affect gene expression in utero? What are legal, social, and ethical issues associated with genetic testing and advice. Next, using examples from two diverse undergraduate curricula, one science intensive and other less science intensive- Dietetics and Family $\&$ Consumer Science- we will discuss how core-learning objectives should be incorporated. 


\section{P058}

\section{Bitter Taste Genetics and Food Preference in Italian Population}

Roberto Barale1, Maura Carrai', Daniele Campa², Fabio Caradonna4, Irene Catanzaro ${ }^{4}$, Federico Canzian², Salvatore Saccone ${ }^{3}$, Concetta Federico ${ }^{3}$, Salvatore Motta ${ }^{3}$

${ }^{1}$ Dipartimento di Biologia, Università di Pisa, Italy, ${ }^{2}$ German Cancer Research Center- Heidelberg, Germany,

${ }^{3}$ Dipartimento di Biologia Animale, Università di Catania, Spain, ${ }^{4}$ Dipartimento di Biologia Cellulare e dello SviluppoPalermo, Italy

Objective: To investigate the possible role of the polymorphic bitter taste gene, TAS2R38, known to be involved in the perception of the bitter synthetic chemical phenylthiocarbamide (PTC), in influencing food preference and body mass index (BMI).

Methods: Up to now more than 1500 university students (17-25 years old) at Catania, Cosenza, Rome, Palermo, Pisa, Parma, Chieti, and Trento University have been enrolled in the study. DNA was extracted from saliva, and genotyped by TaqMan assay for the most frequent polymorphism (PAV/AVI) of TAS2R38 gene. A possible association between genotype and food preference was assessed by administering a detailed questionnaire for food preferences and life style.

Results: 1) the entire population was found to be in HardyWeimberg equilibrium. 2) No significant difference in allele frequencies between Centre and South Italy was observed, neither between the isolated population of Garfagnana and the rest of Italy. 3) A significant difference was observed considering rare aplotypes. 4) A good correlation ( $80 \%$ ) between TAS2R38 polymorphism and capability to assess PTC bitterness was observed. 5) A suggestive, but none statistically significant correlation between BMI and genotype, particularly in male, $(\mathrm{p}=0.08)$ was obtained. 6) An indication that TAS2R38 polymorphism (genotype) is associated with the preference of some food such as: bitter chocolate $(\mathrm{p}=0.02)$, sprouts $(0.09)$, onion (0.03), whipped cream $(\mathrm{p}=0.09)$ was obtained. 7) In addition, the indication that bitter PTC assessment capability (phenotype) can be associated with the preference ordisliking of cabbage $(\mathrm{p}=0.015)$; bitter chocolate $(\mathrm{p}=0.10)$ sausages $(\mathrm{p}=0.05)$; garlic $(\mathrm{p}=0.08)$; onion $(\mathrm{p}=0.07)$; citrus fruits $(0.08)$, strong cheese $(\mathrm{p}=0.06)$ was also obtained.

Conclusions: We are aware that TAS2R38 is just one of the 25 (or more) human polymorphic gene involved in the perception of bitter taste or astringency of foods and that it appears to be particularly associated to PTC bitterness.

\section{P059 \\ Effect of Glutamine-Enriched Diet on Protein and Amino Acid Metabolism in Skeletal Muscle of Rat}

Milan Holecek, Miroslav Kovarik, Ludek Sispera, Pavel Siman

Charles University Prague, Medical Faculty in Hradec Kralove, Czech Republic

Rationale: Glutamine supplementation is frequently recommended in severe illness and in athletes. However, the information about safety and consequences of a long-term intake of glutamineenriched diet is limited.

Methods: Male Wistar rats $(n=40)$ were fed with a standard laboratory diet or glutamine-enriched diet. After three months, the effect of glutamine was examined in fed and overnight fasted animals. Protein synthesis in $\mathrm{m}$. soleus (slow-twitch muscle) and $\mathrm{m}$. tibialis (fast-twitch muscle) was examined by the "flooding method" using phenylalanine, proteolysis on the basis of chymotrypsine-like activity (CHTLA) of proteasome and cathepsine activities. Amino acid concentrations were estimated using HPLC, glutamine synthetase activity by a colorimetric assay. ANOVA and Bonferroni test were used for statistical analysis.

Results: There was no effect of a type of a diet on food intake and weights of the body and muscles. In the blood of animals fed by glutamine-enriched diet sacrificed in fed state we found higher concentrations of urea and glutamine, lower concentrations of glucose and triglycerides, and significant alterations in a number of amino acids. In both types of muscle we found higher concentrations of glutamine. The intake of glutamine-enriched diet had no effect on protein synthesis, CHTLA activity, and glutamine synthetase activity in muscles. However, a decrease in cathepsine-like activity was found in m. soleus. Comparison of fed and fasted animals revealed that the most of metabolic alterations induced by glutamine-enriched diet rapidly disappears. The exceptions were the prolonged decrease in glycine in plasma, and the decrease in glycine and increase in lysine in muscle.

Conclusions: Long term intake of glutamine-enriched diet has significant metabolic effects on the body.

Supported by Research Project MSM 0021620820.

\section{P060 \\ Association Between a Common Polymorphism (rs6564851) Near the BCMO1 Gene and Circulating Carotenoid Levels in Japanese Volunteers \\ Yoshihiro Shidoji, Masanori Urata, Roseline Yap Wai Kuan, Motofumi Masaki \\ University of Nagasaki, Japan}

More than a quarter-century ago, Dr. Cutler found a significant positive correlation between maximal life-span potential of different species and the concentration of carotenoids in serum (P.N.A.S., $81: 7627,1984)$. Unlike retinol in serum, circulating carotenoid levels 
in humans are well known to vary substantially among individuals, nations where the subjects live and seasons when blood collection is performed, suggesting that circulating carotenoid levels may depend on the amount of carotenoid intake. Recently, a genome-wide association study revealed that a common variation (rs6564851) near the $\beta$-carotene 15,15'monooxygenase (BCMO1) affects circulating levels of carotenoid in 3 Caucasian populations (Ferrucci et al.: Am. J. Human Genet., 84: 123, 2009), giving an idea that some sort of genetic backgrounds may influence carotenoid level in blood. Therefore, gene-environment interaction studies will provide new information in order to understand physiological regulation of circulating carotenoid level. In the present study, we evaluated the effects of rs6564851 polymorphism and dietary intake of carotenoids on plasma carotenoid levels in 92 Japanese adult volunteers (63 men and 29 women). PCR-RFLP analysis for rs6564851 clearly discriminated 3 genotypes (GG, GT, TT). Dietary intake information was obtained using semi-quantitative food frequency questionnaire (FFQ). Plasma carotenoids were measured separately by HPLC. The allele frequency (the $\mathrm{G}$ allele 0.824 and the $\mathrm{T}$ allele 0.175 ) of rs6564851 was consistent with the numbers $(0.833$ and 0.167$)$ for the Japanese population in the NCBI SNP database, in which these numbers are 0.467 and 0.533 for Caucasian and 0.358 and 0.642 for African, respectively. The average concentration of plasma $\beta$-carotene in the T-allele carriers was less than half of that in the GG homozygotes, whereas the amounts of carotenoid intake was not significantly different between these 2 genetic groups. In conclusion, we found a strong association between rs6564851 and circulating $\beta$-carotene level in Japanese population.

\section{P061 \\ MTOR Activity is Downregulated in Skeletal Muscle of Offspring of Rats Fed a High Fat Diet During Gestation and Lactation}

\author{
Lucas Carminatti Pantaleão, Tatyana Dias De Paula, \\ Emidio Marques De Matos-Neto, Gabriela Fullin, \\ Teodoro Rezende, Julio Tirapegui \\ University of São Paulo, Brazil
}

Introduction and Objectives: When fed a typical western diet during gestation and lactation, the increased peripheral insulin resistance seen in the offspring of rats can be linked to a marked reduction in skeletal muscle development. Under normal conditions, the signal transduction of the traditional insulin pathway results in an activation of the mammalian target of rapamycin (mTOR), a protein that acts as a positive effector of mRNA translation rate and myoblast differentiation. The present study aimed to evaluate the effect of maternal consumption of a high fat diet during gestation and lactation on the activity of the mTOR pathway in skeletal muscles in rat offspring.

Material and Methods: Sixteen pregnant rats were distributed into two groups with distinct diets: control $(n=8)$ and high-fat $(n=8)$. In the end of the lactation period, offspring samples were killed for excision and weighing of soleus and gastrocnemius muscles. A blood sample was also collected. Serum glucose, Insulin and IGF-1 concentrations were analyzed by spectrophotometry and xMAP. Western blotting was performed to analyze expression and phosphorylation of the proteins under study (4E-BP1, S6K-1 and mTOR).
Results and Conclusion: Relative muscle mass and mTOR phosphorylation were markedly reduced in the offspring of rats fed a high fat diet. However, the phosphorylation of downstream proteins in the mTOR pathway was not altered. The effect on mTOR activity can be explained by reductions in insulin sensitivity related to increased concentrations of insulin and glucose.

Financing: FAPESP, CAPES.

\section{P062}

\section{Hypotriglyceridemic Effect of Plantago Ovata Husk Optimized by Genetic Variants of FABP-2 and PAI-1}

\author{
Rosa María Valls Zamora', Anna Anguera Vila², \\ Rosa Solé Alberich ${ }^{1}$ \\ ${ }^{1}$ Unitat de Recerca en Lípids i Arteriosclerosi, CIB-Reus, \\ Spain, ${ }^{2}$ Rottapharm, Madaus, SL, Barcelona, Spain
}

Introduction: Fiber intake is associated with a reduced risk of cardiovascular disease (CVD). In particular, a type of soluble fiber, Plantago ovata husk (Po-husk) reduces levels of triglycerides (TG) in subjects infarcted and obese Zucker rats. Therefore, the objective is to evaluate the effect of Po-husk on plasma TG levels in hypercholesterolemic subjects on the basis of genes involved in CVD.

Methods: Multicenter, randomized, double-blind, parallel in Spain, France and Holland, with subjects between 43 and 67 years with LDL-c $\leq 4.88 \mathrm{mmol} / \mathrm{L}$ and $\mathrm{TG}<3.97 \mathrm{mmol} / \mathrm{L}$, who consumed 14 $\mathrm{g} / \mathrm{d}$ Po-husk $(\mathrm{n}=126)$ or microcrystalline cellulose (placebo, $\mathrm{n}=128$ ) with a low saturated-fat diet during 8 weeks. Lipid profile, biomarkers and some polymorphisms (SNPs) of genes related to CVD risk were determined.

Results: Compared with baseline, the Po-husk group reduced $16.6 \%$ plasma TG $(\mathrm{P}=0.01)$ and $-21.6 \%$ compared with the placebo group $(\mathrm{P}=0.01)$. Significant associations were observed between treatment and the SNPs of the genes of plasminogen activator inhibitor type 1 (PAI-1, rs1799768) and Fatty Acid Binding Protein type 2 (FABP-2, rs1799883). After 8 weeks of Po-husk consumption, the homozygotes $\mathrm{G}$ of PAI-1 show reductions (mean $\pm \mathrm{SD}$ ) $-0.44 \pm .20$ $\mathrm{mmol} / \mathrm{L}(28.1 \%)$ versus (vs) $-0.31 \pm 0.12 \mathrm{mmol} / \mathrm{L}(23.1 \%)$ in DEL $(\mathrm{P}=$ $0.01)$. In FABP-2, the homozygous A show a decrease of $-0.85 \pm 0.19$ $\mathrm{mmol} / \mathrm{L}(46.2 \%)$ vs $-0.21 \pm 0.10 \mathrm{mmol} / \mathrm{L}(20.4 \%)$ in $\mathrm{G}(\mathrm{P}=0.03)$. The hypotriglyceridemic mechanism proposed is the reduction of production and accumulation of TG in addition to the inhibition of fatty acid synthase in the liver, causing a delay in the intestinal absorption of TG.

Conclusion: We verified the unique hypotriglyceridemic effect of Po-husk. If the frequency of the variants of these genes, 0.5, is confirmed in the general population, Po-husk would optimize the hypotriglyceridemic response. 
Inflammation

\section{P063}

A Paracrine Loop Between Adipocytes and Macrophages in Adipose Tissue: An In Vitro Model to Study Obesity-Induced Inflammation

Victor Pallarès, Anna Castell-Auví, Lídia Cedó,

Montserrat Pinent, Anna Ardèvol, Mayte Blay

Universitat Rovira i Virgili-Tarragona, Spain

Introduction: Obesity is associated with a state of chronic, lowgrade inflammation that targets the adipose tissue. The adipose tissue is an endocrine organ that secretes many proinflammatory factors and includes various cell types such as lipid laden adipocytes and macrophages amongst others. TNF and MCP-1 have been shown up regulated in obese adipose tissue and adipocyte-FFA and macrophage-TNF establish a vicious circle that initiates and aggravates inflammation. In vitro histotypic culture models of adipose tissue have been yet used to study adipose tissue biology and cell molecular mechanisms with good results.

Methods: In this study, we used an in vitro coculture model to asses inflammation in obesity. We cocultured murine RAW 264.7 monocytes with fully differentiated 3T3-L1 adipocytes in an insert culture system. Levels of bioactive cytokines MCP-1 and IL-6 by enzyme immunoassay and FFA by colorimetric assay were measured. And also, the transcriptomic profile of 91 genes was analyzed by TLDA ${ }^{\circledR}$ (TaqMan Low density Array). Relative expression levels of genes involved in inflammation pathways such as interleukins, cytokines, chemokines, transcription factors and MAPKs, oxidative stress related genes, genes related to apoptosis, adhesion molecules, matrix degradation and metabolism were assessed.

Results: Coculture system allowed the evaluation of which proinflammatory mediators are up regulated by the coculture and compare them with the control isolated cells. Gene expression profile was described for each cell line alone and in coculture.

Conclusion: We provide evidence that the coculture system of adipocytes and monocytes mimetize some of the features of adipose tissue biology. Although preliminary results, this model might be used as a tool for screening the effectiveness of putative anti-inflammatory compounds obesity- specific.

\section{P064 \\ Carotenoids Differentially Modulate Nitric Oxide Availability and Reduce TNF - Induced Monocyte-Endothelial Interaction}

\author{
Pamela Di Tomo ${ }^{1,3}$, Raffaella Canali², \\ Doménico Ciavardelli1,3, Sara Di Silvestre 1,3, Elena Azzini², \\ Annalisa Giardinelli1,3, Caterina Pipino ${ }^{1,3}$, \\ Natalia Di Pietro ${ }^{1,3}$, Fabio Virgili2 ${ }^{2}$, Assunta Pandolfi, ${ }^{1,3}$ \\ ${ }^{1}$ Department of Biomedical Sciences-Chieti, Italy, \\ ${ }^{2}$ National Research Institute for Food and Nutrition \\ (INRAN)-Roma, Italy, ${ }^{3}$ Aging Research Center, Ce.S.I. \\ "Gabriele d'Annunzio" University Foundation, Chieti- \\ Pescara, Italy
}

Introduction: The activation of endothelium and subsequent enhanced monocytes adhesion and inflammatory response in blood vessels has been linked to cardiovascular disease. Nitric Oxide (NO) plays vascular anti-inflammatory action by inhibiting vascular adhesion molecules expression. Epidemiological studies suggest that carotenoid rich diets are associated with suppression and/or delay of cardiovascular disease progression, by a mechanism still partially known. We hypothesized that dietary carotenoids decrease vascular inflammatory response by increasing endothelial Nitric Oxide (NO) bioavailability.

Methods and Materials: Human Umbilical Vein Endothelial Cells (HUVEC) was incubated with carotenoids (carotene [BC], lycopene [Lyc] and cryptoxanthin [Cry], 0.5-2.5 $\mu \mathrm{mol} / \mathrm{L}$ ) for 24 hours. HUVEC were then stimulated with Tumor Necrosis Factoralpha (TNF $1 \mathrm{ng} / \mathrm{mL}$ ) for 16 hours with subsequent determination of vascular cell adhesion molecules (VCAM-1), intercellular cell adhesion molecules (ICAM-1), E-Selectin protein levels. The functional consequences of HUVEC treatment with carotenoids on human monocytoid cell (U937 line) were also evaluated by adhesion assay. NF-kB pathway involvement and NO release were also evaluated.

Results: BC and Lyc (1-2.5 mol/L) down regulated TNF induced NF B-dependent adhesion molecule expression and monocyteHUVEC interaction. In parallel, BC and Lyc $(2.5 \mathrm{~mol} / \mathrm{L})$ time-dependently increased NO bioavailability. TNF increased monocyte-HUVEC interaction, but not total adhesion molecules expression, NF-kB pathway activation and NO release, were inhibited by Cry.

Conclusions: Our observations provide the evidence that all carotenoids studied inhibit

TNF increased monocytes adhesion to HUVEC. BC and Lyc exerted their anti-inflammatory action possibly by increasing NO bioavailability and this may contribute to explain why carotenoidrich diets are associated with reduced risk of cardiovascular disease. 
P065

Activity of C-reactive Protein, IL-6 and TNFalfa in Obese Young People and Its Association with Lipid Profile

\author{
Adelaida Sara Minia Zepeda Morales ${ }^{1}$, \\ Trinidad García Iglesias ${ }^{1}$, Teresa Arcelia García Cobián², \\ Pedro Ernesto Sánchez Hernández1, Omar Eduardo \\ Fernández Vargas², Sylvia Elena Totsuka Sutto², \\ Leonel García Benavides², Ernesto Germán Cardona² \\ ${ }^{1}$ Laboratorio de Inmunología-Guadalajara, México, \\ ${ }^{2}$ Unidad de Investigación Cardiovascular, México
}

Obesity (OB) is an abnormal and excessive accumulation of fat, which can be harmful to health. OB has been defined as an inflammatory state based on amount of body fat. Adipose tissue functions are: reserve energy, production of hormones and proinflammatory cytokines such as IL-6 and TNF- $\alpha$, which stimulate the production of $\mathrm{C}$-reactive protein (CRP). It could increase the risk of cardiovascular diseases. The aim of this study was to evaluate the concentration of CRP, IL-6, TNF- $\alpha$ and its association with cholesterol, triglycerides and HDL in obese young people. We assessed 47 individuals at 18 to 25 years old divided into two groups: OB and controls $(\mathrm{C})$ body mass index (BMI) was evaluated and peripheral blood was obtained, according to Helsinki`s declaration. Triglycerides and HDL were measured by enzymatic colorimetric methods. CRP was measured by immunoassay, IL- 6 and TNF- $\alpha$ by ELISA. The OB and C groups did not show significant differences between ages (20.55 \pm 2.42 vs $20.61 \pm 1.97$ ); BMI was $36.58 \pm 4.91$ vs $22.27 \pm 2.41$. We found statistically significant difference in triglycerides concentration (119.16 \pm 36.37 vs $97.46 \pm 34.14)$, HDL $(48.77 \pm 21.51$ vs $92.04 \pm 57.69)$ and IL-6 $(1.13 \pm 0.73$ vs $0.722 \pm 1.07)$. We did not found difference in CRP $(5.82 \pm 1.53$ vs $5.27 \pm 0.66)$. We found a low increase in TNF- $\alpha$ concentration in OB, IL-6 showed a direct correlation with BMI, CRP and triglycerides in obese group compared with controls. Obesity is intimately related to triglycerides and low levels of HDL in the OB group. A strong correlation was observed between IL- 6 and CRP, which suggest that obesity acts as an inflammatory state and it increases the risk of an irregular immune response.

\section{P066}

\section{An Inflammatory Role of Twist 1 in Human White Adipocytes}

\author{
Amanda Pettersson ${ }^{1}$, Niklas Mejhert', Erik Näslund², \\ Anne Bouloumie 3 , Ingrid Dahlman', Jurga Laurencikiene1, \\ Peter Arner ${ }^{1}$, Mikael Rydén ${ }^{1}$ \\ ${ }^{1}$ Karolinska Institutet, Dep. of Medicine-Stockholm, \\ Sweden, ${ }^{2}$ Karolinska Institutet, Dep. of Clinical Science, \\ ${ }^{3}$ Institut National de la Santé et de la Recherche Médicale \\ (INSERM), U858-Toulouse, France
}

Objective: Twist1 is a transcription factor which is highly expressed in murine brown and white adipose tissue and negatively regulates fatty acid oxidation in brown adipocytes of mice. The role of twistl in white adipose tissue is not known.
Research design and Methods: The expression pattern of twist1 in human white adipose tissue, its regional distribution and cellular source was determined by qRT-PCR. The effect of twist1 siRNA on fatty acid oxidation, lipolysis, adipokine secretion and mRNA expression was determined in human adipocytes. Binding of twist 1 to specific promoters in human adipocytes was confirmed by chromatin immune-precipitation (ChIP).

Results: Twist1 was highly expressed in human adipose tissue compared to a set of other tissues and predominantly found in white adipocytes. Twist1 levels increased during in vitro differentiation of human preadipocytes. Gene silencing of twist1 in human white adipocytes had no effect on lipolysis or glucose transport. Unexpectedly, and in contrast to results in mice, twist1 RNAi reduced fatty acid oxidation. Furthermore, the expression and secretion of the inflammatory factors interleukin- 6 and monocyte chemoattractant protein-1 were down-regulated by twist1-silencing. This was achieved via direct transcriptional regulation since twist1 binding was confirmed by ChIP analysis.

Conclusions: This is the first study that delineates the role of twist 1 in white adipocytes. Twist1 may play a role in adipose inflammation since it regulates the secretion of inflammatory adipokines via direct transcriptional effects. In addition, twist1 may, in contrast to brown fat cells, be a positive regulator of fatty acid oxidation in human white adipocytes.

\section{P067 \\ Diet-induced Obesity Inhibits NF-kappab Activation in LPS-Stimulated Peritoneal Macrophages of Wistar Rats}

\author{
María Carolina Borges¹, Marco Vinolo², Mónica Yamada1, \\ Patricia Jacob', Tatiane Fujiii', Julio Tirapegui ${ }^{3}$, \\ Ricardo Fock', Rui Curi², Primavera Borelli4, \\ Marcelo Rogero 1 \\ ${ }^{1}$ School of Public Health, São Paulo University, Brazil, \\ ${ }^{2}$ Department of Physiology and Biophysics, \\ Institute of Biomedical Sciences-Säo Paulo, brazil, \\ ${ }^{3}$ Department of Food Science and Experimental \\ Nutrition, Faculty of Pharmaceutical Sciences-Säo Paulo, \\ Brazil, ${ }^{4}$ Department of Clinical and Toxicological \\ Analyses, Faculty of Pharmaceutical Sciences-Säo Paulo, \\ Brazil
}

Despite being a proinflammatory condition, obesity is associated with an increased susceptibility to bacterial infection. The aim of this study was to investigate the effect of diet-induced obesity (DIO) on nuclear factor-kappaB (NFkB) activation and on Wistar rats were divided in two groups: control group $(\mathrm{n}=6)$, fed ad libitum with an American Institute of Nutrition diet (AIN - 93M), and DIO group ( $\mathrm{n}=$ 6), fed with AIN-93M diet enriched with lard (30\% w/w). After 12 weeks, rats were euthanized. Peritoneal macrophages were collected, incubated with lipopolysaccharides (LPS) $(1 \mu \mathrm{g} / \mathrm{mL})$ and it was evaluated: (1) NFKB activation by electrophoretic mobility shift assay and (2) protein expression/phosphorylation of IB kinase (IKK) and inhibitor B IB by Western blotting, 30 minutes after LPS incubation; (3) the levels of interleukin (IL)-1, IL-6 and tumor necrosis factor (TNF) in the culture supernatant by an enzyme-linked immunosor- 
bent assay, 24 hours after LPS incubation. Results were previously analyzed for normality and differences between groups was determined by a t-test for independent variables, with $=0.05$. Body mass, weight gain and periepididimal fat depot increased significantly in DIO group when compared to control group $(\mathrm{p}<0.05)$. Stimulation with LPS did not increase NFB activation and phospho: total IB ratio in DIO group when compared to control group $(\mathrm{p}<0.05)$. The ratio between phospho and total IKK was significantly lower in LPSstimulated peritoneal macrophages from DIO group in comparison with control group $(p<0.05)$. DIO resulted in suppression of IL-1 synthesis, but did not impact on IL-6 and TNF production by peritoneal macrophages. The present study demonstrated that peritoneal macrophages from rats with DIO have a blunted inflammatory response when stimulated with LPS, which may be partially linked to an inhibition of NF- $\mathrm{KB}$ activation.

\section{P068 \\ Development of an Integrated Method in Vitro to Evaluate Bioavailability and Immunomodulator Properties of Food and Food Ingredients}

\begin{abstract}
Lidia Tomás Cobos, Blanca Viadel, Ma Teresa Navarro, Alejandro Rodrigo, José Enrique Carreres, David Tomás
\end{abstract}

Ainia Centro Tecnológico-Valencia, Spain

Food intake and nutrient exposure are key environmental factors in the pathogenesis and progression of diet-related diseases. Recently it is growing the interest on natural foods as a source of bioactive compounds. Inflammation is a common component of many diseases such as cardiovascular diseases, Crohn's disease, diabetes type I, metabolic syndrome or obesity. Identification of immunomodulator nutrients is becoming an important issue. To highlight the role of bioactive substances in the prevention of diseases, it is crucial to understand the factors affecting its release from food matrix, what amounts are absorbed and their interaction with the target tissues. The aim of the present study is to develop an in vitro system, consisting of simulated gastrointestinal digestion and Raw 264.7 and THP1 cells, to evaluate the immunomodulator properties on food and food ingredients. The diagnostic system has been designed taking on account the bioavailability of food components and the interaction gene-compounds. The food samples are treated by an in vitro digestion assay. The simulated gastro-intestinal digestion includes a process of dialysis with an enzyme treatment in two stages, first with pepsin at $\mathrm{pH}$ $=1.8-2.5$ (gastric digestion) and second with pancreatin-bile extract at neutral $\mathrm{pH}$ (intestinal digestion). Later, the immunomodulation of digested samples it is evaluated means of cellular bioassay in Raw 264.7 and THP1 cells. Gene expression of pro-inflammatory biomarkers is analysed such iNOs, TNF or COX2 by rt-PCR. Preliminary results suggested that dialyzable fraction of food and food ingredients are able to modulate the gene expression of pro-inflammatory biomarkers. These results support the usefulness of the in vitro system gastrointestinal digestion-cellular bioassay as a rapid and cost-effective model for screening the bioavailability and immunomodulator properties of bioactive compounds present in food matrix.

\section{P069 \\ Moderate-Carbohydrate Diet Induces Changes in Leukocyte Gene Expression Patterns and Pro-Inflammatory Biomarkers Towards a More Healthy Phenotype, Compared to a High-Carbohydrate Diet}

\author{
Hans-Richard Brattbakk1, Ingerid Arbo 1, Mette Langaas', \\ Bård Kulseng ${ }^{2}$, Fedon Lindberg ${ }^{3}$, Berit Johansen ${ }^{1}$ \\ ${ }^{1}$ Norwegian University of Science and Technology- \\ Trondheim, Norway, ${ }^{2}$ St. Olavs Hospital, ${ }^{3}$ Dr. Fedon \\ Lindberg's Clinic, Norway
}

Introduction: Inflammation is associated with lifestyle diseases, including cardiovascular disease, type 2 diabetes and obesity. Diet is a central aspect of lifestyle, and food quality and quantity are important regulators of inflammatory mediators. DNA polymorphisms affect an individual's specific risks of disease onset which could be explained by genotype specific gene expression. Our objective was to determine diet specific differences in response to a moderate-carbohydrate diet compared to a high-carbohydrate diet.

Methods: In a randomized cross-over study, 32 slightly overweight subjects went through a two times six-day iso- and normocaloric diet intervention. Liquid meal replacement "smoothies" were designed composed of 65:15:20 and 27:30:43 energy percent of carbohydrates, proteins and fats, respectively. Common to both diets were a frequent eating pattern of 6 meals per day, vitamins/minerals, and equal quantity/quality of polyunsaturated fatty acids (PUFAs). Fasting blood samples for transcriptome, lipid and inflammatory biomarker analyses were collected before and after each diet period. Gene expression profiling was carried out using Illumina HT12 microarray. The subjects were genotyped for 66117 SNPs associated with metabolic and atherosclerotic-cardiovascular endpoints using the "Cardio-Metabochip", a custom Illumina iSelect genotyping array.

Results: A beneficial effect of dieting was observed. This was partly counteracted by the high-carbohydrate diet, while enforced by the moderate-carbohydrate diet. The latter was visualized by improved HOMA indices, statistical significant reduction in blood proinflammatory cytokine expression and improved blood lipid profiles compared to the high-carbohydrate diet. Transcriptome changes of several canonical pathways involved in immunological, inflammatory, proliferative, and apoptotic cell signaling was observed. Interindividual gene expression variability could be correlated to the presence of SNPs, thus, allowing for identification of eQTLs associated with metabolic traits.

Conclusions: A frequent meal eating pattern, sufficient PUFAs and a moderate-carbohydrate diet down regulate plasma biomarkers and leukocyte gene expression pathways associated with the onset and maintenance of lifestyle diseases. 


\section{P070 \\ 1,25 Dihydroxyvitamin D3 Down-regulated Inflammatory Markers Expression in Adipocytes}

\author{
Jean-François Landrier, Julie Marcotorchino, \\ Erwan Gouranton, Béatrice Romier, Christiane Malezet, \\ Marie-Josèphe Amiot \\ UMR 1260 Inra-Marseille, France
}

Since adipose tissue is characterized by a low grade inflammatory state during obesity, we aim to evaluate the impact of vitamin D3 on proinflammatory cytokine expression by adipocytes and adipose tissue. In 3T3-L1 adipocytes, a decrease of (mRNA and protein) was observed after a $24 \mathrm{~h}$ incubation with 1,25 dihydroxyvitamin D3 (VD3), according a dose response effect. VD3 was also able to reverse the proinflammatory effect of TNF. These results have been reproduced in 3T3-F442A, in human adipocytes and in mice adipose tissue explants ex vivo. The molecular mechanism has been studied. RNA interference experiences showed the role of the vitamin D Receptor in this regulation. However, no response element was identified in the human proximal promoters of Il6, I $\beta$ and MCP1, suggesting thus an indirect mechanism. The involvement of NF- $\mathrm{KB}$ signaling pathway has been demonstrated (decrease of IKK phosphorylation under VD3 effect). In parallel, we have shown an inhibition of $\mathrm{p} 38$ phosphorylation under VD3 effect, which could be related to the increase of Dusp10 expression, a phosphatase involved in p38 dephsophorylation. Together these molecular mechanisms allowed to explain at least in part the decrease of expression of the proinflammatory cytokines observed.

\section{P071 \\ Lycopene Inhibits Proinflammatorycytokine and Chemokine Expression in Adipose Tissue}

\section{Jean-François Landrier, Erwan Gouranton, \\ Clémentine Thabuis, Céline Riollet, Christiane Malezet, \\ Claire Malezet, Marie-Josèphe Amiot}

UMR 1260 Inra-Marseille, France

Obesity is associated with a low-grade inflammation which is correlated with an increased secretion of pro-inflammatory cytokines and chemokines by adipose tissue, suspected to contribute to the development of insulin resistance. Because lycopene is mostly stored in adipose tissue and possesses anti-inflammatory properties, we hypothesize that lycopene could reduce the production of proinflammatory markers in adipose tissue. In agreement with this hypothesis, we observed a decrease of inflammatory markers such as IL-6, MCP-1 and IL-1 at both the mRNA and protein level when explants of epididymal adipose tissue from mice fed with a high fat diet were incubated with lycopene ex vivo. The same effect was reproduced with explants of adipose tissue preincubated in lycopene and then subjected to TNF stimulation. The contribution of adipocytes and preadipocytes was evaluated. In both preadipocytes and differentiated 3T3-L1 adipocytes, lycopene preincubation for $24 \mathrm{~h}$ decreased the
TNF mediated induction of IL-6 and MCP-1. Finally, the same results were reproduced with human adipocyte primary cultures. The molecular mechanism was also studied. In transient transfections, a decrease of the luciferase gene reporter under control of NF-B responsive element was observed for cells incubated in the presence of lycopene and TNF compared to TNF alone. The involvement of the NF-B pathway was confirmed by the modulation of $\mathrm{IKK} / \mathrm{b}$ phosphorylation by lycopene. Altogether, these results showed for the first time a limiting effect of lycopene on adipose tissue proinflammatory cytokine and chemokine production. Such an effect could prevent or limit the prevalence of obesity-associated pathologies, such as insulin resistance.

\section{P072 \\ Dietary Antioxidant Polyphenols Reduce Inflammatory Angiogenesis Through MMP-9 and COX-2 Inhibition: A Potentially Protective Mechanism in Atherosclerotic Vascular Disease and Cancer}

María Annunziata Carluccio', Egeria Scoditti', Nadia Calabriso', Marika Massaro', Andrea Tinelli ${ }^{2}$.

María Rosa Montinari ${ }^{3}$, Carlo Storelli ${ }^{3}$,

Raffaele De Caterina ${ }^{4}$

${ }^{1}$ C.N.R. Institute of Clinical Physiology, Pisa and Lecce, Italy, ${ }^{2}$ Vito Fazzi Hospital-Lecce, Italy, ${ }^{3}$ Department of Biological and Environmental Science and Technology (DISTEBA), University of Salento, Italy, ${ }^{4 " G}$. d"Annunzio" University and Center of Excellence on Aging-Chiei, Italy

Objectives: Antioxidant phytochemicals, including the polyphenols from olive oil oleuropein (OE) and hydroxytyrosol (HT), and red wine antioxidant resveratrol (RSV) and quercetin (Q), are associated with low prevalence of cardiovascular diseases and cancer. Angiogenesis is a key process both in cancer and atherosclerosis, and is tightly regulated by molecular mediators including reactive oxygen species (ROS), cyclooxygenase (COX)-2 and matrix metalloproteinases (MMPs). We studied the effects of olive oil and red wine antioxidants on the angiogenic potential in an in vitro model of angiogenesis, and explored underlying mechanisms.

Methods: Human macro- (from umbilical vein: HUVEC) and micro-(from the dermis: HMEC) vascular endothelial cells were treated with 1-100 £gmol/L HT, OE, RSV or Q for $1 \mathrm{~h}$ before stimulation with VEGF or PMA. We then tested the cell migration and tube formation in a wound healing and a Matrigel assays, respectively, as well as the pro-angiogenic COX-2 expression by Western analysis; the expression and activity of the proteolytic enzyme MMP-9, by ELISA and zymography, respectively. We also assessed ROS intracellular levels by a DCF method.

Results: All dietary antioxidants tested reduced tube formation and endothelial cell migration after PMA stimulation, with an IC50 of $50 \mu \mathrm{mol} / \mathrm{L}$ for HT and OE and $30 \mu \mathrm{mol} / \mathrm{L}$ for RES and Q. The reduced angiogenesis was associated with the inhibition of COX-2 expression (IC50 of $10 \mu \mathrm{mol} / \mathrm{L}$ for HT and RES), MMP-9 release (IC50 of 50 $\mu \mathrm{mol} / \mathrm{L}$ for $\mathrm{HT}$ and $30 \mu \mathrm{mol} / \mathrm{L}$ for RES) and intracellular ROS levels (IC50 of $50 \mu \mathrm{mol} / \mathrm{L}$ for HT and $30 \mu \mathrm{mol} / \mathrm{L}$ for RES). Similar results were obtained with VEGF as a stimulus. 
Conclusions: Olive oil and red wine antioxidant polyphenols reduce inflammatory angiogenesis in vitro models of angiogenesis through MMP-9 and COX-2 inhibition. Our results suggest a potential protective role of dietary antioxidant polyphenols in atherosclerotic vascular disease and cancer.

\section{Obesity}

\section{P073 \\ Effects of Resveratrol and Pteroestilbene on the Expression of ATGL and HSL, Two Key Enzymes in Lipolysis}

\author{
Arrate Lasa', Martina Schweiger², Itziar Churruca1, \\ Edurne Simón 1 , María del Puy Portillo \\ ${ }^{1}$ Faculty of Pharmacy Vitoria-Gasteiz, Spain, ${ }^{2}$ Institute of \\ Molecular Biosciences, Spain
}

Introduction: Resveratrol (RSV) and pterostilbene are present in red wine. Resveratrol reduces some lipogenic gene expression, but its effect on lipolysis, has not been analyzed. Little is known about pterostilbene and its potential role in this catabolic pathway.

Methods: On day 12 mature SGBS adipocytes were treated with $100 \mu \mathrm{M}$ of trans-RSV or pterostilbene. After 24 hours, RNA was isolated for Hormone Sensitive Lipase (HSL) and Adipose Triglyceride Lipase (ATGL) expression analysis by Real Time RT-PCR. Epididymal adipose tissue extracts from WT, ATGL KO and HSL KO mice were incubated in the presence or absence of these 2 molecules $(100 \mu \mathrm{M})$ during 4 hours. FFA and glycerol in the medium were quantified by spectrophotometry. Results were analyzed by Student $t$ test.

Results: $100 \mu \mathrm{M}$ resveratrol increased ATGL expression in SGBS adipocytes but HSL remained unchanged. It increased FFA release in WT and HSL KO mice but not in ATGL KO, and did not alter glycerol release. Contrarily, pterostilbene enhanced the expression of both lipases in SGBS adipocytes and also FFA and glycerol release in all tissues.

Conclusion: Whereas resveratrol stimulates the first step of lipolysis, acting on ATGL, pterostilbene activates both lipases and thus, it stimulates the whole lipolytic reaction.

\section{P074 \\ Effects of Resveratrol on the Expression of Genes Related to Lipolysis in White Adipose Tissue}

\author{
Goiuri Alberdi Aresti, Jonatan Miranda, \\ Víctor Manuel Rodríguez, María Teresa Macarulla, \\ María Puy Portillo \\ Universidad del País Vasco, Vitoria-Gasteiz, Spain
}

Introduction: Resveratrol belongs to the family of polyphenols found in different plant species. The most common sources from diet include grapes, derived wine, peanut, nuts and berries. In the last years, research has demonstrated a variety of biological activities of resveratrol including cardio-protective, anti-carcinogenic, antiinflammatory and antioxidant activities as well as anti-obesity effects. The aim of the present work was to analyze the expression of genes involved in lipolysis.

Methods: Sixteen male Sprague-Dawley rats were divided into 2 experimental groups fed an obesogenic diet during 6 weeks: a control group (C) and an experimental group receiving a dose of $30 \mathrm{mg} / \mathrm{kg}$ body weight/day of resveratrol. Main adipose tissue depots (epidydimal, perirenal, mesenteric and subcutaneous) were dissected and weighed, and the Adipose Index was calculated as $\Sigma$ fat pad weights/ body weight. Expressions of perilipin (a lipid droplet associated protein that protects the adiposity), adipose triglyceride lipase (ATGL) and hormone sensitive lipase (HSL) were measured by RT-PCR in the epidydimal adipose tissue.

Results: Adipose Index was significantly reduced in the treated group in compared with the control group. Accordingly, HSL expression in the adipose tissue was significantly higher in this group $(\mathrm{P}<0.05)$. Neither the expression of ATGL nor perilipin were changed within the groups.

Conclusion: Resveratrol reduces fat mass, suggesting that it could act as anti-obesity agent. It can be proposed that is effect is due, at least in part, to enhanced lipolysis, as suggested by the increase in HSL expression. 


\section{P075}

\section{Site-Specific Circadian Expression of Leptin and Its Receptor in Human Adipose Tissue}

\author{
Purificación Gómez-Abellan', Cecilia Gómez-Santos ${ }^{1}$, \\ Juan Antonio Madrid', Fermín Ignacio Milagro", \\ Javier Campion², José Alfredo Martínez², \\ Juan Antonio Luján ${ }^{3}$, José María Ordovas ${ }^{4,5}$, \\ Marta Garaulet ${ }^{1,4}$ \\ ${ }^{1}$ Department of Physiology, Faculty of Biology, University \\ of Murcia, Spain, ${ }^{2}$ Department of Nutrition and Food \\ Sciences, Physiology and Toxicology, University of \\ Navarra-Pamplona, Spain, ${ }^{3}$ General Surgery Service, \\ University Hospital Virgen de la Arrixaca-Murcia, Spain, \\ ${ }^{4}$ Nutrition and Genomics Laboratory. Jean Mayer US \\ Deparment of Agriculture Human Nutrition Research \\ Center on Aging, at Tufts University-Boston, MA, \\ ${ }^{5}$ Department of Epidemiology, Centro Nacional \\ Investigaciones Cardiovasculares (CNIC)-Madrid, Spain
}

Brief Introduction: Circadian variability of circulating leptin levels has been well established over the last decade. However, the circadian behaviour of leptin in human adipose tissue remains unknown. This also applies to the soluble leptin receptor. Therefore, we investigated the ex vivo circadian behaviour of leptin and its receptor expression in human adipose tissue (AT).

Subjects and Methods: Visceral and subcutaneous abdominal AT biopsies ( $n=6)$ were obtained from morbid obese women (BMI $\geq 40$ $\mathrm{kg} / \mathrm{m}^{2}$ ). Anthropometric variables and fasting plasma glucose, lipids and lipoprotein concentrations were determined. In order to investigate rhythmic expression pattern of leptin and its receptor, AT explants were cultured during 24-h and gene expression was analyzed at the following times: 08:00, 14:00, 20:00, 02:00 h, using quantitative realtime PCR.

Results: Leptin expression showed an oscillatory pattern that was consistent with circadian rhythm in cultured AT. Similar patterns were noted for the leptin receptor. Leptin showed its achrophase (maximum expression) during the night, which might be associated to a lower degree of fat accumulation and higher mobilization. When comparing both fat depots, visceral AT anticipated its expression towards afternoon and evening hours.

Conclusions: Circadian rhythmicity has been demonstrated in leptin and its receptor in human AT cultures in a site-specific manner. This new knowledge paves the way for a better understanding of the autocrine/paracrine role of leptin in human AT.

\section{P076}

Temporal Order in Endogenous Circadian Rhythms of Genes Implicated in Human Adipose Tissue Metabolism

\author{
Marta Garaulet ${ }^{1,2}$, José María Ordovas ${ }^{2,3}$, \\ Purificación Gómez-Abellan', José Alfredo Martínez", \\ Juan Antonio Madrid ${ }^{1}$ \\ 'Department of Physiology, Faculty of Biology, University \\ of Murcia, Spain, 2Jean Mayer US Department of \\ Agriculture Human Nutrition Research Center on Aging, \\ Tufts University School of Medicine-Boston, MA, ${ }^{3}$ The \\ Department of Epidemiology and Population Genetics \\ Centro Nacional Investigación Cardiovascular (CNIC)- \\ Madrid, Spain, ${ }^{4}$ Department of Food Sciences, Physiology \\ and Toxicology, University of Navarra-Pamplona, Spain
}

Brief Introduction: Although it is well established that human adipose tissue (AT) shows circadian rhythmicity, published studies have been discussed as if tissues or systems showed only one or few circadian rhythms at a time. To provide an overall view of the internal temporal order of circadian rhythms in human AT including genes implicated in metabolic processes such as energy intake and expenditure, insulin resistance, adipocyte differentiation, dyslipidemia, and body fat distribution.

Methods: Visceral and subcutaneous abdominal AT biopsies $(\mathrm{n}=6)$ were obtained from morbid obese women $\left(\mathrm{BMI} \geq 40 \mathrm{~kg} / \mathrm{m}^{2}\right)$. To investigate rhythmic expression pattern, AT explants were cultured during 24-h and gene expression was analyzed at the following times: 0800, 1400, 2000, and $0200 \mathrm{~h}$ using quantitative real-time PCR. Clock genes, glucocorticoid metabolism-related genes, Leptin, Adiponectin and their receptors were studied.

Results: Significant differences were found both in achrophases and relative-amplitude among genes $(\mathrm{P}<0.05)$. Amplitude of most genes rhythms was high $(>30 \%)$. When interpreting the phase map of gene expression in both depots, data indicated that circadian rhythmicity of the genes studied followed a predictable physiological pattern, particularly for subcutaneous AT. Interesting are the relationships between adiponectin, leptin and glucocorticoid metabolism-related genes circadian profiles. Their metabolic significance is discussed. Visceral AT behaved in a different way than subcutaneous for most of the genes studied. For every gene, protein mRNA levels fluctuated during the day in synchrony with its receptors.

Conclusion: We have provided an overall view of the internal temporal order of circadian rhythms in human adipose tissue. 


\section{P077}

Effect of Trans Fatty Acids on Lipogenic and Oxidative Enzyme Expression in Liver of Mice Fed an Olive Oil Enriched Diet

\author{
Arrate Lasa', Juliana Sain², Marcela González², \\ Claudio Bernal', María del Puy Portillo ${ }^{1}$ \\ ${ }^{1}$ Universidad del País Vasco-Vitoria-Gasteiz, Spain, \\ ¿Universidad Nacional del Litoral, Argentina
}

Introduction: Trans fatty acid (TFA) intake is a matter of concern in some dietary patterns because of their negative effect on health. Thus, it is well known that these fatty acids increase LDLcholesterol and decrease HDL-cholesterol. However, their effects on lipid metabolism in liver have not been widely analysed.

Methods: CF1 male mice (22g) were fed for 30 days with a standard diet enriched with olive oil (7\%). This diet was supplemented (Olive + TFA) or not (Olive) with 1\% TFA. Liver was dissected and weighed. Lipids were extracted by Folch method and triacylglycerol (TG) content was measured spectrophotometrically by using a commercial kit. The expression of lipogenic enzymes (malic enzyme (EM), acetyl CoA carboxylase (ACC) and fatty acid synthase (FAS)), oxidative enzymes (carnitine palmitoyl transferase I (CPT-I) and acyl-CoA oxidase (ACO)), as well as that of their transcription factors (SREBP-1a, SREBP-1c and PPARá), were determined by Real Time RT-PCR. Results were analyzed by Student $t$ test.

Results: The inclusion of TFA in the diet did not modify liver weight, but it increased liver TG content. ACC, FAS and SREBP1a expression was significantly increased by TFA supplementation. In contrast, no changes were found in oxidative enzymes or PPARá expression.

Conclusion: The addition of TFA to an olive oil enriched diet modifies lipid metabolism in liver in mice. It increases liver fat content, at least in part, by enhancing lipogenesis, without changes in fatty acid oxidation.

\section{P078 \\ Leptin-Melanocortin Genes and Metabolic Traits in South African Learners}

\section{Yandi Yako', Boitumelo Fanampe', Mogamat Hassan², Lize van der Merwe ${ }^{3,4}$, Rajiv Erasmus ${ }^{1}$, Tandi Matsha ${ }^{2}$ \\ ${ }^{1}$ Stellenbosch University-Cape Town, South Africa, ${ }^{2}$ Cape Peninsula University of Technology, ${ }^{3} \mathrm{MRC}$, ${ }^{4}$ University of Western Cape, South Africa}

Background: Obesity is a multifactorial disease that is rarely associated with mutations in genes of the leptin-melanocortin pathway. Due to their direct cause-effect relationships with morbid obesity, these genetic defects are relevant in the study of the mechanisms underlying the common polygenic obesity. The present study investigated the contribution of the leptin-melanocortin genes in the development of obesity in South African children and adolescents.

Methods: School learners (110 Mixed Ancestry and 79 Black Africans) were screened for the presence of CART, GHRL, LEP and LEPR polymorphisms by sequencing PCR amplified gene fragments.
Genotype/allele association analysis was conducted to determine the effect of identified polymorphisms on the development of obesity.

Results: The following polymorphisms were detected LEP (19A>G, Lys36Arg, and Val94Met); LEPR (Lys109Arg, Gln223Arg, and Lys656Asn); GHRL (Leu72Met); CART (g.1281G>A, c.499delA, and g.517A $>\mathrm{G}$ ). In an optimal model containing all polymorphisms, age, gender, and ethnicity, learners with the LEPR-Lys109Arg g allele had 0.51 odd ratio of being obese than the major allele carriers. The minor alleles (c and g) of the LEPR-Lys656Asn and CART-g.517A $>\mathrm{G}$ were associated with increased obesity (odd ratio $=1.77$ and 5.98, respectively than the major allele carriers). After adjusting for age, gender and ethnicity, LEPR3-Lys656Asn and CART3-g.517A $>\mathrm{G}$ were individually (1.94 and 3.80 odd ratio, respectively) significantly associated with increasing obesity, while the LEPR-109Arg lowered the waist circumference.

Conclusion: The present study identified polymorphisms in the leptin-melanocortin genes and their association with obesity (body mass index and waist circumference, confirming the contribution of more than one polymorphisms in weight regulation.

\section{P079 \\ Prevalence of Metabolic Syndrome Associated with Glucose Tolerance and Body Mass Index (BMI) in a Mixed Ancestry Population of South Africa}

\author{
Zelda Vergotine1, M. Hassen ${ }^{2}$, R.T. Erasmus ${ }^{3}$, T. Matsha ${ }^{4}$ \\ ${ }^{1}$ Faculty of Health and Wellness Sciences, Cape Peninsula \\ University of Technology, ${ }^{2}$ Department Nursing/Radiology, \\ Cape Peninsula University of Technology, ${ }^{3}$ Division \\ Chemical Pathology, NHLS, Stellenbosch University, \\ ${ }^{4}$ Department Biomedical Sciences, Cape Peninsula \\ University of Technology, South Africa
}

Introduction: Metabolic Syndrome (MetS) is a constellation of metabolic disturbances, with the pathophysiology largely attributable to insulin resistance or Syndrome $\mathrm{X}$ with the major contributor for development being an overabundance of circulating fatty acids derived mainly from adipose tissue affecting the insulin-mediated glucose transport and lipid metabolism. Over the past two decades the incidence of obesity and diabetes mellitus (DM) has dramatically increased globally with the multi-racial population of South Africa being no exception.

Objective: Determine the prevalence of the metabolic syndrome and investigate its association with glucose tolerance and body mass index (BMI) in a mixed ancestry population from Cape Town, South Africa.

Methods: Complete data, including oral glucose tolerance tests of 904 subjects between the ages 16-95 years participated in this study. Metabolic syndrome was defined according to NCEP ATP III criteria (2001) and WHO criteria (1999) for diabetes.

Results: Prevalence of MetS was found to be 39\% (351) and 254 subjects (including 115 self-reported) had diabetes. In 466 (52\%) of subjects had normal glucose tolerance with only 17 (3.65\%) of subjects had metabolic syndrome. Whilst in DM, impaired fasting glucose (IFG) and impaired glucose tolerance (IGT) subjects, MetS was respectively, $39 \%, 23.5 \%$ and $12 \%$. As for the BMI of subjects: IGT 
$\left(30.8 \mathrm{~kg} / \mathrm{m}^{2}\right)$ and DM $\left(31.6 \mathrm{~kg} / \mathrm{m}^{2}\right)$ were significantly higher than the normoglycaemic $\left(28.6 \mathrm{~kg} / \mathrm{m}^{2}\right)$ subjects. No significant differences were observed between subjects with IFG $\left(30.4 \mathrm{~kg} / \mathrm{m}^{2}\right)$, normoglycaemia and diabetes.

Conclusion: There is a high prevalence of the metabolic syndrome with the components including obesity and abnormal glucose intolerance in the mixed ancestry population of South Africa. The elevated risk not only of diabetes but also cardiovascular diseases highlights the adverse effects of MetS and the urgent need for strategies to prevent the emerging epidemic in the near future within this population.

P080

Role of $\beta 2$-adrenergic Receptor

Polymorphisms (GIn27Glu and Arg16Gly) on Body Weight and Body Composition Response to Energy Restriction in Spanish Obese Women

Jonatan Ruiz', Eider Larrarte², Javier Margareto², Francisco Ortega1, Raquel Ares ${ }^{3}$, Idoia Labayen ${ }^{3}$

${ }^{1}$ Karolinska Institutet, Sweden, ${ }^{2}$ LEIA Foundation,

${ }^{3}$ University of the Basque Country, Vitoria, Spain

We investigated the role of common $\beta 2$-adrenergic receptor (ADRB2) rs1042714 (Gln27Glu) and rs1042713 (Arg16Gly) polymorphisms on body weight and body composition response to 12 -week energy-restricted diet in women. The study comprised 78 Spanish obese [body mass index (BMI): $34.0 \pm 2.8 \mathrm{~kg} / \mathrm{m}^{2}$ ] women (age: $36.7 \pm 7 y$ ). We measured (before and after the dietary intervention) weight and height, and BMI calculated. Moreover, body fat mass (FM) and lean mass (LM) were measured by Dual Energy X-ray Absorptiometry. We observed an interaction effect between the Gln27Glu polymorphism and diet-induced changes on body weight $(P=0.006)$, BMI $(P=0.004)$, and LM $(P=0.001)$. Women carrying the Glu allele had a greater reduction in body weight than non-Glu allele carriers ( $9.5 \pm 2.9$ vs. $7.0 \pm 3.5 \%$, respectively, $P=0.002$ ). Moreover, women with the Glu allele lost more LM than the Gln27Gln group (5.9 \pm 2.7 vs. $4.0 \pm 2.7 \%$, respectively, $P=0.001$ ). We did not find any significant interaction effect between the Arg16Gly polymorphism and diet-induced changes on the outcome variables (all $P>0.1$ ). The results suggest that the $A D R B 2$ Gln27Glu polymorphism has a modulating effect on diet-induced changes on body weight and body composition, and should be considered in future obesity treatments. These findings should taken as preliminary and be replicated in further energy restriction studies with larger sample sizes.
P081

The Effect of Ponderal Index at Birth on the Relationships Between Common LEP and LEPR Polymorphisms and Adiposity in Adolescents; The Helena Study

Idoia Labayen ${ }^{1}$, Jonatan Ruiz², Luis Moreno ${ }^{3}$, Francisco Ortega ${ }^{2}$, Laurent Beghin', Stefaan DeHenauw ${ }^{5}$, Pedro Benito6, Ligia Díaz', Marika Ferrari", George Moschonis ${ }^{9}$

${ }^{1}$ University of The Basque Country-Vitoria, Spain, ${ }^{2}$ Karolinska Institutet, ${ }^{3}$ University of Zaragoza, ${ }^{4}$ INSERMFrance, ${ }^{5}$ University of Ghent, ${ }^{6}$ Universidad Politécnica de Madrid, ${ }^{7}$ CSIC-Madrid, ${ }^{8}$ INRAN-Roma, ${ }^{9}$ University of Athens, Greece

Objective: To examine the effect of pondered index (PI) at birth on the relationships between eight common polymorphims of the LEP and LEPR genes and adiposity in adolescents.

Methods: A total of 823 European adolescents (45.4\% girls) aged 14.8,,b1.4 years from the HELENA study were genotyped for the LEP (rs2167270, rs12706832, rs10244320, rs2071045 and rs3828942) and LEPR (rs1137100, rs1137101 and rs8179183) polymorphisms. The PI was calculated from parental reports of birth weight and length. The body mass index (BMI) and fat mass index (FMI) were calculated. Analyses were adjusted for relevant confounders.

Results: The LEP rs10244329 and rs3828942 polymorphisms were associated with higher FMI only in adolescents within the lower PI tertile $\left(+0.55 \mathrm{~kg} / \mathrm{m}^{2}\right.$ per minor $\mathrm{T}$ allele, adjusted $\mathrm{P}=0.040$, and $+0.58 \mathrm{~kg} / \mathrm{m}^{2}$ per major $\mathrm{G}$ allele, adjusted $\mathrm{P}=0.028$, for $\mathrm{rs} 10244329$ and rs3828942, respectively). Similarly, the LEPR rs8179183 polymorphism was significantly associated with higher FMI in adolescents within the lower PI tertile $\left(+0.87 \mathrm{~kg} / \mathrm{m}^{2}\right.$ per minor $\mathrm{C}$ allele, adjusted $\mathrm{P}=0.006$ ).

Conclusions: Ponderal index at birth, a proxy of newborn nutritional status, interact with the LEP rs10244329 and rs3828942 polymorphisms and the LEPR rs8179183 polymorphisms leading to higher susceptibility to the deleterious effect of risk alleles on total adiposity in those adolescents born with low PI.

\section{P082}

FABP2 Ala54Thr Polymorphism: Association with Biochemical Markers and Insulin Resistance in Obese Mexican Children

Zacarias Jiménez-Salas, Eduardo Campos-Góngora, Blanca Edelia González-Martínez, Elizabeth Solís-Pérez, Manuel López-Cabanillas, Armando Quintanilla-Cantú

Universidad Autónoma de Nuevo León-Monterrey, México

Introduction: Several researches show that people with obesity have an increase risk of cardiovascular diseases; previous studies suggest that the development of the disease also could be influenced by a mutation in the FABP2 gene. The fatty acid protein binding (FABP2) an intracellular component expressed in the intestine that 
puts together lipids. The variant of the FABP2 Ala54Thr has altered the role and has been associated with insulin resistance and obesity.

Objective: Assess the association of FABP2 Ala54Thr polymorphism with biochemical and anthropometric variables in Mexican childhood obesity.

Methods: We included children from 6 to 12 years old attending in the Endocrinology Clinic of Jose Eleuterio Gonzalez/UANL Hospital. The subjects were separated in two groups: 34 with $\mathrm{BMI}>85 \mathrm{P}^{\circ}$ and 20 with $\mathrm{BMI}<84 \mathrm{P}^{\circ}$ as a control. Peripheral blood samples were collected to measure the lipid profile; insulin resistance was determined by HOMA method. The polymorphism was analyzed by RFLP-PCR. All data obtained was analyzed using the SPSS version 17.0.

Results: The subjects with $\mathrm{BMI}>85 \mathrm{P}^{\circ}$ had higher prevalence of insulin resistance and serum levels of insulin, leptin and triglycerides than the control group. In both groups the polymorphism genotypes followed the expected distribution. The $\mathrm{BMI}>85 \mathrm{P}^{\circ}$ group showed 58 subjects with phenotype Ala/Ala, 6 with Thr/Thr and 25 with Ala/Thr. Glucose and cholesterol levels were not statistically different between groups $(\mathrm{p}=0.3$ and $\mathrm{p}=0.09$ ). No significant differences were found according to genotype, analyzing the biochemical and anthropometric variables in the group with higher BMI $\left(\mathrm{BMI}>85 \mathrm{P}^{\circ}\right)$. It was found OR of 2.0 for genotype Ala/Ala versus Thr/Thr and Ala/Thr in both groups.

Conclusion: No significant associations of the polymorphism were found with obesity, although the study suggests the genotype TT is a risk factor for obesity development in Mexican childhood. We suggest increasing the size of the sample to corroborate these findings.

\section{P083 \\ MTHFR C677T Polymorphism: Association with Biochemical Markers and Insulin Resistance in Obese Mexican Children}

Zacarias Jiménez-Salas, Eduardo Campos-Góngora, Blanca Edelia González-Martínez, Elizabeth Solís-Pérez, Manuel López-Cabanillas, Reyna Esmeralda CalderónEnríquez

Universidad Autónoma de Nuevo León-Monterrey, México

Introduction: Several researches show that people with obesity have an increase risk of cardiovascular diseases; previous studies suggest that the development of the disease also could be influenced by a mutation in the MTHFR gene. 5, 10- Methylenetetrahydrofolate reductase (MTHFR) is an enzyme associated with serum homocysteine levels. A decrease in the activity of this enzyme caused by a mutation in the MTHFR gene C677T increases cardiovascular risk.

Objective: Assess the association of MTHFR C677T polymorphism with biochemical and anthropometric variables in Mexican childhood obesity.

Methods: We included children from 6 to 12 years old attending in the Endocrinology Clinic of Jose Eleuterio Gonzalez/UANL Hospital. The subjects were separated in two groups: 34 with $\mathrm{BMI}>85 \mathrm{P}^{\circ}$ and 20 with $\mathrm{BMI}<84 \mathrm{P}^{\circ}$ as a control. Peripheral blood samples were collected to measure the lipid profile; insulin resistance was determined by HOMA method. The polymorphism was analyzed by RFLP-PCR. All data obtained was analyzed using the SPSS version 17.0.

Results: The subjects with $\mathrm{BMI}>85 \mathrm{P}^{\circ}$ had higher prevalence of insulin resistance and serum levels of insulin, leptin and triglycerides than the control group. In both groups the polymorphism genotypes followed the expected distribution. The $\mathrm{BMI}>85 \mathrm{P}^{\circ}$ group showed 7 subjects with genotype CC, 11 with TT and 16 with TC. Glucose and cholesterol levels were not statistically different between groups ( $\mathrm{p}=0.3$ and $\mathrm{p}=0.09$ ). No significant differences were found according to genotype, analyzing the biochemical and anthropometric variables in the group with higher $\mathrm{BMI}\left(\mathrm{BMI}>85 \mathrm{P}^{\circ}\right)$. It was found $\mathrm{OR}$ of 3.95 for genotype TT versus $\mathrm{CC}$ and $\mathrm{CT}$ in both groups.

Conclusion: No significant associations of the polymorphism were found with obesity, although the study suggests the genotype TT is a risk factor for obesity development in Mexican childhood. We suggest increasing the size of the sample to corroborate these findings.

\section{P084}

\section{Allelic Variants of the Melanocortin-3 Receptor Gene (MC3R) and Weight Loss in Obesity: Interaction with Hypo-energetic High-fat Versus Low-fat Diets}

José L. Santos ${ }^{1,2}$ J. Alfredo Martínez², Thorkild IA Sorensen ${ }^{3}$, Rolando De La Cruz ${ }^{4}$, Claus Holst ${ }^{3}$, Katrine Grau ${ }^{3}$, Carolina Naranjo 4 , Alberto Maiz², Arne Astrup ${ }^{5}$, Wim HM Saris', lan McDonald ${ }^{8}$, JeanMichel Oppert' ${ }^{7}$ Torben Hansen ${ }^{9}$, Oluf Pedersen ${ }^{9}$, NUGENOB Consortium ${ }^{10}$

${ }^{1}$ Department of Physiology and Nutrition, University of Navarra, Pamplona, Spain, ${ }^{2}$ Department of Nutrition, Diabetes and Metabolism. Pontificia Universidad Católica de Chile, Santiago, Chile, ${ }^{3}$ Institute of Preventive Medicine, Copenhagen University Hospital, Centre for Health and Society, Copenhagen, Denmark, ${ }^{4}$ Department of Public Health, School of Medicine, Pontificia Universidad Católica de Chile, Santiago, Chile, ${ }^{5}$ Department of Human Nutrition, Faculty of Life Science, University of Copenhagen, Copenhagen, Denmark, ${ }^{6}$ Department of Human Biology, Nutrition and Toxicology Research Institute Maastricht, Maastricht University, Maastricht, Netherlands, 7 Department of Nutrition, HotelDieu Hospital (AP-HP), University Pierre ET Marie CurieParis 6, Human Nutrition Research Center Ile-de-France, Paris, France, ${ }^{8}$ School of Biomedical Science, University of Nottingham Medical School, Queen's Medical Centre, Nottingham, United Kingdom, ${ }^{9}$ Hagedorn Research Institute, Gentofte, Denmark, ${ }^{10}$ Institute of Biomedical science, University of Copenhagen, Copenhagen, Denmark

Introduction: The melanocortin system plays an important role in energy homeostasis. In humans, Thr6Lys and Val81Ile variants of the melanocortin-3 receptor gene (MC3R) have been associated with childhood obesity, higher BMI Z-score, elevated body fat percentage and reduced fat oxidation compared to non-carriers. Based on studies 
supporting the participation of MC3R variants in weight loss responses in obese children, the aim of this study is to assess the association between several allelic variants of MC3R with weight loss induced by energy-restricted diets in obese European adults.

Subjects and Methods: This research is based on the NUGENOB study, a randomised, parallel, two-arm, open-label multicentre trial conducted to assess weight loss during a 10-week dietary intervention involving two different hypo-energetic (-600 kcal/day) diets with a targeted fat energy of $20 \%-25 \%$ or $40 \%-45 \%$. This trial involved 760 participants genotyped for 10 single nucleotide polymorphisms covering the single exon of MC3R gene and its flanking regions, including the missense variants Thr6Ile and Val81Ile. Linear mixed models and haplotype-based analysis were carried out to assess the potential association between genetic polymorphisms with differential weight loss, fat mass loss and waist change as well as gene*diet interactions.

Results: In the multivariate analysis, only rs6014646 was significantly associated with weight loss using the co-dominant $(\mathrm{p}=0.04)$ and dominant models $(\mathrm{p}=0.03)$. These $\mathrm{p}$-values were not statistically significant after control for multiple testing. Haplotype based multivariate analysis using permutations showed that rs3827103-rs 1543873 $(\mathrm{p}=0.06)$, rs6014646-rs6024730 $(\mathrm{p}=0.05)$ and $\mathrm{rs} 3746619-\mathrm{rs} 3827103$ $(\mathrm{p}=0.10)$ displayed near-statistical significant results in relation to weight loss. No significant gene*diet interaction was found.

Conclusion: Although rs6014646 showed nominal statistical significance under 0.05 level in relation to weight loss, there is an overall insufficient evidence to support a major effect of genetic variants of MC3R and differential weight loss after a 10-week dietary intervention.

\section{P085}

\section{Association Between Chilhood Eating Behaviour, IL18 Genetic Markers and Obesity in a Case-Control Study in Chile}

\section{Andrea González, Susan V. Smalley, Judith Ho-Urriola, M. Isabel Hodgson, L. Rodrigo Cataldo, Daniela González, José L. Santos \\ Pontificia Universidad Católica de Chile-Santiago, Chile}

Introduction: Plasma levels of interleukin-18 (IL18) have been associated with the metabolic syndrome, insulin resistance and obesity. Mice genetically deficient in IL18 are hyperphagic and obese, suggesting that IL18 participates in eating behaviour. The aim of this study was to assess the influence of IL18 genetic markers in childhood eating behaviour scores and obesity.

Design and Methods: We conducted a case-control study in the city of Santiago (Chile) with a sample of 191 obese children (above 95th percentile of BMI NCHS/CDC 2000) and 119 normal weight (25-75 ${ }^{\text {th }}$ percentiles NCHS/CDC 2000; both genders; 6-12 years old). Genotypes of IL18 rs2043055 (-5848T >C), rs360729 (+8855T >A) and rs549908 $(+4860 \mathrm{~A}>\mathrm{C})$ were determined using PCR-RFLP techniques and Taqman assays. Eating behavior scores were calculated using the Child Eating Behavior Questionnaire (CEBQ) adapted for Chilean families. CEBQ showed Cronbach's alpha $>0.8$, while the structure of eight factors of the original CEBQ was replicated as shown by multivariate factorial analysis.
Results: Eating behavior scores were strongly associated with childhood obesity in Chilean children. Eating behaviour scores indicating positive inclinations to higher intake were strongly associated with childhood obesity $(\mathrm{p}<0.001)$ in the subscales "enjoyment of food", "emotional overeating" and "food responsiveness". Foodavoidant subscales "satiety responsiveness" and "slowness in eating" were also inversely associated with childhood obesity $(\mathrm{p}<0.001)$. Genotype frequencies do not significantly deviated from HardyWeinberg expectations. The IL18 genetic polymorphisms $-5848 \mathrm{~T}>\mathrm{C}$ showed a significant dose-response association with childhood obesity $(\mathrm{p}=0.01)$ (odds ratio for heterozygous genotype $=1.8 ; 95 \% \mathrm{CI}$ : $1.0-3.2$; odds ratio for homozygous alternative $=3.0$; $95 \%$ CI: 1.5 6.3; reference: homozygous wild-type). No significant associations were found between IL18 genotypes and eating behaviour scores.

Conclusion: Our study shows a strong association between specific eating behavior scores and childhood obesity. The association between IL18 rs2043055 genotypes with childhood obesity needs replication and further assessment with additional IL18 promoter polymorphisms.

\section{P086 \\ Changes of Lipid Metabolism During Development of Obesity in Dio Mice}

Hae-Jin Park, Su-Jung Cho, Eun-Young Kwon, SeonMin Jeon, Yong Bok Park, Myung-Sook Choi

Kyungpook National University-Daegu, Republic of Korea

Introduction: Dietary fat is one of the most important environmental factors associated with the occurrence of obesity. Although diet induced obese animals are considered a more comparable model for human obesity than genetically obese animals.

Methods and Materials: We investigated to identify the changes of related lipid metabolism biomarkers for high-fat diet (HFD, 20\% fat, $1 \%$ cholesterol) in C57BL/6J mice. The mice were sacrificed at 2, 4, 6, 8, 12, 16, 20 and 24 weeks in sequence and hepatic microarray was analysed.

Results and Conclusions: In the hepatic lipid synthesis related enzyme activities, glucose-6-phosphate dehydrogenase (G6PD) activity was significantly increased in the HFD group during entire experimental period compared to the ND group. The malic enzyme activity was significantly higher at only 2, 4, 6 and 8 weeks, and fatty acid synthase (FAS) enzyme activity was higher at 12 and 24 weeks in the HFD group than in the ND group. Of the hepatic lipid oxidation related enzymes, carnitine palmitoyl transferase (CPT) and beta-oxidation activities were significantly increased each weeks. Also, immunohistochemical staining for sterol regulatory element binding proteins (SREBP 1,2) and peroxisome proliferator-activated receptor $\gamma(\operatorname{PPAR} \gamma)$ in hepatocytes showed strong immunoreactivity by chronic HFD diet. The microarray was applied to acquire a further understanding of the HFD-induced transcriptional adaptations in liver tissue. So far, we have validated applicable biomarkers about lipid metabolism of diet-induced obesity. The early (fist 8 wks) down-regulated HFD-responsive genes were Acadvl, Acadsb, Acadm, Adh1, Acads, Ehhadh, Acadl, Acat1, Dci, and Cptla which are regulated to the fatty acid metabolism. The late (after $12 \mathrm{wks}$ ) down-regulated HFD-responsive genes were Mvd, Sqle, Lss, Pmvk and Idil which 
are related to the steroid biosynthesis. These results showed that HFD induced the alteration of hepatic genes related to the lipid metabolism in the early and late stage.

\section{P087 \\ Profile of Adipose Tissue Gene Expression in Pre-and Postmenopausal Women Site- Specific Differences}

\author{
Cecilia Gómez-Santos ${ }^{1}$, Juan José Hernandez-Morante ${ }^{1,2}$, \\ Javier Margareto ${ }^{3}$, Eider Larrarte $^{3}$, Xavier Formiguera $^{4}$, \\ Marta Garaulet ${ }^{1}$ \\ 1Department of Physiology University of Murcia, Spain, \\ ${ }^{2}$ School of Nursing Studies Catholic University of Murcia, \\ Spain, 'LEIA Foundation for Biomolecular Technology- \\ Vitoria, Spain, ${ }^{4}$ Morbid Obesity Unit, University Hospital \\ Germans i Pujol-Barcelona, Spain
}

Objectives: Menopause increases the risk of several pathologies, probably due to enlarged levels of visceral fat. Apart from morphological and endocrinal changes, a cluster of genes, still not fully defined, may be involved in these alterations. The objective of the present study, therefore, was to analyze differences in adipose tissue gene expression between pre- and post-menopausal women, and to ascertain whether any differences were depot-specific.

Methods: Visceral adipose tissue (VAT) and subcutaneous adipose tissue (SAT) biopsies were taken from 7 pre- and 7 postmenopausal women undergoing surgery due to morbid obesity. Adipose tissue RNA was extracted and, the overall gene expression profile was analysed by microarray analysis.

Results: In general, SAT genes were over expressed while VAT was down regulated in premenopausal compared with postmenopausal women. We found 724 differentially expressed genes in SAT and 327 in VAT. These differences suggest that several biological processes, such as those involved in the immune system and other metabolic processes were altered with menopausal status. As regards individual genes, Neurexin3 (NRXN3), Metallothionein1E (MT1E) and Keratyn7 (KRT7) showed the most pronounced differences. Interestingly, the expression of these genes was related to body fat distribution.

Conclusions: Our results reveal that menopause influences the adipose tissue expression of many genes, especially of NXRN3, MT1E and KRT7, which are associated with the alteration of several key biological processes. Gene expression in adipose tissue could be used for diagnosis and the development of new therapeutic strategies against obesity and related alterations, according to menopausal status.

\section{P088 \\ Regulation of Chemerin Secretion and Gene Expression by Insulin, Troglitazone and Free Fatty Acids in 3T3-L1 Adipocytes}

André Gustavo Vasconcellos Costa ${ }^{1,2}$,

Silvia Lorente Cebrián ${ }^{1}$, Pedro L. Prieto-Hontoria1,

Josefina Bressan³, J. Alfredo Martínez', María J. Moreno-

Aliaga ${ }^{1}$

${ }^{1}$ University of Navarra-Pamplona, Spain, ${ }^{2}$ Department of Food Technology, Federal University, ${ }^{3}$ Department of

Nutrition and Health, Federal University of Viçosa, Brazil

Introduction: Chemerin is novel adipokine involved in the regulation of adipocytes differentiation. Recently, it has been reported that serum chemerin levels are positively associated with several components of metabolic syndrome such as obesity, insulin resistance and type 2 diabetes. Eicosapentaenoic acid (EPA) and medium chain fatty acids (MCFA) have shown beneficial effects on obesity and insulin resistance. The aim of this study was to examine the effects of EPA and MCFA as well as insulin and PPAR agonist Troglitazone in chemerin protein secretion and gene expression levels in 3T3-L1 adipocytes.

Methods: Mature 3T3-L1 adipocytes were serum-starved for 3 hours and then incubated with the appropriate treatment $(10 \mu \mathrm{M}$ Troglitazone or 100-200 $\mu \mathrm{M}$ EPA or $250 \mu \mathrm{M} \mathrm{C8:0} \mathrm{or} 250 \mu \mathrm{M}$ C12:0) in presence or absence of insulin (100 nM) during 24 hours. Changes in chemerin mRNA expression were investigated using real-time RT-PCR and the amount of chemerin secreted into the media was measured by a specific ELISA kit.

Results: Insulin treatment produced a significant increase $(+69.5 \%, \mathrm{p}=0.01)$ in the total amount of chemerin secreted to the media, without altering chemerin mRNA levels in 3T3-L1 adipocytes. In contrast, treatment with Troglitazone, EPA and MCFA did not modify either basal or insulin-stimulated chemerin secretion. Furthermore, no changes on chemerin mRNA expression were observed in adipocytes treated with Troglitazone, EPA and MCFA.

Conclusion: These results suggest that insulin positively regulates chemerin secretion probably by posttranscriptional mechanisms. PPAR agonists and free fatty acids (EPA, C8:0 and C12:0) do not seem to be involved in the regulation of chemerin production in 3T3L1 adipocytes.

\section{P089 \\ Effects of Lipoic Acid on SP1 Activity in 3T3-L1 Adipocytes}
Pedro L. Prieto-Hontoria ${ }^{1}$, Patricia Pérez-Matute2, Marta Fernández-Galilea', J. Alfredo Martínez',
María J. Moreno-Aliaga'
${ }^{1}$ University of Navarra-Pamplona, Spain, ${ }^{2}$ HIV and Associated Metabolic Alterations Unit Infectious Disease Area. Center for Biomedical Research of La Rioja (CIBIR)- Logroña, Spain

Introduction: Lipoic Acid (LA) is a natural antioxidant compound that has shown beneficial effects in obesity and insulin resis-
106

J Nutrigenet Nutrigenomics 2010;3:49-124 4th Congress of the International Society of Nutrigenetics/Nutrigenomics (ISNN) 
tance. Previous studies have demonstrated that LA reduced the ability of adipocytes to produce leptin both in vitro and in vivo. Sp1 is a ubiquitously transcription factor that may function as a cellular glucose sensor. Furthermore, $\mathrm{Sp} 1$ is also a key factor involved in the regulation of leptin gene. Thus, the aim of this study was to analyze the effects of LA on Spl activity and its potential regulation by phosphorylation.

Methods: Nuclear extracts were prepared from fully differentiated 3T3-L1 adipocytes treated with LA $(250 \mathrm{M})$. Sp1 binding activity to DNA was determined using a Sp1 Transcription Factor ELISA kit (Panomics). Phosphorylation of Sp1 was analyzed by Western Blot using a phospho-Sp1 (Thr 453) antibody (Abcam).

Results: LA treatment down regulated leptin mRNA levels and decreased leptin secretion in a dose-dependent manner. Moreover, nuclear extracts from these LA-treated adipocytes for $24 \mathrm{~h}$ exhibited a decreased abundance of Sp1-DNA complexes $(\mathrm{P}<0.01)$. Our data showed that the phosphorylation of $\mathrm{Sp} 1$ in Thr453 was significantly increased $(\mathrm{P}<0.05)$ in LA-treated adipocytes. Moreover, treatment of nuclear extracts with protein phosphatase1 (PP1), an enzyme that dephosphorylates SP1, abolished the inhibitory effect of LA on Sp1 transcriptional activity.

Conclusion: Our data suggest that the phosphorylation of Sp1 and the reduced DNA-binding activity of this transcription factor could be involved in the inhibition of leptin induced by LA.

\section{P090 \\ Early Life Stress Modifies the Response to an Obesigenic Diet in the Adult State}

\section{Laura Paternain', Noemi Boqué1, Fermin I. Milagro', Eva Martisová2, Ma Javier Ramírez², J. Alfredo Martínez', Javier Campion ${ }^{1}$ \\ ${ }^{1}$ University of Navarra-Pamplona, Spain, ${ }^{2}$ Department of Pharmacology University of Navarra, Spain}

Introduction: Stressful early life environment has been postulated to be involved in the susceptibility to different diseases in later life such as mental disorders, cancer, diabetes mellitus and obesity.

Methods and Material: In this work we investigated the effects of a high-fat-sugar diet (HFS) intake for 35 days on adult female Wistar rats which had experienced $180 \mathrm{~min}$ daily separations from the dam (MS) during the first 3 weeks of life. After the sacrifice changes in the obesity phenotype (adiposity and serum leptin), glucocorticoid biomarker (serum corticosterone), insulin resistance biochemical profiles (insulin) and the expression of different genes related to obesity in periovaric adipose tissue were analyzed.

Results: HFS intake induced a statistically significant increase on body weight $(\mathrm{p}<0.05)$, adiposity $(\mathrm{p}<0.001)$ and serum leptin levels $(\mathrm{p}=0.001)$ while MS decreased fat pads mass but only on rats fed on HFS $(p<0.05)$. Concerning insulin resistance markers there were no changes due to the diet but MS reduced serum insulin levels $(p<0.01)$ and HOMA index $(\mathrm{p}<0.05)$ only on rats fed on a control diet. Corticosterone serum levels were reduced $(\mathrm{p}<0.05)$ in the animals that were subjected to maternal separation independently of the diet. Finally, multiple gene expression analysis revealed significant differences between HFS and HFS-MS group, especially for a set of lipolytic genes as Adiponutrin $(+135 \%, \mathrm{p}<0.05)$, Hormone-sensitive lipase $(+88 \%, \mathrm{p}<0.01)$ and Perilipin $(+94 \%, \mathrm{p}<0.01)$.
Conclusion: All these results revealed that a stressful situation during the neonatal state (maternal separation) alters the response to a high-fat-sugar diet in the later life. The possible mechanisms involved in this relationship could be through modifications of the insulin signaling on the glucocorticoid response and on the lipid metabolism in visceral adipose tissue.

\section{P091 \\ Sedentary Behavior Increases Obesity Risk Linked to the rs9939609 Polymorphism of the FTO Gene in a Spanish Children and Adolescents Case-control Study}

\author{
Adriana Moleres', Tara Rendo-Urteaga1, \\ $M^{a}$ del Carmen Ochoa ${ }^{2}$, Cecilia Galbete', \\ Miguel Ángel Martínez-González³, Cristina Azcona- \\ Sanjulian4, J. Alfredo Martínez', Amelia Marti ${ }^{1}$ \\ ${ }^{1}$ University of Navarra-Pamplona, Spain, ${ }^{2}$ Center for \\ Applied Medical Research, University of Navarra- \\ Pamplona, Spain, ${ }^{3}$ Department of Preventive Medicine \\ and Public Health University of Navarra-Pamplona, Spain, \\ ${ }^{4}$ Paediatric Endocrinology Unit Department of Paediatric, \\ Clinica Universidad de Navarra-Pamplona, Spain
}

Introduction: FTO rs9939609 polymorphism has recently been strongly associated with excessive body weight and adiposity. Some studies have shown the attenuation of the effect of this SNP on total and central body fat by physical activity in adolescents and have demonstrated that a low physical activity accentuates the effect of this polymorphism on body fat accumulation.

Subjects and Methods: The study population included 354 children and adolescents (6-18 years) enrolled in a case-control study in Navarra (Spain). Sedentary lifestyle was assessed through the number of hours spent watching TV or using computers, during school days and on weekends. Obesity was defined according to Spanish BMI reference data. Subjects were genotyped for the rs9939609 SNP of the FTO gene using real-time PCR (RT-PCR) followed by allelediscrimination.

Results: The distribution of genotypes for the rs9939609 polymorphism of the FTO gene variant was in Hardy-Weinberg equilibrium in the population with a minor A allele frequency of 0.46 . This $\mathrm{SNP}$ presents a strong association with obesity risk by itself $(\mathrm{OR}=2.15$, 95\% CI=1.13-4.10, $\mathrm{p}=0.019)$. Considering a dominant model and after adjusting for age and gender, children and adolescents who are carriers of the mutation and spent more than 14 hours/week watching TV or using computers had a threefold higher risk of developing obesity $(\mathrm{OR}=3.1,95 \% \mathrm{CI}=1.73-5.54, \mathrm{p}<0.001)$ than those with a less sedentary behaviour. Moreover, carriers of the A allele of the polymorphism had a $5.2 \%$ increase in obesity risk per hour of TV watching or computers use.

Conclusions: In the present study we confirm the relationship between sedentary behaviour and the rs 9939609 polymorphism of the FTO gene on obesity risk. Children and adolescents carriers of the mutation and spending more than 14 hours/week watching TV or using computers multiply by 3 their risk of becoming obese, compared with non-carrier subjects. 


\section{P092}

\section{Vitamin C Modulates the Interaction Between Adipocytes and Macrophages}

Diego García-Díaz, Javier Campion, Pablo Quintero, Fermín I. Milagro, María J. Moreno-Aliaga,

J. Alfredo Martínez

University of Navarra, Pamplona, Spain

Introduction: Increased body fat accumulation is related with a higher monocyte infiltration into the adipose tissue, leading to inflammatory and oxidative stress impairments. Antioxidant-based treatments constitute an interesting approach to counteract these phenomena.

Methods: Cocultures of differentiated 3T3-L1 adipocytes and RAW264 macrophages were incubated with $100 \mathrm{nM}$ insulin and 200 $\mathrm{M}$ vitamin C (VC) for $24 \mathrm{~h}$. Different inflammatory and oxidative stress markers, and the expression of some important adipokines, were assessed.

Results: Intra and extracellular ROS levels were induced in coculture. In contrast, VC incubation inhibited intracellular ROS concentration in both cultures, but more drastically in cocultures. Nitric oxide secretion was increased in the coculture system, and an interaction between insulin and $\mathrm{VC}$ was detected on the coculture system. MCP-1 mRNA expression was augmented by the coculture, and reduced by the insulin incubation in both cultures. VC incubation inhibits MCP-1 expression only in the control culture. Apelin gene expression was induced by the coculture and by the insulin treatment, and interestingly, an interaction between $\mathrm{VC}$ and the culture system was observed. Finally, leptin mRNA expression was diminished in coculture, and presented no effects by VC.

Conclusions: In summary, VC modulates inflammatory changes, adipokine synthesis and gene expression regulation in the interaction between adipocytes and macrophages.

\section{P093 \\ Pro12Ala Polymorphism of the PPARg2 Gene Increases Obesity Risk in an Elderly Population of the Sun Study}

\author{
Cecilia Galbete" ${ }^{1}$ Jon Toledo², Adriana Moleres" \\ Rafael Contreras', J. Alfredo Martínez', \\ Miguel Ángel Martínez-González³, Francisco Guillen- \\ Grima ${ }^{3}$, Amelia Marti ${ }^{1}$ \\ ${ }^{1}$ University of Navarra-Pamplona, Spain, ${ }^{2}$ Neurology \\ Service Donostia Hospital-San Sebastian, ${ }^{3}$ Department of \\ Preventive Medicine and Public Health, University of \\ Navarra, Pamplona, Spain
}

Introduction: Allelic variations in the peroxisome proliferatoractivated receptor gamma 2 gene (PPARg2) alter the risk for adiposity in adults, with evidence of interaction between dietary and lifestyle. The aim of this study was to analyze the relationship between the Pro12Ala polymorphism of the PPARg2 gene and obesity in an elderly population of the SUN (Seguimiento Universidad de Navarra) prospective cohort study.
Subjects and Methods: Subjects $(\mathrm{n}=941)$ aged 57 to $91(67 \pm 6$ years) were recruited from the SUN prospective cohort study $(70 \%$ male) being the obesity frequency $10 \%$ (19\% female). Lifestyle and dietary data were compiled by validated self-reported questionnaires. Individuals were genotyped for the rs1801282 SNP of the PPARg2 gene by RT-PCR followed by allele discrimination. Statistical analysis was performed by no conditional logistic regression adjusted for sex, age, physical activity, total energy intake and CHO intake.

Results: The allele frequency was 0.16 for Pro12Ala subjects and 0.01 for Ala12Ala. Elderly population having the Ala allele had higher risk of obesity (OR 1.66, 95\% CI 1.00-2.73, $\mathrm{p}=0.048$ ). Our results suggested that women have higher obesity predisposition linked to the 12Ala polymorphism (OR 3.93, 95\% CI, 1.43-10.20, $\mathrm{p}=0.008$ ). Subjects carrying the mutation with a high $\mathrm{CHO}$ intake presented a higher risk for obesity (OR 2.60, 95\% CI, 1.26-5.34, $\mathrm{p}=0.009$ ). Those persons carrying the mutation with lower physical activity (METs-h/week) had significantly higher risk of obesity (OR $2.18,95 \%$ CI 1.15-4.14, $\mathrm{p}=0.018$ ) than those with higher MET.

Conclusion: In this elderly population from the SUN study the Pro12Ala polymorphism of PPARg2 seems to be associated with obesity. Furthermore, the risk of obesity in subjects with the mutation is higher in women than in men and in subjects with a high CHO intake, while decreases in subjects with high physical activity.

\section{P094 \\ Genomic Analysis of Tenomodulin Gene in Obese Children}

\author{
Concepción M. Aguilera', Josune Olza', \\ Azahara I. Rupérez', Mercedes Gil-Campos², \\ Rosaura Leis ${ }^{3}$, Ramón Tojo ${ }^{3}$, Ramón Cañete ${ }^{2}$, Ángel Gil \\ ${ }^{1}$ Institute of Nutrition and Food Technology, Univer- \\ Granada, Spain, ${ }^{2}$ Reina Sofia University Hospital-Córdoba, \\ Spain, ${ }^{3}$ Clinic University Hospital of Santiago-Santiago de \\ Compostela, Spain
}

Introduction: Tenomodulin (TNMD) (locus Xq22) is expressed in both adipocyte and stromal vascular fraction of adipose tissue (AT). Weight loss decreases the expression of the TNMD mRNA in the AT, and the expression level is strongly correlated with body mass index in adults. Modest association between TNMD sequence variation and different obesity-related phenotypes has been described. Tenomodulin inhibits angiogenesis, but its specific function in AT is still unknown. This study aimed to determine whether TNMD variants and AT gene expression were associated with obesity in Spanish children.

Methods: Two single nucleotide polymorphisms (SNPs) in TNMD gene (rs932437 and rs11798018) were genotyped in 555 children (246 obese and 309 normal-weights). The SNPs were searched in the HapMap and NCBI databases among those with a minor allele frequency higher than 0.05 and $\mathrm{r} 2>0.8$ in the tagSNPs. The analysis was performed on the Golden Gate assay (Illumina). The AT gene expression analysis were carried out in 20 children (10 obese and 10 normal-weight) using the U133 Plus 2.0 Affymetrix array.

Results: A case-control association analysis revealed that both SNPs were associated with obesity in girls following an over dominant model; rs 11798018 odds ratio (OR) adjusted by age of 1.85 [95\% confidential interval $(\mathrm{CI})=1.04-3.29$ and $\mathrm{P}=0.032]$ and $\mathrm{rs} 932437$ 
$\mathrm{OR}=1.82(\mathrm{CI}=1.05-3.15 ; \mathrm{P}=0.031)$. Obesity-associated phenotypes, which include plasma fasting insulin, total cholesterol, HDL and LDL cholesterol, ApoA and ApoB were associated with those TNMD variants. Among all genes analyzed in the expression array, the TNMD showed one of the highest over expression (Fold change, $\mathrm{FC}=7.2$; $\mathrm{p}=0.0028)$ in obese AT. This result was validated by $\mathrm{RTPCR}(\mathrm{FC}=5.55$; $\mathrm{P}=0.028$ ). Inmunocytochemistry analysis confirmed the protein expression in adipocytes membranes.

Conclusion: The alteration in the sequence and AT expression of TNMD gene may play a role in the etiology of childhood obesity.

\section{P095}

\section{Effects of Lipoic Acid on Adipose-specific Phospholipase A2 (AdPLA)}

Marta Fernández-Galilea', Patricia Pérez-Matute², Pedro L. Prieto-Hontoria', J. Alfredo Martínez', María J. Moreno-Aliaga ${ }^{1}$

${ }^{1}$ University of Navarra-Pamplona, Spain, ${ }^{2} \mathrm{HIV}$ and Associated Metabolic Alterations Unit. Infectious Disease Area. Center for Biomedical Research of La Rioja (CIBIR)Logroño, Spain

Introduction: Previous studies have found that Lipoic acid (LA), an antioxidant molecule with beneficial effects on obesity, has lipolytic properties. Recently, a new phospholipase named AdPLA has been discovered and related to the inhibition of the lipolytic process. However, AdPLA regulation still remains unclear. The aim of the present study was to investigate the mechanisms involved in the lipolytic actions of LA, focusing on its potential ability to down regulate AdPLA.

Methods: Fully differentiated 3T3-L1 adipocytes were treated for 24 hours with different LA concentrations $(1-500 \mu \mathrm{M})$ in presence or absence of the JNK inhibitor SP600125, the MAPK inhibitor PD98069, the AKT inhibitor LY294002, the AMPK inhibitor Compound C, the PKA inhibitor H89, the PKG inhibitor KT5823, and the AMPK activator AICAR. Lipolysis was estimated as the amount of glycerol released to the media. AdPLA protein levels were assessed by western blot and AdPLA mRNA levels were determined by real-time PCR.

Results: LA stimulates lipolysis in a dose and time-dependent manner. Moreover, treatment with LA $(250 \mu \mathrm{M})$ inhibited both AdPLA gene expression and protein levels $(p<0.05)$. Pre-treatment with the specific inhibitors was not able to reverse the LA-induced down-regulation of ADPLA. However, the presence of the AKT inhibitor LY294002 and the PKA inhibitor H89 decreased basal AdPLA gene expression $(p<0.01$ and $p<0.05$ respectively), suggesting the involvement of these pathways in the regulation of AdPLA. Furthermore, in the presence of these inhibitors, no differences in ADPLA mRNA were observed between vehicle and LA-treated cells.

Conclusion: Our data suggest that the inhibition of the phospholipase AdPLA is likely to be involved in the lipolytic action of LA in 3T3-L1 cells.

\section{P096 \\ The TCF7L2, PGC-1 Genetic Variant in Spanish Obese Children - A Preliminary Study}

Carina Zabena', Gloria Bueno², María Teresa Martínez Larrad', Olga Bueno', José Antonio Mateos², S. Pinillos², Luis Morenoํ. Manuel Bueno ${ }^{2}$, Manuel Serrano Ríos ${ }^{1}$

${ }^{1} \mathrm{CIBER}$ de Diabetes y Enfermedades Metabólicas (CIBERDEM), Hospital Clínico San Carlos-Madrid, Spain,

${ }^{2}$ Hospital Clínico Universitario-Zaragoza

The aim of the study is to investigate the potential associations between the rs12255372 and rs7903146 of TCF7L2, Gly482Ser PGC-1 and hyperglycaemia, insulin resistance (IR), and related abnormalities in 174 obese children. We studied 174 obese children (54.7\% females); ages: 5-15 years, who attended the Pediatric Departments of two-hospitals (San Juan de Dios-Barcelona/Hospital Clínico Universitario, Zaragoza, Spain).

Methods: Body Mass Index (BMI), waist circumference, SBP, DBP. Fasting glucose/Lipid profile/insulin/proinsulin/leptin/adiponectin (RIA) Retinol Binding Protein (RBP4), adipocyte fatty acid binding protein (A-FABP): ELISA. Hyperglycaemia was defined as fasting glucose $100 \mathrm{mg} / \mathrm{dl}$. IR by HOMAIR. Genotyping: rs12255372 and rs7903146 of TCF7L2 gene: carried out using TaqMan SNP Genotyping Assays-ABI 7900 TH Fast PCR-Real-Time; Gly482Ser of PGC-1 gene: PCR-RFLP.

Results: Minor allele frequencies for: rs12255372 and rs7903146 of TCF7L2 gene ( 0.28 and 0.24 respectively) and Gly482Ser of PGC-1 (0.43). The female group showed higher levels of proinsulin, leptin and HOMAIR than male group. Prevalence of hyperglycaemia: 13.0\%. A-FABP levels correlations: BMI (r: 0.334; p>0.001), Fasting Glucose (r: 0.201 p: 0.023 ), even after adjustment age and sex. Female group carrying the Ser482 allele presented higher levels of A-FABP ( $\mathrm{p}=0.038)$ than those with the Gly482Gly genotype. This difference was significant even after adjustment by age, BMI (5.916 SE: 0.1997 p: 0.004). Also, subjects carrying the T allele of rs 7903146 of TCF7L2 presented higher levels of BMI, even after adjustment of age, sex (1.482 SE: 0.598 p: 0.014) than those with GG genotype. However, it was not found any association between rs 12255372 and anthropometric/biochemical features. Neither, any associations between three polymorphism and IR.

Conclusions: Our results suggest that: 1-A-FABP presented positive correlations with BMI and Fasting glucose levels regardless of age/sex; 2-The Ser482 allele of PGC-1 is likely associated with higher levels of A-FABP; 3-the rs7903146 T allele of TCF7L2 gene may be associated with increased BMI independently of age/sex. 
P097

\section{Growth Hormone Secretagogue Receptor (GHSR) Gene Polymorphisms Association with Food Intake and Metabolic Features in Obese Children and Adolescents}

Thais Arthur, Sophie Deram, Isabel Guazzelli, Eliana Frazzatto, Sandra Villares

University of Sao Paulo, Brazil

Introduction: Ghrelin, a hormone-releasing peptide, enhances appetite and increases food intake in healthy men. The endogenous ligand ghrelin is the growth hormone secretagogue receptor (GHSR), and effects of ghrelin are mediated via the GHSR. Ghrelin and the GHSR are believed to have important roles in energy homeostasis and metabolic parameters. Common polymorphisms in GHSR have been associated with obesity. The GHSR gene 477G/A (rs572169) polymorphism is a good candidate for susceptibility to obesity, and subjects carrying the minor A allele had a higher dietary restraint and more disinhibition than subjects homozygous for the $\mathrm{G}$ allele.

Objective: Determine possible associations between the $477 \mathrm{G} / \mathrm{A}$ (rs572169) polymorphism of GHSR with energy intake, macronutrient intakes and metabolic features in obese children and adolescents.

Methods: A total of 347 obese children and adolescents $(37.2 \%$ boys; aged $10.6 \pm 1,4$ years, BMI $30.5 \pm 4.7 \mathrm{~kg} / \mathrm{m}^{2}$, ZBMI $2.3 \pm 0.3$, $46.3 \%$ pubertal) were genotyped for GHSR by PCR. Metabolic measures were obtained and. energy and macronutrient intakes were assessed by using 3-day food records. The statistical analysis was performed with Independent-samples T test by SPSS software.

Results: The population was in Hardy-Weinberg equilibrium (GG: $67.4 \%$, GA: $29.7 \%$, AA: 2,9\%, p=0.74). GG children had higher energy intake that children carrying the $A$ allele (GA+AA) $(2127.7 \pm 641.2 \mathrm{Kcal}$ vs. $1815.3 \pm 562.0 \mathrm{Kcal}, \mathrm{p}=0.006)$ and carbohydrate intake (261.0 $\pm 86.9 \mathrm{~g}$ vs. $218.0 \pm 71.5 \mathrm{~g}, \mathrm{p}=0.005)$. Moreover, children carrying the A allele, when compared to GG, had lower total cholesterol ( $160.7 \pm 31.2 \mathrm{mg} / \mathrm{dL}$ vs. $152.5 \pm 27.4 \mathrm{mg} / \mathrm{dL}, \mathrm{p}=0.02)$, lower LDLc $(98.8 \pm 27.1 \mathrm{mg} / \mathrm{dL}$ vs. $89.7 \pm 25.7 \mathrm{mg} / \mathrm{dL}, \mathrm{p}=0.003)$ and higher HDLc $(41.1 \pm 8.8 \mathrm{mg} / \mathrm{dL}$ vs. $43.5 \pm 10.1, \mathrm{p}=0.03)$.

Conclusions: The results support the notion that the studied 477 G/A (rs572169) polymorphism of GHSR may be involved in obesity, food intake and metabolic alterations in obese children and adolescents.

\section{P098}

Anti-inflammatory Effects of $\omega-3$ polyunsaturated Fatty Acid and Hydroxytyrosol in Murine 3T3-L1 Adipocytes - A Protective Mechanism of Mediterranean Diets in Obesity

\author{
Egeria Scoditti ${ }^{1}$, Marika Massaro', \\ María Annunziata Carluccio ${ }^{1}$, Amalia Gastaldelli', \\ Carlo Storelli², Raffaele De Caterina ${ }^{3}$ \\ ${ }^{1}$ C.N.R. Institute of Clinical Physiology, Pisa and Lecce, \\ Italy, ${ }^{2}$ Department of Biological and Environmental \\ Science and Technolgy (Disteba), University of Salento- \\ Lecce, Italy, ${ }^{3}$ "G. d'Annunzio" University-Chieti, Italy
}

Background and Aim: Obesity is associated with increased risk of cardio-metabolic disorders. Excess adiposity results in enhanced oxidative stress and dysregulation of adipocytokine production, and is accompanied by macrophage infiltration in the adipose tissue, contributing to local and systemic inflammation. Mediterranean diets (MD) are associated with protection against obesity and type 2 diabetes through incompletely elucidated mechanisms. We investigated the effects of the MD components $\omega-3$ fatty acid docosahexaenoic acid (DHA) and the olive oil phenolic antioxidant hydroxytyrosol (HT) on adipogenesis and inflammation in murine 3T3-L1 adipocytes.

Materials and Methods: Differentiating 3T3-L1 adipocytes were incubated for 10 days with either $25-100 \mu \mathrm{mol} / \mathrm{L}$ DHA or $1-50$ $\mu \mathrm{mol} / \mathrm{L}$ HT. Lipid accumulation and PPARgamma expression were then determined by Oil Red-O staining and Western analysis, respectively. Differentiated adipocytes were treated with DHA for $48 \mathrm{~h}$ or HT for $1 \mathrm{~h}$ before $10 \mathrm{ng} / \mathrm{mL}$ TNF-stimulation for $30 \mathrm{~h}$. Levels of monocyte chemo attractant protein(MCP)-1 and adiponectin in the culture medium, and the surface expression of intercellular adhesion molecule(ICAM)-1 were measured by enzyme immune assays (EIA).

Results and Conclusions: In the absence of any toxicity, DHA and HT treatments decreased lipid accumulation and PPARgamma expression in differentiating adipocytes. Under pro-inflammatory conditions, as induced by exposure to TNF-alpha, mature adipocytes increased the release of MCP-1, the surface expression of ICAM-1, and reduced the release of adiponectin. Pretreatment with DHA or HT inhibited TNF-alpha-induced MCP-1 release and ICAM-1 expression, and reverted TNF-alpha-mediated inhibition of adiponectin. Our data suggest that specific MD compounds, including DHA and HT, exert specific anti-adipogenic actions in vitro and improve the inflammatory derangement of adipocytes restoring adiponectin secretion and attenuating MCP-1 and ICAM-1 expression, both involved in macrophage recruitment into the adipose tissue. 
P099

\section{Copy Number Variation of the Salivary Amylase Gene (AMY1) in Chilean Obese Children: Association with Insulin Resistance and Eating Behaviour Scores}

José L. Santos ${ }^{1}$, Ester Saus ${ }^{2,3}$, Mónica Gratacos ${ }^{2,3}$, Alberto Maiz', Rodrigo Cataldo', Xavier Estivill2,3,4

${ }^{1}$ Department of Nutrition, Diabetes and Metabolism, Pontificia Universidad Católica de Chile-Santiago, Chile, ${ }^{2}$ Genes and Diseases Program, Centre for Genomic Regulation-Barcelona, Spain, ${ }^{3}$ Ciber en Epidemiología y Salud Pública, CIBERESP, ${ }^{4}$ Pompeu Fabra UniversityBarcelona, Spain

Introduction: Salivary alpha-amylase catalyses the hydrolysis of dietary starch in the oral cavity. The human salivary amylase gene (AMY1) shows extensive Copy Number Variation (CNV) which is directly proportional to its protein expression in saliva. It has been suggested that inhibitors of alpha-amylase reduce food intake and increases glycaemia.

Aim: To estimate the association between copy-number variations of the salivary alpha-amylase gene with eating behaviour scores, basal glycaemia and insulin resistance in Chilean obese children.

Methods: Quantitative Polymerase Chain Reaction was used to estimate CNV of AMY1 gene in 130 Chilean children aged 8-12 years. We amplified human AMY1 and TP53 (control gene) in a PCR mixture containing SYBR Green I as DNA binding dye. Chimpanzee DNA was used as a control for a known number of AMY1 copies. Eating behaviour scores were calculated using the Child Eating Behaviour Questionnaire (CEBQ) adapted for Chile. CEBQ subscales showed high internal consistency (Cronbach's alpha $>0.8$ ) and an adequate replication of the original structure of eight factors, as shown by the multivariate factorial analysis. HOMA index was calculated from fasting glucose and insulin levels in serum, measured through a colorimetric assay and by radioimmunoassay respectively.

Results: The estimate of diploid CNV of AMY1 gene in Chilean subjects ranged from 2 to 20, with a mean and standard deviation of 6.5 ,b 2.9. No significant associations were detected between AMY1 $\mathrm{CNV}$ and glycaemia or HOMA index after adjustment for age and sex. Likewise, no significant results were obtained for the association between AMY1 CNV and eating behaviour scores.

Conclusion: We found a wide range of copy-number variation in the AMY1 gene in the Chilean population. There is insufficient evidence to support a major effect of CNV of AMY1 gene on basal glycaemia, HOMA index and eating.
P100

\section{Lipidomic Profile of Adipose Tissue in Non Obese (BMI $<30 \mathrm{Kg} / \mathrm{m}^{2}$ ), Obese (BMI $30-39.99 \mathrm{Kg} / \mathrm{m}^{2}$ ) and Morbidly Obese (BMI $\geq \mathbf{4 0} \mathbf{K g} / \mathrm{m}^{2}$ ) Patients. (Preliminary Study)}

Carina Zabena, Alma Astudillo, María Teresa Martínez Larrad, Luis Gil de Gómez, Noelia Rodríguez Pérez, Arturo Corbaton Anchuelo, Jesús Álvarez Fernández Represa, Jesús Balsinde, Manuel Serrano Ríos

CIBER de Diabetes y Enfermedades Metabólicas Asociadas-Madrid, Spain

Aims: The purpose of our study was to identify the lipidomic profile of adipose tissue samples from patients according to degree of BMI (23.77-67.12 kg/m²) and its correlation with selected metabolic parameters.

Subjects: 49 unrelated subjects (28 females, 57.14\%) patients submitted to bariatric or selective (non obese patients) surgery. Study groups: Non obese (NO) BMI $<30 \mathrm{~kg} / \mathrm{m}^{2}(\mathrm{n}=6,33.3 \%)$, Obese (O) BMI 30-39.99 kg/m² (n=10, 70.0\%) and Morbidly Obese (MO): BMI $\geq 40 \mathrm{~kg} / \mathrm{m}^{2}(\mathrm{n}=33,57.6 \%)$.

Methods: Weight $(\mathrm{kg})$, height $(\mathrm{m}), \mathrm{SBP}$ and DBP $(\mathrm{mmHg})$ were measured. BMI $\left(\mathrm{kg} / \mathrm{m}^{2}\right)$ was estimated. Fasting glucose $(\mathrm{FG}) /$ lipid profile/Insulin levels (RIA). Insulin resistance (IR) by HOMAIR, Average values for Spanish population HOMAIR $\geq 3$. 8 . Adipose tissue (AT) (SAT: subcutaneous, $n=49$ and OAT: omental, $n=41$ ) samples were obtained during surgery. Lipidomics analyses by gas chromatography coupled to mass spectrometry.

Results: Prevalence of impaired FG: NO: 16.7\%, O:50.0\% MO: $66.7 \%, p=0.132$; and IR: NO: $16.7 \%, \mathrm{O}: 60.0 \% \mathrm{MO}: 84.8 \%, \mathrm{p}=0.047$. Lipidomics study: Up to 17 fatty acids were detected in triacilglycerol (TAG) samples. In SAT in MO-patients the lipidomic profile revealed higher expression of pentadecanoic-acid (TAG15:0)/palmitic-acid (TAG16:0)/stearic-acid (TAG18:0)/11-14-eico sadienoic-acid (TAG20:2n-6), 8,11,14 -eicosatrienoic-acid (TAG20:3n-6)/arachidonic-acid (T AG20:4n-6), and total TAG as compared to O-patients. In MO-patients there were increased levels of palmitoleic-acid (TAG16:1n-7) but decreased levels of lauric-acid (TAG12:0) as compared to NO-patients. In OAT MO patients presented higher levels of TAG and decreased levels of TAG18:0 than O-patients. Ratio SAT/ OAT:in MO-patients revealed higher oleic-acid (TAG18:1n-9c) as compared to O-patients.

Conclusions: Our results suggest that: a-different SAT and OAT lipidomic profiles were apparent in $\mathrm{O}$ and $\mathrm{MO}$ patients, b-Increased levels of polyunsaturated species (TAG20:2n-6, TAG20:3n-6 and TAG20:4n-6) in SAT TAG in MO as compared to O are strongly associated with higher level of IR. 


\section{P101 \\ Polymorphism rs 1137101 in LEPR is Not Associated with Binge Eating Behavior in Obese Children}

\author{
Thais Arthur, María Edna Melo, Aritania Santos, \\ Clarissa Fujiwara, Heidi Reinhardt, Alfredo Halpern, \\ Marcio Mancini \\ University of Sao Paulo, Brazil
}

Introduction: Leptin is an important regulator of adipose tissue mass and has been associated with obesity and metabolic disturbances. Leptin exerts its effects through interaction with leptin receptor (LEPR). LEPR plays a crucial role in regulation of body weight. Several common polymorphisms have been described in the human LEPR gene including rs 1137107 (CAG $>$ CGG - Q223R). The association of this polymorphism with obesity or related metabolic phenotypes has been controversial. Binge eating may be originated in neural and hormonal defects in appetite and satiety control. Although the prevalence of binge eating disorder (BED) as defined in DSM-IV in the normal adult population ranges from $2 \%$ to $5 \%$, rates in cohorts of obese adults seeking treatment for obesity rise up to $30 \%$. No data related to rs 1137107 influence on BE is available.

Objective: To verify associations between rs1137101 in LEPR and binge eating behavior and metabolic parameters in obese children.

Methods: 112 obese children (47.3\% boys; $12.5 \pm 2.7$ years, ZBMI 2.37 \pm 0.34 , 63 pubertal) were genotyped for rs 1137107 by restriction fragment length polymorphism. The binge eating score was classified according to the Binge Eating Scale (BES). Children were classified as binge eaters if they scored $>18$. The statistical analysis was performed with qui-square and one-way ANOVA tests by SPSS software.

Results: The population was in Hardy-Weinberg equilibrium (AA: $32.5 \%$, AG: $46.5 \%$, GG: $21.0 \%$, p=0.54). We found $56(52.3 \%$ ) binge eaters without association with $\mathrm{rs} 1137107(\mathrm{p}=0.396)$. Average values of BES according to the presence of rs1137107 were not different (AA: 18.35, AG: 16.96 and GG: 18.91; $\mathrm{p}=0.562$ ). Children carrying the allele $\mathrm{G}$ did not have more metabolic disorders, without variation in triglycerides level $(p=0.48)$, glicemia level $(p=0.24)$ and HDL cholesterol level $(\mathrm{p}=0.24)$.

Conclusions: Our results suggest that the polymorphism rs 1137107 in LEPR is not involved in binge eating behavior and metabolic parameters in obese children.

\section{P102 \\ FTO rs9939609 Influences Obesity and Physical Activity in a Mediterranean Population (Garaulet Study)}

Carmen Sánchez Moreno", José María Ordovás ${ }^{2,3}$, Eva Morales-Falo' , Juan José Alburquerque-Béjar ${ }^{1}$, Mari Paz Carrasco-Benso', Jesús López-Mínguez', Marta Garaulet ${ }^{1}, 2$

${ }^{1}$ University of Murcia, Spain, ${ }^{2} J$ Jean Mayer US Department of Agriculture Human Nutrition, Research Center on Aging, Tufts University School of Medicine, ${ }^{3}$ The Department of Epidemiology and Population Genetics, Centro Nacional Investigación Cardiovasculares (CNIC), Spain

Introduction: FTO gene has been previously related to obesity. However, data are still conflicting and replications are needed in different populations, particularly in people following a Mediterranean type of diet.

Methods and Materials: We recruited 1500 subjects from a Mediterranean population (BMI: $25-40 \mathrm{~kg} / \mathrm{m}^{2}$ ) of $20-65 \mathrm{y}$. who attended outpatient obesity clinics in Spain. Total energy intake and macronutrient composition were analyzed with the nutritional evaluation software program Grunumur on the basis of Spanish food composition tables.

Results: We found significant associations between the FTO rs9939609 variants and obesity traits as body fat percentage $(\mathrm{P}=$ $0.020)$ and BMI $(P=0.020)$, with higher obesity degree in minor allele (A) carriers. Furthermore, FTO rs9939609 associated with physical activity. In general, minor allele carriers reported lower rates of exercise than non-carriers. We found a new gene-diet interaction between the FTO gene and Saturated Fat intake (SF) for obesity $(\mathrm{P}=$ $0.0016)$ with a significantly higher BMI in (A) carriers than in noncarriers $(35.97 \mathrm{~g} \pm 0.45$ vs $33.83 \mathrm{~g} \pm 0.62)(\mathrm{Mean} \pm \mathrm{SEM})$ among the high $\mathrm{SF}$ intake group of patients. No significant differences were found in obesity degree among FTO variants in patients with low intakes of SF.

Conclusions: We have replicated the previous association between FTO rs9939609 and obesity, and we have reported a new association with physical activity in patients following a Mediterranean Diet. The minor allele appears to contribute to the accumulation of body fat in people who also displayed lower rates of exercise. Interestingly we have detected a gene-diet interaction between FTO and saturated fat intake for obesity. 
Other Acts

\section{P103 \\ Folic Acid Supplementation of the Maternal Diet During Gestation Decreases Expression of PPAR and LXR Genes in the Progeny}

\author{
Agata Chmurzynska', Monika Stachowiak ${ }^{2}$ \\ ${ }^{1}$ Poznan University of Life Sciences, Poland, ${ }^{2}$ Department \\ of Genetics and Animal Breeding- Poznan, Poland
}

Composition of the maternal diet during gestation has been shown to determine growth, body composition, and lipid metabolism of the progeny in postnatal life. Regulation of lipid metabolism is achieved by the action of several nuclear receptors including peroxisome proliferator-activated receptors (PPARs) and liver X receptors (LXRs). The aim of this study was to determine the effect of folic acid and protein content in the maternal diet during gestation on hepatic and adipose tissue expression of PPAR and LXR genes in the progeny. Pregnant female rats were fed one of four experimental diets: a control diet (AIN-93) containing adequate amounts of protein and folic acid, a protein-restricted diet without folic acid supplementation, a protein-restricted diet with folic acid supplementation, and a folic acid supplemented diet with a normal level of protein. Hepatic and adipose tissue expression of PPAR and LXR genes was analyzed. Folic acid supplementation of the dams' diet reduced the transcription level of PPAR in the liver and adipose tissue and also decreased LXR expression in the liver. These results indicate that folic acid content in the maternal diet affects PPAR and LXR in the offspring, and may therefore contribute to the regulation of lipid metabolism.

\section{P104}

\section{Adequate Protein Content and Folic Acid Supplementation of the Maternal Diet During Gestation Downregulates Enzymes Involved in the Methionine Cycle in the Progeny}

\section{Agata Chmurzynska, Anna Malinowska \\ Poznan University of Life Sciences-Helsinki, Finland}

The metabolism of methionine is dependent on the availability of several nutrients, including folates and vitamins B6 and B12, and has been shown to play an important role in the fetal programming phenomenon. The study was intended to analyze the influence of maternal diet during pregnancy on the hepatic expression of cystathionine beta synthase (CBS), betaine-homocysteine methyltransferase (BHMT), and phosphatidylethanolamine N-methyltransferase (PEMT) genes in the progeny. Pregnant female rats were fed one of four experimental diets: a control diet (AIN-93), a protein-restricted diet without folic acid supplementation, a protein-restricted diet with folic acid supplementation, and a folic acid supplemented diet with a normal level of protein. After weaning, the offspring of each maternal dietary group was fed a balanced or a high-fat diet. There was a significant interaction effect of protein and folic acid content in the maternal diet on CBS, BHMT, and PEMT expression, which was reduced in the progeny of dams, fed the folic acid supplemented diet with an adequate level of protein. Moreover, the high-fat feeding modified the gene transcription level. Therefore the interplay between prenatal and postnatal dietary factors may determine the effectiveness of the methionine cycle.

\section{P105 \\ Postprandial Antioxidant Effect of the Mediterranean Diet Supplemented with Coenzyme $\mathbf{Q 1 0}$ in Elderly Men and Women \\ Elena María Yubero Serrano ${ }^{1}$, Nieves Delgado-Casado", Pablo Pérez-Martinez ${ }^{1}$, Inmaculada Tasset-Cuevas ${ }^{2}$, Francisco Fuentes', Javier Delgado-Lista1, Isaac Tunez², José Manuel Villalba ${ }^{3}$, Francisco Pérez-Jiménez', José López-Miranda ${ }^{1}$ \\ ${ }^{1} \mathrm{IMIBIC/Reina} \mathrm{Sofia} \mathrm{University} \mathrm{Hospital/University-}$ Cordoba, Spain, ${ }^{2}$ Dept. of Biochemistry and Molecular Biology IMIBIC, ${ }^{3}$ Dept. of Cell Biology, Physiology and Immunology, Spain}

Introduction: Aging is the result of the accumulation of cellular and tissue damage caused by the endogenous generation of reactive oxygen species (ROS). Coenzyme Q10 (CoQ) acts as a powerful antioxidant that reduces oxidative stress.

Methods and Materials: We have investigated whether the type of dietary fat and the supplementation with CoQ affect oxidative stress in an elderly Caucasian population. In this randomized crossover study, 10 men and 10 women were assigned to receive three isocaloric diets for periods of $4 \mathrm{wk}$ each. 1. Mediterranean diet supplemented with CoQ (Med $+\mathrm{CoQ}$ diet). 2. Mediterranean diet (Med diet). 3. SFA-rich diet (SFA diet). After each dietary period plasma levels of CoQ, alpha-tocopherol, ascorbic acid, beta-carotene, lipid peroxides (LPO), oxidized LDL (oxLDL), protein carbonyl (PC), total nitrite, nitrotyrosine and catalase, superoxide dismutase (SOD) and glutathione peroxidase activities in plasma were determined.

Results: When the participants consumed the Med diet, plasma concentration of beta-carotene and total nitrite were greater and oxLDL and SOD activity were lower than when they consumed the SFA diet. Supplementation of Med diet with CoQ induced higher levels of beta-carotene, alpha-tocopherol, ascorbic acid, total nitrite and lower levels of oxLDL, SOD and catalase activity compared with the Med and the SFA diets, and lower concentrations of nitrotyrosine, LPO and PC compared with the SFA diet.

Conclusions: In summary, Med diet reduces processes of cellular oxidation and increases the action of antioxidant system in elderly persons and the administration of CoQ further improves this situation. These data may provide a starting point for the prevention of the oxidation generated during the aging process. 


\section{P106 \\ Mediterranean Diet Supplemented with Coenzyme $\mathbf{0 1 0}$ Modifies Postprandial Activation of p53 in Mononuclear Cells of Elderly Subjects in Response to DNA Damage}

Francisco Miguel Gutiérrez Mariscal, Pablo Pérez Martínez, Elena María Yubero Serrano, Antonio Camargo, Javier Delgado Lista, Nieves Delgado Casado, Francisco Fuentes, Francisco Pérez Jiménez, José López Miranda

IMIBIC/Hospital Universitario Reina Sofía, /Universidad de Córdoba and Ciber Fisiopatología Obesidad y Nutrición Instituto Salud Calos III, Spain

Dietary oxidative stress denotes a disturbance of the redox state resulting from excess oxidative load or from inadequate nutrient supply favouring prooxidant reactions. Coenzyme Q10 $(\mathrm{CoQ})$ is a powerful antioxidant that reduces oxidative stress and p53 mediates cellular response to DNA damage. We determine whether the quality of dietary fat alters oxidative DNA damage and whether supplementation with CoQ improves antioxidant capacity by modifying the activation / stabilization of p53 in elderly subjects.

Methods: In a crossover design twenty subjects were randomly assigned to received three isocaloric diets for four-weeks each: 1.Mediterranean diet (Med diet) 2.- Mediterranean diet supplemented with CoQ (Med+CoQ) 3.- SFA-rich diet (SFA diet). After $12 \mathrm{~h}$ fast, volunteers consumed a breakfast with a fat composition similar to that consumed in each of the diets. p53, phosphorylated p53 (Ser20), monoubiquitinated $\mathrm{p} 53$ and $\mathrm{p} 21$ proteins, both in cytoplasm and in nucleus of mononuclear cells were determined by Western Blot. p53, p21, p53R2 and Mdm2 mRNA expression were determined by qRTPCR. DNA damaged was measured as $8 \mathrm{OHdG}$ in serum by ELISA.

Results: SFA breakfast displayed higher postprandial concentrations of $8-\mathrm{OHdG}, \mathrm{mRNA}$ of $\mathrm{p} 53$ and monoubiquitinated $\mathrm{p} 53$ protein, and lower postprandial levels of mRNA Mdm2 compared with Med and Med + CoQ breakfasts $(\mathrm{p}<0.05)$. Moreover, Med + CoQ breakfast induced postprandial decreased of p53 cytoplasmatic, p-p53(Ser20) nuclear, and monoubiquitinated p53 protein nuclear and cytoplasmatic $(p<0.05)$. In conclusion, Mediterranean diet supplemented with CoQ increases the action of antioxidant systems and reduces processes of cellular oxidation. Our results suggest a starting point for the prevention of oxidative processes associated with aging.

\section{P107}

\section{Comparative Metabolic Fate of Alpha- linolenic Acid from Natural Oil and Structured Lipids in Rats}

\author{
Leslie Couedelo ${ }^{1}$, Carole Vaysse ${ }^{1}$, Nicole Combe1, \\ Emilie Vaique ${ }^{2}$, Alexandre Guy ${ }^{3}$, Thierry Durand ${ }^{3}$, \\ Sandra Pinet ${ }^{2}$, Maud Canse//4 \\ ${ }^{1}$ ITERG, Département Nutrition Santé-Talence Cedex, \\ France, ${ }^{2}$ Université Bordeaux 1, CNRS, ISM UMR-5255, \\ ENSCBP, ${ }^{3}$ Université Montpellier 1, Université Montpellier, \\ ${ }^{4}$ Université Bordeaux 1, CNRS, TREFLE UMR-8508, ENS, \\ France
}

Introduction: Human health benefits of the fatty acids of the n-3 series $(\omega 3)$ are well established for prevention of cognitive, cardiovascular, immune, and inflammatory diseases. Epidemiological studies in Western countries have demonstrated an insufficiency in the consumption of the alpha-linolenic acid (ALA, 18:3 n-3), precursor of the $\omega 3$ family. Thus, improving ALA bioavailability without increasing lipid intake is a real.

Material and Methods: Two parameters known to affect ALA metabolic fate were tested: 1) the physical form of the dietary lipids, i.e., flaxseed oil (triacylglycerols (TAG) containing $46 \mathrm{wt} \%$ of ALA) in emulsion versus in bulk phase; 2) the chemical form of the dietary lipids, i.e., pure symmetrical (ALA in sn-2 position) and unsymmetrical (ALA in sn-1 position) TAG were synthesized1. ALA in lymph was measured in thoracic lymph duct-cannulated rats after intragastric feeding of the different forms of TAG.

Results: Concerning the influence of the TAG physical form, emulsification led to a total higher ALA recovery in lymph $(+135 \%)$ compared with the same oil given in bulk phase. Moreover, maximal ALA absorption appeared earlier ( 2 hours before) and with a significant greater extent $(+55 \%, \mathrm{p}<0.05)$ in the emulsion group. Concerning the influence of the TAG chemical form, ALA intestinal absorption rate was comparable regardless of the structured TAG used. However, 24 hours after feeding, with symmetrical TAG, 30\% of ALA was recovered in the internal position of the lymphatic chylomicron TAG. In contrast, with unsymmetrical TAG, $90 \%$ of ALA was recovered in the external position of the lymphatic TAG. We conclude that intestinal recovery of ALA was improved by the flaxseed oil emulsification. On the other hand, the position of ALA of dietary TAG influences partially ALA distribution in chylomicrons TAG. 


\section{P108 \\ Knowledge, Attitudes and Practices of Macedonian and Croatian Consumers Towards the Personal Consumption of Fruits}

\author{
Igor Spiroski1, Ruzica Butigan², Ante Vuletic², \\ Vladimir Kendrovski ${ }^{1}$ \\ ${ }^{1}$ Institute of Public Health of the Republic of Macedonia- \\ Skopje, ${ }^{2}$ Faculty of Economics, University of Zagreb- \\ Croatia
}

Objective: The aim of this study was is to gain a qualitative understanding of different aspects of fruit consumption among consumers in Macedonia and Croatia.

Methods: 60 in-depth face to face facilitated, previously structured interviews were conducted in both countries, 30 per country. Consumers were recruited via screening interview from a recruitment questionnaire. They were randomly selected from all geographical regions in both countries.

Results: Taste was main reason for in general fruit consumption in both countries. Apple was mentioned as most prioritized fresh fruit for consumption in different settings, both in Macedonia and in Croatia. $80 \%$ and $75 \%$ consumers consume fruit at home in Macedonia and Croatia respectively. Level of sugar added in it was the main obstacle for processed fruit consumption in both countries. $37 \%$ of male respondents in Macedonia rarely or never consume dried fruit and $30 \%$ of males never do it in Croatia. Health protection and disease prevention is the most important factor for fresh fruit consumption in Croatian consumers while taste is the most important for Macedonian ones. Prize is most important obstacle for fresh fruit consumption in Croatia and seasonal availability is main obstacle in Macedonia. Majority of Macedonian (86\%) and Croatian (77\%) consumers eat fruit at least once a day with fresh apple being main fruit product. Still, for those consuming fruit more than twice a day, fruit juice is the main fruit source in both countries. $97 \%$ of Macedonian participants and $87 \%$ of Croatian think that it is recommended to eat at least 2 portions of fruit daily. The heart disease protection role of fruit is clear for $73 \%$ of Macedonian and $87 \%$ of Croatian respondents.

Conclusions: There is high consciousness of consumers in both countries about the importance of personal fruit consumption particularly towards health protection and disease prevention.

\section{P109 \\ Gene Expression Signatures in BALB3T3 Fibroblasts Exposed to lonic Versus Ultrafine Particulate Cobalt}

Silvia Perconti', Gitana María Aceto ${ }^{1,2}$, Francesco Napolitano ${ }^{3}$, Enrico Sabbioni ${ }^{1}$, Fabio Verginelli, ${ }^{1}$, Giancarlo Raiconi ${ }^{3}$, Roberto Tagliaferri3, Mario Di Gioacchin ${ }^{1}$, Renato Mariani-Costantini ${ }^{1,2}$

${ }^{1}$ Aging Research Center, G. d'Annunzio University Foundation-Chieti, Italy, ${ }^{2}$ Department of Oncology and Experimental Medicine, G. d'Annunzio University-Chieti, Italy, ${ }^{3}$ Department of Mathematics and Computer Science (DMI), University of Salerno, Italy

Introduction: Cobalt is a nutritionally essential trace metal, but at high doses is carcinogenic to animals and classified as potential human carcinogen by IARC. To explore relationships between biological effects and physical characteristics, we investigated the gene expression patterns of BALB3T3 A31-1-1 cells exposed to $1 \mathrm{M}$ concentrations of cobalt micro particles (Co-MP), cobalt nanoparticles (Co-NP), and cobalt ions (Co++).

Methods and Materials: Exposures were at semi-confluence for $72 \mathrm{hrs}$. Analyses were carried-out using 70-mer murine 13,443 oligonucleotide microarrays (Operon version 1.1). After normalization with MIDAS differentially expressed genes were detected by SAM analysis. Two-way hierarchical cluster analysis was performed using Cluster 3.0 and the dendrogram visualized by Java TreeView. The DAVID tool (http://david.abcc.ncifcrf.gov) was used to identify enriched Gene Ontology (GO) terms. Molecular interaction networks and canonical pathways analysis (Ingenuity software, http.//www. ingenuity.com) were performed on significant Gos. Key genes were confirmed by RT-PCR.

Results: SAM indicated that small percentages of genes were affected. Co-NP affected gene expression most, $\mathrm{Co}++$ least. The only gene affected by all treatments was RAB18, regulator of vesicular secretion. Unsupervised clustering identified two main clusters, a set of up-regulated genes, mostly with $\mathrm{Co}++$, and a set of down-regulated genes, mainly with Co-NP/Co-MP. Affected pathways and networks were: $\mathrm{Co}++$ : mitochondrial dysfunction; Co-NP: jak-STAT signaling, sphingosine-1-phosphate signaling, activation of interferon regulatory factor (IRF) family transcription factors, RIG1-like receptors, death receptors, and, in common with Co-MP (but only significant for Co-NP), aryl hydrocarbon signaling, apoptosis, polo-like kinases; Co-MP: nucleotide sugar metabolism, caveolar-mediated endocytosis signaling, nitric oxide signaling, starch/sucrose metabolism, and, in common with Co-NP (but only significant for Co-MP), G1/S checkpoint regulation, neuregulin signaling, androgen signaling, pyrimidine metabolism.

Conclusions: Cobalt in different physical forms appeared to cause divergent cellular responses that may have biological and toxicological relevance. Key genes in networks could represent candidate biomarkers of specific effects. 


\section{P110}

\section{Regulation of Cardiotrophin-1 Expression by Free Fatty Acids}

\begin{abstract}
Beatriz Marcos-Gómez', Eduardo Martínez-Anso², Idoia Martínez de la Piscina1, J. Alfredo Martínez", Jesús Prieto², Matilde Bustos², María J. Moreno-Aliaga ${ }^{1}$

${ }^{1}$ University of Navarra-Pamplona, Spain, ${ }^{2}$ Hepatology and Gene Therapy Area Center for Applied Medical Research (CIMA) Universtity of Navarra-Pamplona, Spain
\end{abstract}

Introduction: Cardiotrophin-1 (CT-1) is a member of the IL-6 cytokines family. It has been shown that adipocytes are able to produce CT-1, and that the circulating levels of CT-1 are up regulated in the metabolic syndrome. Moreover, it has been described that glucose up regulates CT-1 gene in adipocytes. The aim of this study was to investigate whether CT-1 levels are regulated by free fatty acids (FFA).

Material and Methods: The effects of FFA on CT- 1 mRNA levels and protein secretion were tested in fully differentiated 3T3-L1 adipocytes. Adipocytes were treated (1-16 h) with a mixture of FFA cocktail (100 and $200 \mathrm{M}$ in $0.1 \%$ FFA-free BSA) or vehicle. In order to study the in vivo effects of FFA on CT-1, overnight fasted mice were intravenously injected with either 2001 of a lipid infusion $(10 \%$ Lipofundin) or saline. Blood samples, white adipose tissue, liver and muscle were obtained from the animals at different times after injection (1, 3 and 5 h). CT-1 gene expression was determined by RT-PCR. Plasma CT-1 levels were measured by ELISA.

Results: Treatment of cultured adipocytes with a mixture of FFA induced a significant dose and time-dependent increase $(\mathrm{p}<0.05)$ in CT-1 gene expression and protein secretion. Moreover, CT-1 circulating levels and CT-1 gene expression in liver, adipose tissue and muscle were increased after the injection of a lipid infusion in parallel with the increase of plasma FFA in mice.

Conclusions: Our data demonstrate that $\mathrm{CT}-1$ is up regulated by FFA, suggesting that CT-1 is likely to be a nutritionally regulated cytokine. The potential mechanisms involved in the regulation of CT-1 by FFA have to be elucidated.

\section{P111}

Subcutaneous Adipose Tissue: Aproteomic Study in Morbidly Obese Patients According to the Range of their Body Mass Index (BMI)

\author{
Carina Zabena1, Petra Mateos Cáceres², \\ María Teresa Martínez Larrad', Javier Modrego", \\ Jesús Fernández Represa1, Antonio Torres García4, \\ Carlos Macaya², José Antonio López Farré2, \\ Manue/ Serrano Ríos ${ }^{1}$ \\ ${ }^{1}$ CIBER de Diabetes y Enfermedades Metabólicas \\ Asociadas (CIBERDEM) Laboratory of Diabetes and Lipids, \\ Hospital Clínico Universitario-Madrid, Spain, \\ ${ }^{2}$ Cardiovascular Research Unit Cardiovascular Institute \\ Hospital Clínico San Carlos-Madrid, Spain, ${ }^{3}$ CIBER de \\ Diabetes y Enfermedades Metabólicas Asociadas \\ (CIBERDEM), Surgery 1 Service, Hospital Clínico San \\ Carlos-Madrid, Spain, ${ }^{4}$ Surgery 3 Service Hospital Clínico \\ San Carlos-Madrid, Spain
}

The purpose of our study was to compare protein expression levels in subcutaneous adipose tissue (SAT) samples obtained from morbidly obese patients during bariatric surgery, within their range of Body Mass Index (BMI) [34.90-67.10 kg/m²] and several other metabolic parameters. We studied 19 unrelated morbidly obese patients (12 females, 63.2\%) who had undergone bariatric surgery. BMI was calculated $\left(\mathrm{kg} / \mathrm{m}^{2}\right)$. Systolic/diastolic blood pressures were measured. Fasting plasma glucose, lipid profile, proinsulin, insulin, leptin and adiponectin levels (RIA) were assayed. Insulin Resistance (IR) was estimated by $\mathrm{HOMA}_{\mathrm{IR}}$. SAT samples were obtained during bariatric surgery. Proteomic analysis was done using two-dimensional electrophoresis, mass spectrometry and western blot. The proteomic analysis of SAT revealed specific proteins and their isotypes, with higher levels of Peroxirredoxin 2 expression and one isotype of transferrin found in patients with BMI $\geq 50 \mathrm{~kg} / \mathrm{m}^{2}$ as compared to those with $\mathrm{BMI}<50 \mathrm{~kg} / \mathrm{m}^{2}$. The expression of four transferrin isotypes and Glutathione S-transferase (GST) protein were reduced in those patients with the higher BMI ( $\left.\geq 50 \mathrm{~kg} / \mathrm{m}^{2}\right)$. Our findings suggest that damage of SAT in morbidly obese patients with higher BMI $(\geq 50 \mathrm{~kg}$ / $\mathrm{m}^{2}$ ) is revealed by impaired selective protein expression levels correlated with their extremely high degree of adiposity. Although levels of stress response proteins were significantly altered, other proteins controlling specific metabolic functions (lipid, carbonic acid metabolism) remained preserved in this situation. 
P112

\section{Palmitic Acid Increases Nitric Oxide Production by Rat Skeletal Muscle Cells}

Rafael Herling Lambertucci ${ }^{1,2}$, Carol Góis Leandro ${ }^{3}$, Leonardo Dos Reis Silveira², Sandro Massao Hirabara ${ }^{1,2}$, Marco Aurélio Vinolo², Renato Tadeu Nachbar', Rui Curi², Tania Cristina Pithon-Curi ${ }^{1,2}$

${ }^{1}$ Cruzeiro Do Sul University, Post-Graduate Program-Säo Paulo, Brazil, ${ }^{2}$ University of São Paulo, Department of Physiology, ${ }^{3}$ Federal University of Pernambuco, Department of Nutrition, Brazil

Introduction: The ability to maintain skeletal muscle contraction is mainly dependent upon oxidation of fatty acids and glucose. Carbohydrate is predominantly used during high intensity exercise, whereas fatty acids are the predominant fuel during low to moderate intensity muscle contraction. Increased plasma concentrations of free fatty acids (FFA) lead to insulin resistance in skeletal muscle. In addition, high intracellular concentrations of FFA have impairing effects on mitochondrial function, including uncoupling of oxidative phosphorylation and decrease of endogenous antioxidant defenses. The effects of palmitic acid (PA $25 \mu \mathrm{M}$ ) on nitric oxide (NO) production by rat skeletal muscle cells and the possible mechanism involved were investigated.

Methods: Primary cultured rat skeletal muscle cells were treated with PA and NO production was assessed by nitrite measurement (Griess method) and by using 4,5 -diaminofluorescein diacetate (DAF-2-DA) assay. Nuclear factor-kappa B (NF-kappaB) activation was evaluated by electrophoretic mobility shift assay and iNOS protein content by western blotting.

Results: PA treatment increased nitric oxide production as measured by Griess method and DAF-2-DA assay. This effect was abolished by treatment with L-nitro-arginine (LNA) and L-nitro-arginine methyl esther (L-NAME) (NOS inhibitors). NF-kappaB activation and iNOS content was increased due to PA treatment. The participation of superoxide on nitric oxide production was investigated by incubation of cells with DAF-2-DA in the presence or absence of palmitic acid, a superoxide generator system (xanthine - xanthine oxidase - X-XO), a mixture of NOS inhibitors and SOD-PEG. Palmitic acid and $\mathrm{X}-\mathrm{XO}$ system increased $\mathrm{NO}$ production and this effect was abolished when cells were treated with NOS inhibitors and SODPEG.

Conclusions: the present study showed that PA stimulates NO production in cultured skeletal muscle cells through production of superoxide, nuclear factor-kappa B activation and increase of iNOS protein content.

\section{P113}

Sumo-Dependent Trascriptional Repression Mediated by Thyroid Hormone Receptor Isoforms Have Distinct Mechanisms

\author{
Marie Togashi ${ }^{1}$, Aleksandra Cvoro ${ }^{2}$, \\ Raysson Frota de Farias ${ }^{1}$, Paul Webb ${ }^{2}$ \\ ${ }^{1}$ Pharmaceutical Sciences Dept., University of Brasilia, \\ Brazil, 2Diabetes Research Center, the Methodist Hospital \\ Researh Institute-Houston, USA
}

SUMOylation, the covalent attachment of a $11 \mathrm{kDa}$ protein called SUMO-1 (Small Ubiquitin Like-Modifier-1) to proteins, participates in different functions in cell metabolism, including transcriptional regulation, nuclear transport, maintenance of genome integrity, and signal transduction. It's controlled by an enzyme pathway, analogous to ubiquitin pathway, that recognizes a consensus SUMOylation sequence, $\mathrm{KXD} / \mathrm{E}$, where is a hydrophobic residue and K the SUMO accepting lysine. Transpression activity induced by SUMOylation has been studied in nuclear receptors, as in recently reported anti-inflammatory responses mediated by PPAR, LXRs, and LRH-1. As thyroid hormone receptor (TR) plays an important role in many functions of the body, from homeostasis, development to cell metabolism, we aimed to identify SUMOylation motifs and study the effect of SUMO-1 in TR isoforms transcriptional activity. In gene reporter assays using HeLa cells, SUMO-1 over expression with TRalpha and TRbeta in the presence of increasing concentrations of hormone (T3) showed opposite effects in EC50 in IP6 response element: while in TRbeta, SUMO-1 increased EC50, in TRalpha EC50 was decreased. Increased repression in the absence of hormone was also observed for both TR isoforms. To address the location of SUMO binding site, we mutated all possible sites in both TRs and performed in vitro SUMOylation reactions in purified TRs ligand binding domains (LBDs). Mutation in only one site in TRbeta abolished SUMOylation. No SUMO binding site was identified in TRalpha LBD, suggesting that other sites in DBD could be important. Further functional studies showed that TR beta LBD mutant relieved repression on IP6, which would be in agreement with the hypothesis that SUMOylation contributes to repression. Further functional assays are being performed to confirm in vivo TRbeta SUMOylation, as well as to find binding motifs in DBDs of both isoforms. Sumo-dependent repression mediated by TRalpha and TRbeta seems to be modulated by different mechanisms. 
P114

\section{Relationship Between Nutritional and Genomic Instability Biomarkers in Short Bowell Syndrome Patients Undergoing Parenteral Nutrition}

Raquel Alves Dos Santos, Lívia María Cordeiro SimõesAmbrósio, Júlio Sérgio Marchini, Catarina Satie Takahashi, Helio Vannucchi

University of São Paulo, Brazil

Short Bowel Syndrome (SBS) generally results in severe nutrient, water and electrolyte malabsorption. Parenteral nutrition (PN) is commonly prescribed to patients with SBS and includes amounts of essential vitamins assumed to meet metabolic needs but, even under PN treatment, SBS patients present nutritional deficiencies and exhibit poor clinical outcomes. It is well known nutritional deficiencies contribute significantly to DNA damage. The present study investigated the relationship between nutritional and effect biomarkers (DNA and chromosome damage) as well as the cellular and molecular responses after PN in SBS patients before and after PN and healthy age-matched controls. Peripheral blood lymphocytes from ten healthy controls and eight SBS patients were collected before and five days after PN infusion. Comet assay and micronucleus test were employed to evaluate the extension of DNA damage. Folate and B12 serum levels were detected using the IMMULITE $\AA$ test and $\alpha$ tocopherol and retinol plasma levels HPLC methodology. Real time PCR was applied to perform the relative expression quantification from cell cycle control genes and antioxidant defense genes. Spontaneous DNA and chromosome damage were higher in SBS patients before and after PN than in controls. Interestingly, the levels of folate and B12 were higher in patients than in controls, however, tocopherol and retinol levels were significantly lower in patients than in controls. We did not observe differences of relative expression between patients and controls in cell cycle control and antioxidant defense genes. These results indicate that patients with SBS exhibit higher levels of chromosome damage than healthy controls and that PN improved the levels of folate and B12 but did not the levels of tocopherol and retinol. Likewise, at least considering the genes investigated here, molecular responses were not differently modulated after PN in SBS patients group.

Financial Support: FAPESP proc. № 2007/58409.

\section{P115}

\section{Physiological Copper Exposure in Human Mononuclear Cells Induces Changes in the Expression of Genes Encoding Copper Metabolism and Cholesterogenic Proteins}

\author{
Miriam Suazo 1,2, Talía Del Pozo ${ }^{2,3}$, Ricardo Gutiérrez ${ }^{1,2}$, \\ Cristóbal Orellana ${ }^{1,2}$, Mauricio González ${ }^{3}$ \\ ${ }^{1}$ Laboratorio de Micronutrientes, INTA-Universidad de \\ Santiago, Chile, ${ }^{2}$ Laboratorio de Nutrición Básica y \\ Epidemiología Genética, INTA-Universidad de Chile, \\ ${ }^{3}$ Laboratorio de Bioinformática y Expresión Génica, INTA- \\ Universidad de Chile
}

Introduction: Copper, an essential nutrient, is potentially toxic generating reactive oxygen species (ROS). We found that $\mathrm{Cu}-\mathrm{Zn}$ SOD1 and its chaperone (CCS) transcript were reduced in peripheral mononuclear cells (PMNCs) of individuals exposed to $10 \mathrm{mg} \mathrm{Cu}$ /day for 2 months. In addition copper excess have been shown to affect the expression of cholesterogenic genes. Here, we investigated the changes in the expression of genes encoding proteins associated with copper metabolism and cholesterol biosynthesis in human mononuclear cells.

Materials and Methods: Human cell lines of lymphocytes (Jurkat), monocytes (THP-1) and PMNCs from healthy men $(n=15)$ were exposed to sub- iso- and supra- physiological copper concentrations $(0.5,2$ and $20 \mu \mathrm{M} \mathrm{Cu}$-His) for 2-48 hours. The gene expression of ATP7A; MT2A; SOD1; Ctr1; CCS and ATOX1 (copper metabolism) and of HMG-CoA reductase; HMG CoA synthase and LDL-R (cholesterol biosynthesis) was quantified by qPCR. The copper, iron and zinc cellular content were determined by AAS.

Results: The three cellular models showed an increment of metal content after supplementation with iso- and supra-physiological copper concentrations. Under both conditions, CCS gene expression showed a significant decreased in THP1, Jurkat and PMNCs and MT2A, HMG-CoA reductase; HMG CoA synthase and LDL-R transcript increased significantly in mononuclear cells.

Conclusions: Transcriptional changes of CCS and cholesterogenic genes were induced in response to physiological range of copper concentration, suggesting a copper-dependent transcriptional regulation of these genes in human mononuclear cells. Our results indicated an association between copper and cholesterol metabolism and suggest a role in lipid-ROS related disease molecular basis. (FONDECYT Nº11070255, 1071083). 


\section{P116}

The 1258 G > A Polymorphism (rs9785023) in the Neuropeptide Y Gene is Associated with Greater Alcohol Consumption in a Mediterranean Population

\author{
Dolores Corella', José Vicente Sorli', Olga Portoles', \\ Oscar Coltell', Marisa Guillén', Carmen Saiz', \\ José I. González', Juan Javier Verdu-Castillo', \\ José María Ordovas ${ }^{3}$, Francesc Frances ${ }^{1}$ \\ ${ }^{1}$ University of Valencia, Spain, ${ }^{2}$ University Jaume \\ I-Castellon, Spain, ${ }^{3}$ Tufts University-Boston, USA
}

Introduction: Neuropeptide Y (NPY) is a neurotransmitter widely distributed in the central nervous system. Several studies have demonstrated that increases of NPY are associated with reduced alcohol intake and anxiety manifestations. The Leu7Pro $(1128 \mathrm{~T}>\mathrm{C})$ polymorphism in the NPY has been associated with alcohol consumption, but evidence is scarce. In the Spanish Mediterranean population, this variant is not polymorphic as we reported in a previous study. It is necessary to assess the prevalence of other polymorphisms in this gene as well as undertaking a screening of the exonic regions of the NPY gene in this population. Thus, our aim was to identify novel variants in the NPY and to investigate the impact of these markers, and others previously described, on alcohol consumption in this population.

Methods: We studied 911 subjects (321 men and 590 women) from the Spanish Mediterranean population. Alcohol consumption, demographic and lifestyle variables were measured. Nucleotide sequence determination and SNP analyses were carried out. We carried out a direct sequencing screening in a 50 individuals subsample.

Results: Only one exonic SNP was detected by direct sequencing (1258 G>A or rs9785023; allele frequency 0.47). From the intronic markers chosen (483 $\mathrm{A}>\mathrm{G}$ or rs 13235938, $2517 \mathrm{~A}>\mathrm{G}$ or rs4722342 and $7065 \mathrm{~A}>\mathrm{G}$ or rs4722343), only the two latter ones were polymorphic (allele frequencies 0.46 and 0.04 , respectively), and none of them were associated with alcohol consumption. However, the $1258 \mathrm{G}>\mathrm{A}$ polymorphism was associated with higher alcohol intake. This association was particularly relevant in men with high alcohol intake $(59.1 \pm 5.0 \mathrm{~g} / \mathrm{d}$ in AA as opposed to $40.6 \pm 7.5$ in the $\mathrm{G}$ carriers, $\mathrm{p}=0.022$ ) and women with moderate alcohol intake (7.3 $\pm 5.5 \mathrm{~g} / \mathrm{d}$ in AA as opposed to $4.6 \pm 3.9 \mathrm{~g} / \mathrm{d}$ in $\mathrm{G}$ carriers, $\mathrm{p}=0.048$ ).

Conclusions: The $1258 \mathrm{G}>\mathrm{A}$ polymorphism in the NPY is associated with higher alcohol consumption in the Mediterranean population.

\section{P117}

\section{Hydrolyzed Protein Enriched with Glutamine Dipeptide and Carbohydrate Improves Performance and Protects Triathletes from Muscle Damage}

Tania Pithon-Curi', Fabiano Peres ${ }^{2}$, Adriana Levada-Pires ${ }^{3}$, Marcelo Vieira', Elaine Hatanaka', María Fernanda CuryBoaventura', Renata Gorjo', Paulo Silva ${ }^{3}$, Rui Curi ${ }^{3}$, Sandro Hirabara ${ }^{1}$

${ }^{1}$ Cruzeiro do Sul University-Säo Paulo, Brazil, ${ }^{2}$ Säo Francisco University, Brazil, ${ }^{3}$ Säo Paulo University, Brazil

The aim of this study was to determine the effects of a dietary supplementation with hydrolyzed whey protein enriched with glutamine dipeptide (MGln) and maltodextrine (M) on elite triatlhetes' performance, muscle damage and inflammatory response. Nine male elite triathletes participated in this study. A randomized, double blind, crossover protocol was used. Overnight fasted subjects ingested 4 tablets of $700 \mathrm{mg}$ hydrolyzed whey protein enriched with $175 \mathrm{mg}$ of glutamine dipeptide and $50 \mathrm{~g}$ of maltodextrine diluted in $250 \mathrm{~mL}$ water. The placebo (M) consisted of 4 tablets, each containing a 700 $\mathrm{mg}$ of maltodextrine and $50 \mathrm{~g}$ of maltodextrine diluted in $250 \mathrm{~mL}$ water. Thirty minutes after the supplementation, the triathletes were submitted to an exercise test until exhaustion. During the test, the following parameters were measured: maximal oxygen consumption (VO2max), percentage of maximal oxygen consumption, and ventilatory anaerobic threshold. Blood was collected before and after the test for determination of pro-inflammatory (IL-1â, IL-6, IL-8, TNF-á) and anti-inflammatory (IL-10) cytokines in serum. Lactate dehydrogenase activity, reactive $\mathrm{C}$ protein level and total and cardiac creatine kinase activities in serum were used as indicators of muscle damage. The MGln supplementation improved the percentage of maximal oxygen consumption and ventilator anaerobic threshold. Moreover, MGln increased the duration of the test and reduced muscle damage as indicated by the decrease of lactate dehydrogenase $(30.5 \%)$, total creatine kinase (25.9\%) and muscle creatine kinase (26.8\%) activities when compared to the M group. However, the supplementation did not alter the concentration of pro- and anti-inflammatory cytokines in serum. In conclusion, the supplementation with MGln increased the performance and protected elite triathletes from muscle damage after an exhaustive exercise session.

Financial support: FAPESP, CNPq, and CAPES.

\section{P118}

Therapeutic Effects of Epigallocatechin-3Gallate (EGCG) and Silibinin on ATM Dynamics in Prostate Cancer Cell Line VCaP

Ammad Farooqi, Taskeen Mashadi

University of Lahore, Pakistan

Epigallocatechin-3-gallate (EGCG) is a major ingredient of green tea (GT) and Silibinin, the active component of Silymarin presumably hold a potential to prevent pathogenomics. Prostate cancer exacerbation is triggered by fusion transcripts formed because of genomic 
instability. An abolished ATM dynamics challenges integrity of DNA. Cell lines were analyzed to observe the effect of EGCG and Silibinin on the antiparallel expression profile of TMPRSS2-ERG and ATM In this particular study treatment of the VCaP cell line with EGCG and Silibilin recapitulated ATM expression and activity and down regulated the fusion transcript expression.

\section{P119 \\ Effect of Eight Polymorphisms on Weight Loss Outcomes in Overweight/Obese Caucasian Adults}

\author{
Janetta Harbron', Monique Zaahl', Maritha Kotze, \\ Lize van der Merwe ${ }^{4}$, Marjanne Seneka/ ${ }^{1}$ \\ ${ }^{1}$ Division of Human Nutrition, University of Cape Town, \\ ${ }^{2}$ Department of Genetics, University of Stellenbosch, \\ ${ }^{3}$ Department of Pathology, University of Stellenbosch, \\ ${ }^{4}$ Biostatistics Unit, Medical Research Council, South Africa
}

Introduction: Genetic screening to improve/personalize obesity treatment outcomes is available on the market despite the lack of conclusive evidence in this regard. The aim of this study was to investigate the association between genotype (eight polymorphisms) and weight loss outcomes following a weight loss programme.

Methods: $\mathrm{N}=88$ adult volunteers $\left(\mathrm{BMI}>27 \mathrm{~kg} / \mathrm{m}^{2}\right)$ participated in a six-month conservative weight loss programme that included dietary, physical activity and behavioral components. SNPs investigated: FABP2 Ala54Thr, INSIG2 rs7566605, FTO rs1421085, FTO rs17817449, ADRB3 Trp64Arg, ADRB2 Arg16Gly, GNB3 C825T, PPAR 2 Pro12Ala. Baseline and follow-up life-style assessments included physical activity, general psychological health (GHQ), selfesteem, depression and eating behaviour (restraint, disinhibition and hunger). Statistical analyses involved mixed effects linear models that were adjusted for gender and compliance to the weight loss programme.

Results: The mean weight loss over six-months was $7.8 \mathrm{~kg}$ or 8.6\%. Mutant Gly16Gly subjects of ADRB2 lost less weight $(\mathrm{p}=0.0087)$ over the first month. Subjects with a mutant Gly16-allele and mutant C-allele of INSIG rs7566605 lost less weight over six months than other allelic combinations of these two polymorphisms. Wild-type TT subjects of FTO rs17817449 lost more weight over the first two months $(\mathrm{p}=0.0398)$. An improvement in dietary restraint (avoidance of fattening food) $(\mathrm{p}=0.0238)$, disinhibition $(\mathrm{p}=0.0331)$, emotional $(\mathrm{p}=0.0017)$ and habitual $(\mathrm{p}=0.0150)$ disinhibition was associated with a more pronounced weight loss over six months in wild-type TT subjects of FTO rs1421085. Improved GHQ and BDI was associated with a more pronounced weight loss in mutant Thr54Thr subject of FABP2 ( $\mathrm{p}=0.0261)$ and wild-type CC subjects of GNB3 ( $\mathrm{p}=0.0291)$, respectively.

Conclusion: Genetic screening should only be considered if it is evidence based and has clinical utility. Integration of the results with existing evidence indicates that ADRB2 Arg16Gly polymorphism may be included in a genetic screen for weight loss. The novel findings need to be confirmed in further research.

\section{P120}

Association Between Antioxidant Status and MnSOD Ala-9Val Polymorphism in Trained Male Athletes (Rugby Players) and Sedentary Male Students Controlled for Antioxidant Intake: An Exploratory Study

\author{
Maria Seele ${ }^{1}$, Maritha Kotze ${ }^{2}$, Theo Nell', \\ Marjanne Seneka/ ${ }^{3}$ \\ ${ }^{1}$ Department of Physiological Sciences-Stellenbosch \\ University, ${ }^{2}$ Department of Pathology, Stellenbosch \\ University, ${ }^{3}$ Department of Human Biology, University of \\ Cape Town
}

Introduction: Exercise may result in the increased generation of free radicals, increasing dietary antioxidant needs, although those who undergo regular training have been reported to develop improved antioxidant capacity. As the presence of the MnSOD Ala allele has been associated with increased oxidative stress, it may be of value to consider this polymorphism when assessing antioxidant needs of rugby players. The aim of this study thus was to investigate the association between total plasma antioxidant status, p-vitamin $\mathrm{C}$ and carotenoid levels and the MnSOD Ala-9Val (-28C T) polymorphism in trained male athletes (rugby players) and sedentary male students while controlling for dietary intake of the major antioxidants.

Methods: Plasma vitamin C, carotenoid levels, total plasma antioxidant status (ORAC assay) and MnSOD Ala-9Val genotype were determined in trained rugby players $(n=76)$ and sedentary male subjects $(n=39)$. Dietary intake of antioxidants was assessed using a FFQ validated for the purposes of this study against dietary records and biomarkers.

Results: Rugby players had significantly higher plasma vitamin C $((p=0.005)$ and carotenoid $(p=0.001)$ levels and tended to have higher total antioxidant status compared to sedentary students when controlled for dietary intake. MnSOD Ala-9Val genotype did not differ significantly between the two groups, with the prevalence of the Ala/Ala homozygote for the total group being $26.1 \%$, the Val/Ala heterozygote $50.4 \%$ and the Val/Val homozygote $23.5 \%$. MnSOD Ala-9Val genotype was not associated with the total antioxidant capacity, p-vitamin $\mathrm{C}$ or $\mathrm{p}$-carotenoid levels in either of the two groups or the combination of the two groups.

Conclusion: Rugby players undergoing regular exercise training had a more satisfactory antioxidant status compared to sedentary students. The lack of association between antioxidant status and MnSOD Ala-9Val genotype may indicate that this polymorphism does not need to be considered when determining the antioxidant needs of young males, either sedentary or rugby players. 


\section{P121}

\section{Slc27a2 Expression in Peripheral Blood Mononuclear Cells as a Molecular Marker for Overweight Development Induced by Cafeteria Feeding}

\author{
Paula Oliver ${ }^{1}$, Antoni Caimari ${ }^{1}$, Wendy Rodenburg ${ }^{2}$, \\ Jaap Keijer ${ }^{2}$, Andreu Palou ${ }^{1}$ \\ ${ }^{1}$ University of the Balearic Islands and CIBER de \\ Fisiopatología de la Obesidad y Nutrición (CIBERobn), \\ Palma de Mallorca, Spain, ${ }^{2}$ Wageningen University, \\ Wageningen, The Netherlands
}

Introduction: Peripheral blood mononuclear cells (PBMC) are readily accessible biological material and a potential tissue to assess biomarkers of biological effects using gene expression studies. Obesity is becoming the most common health problem of the $21 \mathrm{st}$ century, being dietary intake an important determinant of this pathology and its related complications. To prevent obesity, it would be of interest to have early markers of body weight gain.

Methods and Materials: Our objective was to identify in PBMC genes involved in energy homeostasis which could be good markers of overweight development. For that purpose, we evaluated, using whole genome microarray analysis, the gene expression in PBMC of normoweight and diet-induced obese (cafeteria-fed) Wistar rats.

Results: Microarray analysis showed 566 genes differentially expressed between normoweight and cafeteria-fed rats. Of these, 35 genes were particularly involved in energy homeostasis. The gene with the biggest fold change was the "solute carrier family 27 (fatty acid transporter), member 2" (slc27a2), which is implicated in lipid biosynthesis and fatty acid degradation. Scl27a2 was 33 fold overexpressed in cafeteria-fed rats compared to normoweight rats. This result was confirmed by Q-PCR, although the over-expression was smaller (6 fold). Moreover, the increase in slc27a2 expression in PBMC of cafeteria-fed rats from 2 to 6 months of age paralleled the increase in body weight.

Conclusion: The progressive over-expression of slc27a2 in PBMC of cafeteria-fed rats, as body weight increases, suggests this gene as an early marker of overweight development related to the intake of a hyperlipidic diet.

Funding: CIBER de Fisiopatología de la Obesidad y Nutrición is an initiative of the ISCIII. The groups of A.P. and J.K. are members of the European Research Network of Excellence NuGO (The European Nutrigenomics Organization).

\section{P122 \\ Differences in Circadian Rhythms of Wrist Temperature Between Obese and Normal Weight Women. Associations with Metabolic Syndrome Features}

\author{
M.D. Corbalán-Tutau1', J.A. Madrid1', J.M. Ordovás ${ }^{2,3}$, \\ F. Nicolás ${ }^{4}, M$. Garaulet ${ }^{1,2}$ \\ ${ }^{1}$ Department of Physiology, Faculty of Biology, University \\ of Murcia, Murcia, Spain, 2Jean Mayer US Department of \\ Agriculture Human Nutrition Research Center on Aging, \\ Tufts University School of Medicine, Boston, MA, ${ }^{3}$ The \\ Department of Epidemiology and Population Genetics \\ Centro Nacional Investigación Cardiovasculares (CNIC) \\ Madrid, Spain, ${ }^{4}$ Department of Nuclear Medicine Arrixaca \\ Hospital, Murcia, Spain
}

Circadian rhythm of core temperature is associated with widespread effects throughout the body. The aim was to characterize the circadian rhythmicity patterns in obese and normal-weight women by measuring wrist temperature (WT). Daily patterns of cortisol, melatonin and different metabolic syndrome (MetS) features were also analyzed in an attempt to clarify the pathological substratum of circadian rhythmicity in obesity.

The study was conducted in 70 women, normal-weight women $(\mathrm{n}=20)$ (mean age: $38 \pm 11)$ (mean BMI: $\left.22 \pm 2.6 \mathrm{~kg} / \mathrm{m}^{2}\right)$ and obese women $(\mathrm{n}=50)$ (mean age: $42 \pm 10)$ (mean BMI: $\left.33.5 \pm 3.2 \mathrm{~kg} / \mathrm{m}^{2}\right)$. Skin temperature was measured during three days every $10 \mathrm{~min}$ with the "Thermochron iButton". Rhythmic parameters were obtained using an integrated package for temporal series analysis "Circadianware ${ }^{\circledR}$ ".

Obese women displayed significantly lower mean WT (34.1 \pm 0.3 ) with a more flattened pattern, a less quality rhythm and a higher intraday variability (IV). Three segments in the chronogram differed between obese and normal-weight women: a) after getting up, b) before the times of light off, c) secondary WT peak in the postprandial time (P2). WT Rhythmicity characteristics were related with MetS features and energy intake-related proteins such as plasma leptin, and ghrelin. Circadian markers, cortisol and melatonin, were also associated with WT rhythmicity.

In conclusion, obese women displayed a less quality WT circadian rhythm than normal-weight. These circadian changes were related to women metabolic status towards a higher MetS. The measurement of WT could be an interesting tool to evaluate chronodisruption in obese patients in clinical practice. 
Journal of

Nutrigenetics

Nutrigenomics
Addendum to:

Published online: November 17, 2010

J Nutrigenet Nutrigenomics 2010;3:49-124

\section{P123 \\ Metabolomics, Nutrition and Polyphenols: Non-Targeted Analysis of Urinary Metabolome Modifications after Intake of Almond-Polyphenols as Poweful Approach to Identify New Nutritional Biomarkers \\ Rafael Llorach 1, 3 , Ignacio Garrido ${ }^{2}$, Maria Monagas 2, 3, Mireia Urpi-Sarda 1,3, Sara Tulipani 1,3, Begoña Bartolome 2,3, Cristina Andrés-Lacueva ${ }^{1,3}$ \\ ${ }^{1}$ Nutrition and Food Science Department, XaRTA, INSA, ${ }^{2}$ Instituto De Fermentaciones Industriales (CSIC), \\ ${ }^{3}$ INGENIO-CONSOLIDER Program, Fun-C-Food, CSD2007-063, \\ Ministry of Science and Innovation, Spain}

Almond skin is a source of polyphenols, mainly procyanidins. The health effects of these polyphenols depend on their bioavailability implying tissue metabolites and gut microbial metabolites. An important challenge of the nutri-metabolomics is to identify new biomarkers that allow monitoring the intake of dietary phytochemicals (food metabolome) and finally relate them with the expected biological effects (endogenous metabolome). The modifications occurred in urine metabolome during the $24 \mathrm{~h}$ after consumption of almond-skin extract were studied. Twenty-four healthy subjects were randomized into two group $(\mathrm{n}=12$, placebo or dosed). Placebo-group ingested microcrystalline cellulose ( $4 \mathrm{~g}, 10$ capsules) whereas dosed-group ingested almond-skin extract (3.5 g, 10 capsules). Urine samples were collected 0-2, 2-6, 6-10 and 10-24 h after consumption. Samples were analyzed by HPLC-q-ToF (Applied-Biosystems) followed by multivariate data analysis. To evaluate possible analytical artefacts several QCs were carried-out. Identification was performed using public databases (KEGG, HMDB) and in-house database focused on polyphenols metabolites and MS/MS experiments. Urinary metabolic profiling showed a clear discrimination between both test meals at the four assayed times. Kinetic patterns for different biomarkers related with almond-skin consumption were detected. Host metabolites were excreted within the first $10 \mathrm{~h}$ and others such as microbial-polyphenol metabolites were present at $10-24 \mathrm{~h}$ period. A total of 34 polyphenols metabolites have been identified including host metabolites of flavanols monomers as well as colonic microbiota polyphenols such as glucuronide and sulfate derivatives of phenolics acids and hydrophenylvalerolactones. Several of these metabolites have been related for the first time as markers of procyanidins intake. These results confirm that metabolomics is a powerful tool that allows to characterize the food metabolome and to explore the metabolism impact of dietary components.

\section{P124 \\ Identification of Hepatic Transcription Biomarkers for Insulin Resistance by the Level of RNA Expression and the Mode-of-Action by Network Identification in High-Fat Diet Induced Obese C57bl/6J Mice}

\author{
Eunjung Kim ${ }^{1}$, Hyoung-Sam Heo ${ }^{2}$, Eun-Young Kwon ${ }^{3}$, \\ Su-Kyung Shin ${ }^{3}$, Seon-Min Jeon ${ }^{3}$, Seung-Won Seo ${ }^{2,4}$, \\ Cheol-Goo Hur ${ }^{2}$, Myung-Sook Choi ${ }^{3}$ \\ ${ }^{1}$ Catholic University of Daegu, ${ }^{2}$ Korea Research Institute of \\ Bioscience and Biotech, ${ }^{3}$ Kyungpook National University, \\ ${ }^{4}$ University of Science and Technology, Seoul, Korea
}

Insulin resistance (IR) refers to reduced sensitivity of insulin target tissues (liver, skeletal muscle, adipose tissue) to insulin. Recent reports suggest that IR, not obesity, is the best predictor of metabolic syndrome because some obese individuals remain relatively insulin-sensitive and metabolically healthy, whereas many lean individuals are insulin resistant and at high risk for obesityrelated complications. In this study, we fed C57BL/6J mice with high-fat diet (HFD) for 0, 2, 4, 6, 8, 12, 20, 24 weeks and systemically analyzed time course specific hepatic transcriptome profile by both differentially expressed genes (DEG) and mode-of-action by network identification (MNI) algorithm to identify IR biomarkers. Biochemical data showed that the levels of fasting blood and plasma glucose and IR related hormones such as insulin, leptin in HFD group became significantly higher than those of normal diet (ND) group after 16-20 weeks of HFD feeding. Impaired glucose tolerance was also observed in HFD group after 16 weeks of HFD feeding. In accordance with this, when we performed network alignment between protein-protein interaction (PPI) network of previously reported 189 IR biomarkers (IRB) and PPI network of HFD induced common genes (proteins) identified by both DEGs and MNI (DEGs \& MNI), we observed that as duration of HFD feeding period was extended, overlapping of the two networks became more significant and moreover DEGs \& MNI network moved toward core of the IRB network. We also identified peroxisome proliferator-activated receptor delta and gamma, protein tyrosine phosphatase 1, phosphoenolpyruvate carboxykinase 1, and hydroxysteroid 11-beta dehydrogenase 1 as key role proteins in this analysis and time shift of these key role proteins during HFD feeding. Taken together, these data suggest that application of both DEGs and MNI and PPI network analysis can be a powerful tool to analyze gene transcripts obtained from microarray analysis for searching possible key role genes or proteins.

\section{KARGER}

두 2010 S. Karger AG, Basel

Fax +41 613061234 E-Mail karger@karger.ch www.karger.com www.karger.com/jnn 


\title{
P125 \\ Soy Intake Benefits Glycemic Control in Healthy Individuals Depending on Estrogen Receptor Polymorphisms
}

\author{
Jose C.E. Serrano ${ }^{1}$, David De Lorenzo ${ }^{1}$, Alberto Espinel ${ }^{2}$, \\ Jordi Boada ${ }^{1}$, Hugo Gonzalo ${ }^{1}$, Margot Roig Marin-Yoseli ${ }^{1}$, \\ Marco Antonio Delgado ${ }^{2}$, Reinald Pamplona ${ }^{1}$, \\ Manuel Portero-Otin ${ }^{1}$ \\ ${ }^{1}$ IRB Lleida School of Medicine, University of Lleida, \\ ${ }^{2}$ I+D, Grupo Leche Pascual, Madrid, Spain
}

Materials and Methods: An interventional study was designed, with 108 healthy participants being assigned to either no intake, and daily $250 \mathrm{ml}$ or $500 \mathrm{ml}$ supplementation groups ( $\mathrm{n}=$ 30-38 per group) for 2 months. Selected variables related to glycemic control, lipid metabolism, as well as to cardiovascular risk were recorded. Bucal swabs were used for analyses of the GPER, ESR2 (both estrogen receptors) and SLC2A9 (glucose transporter) genes.

Results: Multivariate analyses show that before dietary intervention, polymorphisms in either GPER, ESR2 and SLC2A9 were associated to significant baseline differences in HbAlc $(\mathrm{p}<0.032$; $\mathrm{p}<0.021 ; \mathrm{p}<0.025$, respectively). ESR2 polymorphism was associated to significant differences in fasting glycemia $(\mathrm{p}<0.041)$ and SLC2A9 SNP to triacylglyceridemia $(p<0.015)$. Soy intake, withoud taking into consideration genetic influence, led to significant decreases in HbAlc $(\mathrm{p}<0.040)$ demonstrating a better glycemic control. However, this effect showed a near-significant interaction with GPER and ESR2 polymorphisms $(p<0.06)$. This association improved after gender adjustment $(\mathrm{p}<0.009$ for Gpr30). SLC2A9 polymorphisms were associated to a better response to soy intake in terms to OGTT $(\mathrm{p}<0.032)$. Most interestingly, when considering double interactions between isoflavone receptors, significant differences in the effects of soy intake were very evident ( $p<0.01$ for HbAlc, $\mathrm{p}<0.03$ for OGTT, $\mathrm{p}<0.05$ for triacylglyceridemia and $\mathrm{p}<0.001$ for antioxidant capacity).

Conclusions: (i) Soy intake leads to a beneficial effect in glycemic metabolism in healthy individuals and (ii) the improvement degree is strongly dependent on SNP of both estrogen cellular receptors as well as on glucose transport, overall suggesting the need for nutrigenomic adjustment of population before nutritional intervention studies. 


\section{Nutrigenetics Nutrigenomics}

\author{
Abdalla, D.S.P. 77, 80 \\ Aceto, G.M. 115 \\ Aguilera, C.M. 108 \\ Al-Massadi, O. 76 \\ Alberich, R.S. 95 \\ Alburquerque-Béjar, J.J. 78, \\ 112 \\ Alegría, A. 71 \\ Amil-Diz, M. 76 \\ Amiot, M.-J. 99 \\ Anchuelo, A.C. 111 \\ Anglés, N. 76, 83 \\ Arbo, I. 98 \\ Ardèvol, A. 86, 96 \\ Ares, R. 103 \\ Aresti, G.A. 100 \\ Arner, P. 66, 97 \\ Arós, J.S.-S.F. 83 \\ Arroyo-Pardo, E. 72, 74 \\ Arruda, S.F. 73 \\ Arthur, T. 110, 112 \\ Astrup, A. 104 \\ Astudillo, A. 111 \\ Atzori, L. 87 \\ Ayo, J. 70 \\ Azcona-Sanjulian, C. 107 \\ Azevedo, M.J. 90 \\ Azzini, E. 96
}

Baeza, C. 72, 74

Balsinde, J. 111

Barale, R. 75, 94

Baraza, J.C. 78

Barbáchano, A. 91

Barbera, R. 71

Bastida, S. 79, 82

Beal, F.L.R. 73

Beghin, L. 103

Béliard, S. 69

Belotto, M.F. 88

Benavides, L.G. 97

Bened, J. 79

Benito, P. 103

Bento de Lima, C. 89

Bernal, C. 102

Bertoncini, S. 72, 74

Blanco-Rojo, R. 72, 74

Blay, M. 86, 96

Boada, M. 76

Bogl, L.-H. 79

Bolea, I. 76

Bonilla, F. 91

Boqué, N. 89, 107
Borelli, P. 97

Borges, M.C. 97

Bouchard, C. 56

Bouchard-Mercier, A. 82

Bouloumie, A. 97

Brattbakk, H.-R. 98

Bressan, J. 106

Brüne, B. 77

Bueno, G. 109

Bueno, M. 109

Bueno, O. 109

Buonomo, T. 80, 93

Burdge, G. 62

Bustos, M. 116

Butigan, R. 115

Cabral, M. 69

Cáceres, P.M. 116

Caimari, A. 121

Calabriso, N. 99

Calderón-Enríquez, R.E. 104

Calvenzani, V. 76

Camargo, A. 114

Campa, D. 75, 94

Campión, J. 55, 90, 101, 107, 108

Campos-Góngora, E. 103,

104

Canali, R. 96

Cañete, R. 108

Cansell, M. 114

Canzian, F. 75, 94

Caradonna, F. 94

Cardona, E.G. 97

Carluccio, M.A. 80, 85, 93, 99, 110

Carpéné, C. 64, 87

Carracedo, J. 74

Carrai, M. 75, 94

Carrasco-Benso, M.P. 78, 112

Carrasco, P. 84

Carreres, J.E. 98

Casado, N.D. 114

Casanueva, F.F. 76

Casillas, R. 84

Castañer, O. 83

Castelao, C. 76

Castell-Auví, A. 86, 96

Catalán, Ú. 75, 83

Cataldo, R. 105, 111

Catanzaro, I. 94

Cedó, L. 96

Chaible, L.M. 92
Chaves, G.A.T. 73

Chmurzynska, A. 81, 113

Cho, S.-J. 105

Cho, Y.-Y. 77

Choi, M.-S. 77, 105

Churruca, I. 70, 100

Ciavardelli, D. 96

Cilla, A. 71

Ciudad, C.J. 91

Cobián, T.A.G. 97

Cobos, L.T. 98

Coltell, O. 84, 119

Combe, N. 114

Comes, G. 76

Conradie, K. 85

Contreras, R. 108

Contu, P. 87

Cooney, G. 90

Corbalán-Tutau, M.D. 121

Corda, E. 87

Corella, D. 82, 83, 84, 119

Corrales, F.J. 67

Costa, A.G.V. 106

Couedelo, L. 114

Covas, M. 84

Covas, M.-I. 83

Crocco, P. 75

Crujeiras, A.B. 76

Curi, R. 88, 89, 90, 97, 117, 119

Cury-Boaventura, M.F. 119

Cvoro, A. 117

Dagli, M.L.Z. 92

Dahlman, I. 97

Daleprane, J. 77, 80

Daviaud, D. 87

David, R.O. 79

De Almeida Siqueira, E.M. 73

De Caterina, R. 59, 80, 85, 93, 99, 110

De Conti, A. 92

De La Cruz, R. 104

de Lorgeril, M. 76

De Oliveira Andrade, F. 92

De Rango, F. 75

DeHenauw, S. 103

Dehne, N. 77

del Carmen Ochoa, M ${ }^{\mathrm{a}} 107$

Del Pozo, T. 118

Delgado-Casado, N. 113

Delgado-Lista, J. 74, 113

Dentin, R. 70

Deram, S. 110
Di Gioacchin, M. 115

Di Pietro, N. 96

Di Silvestre, S. 96

Di Tomo, P. 96

Dias De Paula, T. 95

Díaz, L. 103

Do, G.-M. 77

Durand, T. 114

Erasmus, R. 102

Erasmus, R.T. 102

Escorihuela, R.M. 76

Espinosa-Salinas, I. 71

Estivill, X. 111

Estruch, R. 83, 84

Fanampe, B. 102

Farooqi, A. 80, 119

Farré, J.A.L. 116

Farsky, S. 89

Federico, C. 94

Ferguson, L.R. 68

Fernandes de Oliveira, F. 73

Fernández-Castillejo, S. 75

Fernández-Galilea, M. 106, 109

Fernández-Irigoyen, J. 67

Fernández, L. 76

Fernández-Quintela, A. 70

Fernandez, S. 83

Ferrari, M. 103

Fiamoncini, J. 90

Fitó, M. 83

Fock, R. 97

Fontelles, C. 92

Formiguera, X. 106

Fornari, T. 92

Fortes de Valencia, F. 73

Frances, F. 119

Frazzatto, E. 110

Frongia, P. 87

Frota de Farias, R. 117

Fuentes, F. 113, 114

Fujii, T. 97

Fujisaka, S. 86

Fujiwara, C. 112

Fullin, G. 95

Galassi, S. 87

Galbete, C. 107, 108

Garaulet, M. 78, 101, 106, 112, 121

García, A.T. 116

García, C. 70

\section{KARGER}

(C) 2010 S. Karger AG, Basel

Fax +4161306 1234

E-Mail karger@karger.ch

www.karger.com 
García-Díaz, D. 108

Garcia, M. 91

García-Nebot, M.J. 71

García-Rios, A. 74

García-Risco, M.R. 92

Gastaldelli, A. 110

Gawecki, J. 81

Gesteiro, E. 82

Giardinelli, A. 96

Gibney, M.J. 58

Gil, A. 60, 108

Gil-Campos, M. 108

Gil de Gómez, L. 111

Gill, T. 89

Giné, L.C. 86

Giorgio, M. 76

Godin, G. 82

Gómez-Abellan, P. 101

Gómez, P. 74

Gómez-Santos, C. 101, 106

González, A. 105

González-Castejón, M. 71

González, D. 105

González, J.I. 84, 119

González, M. 102, 118

González-Martínez, B.E. 103, 104

González-Vallinas, M. 72, 92

Gorjo, R. 119

Gouranton, E. 99

Gratacos, M. 111

Grau, K. 104

Grès, S. 87

Groos, J.L. 90

Guazzelli, I. 110

Gueis, T. 77

Guillen-Grima, F. 108

Guillén, M. 84, 119

Guillen, M.L. 82

Gutiérrez, R. 118

Guy, A. 114

Halpern, A. 112

Hansen, T. 104

Harbron, J. 120

Hasan, F. 89

Hassan, M. 102

Hassen, M. 102

Hatanaka, E. 88, 119

Heras, M. 83

Hernandez-Morante, J.J. 106

Hernández, P.E.S. 97

Hidalgo, J. 76

Hirabara, S. 89, 90, 117, 119

Ho-Urriola, J. 105

Hodgson, M.I. 105

Hoekstra, T. 85

Holecek, M. 94

Holst, C. 104

Hu, F.B. 65
Hur, C.-G. 77

Iffiú-Soltész, Z. 64

Iglesias, T.G. 97

Ikegaki, M. 77, 80

Izquierdo-Pulido, M. 91

Jacob, P. 97

Jacques, H. 81

Jeon, S.-M. 77, 105

Jiménez, F.P. 114

Jiménez-Salas, Z. 103, 104

Johansen, B. 98

Jordão Jr, A.A. 92

Junien, C. 65

Kähönen, M. 69

Kanatani, Y. 86

Kandil, M. 75

Kang, J.X. 60

Kaprio, J. 79

Keijer, J. 121

Kendrovski, V. 115

Konayashi, M. 86

Kotze, M. 120

Kovarik, M. 94

Kuan, R.Y.W. 94

Kulseng, B. 98

Kwon, E.-Y. 77, 105

Laakso, M. 87

Laaksonen, M. 69

Labayen, I. 103

Lairon, D. 65

Lamarche, B. 82

Lambertucci, R.H. 117

Lamuela-Raventós, R. 83

Landrier, J.-F. 99

Langaas, M. 98

Langin, D. 57

Larrarte, E. 103, 106

Larriba, M.J. 91

Lasa, A. 70, 100, 102

Laurencikiene, J. 97

Lavigne, C. 81, 89

Leandro, C.G. 117

Lehtimäki, T. 69

Leis, R. 108

Leslie, S. 90

Levada-Pires, A. 119

Li, N. 70

Lillycrop, K. 62

Lima, T. 89, 90

Lindberg, F. 98

Lisanti, S. 61

Lista, J.D. 114

Loos, R.J.F. 64

López-Cabanillas, M. 103, 104

López-Mínguez, J. 78, 112

López-Miranda, J. 74, 113
López-Parra, A.M. 72, 74

Lorente-Cebrián, S. 106

Lovegrove, J. 59

Luján, J.A. 101

Macarulla, M.T. 100

Macaya, C. 116

McDonald, I. 104

Machado de Almeida, E. 73

McLeod, R. 89

Madrid, J.A. 101, 121

Magdalon, J. 88

Maiz, A. 104, 111

Malezet, C. 99

Malinowska, A. 81, 113

Mancini, M. 112

Maranghi, M. 79

Maraninchi, M. 69

Marçal, A. 90

Marchini, J.S. 118

Marcos-Gómez, B. 116

Marcotorchino, J. 99

Marette, A. 81, 89

Margareto, J. 103, 106

Mariani-Costantini, R. 115

Mariman, E.C.M. 62

Marin, C. 74

Marín, F. 71

Mariscal, F.M.G. 114

Marques De Matos-Neto, E. 95

Marti, A. 107, 108

Martín-Venegas, R. 69

Martinelli, R. 80, 93

Martines, G. 80, 85

Martínez-Anso, E. 116

Martínez de la Piscina, I. 116

Martínez-González, M.Á. 107, 108

Martinez Larrad, M.T. 109, 111, 116

Martínez, J.A. 55, 78, 90, 101, 104, 106, 107, 108, 109, 116

Martínez, M.Á. 84

Martínez, P.P. 114

Martisová, E. 107

Masaki, M. 85, 94

Mashadi, T. 119

Massaro, M. 80, 85, 93, 99, 110

Mateos, J.A. 109

Mathers, J.C. 61

Matsha, T. 102

Mejhert, N. 97

Melo, M.E. 112

Menrad, H. 77

Mercader, J. 64, 87

Mereu, A. 87

Mereu, R. 87

Merino, A. 74

Mikkilä, V. 69
Milagro, F.I. 55, 90, 101, 107, 108

Milner, J.A. 67

Miranda, J. 70, 100

Miranda, J.L. 114

Mitjavila, M.T. 84

Modrego, J. 116

Moleres, A. 107, 108

Molina, S. 72, 92

Mononen, N. 69

Montinari, M.R. 99

Morales, A.S.M.Z. 97

Morales-Falo, E. 112

Moreira, A.R.S. 79

Moreira, D.S. 73

Morello, J. 83

Moreno-Aliaga, M.J. 66, 78 106, 108, 109, 116

Moreno, C.S. 78, 112

Moreno, F.S. 92

Moreno, J.J. 69, 84

Moreno, L. 103, 109

Moschetta, A. 68

Moschonis, G. 103

Motta, S. 94

Muniz, F.J.S. 82

Muñoz, A. 91

Muñoz, J. 67

Muntoni, S. 87

Murtra, P. 76

Mutch, D.M. 63

Nachbar, R.T. 117

Nagamine, M.K. 92

Najeh, H. 75

Napolitano, F. 115

Naranjo, C. 104

Näslund, E. 97

Navarro, $\mathrm{M}^{\mathrm{a}}$ T. 98

Nell, T. 120

Nicolás, F. 121

Nicolay, A. 69

Noé, V. 91

Nogueira, J.P. 69

Oleaga, C. 91

Oliver, P. 121

Olza, J. 108

Ong, T.P. 77, 80, 92

Oppert, J.-M. 104

Ordovás, J.M. 78, 82, 84, 101, $112,119,121$

Orellana, C. 118

Ortega-Azorin, C. 84

Ortega, F. 103

Pallarès, V. 86, 96

Pálmer, H. 91

Palou, A. 121

Pandolfi, A. 96 
Pantaleão, L.C. 95

Pardo, M. 76

Park, H.-J. 77, 105

Park, Y.B. 105

Passarino, G. 75

Paternain, L. 107

Pedersen, O. 104

Peltonen, M. 87

Perconti, S. 115

Pereira, F. 91

Peres, F. 119

Pérez-Granados, A.M. 72, 74

Pérez-Jiménez, F. 74, 113

Pérez-Martinez, P. 74, 113

Pérez-Matute, P. 78, 106, 109

Pérez, N.R. 111

Pérusse, L. 56, 82

Petroni, K. 76

Pettersson, A. 97

Pieters, M. 85

Pietilinen, K.H. 79

Pilu, R. 76

Pinent, M. 86, 96

Pinet, S. 114

Pinillos, S. 109

Pipino, C. 96

Pithon-Curi, T.C. $88,117,119$

Pons, L. 83

Pons-Llecha, L. 75

Portillo, M.P. 70, 100, 102

Portolés, O. 83, 119

Poutanen, K. 58

Prasad, C. 93

Prieto-Hontoria, P.L. 78, 106, 109

Prieto, J. 67, 116

Pusceddu, P. 87

Quintanilla-Cantú, A. 103

Quintero, P. 108

Raiconi, G. 115

Raitakari, O. 69

Ramirez, B. 76

Ramírez de Molina, A. 71, 72, 92

Ramírez, M J. 107

Ramirez, R. 74

Ramos, R. 72

Reglero, G. 71, 92

Reinhardt, H. 112

Rendo-Urteaga, T. 107

Represa, J.F. 111, 116

Rezende, T. 95

Riollet, C. 99

Rioux, L.-E. 89

Rissanen, A. 79

Rodenburg, W. 121

Rodrigo, A. 98

Rodrigues, H. 88,89
Rodríguez-Casado, A. 71, 72

Rodríguez, V.M. 100

Rogero, M. 97

Rojas, J.M. 91

Romier, B. 99

Rosa, F.R. 73

Rose, G. 75

Ross, S.A. 62

Rudkowska, I. 81

Ruiz-Gutiérrez, V. 83, 84

Ruiz, J. 103

Rupérez, A.I. 108

Rydén, M. 97

Sabbioni, E. 115

Saccone, S. 94

Sain, J. 102

Saiz, C. 84, 119

Salas, J. 84

Salazar, L. 80

Salinas, M ${ }^{a}$ I.E. 72

Sánchez-Muniz, F.J. 79

Sánchez-Quiles, V. 67

Santamaría, E. 67

Santos, A. 112

Santos, J.L. 63, 104, 105, 111

Santos, R.A.D. 118

Santoyo, S. 92

Saris, W.H.M. 57, 104

Sato, F. 89

Saus, E. 111

Schmid, T. 77

Schweiger, M. 100

Scoditti, E. 80, 85, 93, 99, 110

Seele, M. 120

Senda, S. 86

Senekal, M. 120

Seoane, L.M. 76

Serrano, E.M.Y. 113, 114

Serrano Ríos, M. 109, 111, 116

Shidoji, Y. 85, 94

Shin, S.-K. 77

Sievänen, H. 69

Silva, P. 119

Silveira, L.D.R. 117

Siman, P. 94

Simões-Ambrósio, L.M.C. 118

Simón, E. 100

Simopoulos, A.P. 55

Sispera, L. 94

Smalley, S.V. 105

Sola, R. 75, 83

Solé, A. 91

Soler, C. 71

Solís-Pérez, E. 103, 104

Sorensen, T.I.A 104

Sorli, J.V. 84, 119

Spiroski, I. 115

Stachowiak, M. 113

Stanèáková, A. 87
Steemburgo, T. 90

Storelli, C. $80,85,99,110$

Storniolo, C. 84

Suazo, M. 118

Sutto, S.E.T. 97

Suzuki, H. 86

Tagliaferri, R. 115

Takahashi, C.S. 118

Taskinen, M.-R. 79

Tasset-Cuevas, I. 113

Thabuis, C. 99

Tinelli, A. 99

Tirapegui, J. 95, 97

Titta, L. 76

Tobe, K. 86

Togashi, M. 117

Tojo, R. 108

Toledo, J. 108

Tolonen, S. 69

Tomás, D. 98

Tonelli, C. 76

Torres, L.G. 79

Toufektsian, M.-C. 76

Trayhurn, P. 66

Tunez, I. 113

Tuomilehto, J. 87

Turner, N. 90

Twardowska-Rajewska, J. 81

Unzeta, M. 76

Urakaze, M. 86

Urata, M. 94

Usui, A.B. 86

Uusitupa, M. 87

Vaique, E. 114

Valdecantos, M.P. 78

Valéro, R. 69

van der Merwe, L. 102, 120

Vannucchi, H. 92, 118

Vaquero, M.P. 72, 74

Vargas, O.E.F. 97

Vaysse, C. 114

Velasco, M.V. 79

Velázquez-Arellano, A. 56

Verdu-Castillo, J.J. 119

Verginelli, F. 115

Vergotine, Z. 102

Viadel, B. 98

Vieira, M. 119

Viikari, J. 69

Vila, A.A. 95

Villalba, J.M. 113

Villares, S. 110

Vinolo, M.A.R. 88, 89, 97,

117

Virgili, F. 96

Vohl, M.-C. 81, 82

von Zglinicki, T. 61
Vuletic, A. 115

Wan, J.-B. 60

Wanecq, E. 64

Wang, J. 70

Waterland, R.A. 61

Webb, P. 117

Yako, Y. 102

Yamada, M. 97

Yamazaki, Y. 86

Yap, R.W.K. 85

Yates, A.A. 58

Zaahl, M. 120

Zabena, C. 109, 111, 116

Zamora, R.M.V. 95 


\section{Erratum}

In the Abstract P038, 'The effect of the 4G/5G polymorphism on PAI-1act in a black South African population', published in vol. 3, issue 2-3, 2010 on p. 85 of 'Abstracts of the 4th Congress of the International Society of Nutrigenetics/Nutrigenomics', not all the authors were listed. The complete list and affiliations are as below:

K.R. Conradie', T. Hoekstra ${ }^{3}$, M. Pieters' ${ }^{1}$, W. Stonehouse ${ }^{4}$, A. Kruger², J. Jerling ${ }^{1}$

${ }^{1}$ Centre of Excellence for Nutrition, and ${ }^{2}$ Africa Unit for Transdisciplinary Health Research, North-West University, Potchefstroom, South Africa; ${ }^{3}$ University Medical Center, Utrecht, The Netherlands; ${ }^{4}$ Massey University, Auckland, New Zealand 